\title{
Leistungsorientierte Entlohnung in der Landwirtschaft: eine empirische Analyse
}

\author{
Dissertation \\ zur Erlangung des Doktorgrades \\ der Fakultät für Agrarwissenschaften \\ der Georg-August-Universität Göttingen
}

vorgelegt von

Juliane Zazie von Davier geboren in Berlin

Göttingen, im Juli 2007 
D 7

1. Referentin/Referent: Prof. Dr. Enno Bahrs

2. Korreferentin/Korreferent: Prof. Dr. Ludwig Theuvsen

Tag der mündlichen Prüfung: 19.07.2007 


\section{Danksagung}

Wie bei den meisten Dissertationen ist die Fertigstellung der Arbeit der Unterstützung vieler Menschen $\mathrm{zu}$ verdanken. Zunächst möchte ich mich auf diesem Wege ganz herzlich bei meinem Doktorvater Prof. Dr. Enno Bahrs für die Überlassung des bis zuletzt spannenden Themas und die Betreuung bedanken, die stets von Kontinuität aber auch von den nötigen Freiräumen geprägt war. Der Landwirtschaftlichen Rentenbank und der Landwirtschaftskammer Niedersachsen gilt mein Dank für die Unterstützung der Befragungen. Die vielen landwirtschaftlichen Betriebsleiter und Mitarbeiter, die sich die Mühe gemacht haben, den Fragebogen auszufüllen, sind die zentralen Stützen dieser Arbeit.

Prof. Dr. Ludwig Theuvsen danke ich für die Übernahme des Korreferats und die große fachliche und persönliche Unterstützung. Prof. Dr. Reiner Doluschitz und Prof. Dr. Achim Spiller verdanke ich hilfreiche Anmerkungen zu meiner Arbeit im Rahmen der Doktorandenseminare.

Meine Kollegen im Blauen Turm haben die letzten Jahre zu einer unvergesslichen Zeit gemacht, hierzu hat vor allem Nils Banse beigetragen. Diese bedeutende Phase meines Lebens wird untrennbar mit der Erinnerung an ihn verbunden sein.

Insa Rust, Sabine Gerlach, Jobst-Hendrik-Held und Christoph Bollmann waren emsige Korrekturleser und gute Gesprächspartner in jeder Lebenslage. Mein Zimmernachbar Thorsten Hollmann-Hespos hat manches Word-Problem behoben und mir viele heitere Stunden beschert.

Meinen zahlreichen Babysittern ist der Abschluss der Dissertation zu verdanken. Oliver Ebneth, Bianka Rieck, Monika Scholz, Martina Reichmann, Tuba Pekkirbizli, Bianca Hesse sowie Eva, Bianca, Helene, Mauritz und Julius v. Davier haben Prüfungsbeisitz, Vortragsreisen oder einfach nur entspanntes Mittagessen ermöglicht.

Für Philipp und Fridolin bedeutete die Promotion oft Verzicht auf gemeinsame, entspannte Stunden. Ihre Geduld und Liebe ist unermesslich, ihnen widme ich diese Arbeit. 
Inhaltsverzeichnis I

Tabellenverzeichnis $\quad$ V

$\begin{array}{ll}\text { Abbildungsverzeichnis } & \text { VII }\end{array}$

$\begin{array}{lll}\text { Abkürzungsverzeichnis } & \text { VIII }\end{array}$

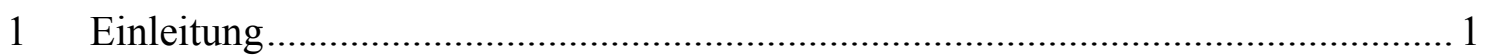

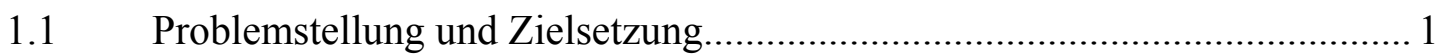

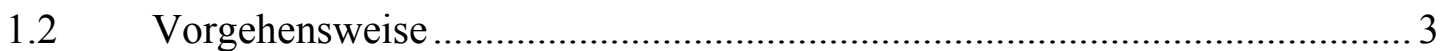

1.3 Abgrenzung des Untersuchungsgegenstandes ............................................ 5

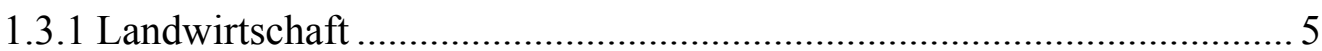

1.3.2 Leistungsorientierte Entlohnung als materielles Anreizsystem............... 6

2 Rahmenbedingungen der Leistungsentlohnung in der Landwirtschaft .................... 8

2.1 Zu den Besonderheiten der Landwirtschaft .............................................. 8

2.2 Zur Beschäftigungssituation in der Landwirtschaft in Deutschland............. 13

2.2.1 Bestimmungsgründe der Beschäftigung in der Landwirtschaft.............. 13

2.2.2 Die statistisch erfasste Beschäftigungssituation in der deutschen Landwirtschaft

2.2.2.1 Methodische Vorbemerkungen zu den Veränderungen in der Arbeitskräfteerhebung.....

2.2.2.2 Die statistisch erfasste Beschäftigungssituation in den landwirtschaftlichen Betrieben des früheren Bundesgebiets ...... 20

2.2.2.3 Die statistisch erfasste Beschäftigungssituation in den landwirtschaftlichen Betrieben der neuen Bundesländer

2.2.2.4 Die statistisch erfasste Beschäftigungssituation in den landwirtschaftlichen Betrieben Deutschlands

2.2.3 Prognosen zum Fachkräftebedarf in der Landwirtschaft....................... 30

2.3 Arbeitsrechtliche Rahmenbedingungen ................................................... 35

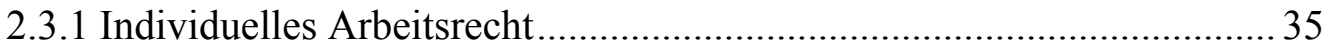

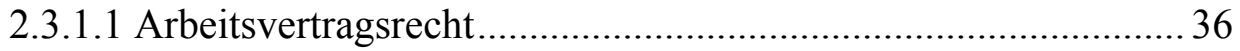

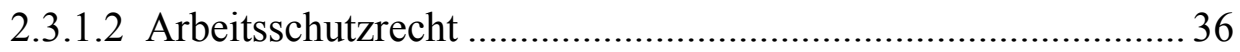

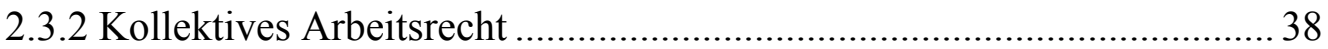

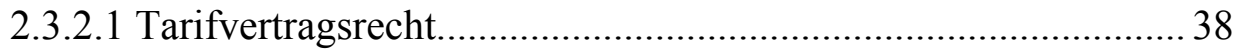

2.3.2.2 Betriebsverfassungsrecht und Mitbestimmungsrechte der Arbeitnehmer 40

3 Leistungsorientierte Entlohnung im theoretischen Kontext ............................... 43

3.1 Institutionen- und personalökonomische Forschungsansätze ....................... 44 


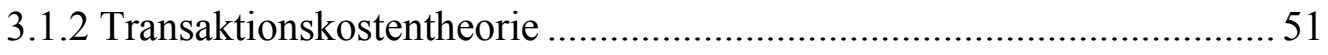

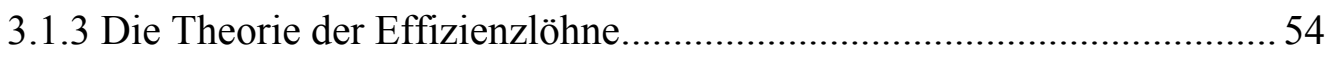

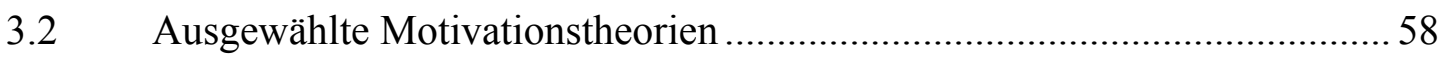

3.2.1 Bedürfnishierarchie nach Maslow ...................................................... 58

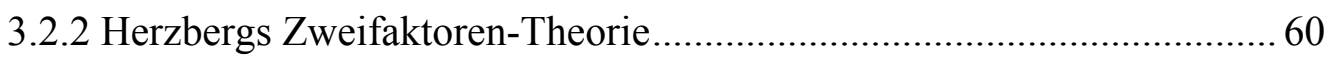

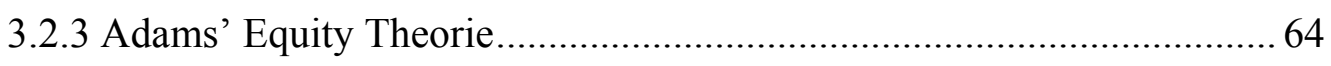

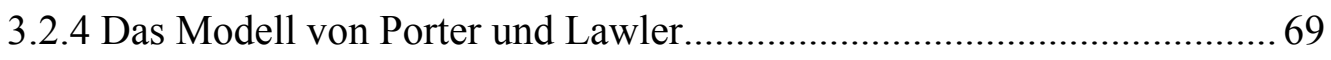

3.3 Experimentelle Ökonomie und Mitarbeitermotivation................................ 72

3.3.1 Besitzeffekt, Verlustaversion und Status Quo Bias .............................. 73

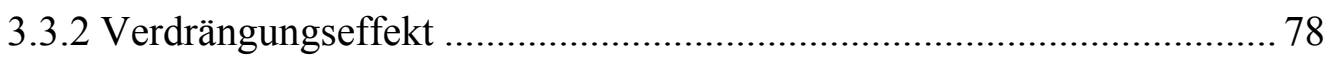

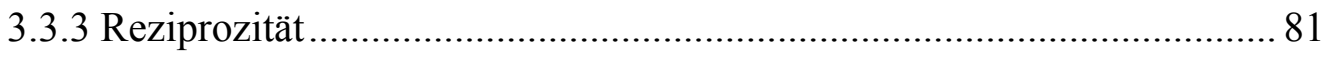

3.3.4 Experimente zur Bedeutung von Fairness ......................................... 84

3.4 Erfolgsbedingungen leistungsorientierter Lohngestaltung .......................... 86

4 Gestaltungsmöglichkeiten materieller Anreizsysteme........................................ 89

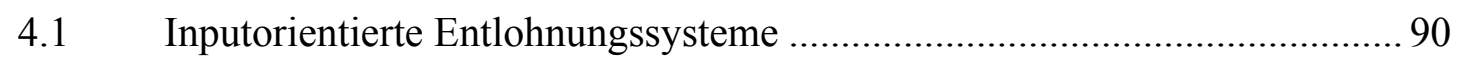

4.1.1 Anforderungsabhängige Löhne........................................................ 90

4.1.2 Sozialstatus- und lebensabschnittsabhängige Löhne ............................ 93

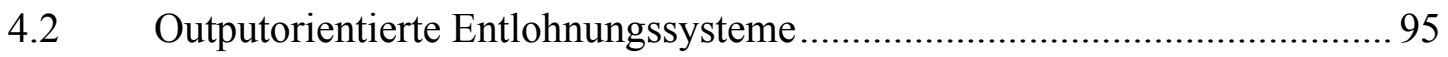

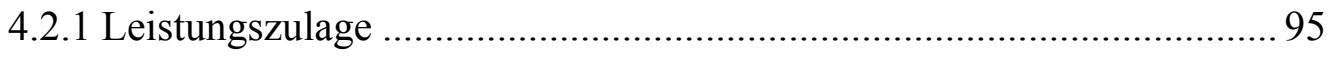

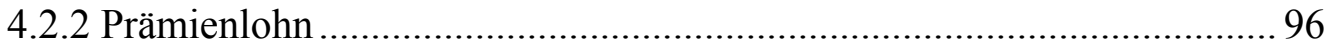

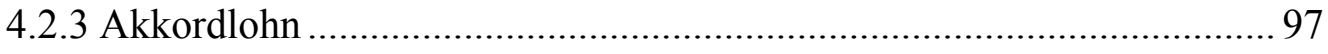

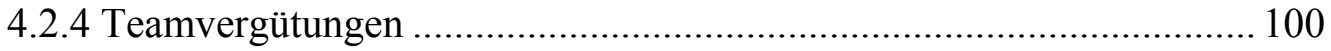

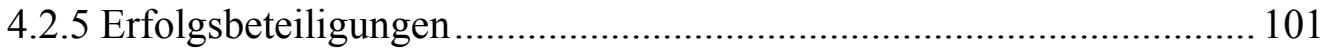

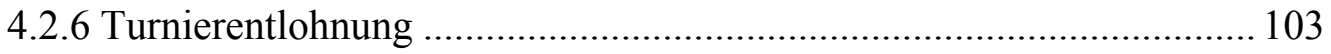

4.3 Cafeteria-Systeme als individualisierte Vergütungsform .......................... 107

5 Empirische Analyse zur leistungsorientierten Entlohnung in der Landwirtschaft 110

5.1 Methodik und Durchführung der Befragungen ........................................ 110

5.1.1 Arbeitshypothesen und Untersuchungsmodell der Befragungen.......... 112

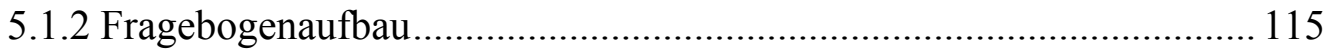

5.1.3 Datenerhebung und -auswertung der Betriebsleiterbefragung............. 117

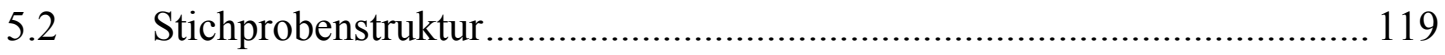

5.2.1 Stichprobenstruktur der Arbeitgeberbefragung ................................. 119

5.2.2 Stichprobenstruktur der Arbeitnehmerbefragung .............................. 123

5.3 Ergebnisse zu den Erfolgsbedingungen leistungsorientierter Entlohnung . 125

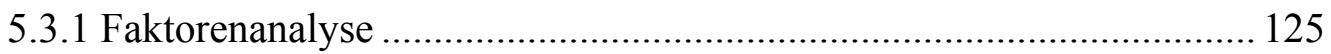


5.3.2 Motivation der Mitarbeiter in den untersuchten Betrieben aus Sicht der

Betriebsleiter

5.3.3 Intrinsische Motivation der befragten Arbeitnehmer.......................... 134

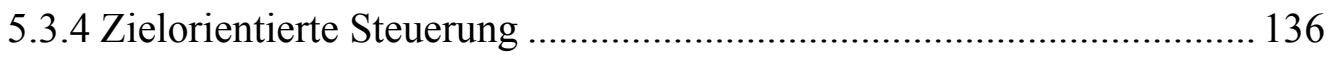

5.3.5 Zum Stellenwert extrinsischer Anreize............................................ 139

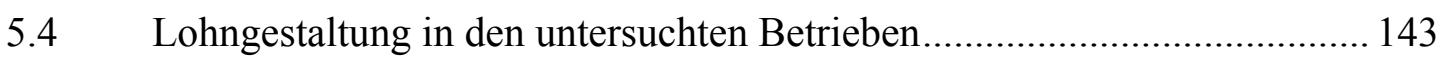

5.4.1 Entlohnungssysteme bei den Mitarbeitern in der Produktion............... 143

5.4.2 Leistungsindikatoren in den analysierten Betrieben .......................... 147

5.4.3 Entlohnungssysteme bei den Führungskräften ................................ 152

5.4.4 Lohnhöhe in den analysierten Betrieben ......................................... 154

5.5 Erfahrungen mit leistungsorientierter Lohngestaltung ............................ 157

5.5.1 Erfahrungen der Betriebsleiter...................................................... 157

5.5.1.1 Ergebnisse der logistischen Regression.................................. 157

5.5.1.2 Clusteranalyse zu den Betriebsleitererfahrungen mit

Leistungslöhnen ............................................................... 163

5.5.2 Erfahrungen der Arbeitnehmer mit leistungsorientierter Entlohnung .. 170

5.6 Zusammenfassung der Ergebnisse hinsichtlich der Arbeitshypothesen ..... 174

6 Handlungsempfehlungen für die landwirtschaftliche Praxis .............................. 177

6.1 Leitfaden zur leistungsorientierten Lohngestaltung ................................ 177

6.1.1 Zur Effektivität extrinsischer Anreize in der Landwirtschaft .............. 179

6.1.2 Möglichkeiten der zielorientierten Steuerung in den Betrieben ........... 182

6.2 Indikatoren für Leistungslöhne in der Landwirtschaft .............................. 183

6.2.1 Leistungsindikatoren für den Ackerbau........................................... 184

6.2.2 Leistungsindikatoren für die Milchproduktion ................................. 193

6.2.3 Leistungsindikatoren für die Sauenhaltung und Schweinemast ........... 199

6.2.3.1 Leistungsindikatoren für die Sauenhaltung ............................. 199

6.2.3.2 Leistungsindikatoren für die Schweinemast ........................... 202

6.2.3.3 Zusammenfassende Beurteilung der Leistungsindikatoren für die

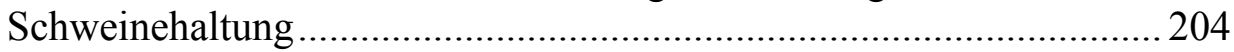

6.2.4 Leistungsindikatoren für die Biogas- und Stromproduktion ............... 205

6.3 Exemplarische betriebswirtschaftliche Auswirkungen von Leistungslöhnen

in verschiedenen Betriebszweigen.... 208

6.3.1 Exemplarische betriebswirtschaftliche Auswirkungen eines

Leistungslohnsystems im Ackerbau

6.3.2 Exemplarische betriebswirtschaftliche Auswirkungen eines

Leistungslohnsystems in der Milchproduktion. 
6.3.3 Exemplarische betriebswirtschaftliche Auswirkungen eines Leistungslohnsystems in der Sauenhaltung ....................................... 214

6.3.4 Exemplarische betriebswirtschaftliche Auswirkungen eines Leistungslohnsystems für Biogasanlagen.... 216

6.4 Perspektiven der leistungsorientierten Entlohnung in der Landwirtschaft ...... 218

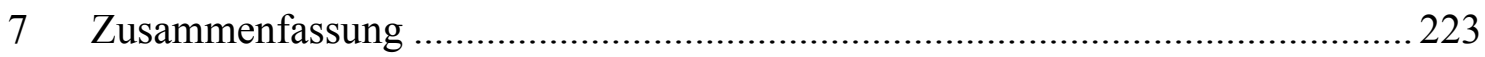

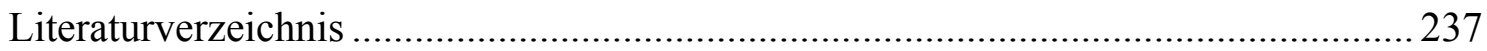




\section{Tabellenverzeichnis}

Tabelle 1: Durchschnittliche Bruttoverdienste und bezahlte Stunden der männlichen Arbeiter in der Landwirtschaft...................................................................... 15

Tabelle 2: Bruttolohnvergleich der in der Landwirtschaft Beschäftigten mit anderen

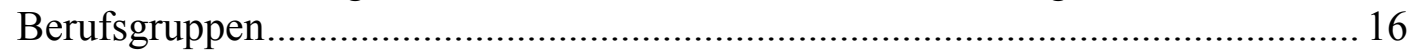

Tabelle 3: Bruttolohnvergleich der in der Landwirtschaft Beschäftigten mit anderen

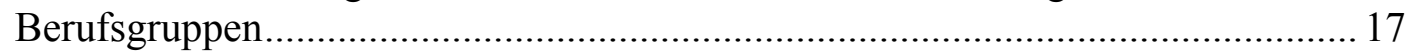

Tabelle 4: Ausgewählte Ergebnisse zur Beschäftigung in den landwirtschaftlichen

Betrieben des früheren Bundesgebiets.............................................................. 21

Tabelle 5: Ausgewählte Ergebnisse zur Beschäftigung in den landwirtschaftlichen

Betrieben in den neuen Ländern ......................................................................... 23

Tabelle 6: Ausgewählte Ergebnisse zur Beschäftigung in den landwirtschaft-lichen

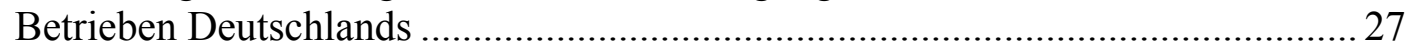

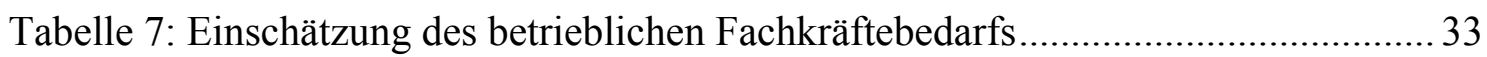

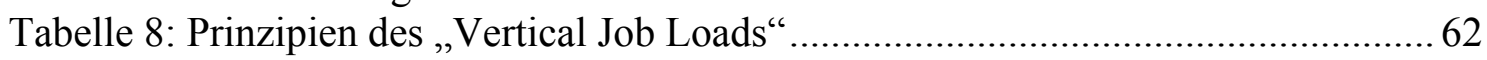

Tabelle 9: Lohngruppenbeschreibung und Stundenlöhne für ständig beschäftigte

Landarbeiter in Schleswig-Holstein................................................................ 91

Tabelle 10: Prämienarten und -indikatoren .............................................................. 96

Tabelle 11: Fragebogenaufbau der Betriebsleiter- und Mitarbeiterbefragung .............. 116

Tabelle 12: Rücklaufquote der Betriebsleiterbefragung, zweite Erhebungsphase ....... 118

Tabelle 13: Durchschnittliche Flächenausstattung und Bestandesgrößen nach

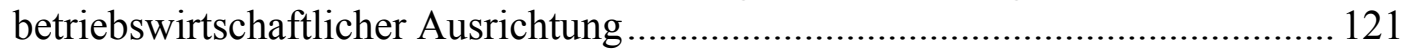

Tabelle 14: Nutzung von Beratungsträgern ................................................................. 123

Tabelle 15: Rotierte Komponentenmatrix zu den Erfolgsbedingungen ....................... 127

Tabelle 16: Korrelationen zwischen der Zufriedenheit mit den Mitarbeitern und

verschiedenen Einflussgrößen ............................................................................. 131

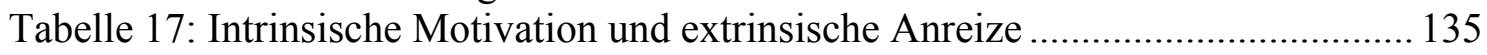

Tabelle 18: Intrinsische Motivation und immaterielle Anreize .................................... 136

Tabelle 19: Anreizsysteme in den untersuchten Betrieben.......................................... 140

Tabelle 20: Entlohnungssysteme bei den Mitarbeitern in der Pflanzen- und

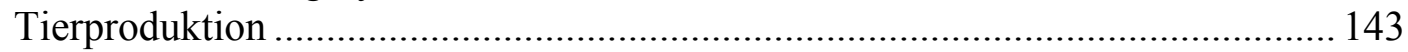

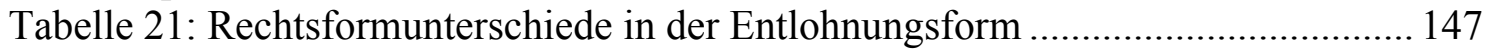

Tabelle 22: Leistungsindikatoren im Ackerbau........................................................... 148

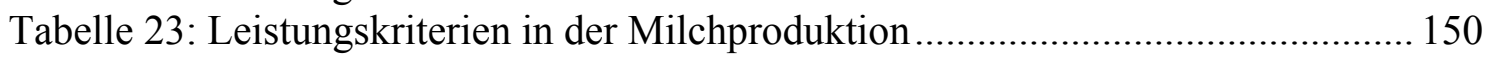

Tabelle 24: Leistungskriterien in der Schweineproduktion ...................................... 151

Tabelle 25 : Entlohnungssysteme bei den Führungskräften ...................................... 152

Tabelle 26: Durchschnittliche Bruttolöhne (Arbeitnehmerbrutto) in den analysierten

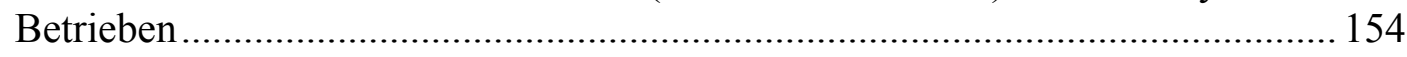

Tabelle 27: Einfluss der Betriebs- und Bestandesgrößen auf die Erfahrung mit

leistungsorientierter Entlohnung..................................................................... 160

Tabelle 28: Binäres Logit-Modell zur Erfahrung mit leistungsorientierter Entlohnung

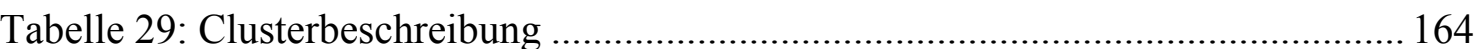

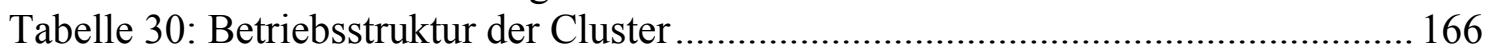

Tabelle 31: Leistungsindikatoren für den Ackerbau nach Arbeitsschritten .................. 185

Tabelle 32: Leistungsindikatoren für den Ackerbau anhand von Kosten-

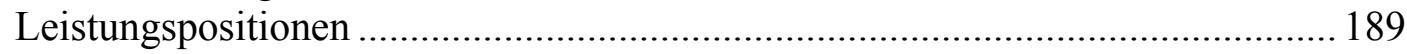

Tabelle 33: Mögliche Leistungsindikatoren in der Milchproduktion.......................... 194

Tabelle 34: Leistungsindikatoren für die Sauenhaltung .......................................... 200 


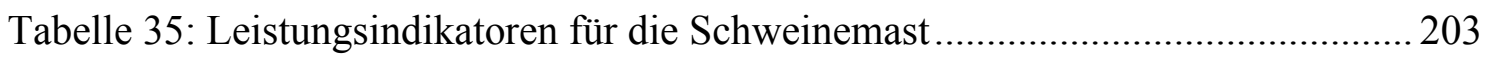

Tabelle 36: Mögliche Leistungsindikatoren in Biogasanlagen.......................................206

Tabelle 37: Kosten und Nutzen des Leistungslohnsystems im Ackerbau ..................... 211

Tabelle 38: Kosten und Nutzen des Leistungslohnsystems in der Milchproduktion ... 213

Tabelle 39: Kosten und Nutzen des Leistungslohnsystems in der Ferkelproduktion... 215

Tabelle 40: Kosten und Nutzen des Leistungslohnsystems in der Biogasproduktion .. 217 


\section{Abbildungsverzeichnis}

Abbildung 1: Vorgehensweise 3

Abbildung 2: Elemente von Anreizsystemen (vereinfacht).

Abbildung 3: Altersstruktur der ständig beschäftigten Lohnarbeitskräfte in der

Landwirtschaft im Jahr 2005

Abbildung 4: Transaktionskosten von Familien- und Lohnarbeitskräften bei gemischt spezifischen Investitionen

Abbildung 5: Das Effizienzlohnmodell von Shapiro und Stiglitz .................................. 55

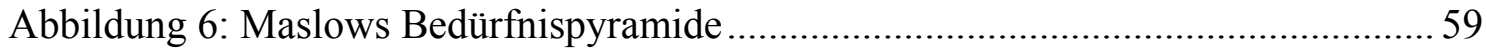

Abbildung 7: Faktoren, die die Haltung zur Arbeit beeinflussen ................................. 61

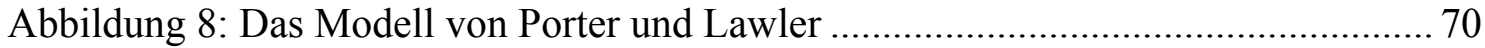

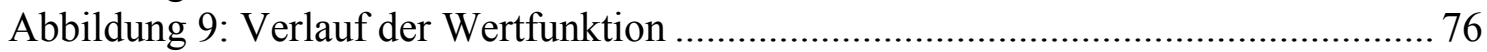

Abbildung 10: Erfolgsbedingungen leistungsorientierter Entlohnung ............................ 86

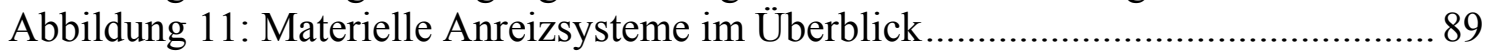

Abbildung 12: Stunden- versus Akkordentlohnung in der Rinderzucht......................... 99

Abbildung 13: Bestimmung der Arbeitsanstrengung bei der Turnierentlohnung ........ 106

Abbildung 14: Untersuchungsmodell der empirischen Analyse .................................. 112

Abbildung 15: Familienfremde Arbeitskräfte in den untersuchten Betrieben.............. 122

Abbildung 16: Höchste Bildungsabschlüsse der befragten Mitarbeiter ....................... 124

Abbildung 17: „Meine Mitarbeiter erledigen ihre Arbeit gewissenhaft und zu meiner vollsten Zufriedenheit"“

Abbildung 18: Anzahl der Lohnarbeitskräfte und Zufriedenheit mit den Mitarbeitern 132

Abbildung 19: Polaritätenprofil zur Motivation und dem Arbeitsverhalten.

Abbildung 20: Einschätzung der Betriebsleiter und Mitarbeiter zur Zielklarheit ........ 137

Abbildung 21: Die fünf wichtigsten Anreizsysteme aus Mitarbeitersicht..................... 140

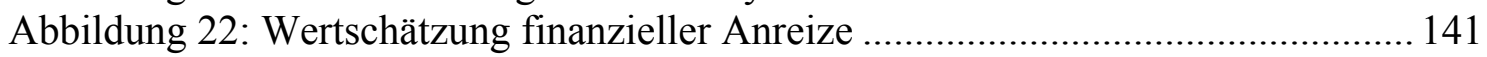

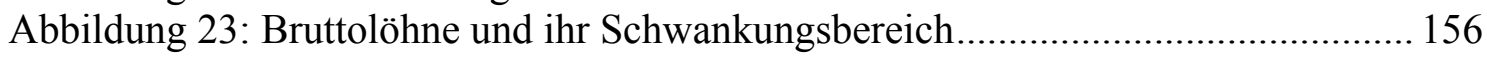

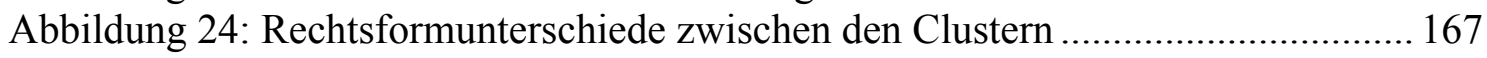

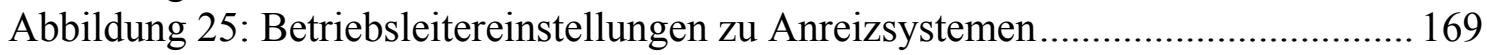

Abbildung 26: Zufriedenheit der Mitarbeiter mit Leistungslöhnen............................... 171

Abbildung 27: „Zulagen, die ich für meine Leistung erhalte, erhöhen meine Motivation“

Abbildung 28: „Ich fühle mich bei der Bezahlung gegenüber meinen Kollegen im Betrieb ungerecht behandelt".....

Abbildung 29: Leitfaden zum Einsatz leistungsorientierter Entlohnung...................... 178 


\section{Abkürzungsverzeichnis}

\begin{tabular}{|c|c|}
\hline $\mathrm{ABL}$ & alte Bundesländer \\
\hline $\mathrm{AG}$ & Aktiengesellschaft \\
\hline $\mathrm{AK}$ & Arbeitskraft \\
\hline AKE & Arbeitskrafteinheit \\
\hline ArbZG & Arbeitszeitgesetz \\
\hline BGB & Bürgerliches Gesetzbuch \\
\hline BGB1. & Bundesgesetzblatt \\
\hline BHKW & Blockheizkraftwerk \\
\hline BMVEL & $\begin{array}{l}\text { Bundesministerium für Verbraucherschutz, Ernährung und } \\
\text { Landwirtschaft }\end{array}$ \\
\hline BetrVG & Betriebsverfassungsgesetz \\
\hline BV & Betriebsvergleich \\
\hline bspw. & beispielsweise \\
\hline bzw. & beziehungsweise \\
\hline ca. & circa \\
\hline d.h. & das heißt \\
\hline DM & Deutsche Mark \\
\hline DLG & Deutsche Landwirtschaftsgesellschaft \\
\hline DrittelbG & Drittelbeteiligungsgesetz \\
\hline DTV & Deutscher Taschenbuchverlag \\
\hline $\mathrm{dt}$ & Dezitonne \\
\hline $\mathrm{ECM}$ & energy corrected milk \\
\hline EEG & Erneuerbare-Energien-Gesetz \\
\hline $\mathrm{eG}$ & eingetragene Genossenschaft \\
\hline EG & Europäische Gemeinschaft \\
\hline ERC & Efficiency, Reciprocity and Competitiveness \\
\hline etc. & et cetera \\
\hline et al. & et alii \\
\hline evtl. & eventuell \\
\hline FAL & Bundesforschungsanstalt für Landwirtschaft \\
\hline F.A.Z. & Frankfurter Allgemeine Zeitung \\
\hline FE & Fett und Eiweiß \\
\hline
\end{tabular}


f.

ff.

folgende

GbR

fortfolgende

ggf.

Gesellschaft bürgerlichen Rechts

$\mathrm{GmbH}$

gegebenenfalls

H.

Gesellschaft mit beschränkter Haftung

ha

Hypothese

Hrsg.

Hektar

IAB

Herausgeber

IG

Institut für Arbeitsmarkt- und Berufsforschung

$\mathrm{kg}$

Industriegewerkschaft

$\mathrm{KG}$

Kilogramm

$\mathrm{KGaA}$

Kommanditgesellschaft

KMO

Kommanditgesellschaft auf Aktien

$\mathrm{kWh}_{\mathrm{el}}$

Kaiser-Meyer-Olkin-Kriterium

KWK

Kilowattstunde elektrisch

NAWARO

Kraftwärmekopplung

NBL

Nachwachsende Rohstoffe

$\mathrm{Nr}$.

Neue Bundesländer

NSC

Nummer

NIMBY

No-Shirking-Constraint

LF

Not in my backyard

LMS

Landwirtschaftliche Nutzfläche

Landwirtschaftsberatung Mecklenburg-Vorpommern / Schleswig-

Holstein

LPG Landwirtschaftliche Produktionsgenossenschaft

LTA Landwirtschaftlich-Technische/r Assistentin/-en

OHG

Offene Handelsgesellschaft

PA

Prinzipal-Agent/ Prinzipal-Agenten

$\mathrm{RSH}$

Rinderzucht Schleswig-Holstein eG

S.

Seite

Sp.

Spalte

SPSS

Statistical Product and Service Solution

$\mathrm{TF}$

technischer Fortschritt

TVG

Tarifvertragsgesetz 
TzBfG

u.a.

usw.

versch

vgl.

WJ

WTA

WTP

QS

z.B.

z.T.
Teilzeit- und Befristungsgesetz

unter anderem

und so weiter

verschiedene

vergleiche

Wirtschaftsjahr

Willingness to accept

Willingness to pay

Qualität und Sicherheit

zum Beispiel

zum Teil 
„, Neben seiner Landwirtschaft, die im Frühjahr besondere Aufmerksamkeit erforderte, und neben seiner Lektüre hatte Lewin schon im Winter begonnen, eine landwirtschaftliche Abhandlung zu schreiben, deren Hauptgedanke war: in der Landwirtschaft müsse man den Charakter des Arbeiters als gegebene Größe auffassen, wie Klima und Bodenbeschaffenheit, und darum müsse die Wissenschaft von der Landwirtschaft nicht von zwei Größen, Klima und Bodenbeschaffenheit, ausgehen, sondern von dreien, Klima, Bodenbeschaffenheit und dem bekannten unveränderlichen Charakter des Arbeiters. "

Lew N. Tolstoj in „Anna Karenina“

\section{$1 \quad$ Einleitung}

\subsection{Problemstellung und Zielsetzung}

Die deutsche Landwirtschaft ist von einem deutlichen Strukturwandel geprägt. Im Rahmen einer zunehmenden Liberalisierung der Märkte wird dieser weiter beschleunigt. Zwar ist der Familienbetrieb im früheren Bundesgebiet nach wie vor die wichtigste Arbeitsverfassung, die Bedeutung von familienfremden Arbeitskräften nimmt jedoch merklich zu. In den neuen Bundesländern zeigt sich eine gegenläufige Tendenz: im Zuge der Umstrukturierung der ostdeutschen Landwirtschaft kam es zu zahlreichen Neu- und Ausgründungen landwirtschaftlicher Familienbetriebe. Aus diesem Grunde erhöht sich hier der Anteil der Familienarbeitskräfte an den in der Landwirtschaft beschäftigten Arbeitskräften. Nach wie vor stellen aber die ständig beschäftigten Fremdarbeitskräfte den wichtigsten Personenkreis in den neuen Bundesländern (StATISTISCHES BundESAMT, versch. Jahrgänge). Die Bedeutung von Fremdarbeitskräften wird auch vor dem Hintergrund sich wandelnder Erwerbsquellen für die Landwirtschaft, hier sind z.B. die regenerativen Energien zu nennen, weiterhin steigen.

Durch die vorherrschende Arbeitsverfassung des Familienbetriebs hatten personalwirtschaftliche Fragestellungen wie die Gestaltung von Anreizsystemen zur Mitarbeitermotivation für die agrarökonomische Forschung in Deutschland bis zur Wiedervereinigung kaum praktische Relevanz. Das Forschungsgebiet Personalmanagement in der Landwirtschaft wurde deshalb wenig bearbeitet. Empirische Erhebungen zum Thema Anreizsysteme in der Landwirtschaft beschränken 
sich auf die neuen Bundesländer und beziehen sich auf z. T. länger zurückliegende Zeiträume (siehe z.B. Doluschitz ET AL. 1996, BECKMANN 2000, WÜLlMERS 1995).

Die vorliegende Arbeit behandelt deshalb den aktuellen Stand der Gestaltung von Anreizsystemen in der Landwirtschaft mit Hilfe einer empirischen Analyse vor dem Hintergrund einzelner maßgeblicher Theorien. Sie konzentriert sich dabei auf die Möglichkeiten und Grenzen leistungsorientierter Entlohnung in der Landwirtschaft.

Zahlreiche wissenschaftliche Beiträge aus der betriebswirtschaftlichen Forschung aber auch Artikel in Tageszeitungen sind dem Thema leistungsabhängige Entlohnung gewidmet. So tituliert die F.A.Z. vom 11. August 2003 einen Artikel zur leistungsabhängigen Entlohnung mit der Überschrift „Ohne Preis kein Fleiß“. Auch in landwirtschaftlichen Fachzeitschriften wird immer häufiger auf die Anpassung der gängigen Entlohnungsformen gedrängt, die zu mindestens auf Ebene der ständig beschäftigten Lohnarbeitskräfte bisher vorwiegend als Zeitlohn erfolgt.

Leistungsorientierte Entlohnung wird als Kennzeichen fortschrittlicher Unternehmensführung und Motor zur Mitarbeitermotivation gesehen (KOSCHWITZ UND DIPPMANN 1997: 18 ff.). Während in der betriebswirtschaftlichen Forschung die Einführung von Leistungslöhnen mit dem Hinweis auf mögliche negative Auswirkungen auf die Mitarbeitermotivation mitunter kontrovers diskutiert wird, sind zumindest in deutschen landwirtschaftlichen Fachzeitschriften die Hinweise auf negative Effekte von Leistungslöhnen eher rar (HELLER 2003).

Die vorliegende Arbeit liefert einen Beitrag zur Beantwortung der folgenden Fragen:

- Welche Anreizsysteme - mit der Schwerpunktlegung auf den Entlohnungssystemen - finden in der landwirtschaftlichen Praxis in den verschiedenen betriebswirtschaftlichen Ausrichtungen und Rechtsformen Anwendung?

- In welchen betriebswirtschaftlichen Ausrichtungen der Landwirtschaft sind leistungsorientierte Lohngestaltungen sinnvoll?

- Welche Leistungsindikatoren werden in den untersuchten Betrieben angewendet und besteht in diesem Punkt Verbesserungsbedarf vor dem Hintergrund der theoretischen Grundlagen? 
- Welche Erfahrungen haben Arbeitgeber und -nehmer mit leistungsorientierten Entlohnungssystemen gesammelt?

- Welche Perspektiven hat die leistungsorientierte Lohngestaltung in der Landwirtschaft - auch vor dem Hintergrund wachsender Betriebsgrößen und dem technischen Fortschritt?

Durch die Beantwortung der aufgeführten Fragen sollen Handlungsempfehlungen für die landwirtschaftliche Praxis zur leistungsorientierten Lohngestaltung abgeleitet werden.

\subsection{Vorgehensweise}

Abbildung 1 zeigt die Vorgehensweise und die durchgeführten Arbeitsschritte der vorliegenden Arbeit.

\section{ABBILDUNG 1: VORGEHENSWEISE}

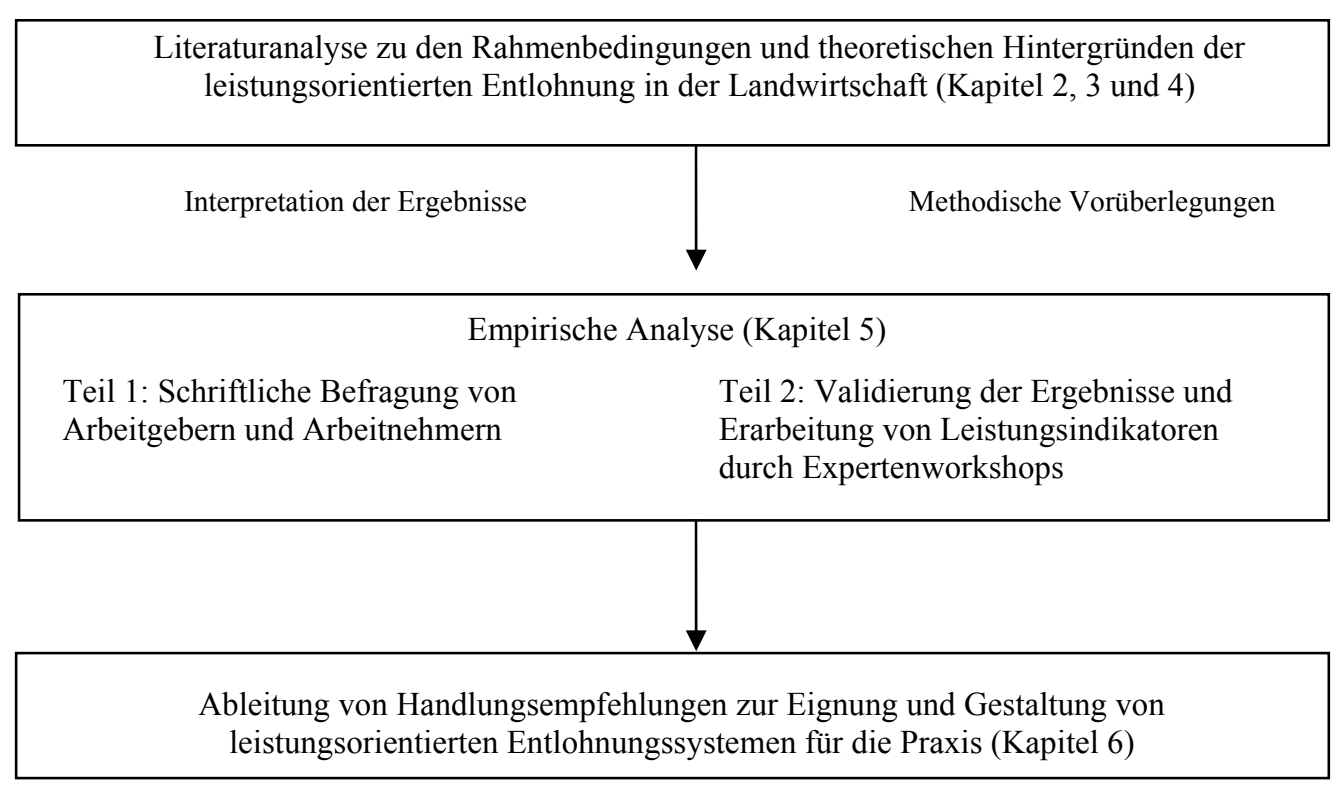

Quelle: Eigene Darstellung

Im ersten Teil (Kapitel 2) werden ausgewählte Rahmenbedingungen der leistungsorientierten Entlohnung in der Landwirtschaft dargestellt. Für die Gestaltung von Anreiz- und Entlohnungssystemen in der Landwirtschaft haben die natürlichen Rahmenbedingungen eine besonders hohe Bedeutung. Diese werden im Kapitel „Besonderheiten der landwirtschaftlichen Produktion“ eingehend behandelt. 
Als weiterer Schritt wird die Beschäftigungssituation in der Landwirtschaft analysiert. Sie stellt eine wichtige wirtschaftliche Rahmenbedingung für das Personalmanagement in der Landwirtschaft dar. Die Bedeutung der Lohnarbeitskräfte für die Beschäftigung in den landwirtschaftlichen Betrieben Deutschlands soll anhand statistischer Daten herausgearbeitet werden. Außerdem wird eine Abschätzung des zukünftigen Fachkräftebedarfs in der Landwirtschaft vorgenommen, die auf einer Prognose von Betriebsleitern landwirtschaftlicher Unternehmen beruht. Von den politischen bzw. rechtlichen Rahmenbedingungen sind die arbeitsrechtlichen Rahmenbedingungen von besonderer Relevanz. Die Gestaltung von Entgeltsystemen unterliegt diversen arbeitrechtlichen Bestimmungen in deren Grenzen sich der Unternehmer bewegen muss.

Das dritte Kapitel ist der Erarbeitung ausgewählter theoretischer Konzepte zur Gestaltung von Anreizsystemen und hier im Besonderen leistungsorientierter Entgeltsysteme gewidmet. Dieser Teil basiert auf einer Literaturanalyse. Die Ergebnisse der Literaturauswertung dienen zum einen der Hypothesengenerierung für die empirische Analyse dienen, zum anderen werden die Ergebnisse der empirischen Analyse vor dem erarbeiteten theoretischen Hintergrund diskutiert. Die Arbeit verfolgt einen multiparadigmatischen Ansatz. Hieraus resultiert das Problem, dass die in Kapitel 3 vorgestellten Theorien zum Teil widersprüchliche Schlussfolgerungen zur Gestaltung von Anreizsystemen nahe legen. Da die Arbeit einen explorativen Charakter hat, ist es aber sinnvoll, sich nicht auf eine in sich konsistente Theorie festzulegen, sondern verschiedene Ansätze zu verfolgen, um die theoretische Interpretation der empirischen Ergebnisse offener zu halten. Die Auswahl der Theorien erhebt keinen Anspruch auf Vollständigkeit, sondern es sollen verschiedene theoretische Perspektiven aufgezeigt werden.

Im vierten Kapitel werden verschiedene Möglichkeiten der Gestaltung materieller Anreize dargestellt, um die Ergebnisse der empirischen Analyse besser interpretieren zu können. Auf eine detaillierte Darstellung immaterieller Anreizsysteme - wie z.B. der Arbeitszeitflexibilisierung - wird an dieser Stelle verzichtet, da dies den Rahmen der Arbeit überschreiten würde.

Die empirische Analyse, deren Konzeption und Ergebnisse in Kapitel 5 dargelegt werden, erfolgt in zwei Stufen. Der Schwerpunkt liegt in einer schriftlichen 
Arbeitgeber- und Arbeitnehmerbefragung, die mittels uni-, bi- und multivariater Analysemethoden ausgewertet werden. Die zweite Stufe der empirischen Analyse besteht in einer Validierung der Ergebnisse durch Expertenworkshops, die mit landwirtschaftlichen Beratern, Landwirten und Mitarbeitern durchgeführt wurden.

Diese Phase diente außerdem der Erarbeitung Praxis tauglicher Leistungsindikatoren für leistungsorientierte Entlohnungssysteme, die in Kapitel 6 vorgestellt werden. In diesem Kapitel werden auch Handlungsempfehlungen für die landwirtschaftliche Praxis abgeleitet, die sich auf die Erkenntnisse aus der Literaturauswertung $\mathrm{zu}$ den Rahmenbedingungen und theoretischen Konzepten und der empirischen Analyse stützen. Ziel ist es ebenfalls, in diesem Kapitel Perspektiven der leistungsorientierten Lohngestaltung für die deutsche Landwirtschaft aufzuzeigen.

\subsection{Abgrenzung des Untersuchungsgegenstandes}

\subsubsection{Landwirtschaft}

Die Landwirtschaft ist ein Teil der volkswirtschaftlichen Produktion und durch die enge Bindung der landwirtschaftlich tätigen Erwerbspersonen $\mathrm{zu}$ ihrer Arbeit und des stetigen Produktionsverlaufs gleichzeitig ein Teil des Soziallebens. Im Familienbetrieb wird die Landwirtschaft charakterisiert durch eine enge Verbindung von Haushalt und Familie, die eine sozioökonomische Einheit bilden. Die Landwirtschaft beruht definitionsgemäß auf der wirtschaftlichen Nutzung der von Grund und Boden zum Anbau von Nahrungs- und Futtermitteln oder zur Weiterverarbeitung durch Tierzucht oder landwirtschaftliche Nebenbetriebe, zu denen auch Molkereien und Brennereien zählen. Nach REISCH (1993: 2530) gehören auch der Gartenbau und die Fischerei im weitesten Sinne zum Sektor Landwirtschaft. Der Waldbau wird aufgrund seiner besonderen produktionstechnischen und betriebsstrukturellen Eigenheiten gesondert unter dem Begriff Forstwirtschaft geführt.

Aufgrund der heterogenen und speziellen Produktionsverfahren in der Landwirtschaft, deren Besonderheiten bei der Gestaltung von leistungsorientierten Entlohnungssystemen zu beachten sind, beschränkt sich diese Arbeit in diesem Punkt auf einige Produktionsverfahren bzw. betriebswirtschaftliche Ausrichtungen. Dies sind der Ackerbau, ohne Sonder- und Dauerkulturen, die Milchviehhaltung und die Schweineproduktion (Schweinemast und Sauenhaltung). Perspektivisch wird außerdem 
die leistungsorientierte Entlohnung in Biogasanlagen angeschnitten. Nichts desto trotz wären der Obstanbau und die Sonderkulturen ein interessantes Forschungsgebiet, gerade aufgrund der möglichen Akkordfähigkeit der Verfahren (vgl. Kapitel 4).

\subsubsection{Leistungsorientierte Entlohnung als materielles Anreizsystem}

Im weitesten Sinne zählen alle verhaltensbeeinflussenden Stimuli im Betrieb, seien sie unbewusst oder bewusst ausgelöst, zu den Anreizsystemen. Anreizsysteme im engeren Sinne dienen der Erreichung betrieblicher Ziele. Mit Hilfe von Anreizsystemen wird vorrangig versucht, Mitarbeiter $\mathrm{zu}$ motivieren, ein im Sinne des Unternehmens zielgerichtetes Verhalten zu zeigen (BECKER 1995).

Anreizsysteme können verschiedene Funktionen haben. Sie können Eintritts-, Bleibeund Leistungsanreize bieten. Der Fokus dieser Arbeit soll auf den Leistungsanreizen liegen.

Anreizsysteme können unterschieden werden in materielle Anreizsysteme, die auch als Entlohnungs- oder Entgeltsysteme bezeichnet werden, und immaterielle Anreizsysteme (siehe Abbildung 2). Vereinfacht dargestellt ist eine weitere Unterteilung von Entlohnungssystemen in den obligatorischen Zeitlohn bzw. festes Gehalt mit den dazugehörigen Sozialleistungen sowie dem fakultativen und variablen Leistungslohn und Erfolgs- und Kapitalbeteiligungen möglich. ${ }^{1} \mathrm{Zu}$ den immateriellen Anreizsystemen gehören zum Beispiel die Sicherheit des Arbeitsplatzes, die Arbeitsplatzgestaltung, Aus- und Weiterbildungsmöglichkeiten, Karriereaussichten, Arbeitsinhalte, Information und Kommunikation, die Arbeitszeitgestaltung sowie die Organisationskultur.

\footnotetext{
${ }^{1}$ Eine ausführliche Beschreibung der verschiedenen Entlohnungssysteme und ihrer Mischformen findet sich in Kapitel 4.
} 
ABBILDUNG 2: ELEMENTE VON ANREIZSYSTEMEN (VEREINFACHT)

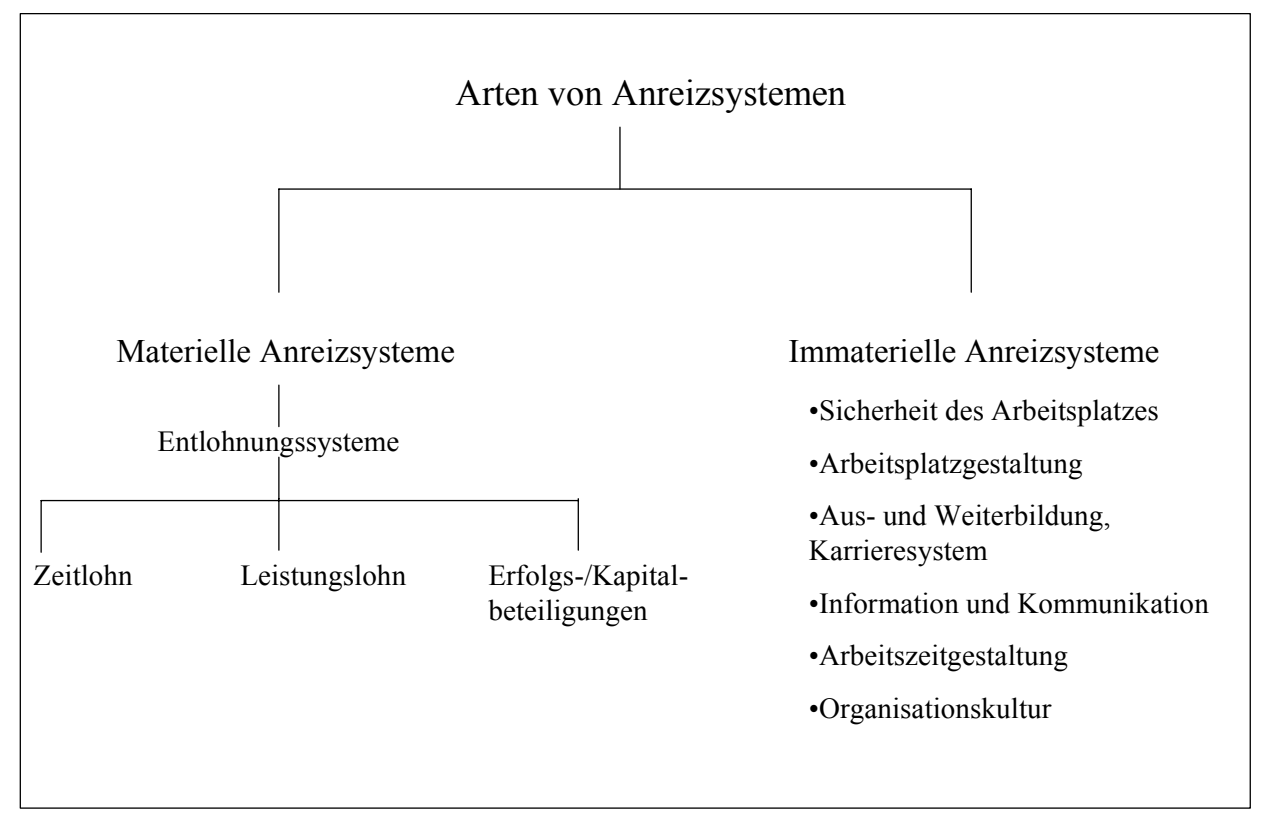

Quelle: Eigene Darstellung in Anlehnung an BECKER 1995

Aufgrund der umfangreichen Gestaltungsmöglichkeiten von Anreizsystemen sollen in dieser Arbeit vor allem die Möglichkeiten und Grenzen materieller Anreizsysteme behandelt werden. Um die Bedeutung materieller Anreize für die Mitarbeitermotivation einordnen zu können, beziehen sich die theoretischen Ausführungen und empirischen Ergebnisse an einigen Stellen auch auf immaterielle Anreize. 


\section{Rahmenbedingungen der Leistungsentlohnung in der Landwirtschaft}

Im folgenden Kapitel werden die Rahmenbedingungen der Gestaltung von Anreizsystemen - mit dem Fokus auf leistungsabhängigen Vergütungsformen - in der Landwirtschaft dargestellt. Hierzu zählen unter anderem natürliche, wirtschaftliche und rechtliche Beschränkungen, denen der landwirtschaftliche Unternehmer in Deutschland unterliegt.

Zunächst sollen einige wichtige Besonderheiten der landwirtschaftlichen Produktion aufgezeigt werden, die diese möglicherweise von anderen Wirtschaftszweigen unterscheidet und die Berechtigung zeigen soll, dieses Thema gesondert für die Landwirtschaft zu behandeln. Im Anschluss daran wird die Bedeutung von Lohnarbeitskräften in der deutschen Landwirtschaft mit Hilfe der Agrarstatistik dargelegt und Prognosen zur zukünftigen Bedeutung familienfremder Fachkräfte in den landwirtschaftlichen Betrieben basierend auf Literaturauswertungen und eigenen Einschätzungen angestellt. Im letzten Teil dieses Kapitels werden dann wichtige rechtliche Rahmenbedingungen für die Gestaltung von Anreizsystemen dargestellt, die die Grenzen der leistungsorientierten Entgeltgestaltung beeinflussen.

\subsection{Zu den Besonderheiten der Landwirtschaft}

Die landwirtschaftliche Produktion weist gegenüber anderen Industrien einige Spezifika auf, aus denen sich verschiedene ökonomische Implikationen ableiten lassen.

\section{Abhängigkeit von der Natur}

Die landwirtschaftliche Produktion unterscheidet sich von anderen Unternehmen durch ihre Abhängigkeit von biologischen Prozessen. Produktionsprozesse können deshalb vom Menschen nicht beliebig manipuliert werden (BRANDES ET AL. 1997: 78, LANGBEHN 1994: 381). Der Ertrag der landwirtschaftlichen Produktion ist im Wesentlichen von den natürlichen Standortbedingungen abhängig und variiert zwischen den Jahren. Mit der Abhängigkeit der landwirtschaftlichen Produktion von biologischen Prozessen und wechselnden klimatischen Bedingungen treten stochastische Größen in den landwirtschaftlichen Prozess ein. Dies erschwert die unmittelbare Kontrolle von Mitarbeiterleistungen, seien sie quantitativer oder qualitativer Art, im Vergleich zu automatisierten Vorgängen. Produktionsökonomische Modelle treffen häufig die 
Annahme, dass der Unternehmer die Produktionsfunktion der von ihm erzeugten Güter mit Sicherheit bestimmen kann (DEBERTIN 1986: 7). Risiko und Unsicherheit sind jedoch ein wesentlicher Bestandteil der landwirtschaftlichen Produktion. Eine weitere Besonderheit der landwirtschaftlichen Produktion ist der Zeitabstand zwischen Input und Output (REISCH 1993: 2530). Dieser „time-lag“ führt dazu, dass UrsacheWirkungs-Beziehungen schwierig zu bestimmen sind.

\section{Bodengebundenheit der landwirtschaftlichen Produktion}

Der Boden nimmt als Produktionsfaktor im landwirtschaftlichen Betrieb eine Sonderrolle ein. Während der Boden in Industrieunternehmen lediglich ein Standortfaktor ist, begrenzt er im landwirtschaftlichen Betrieb, mit Ausnahme einiger Boden ungebundener Betriebszweige der Tierproduktion, das betriebliche Wachstum. Die Bodengebundenheit der landwirtschaftlichen Produktion führt dazu, dass räumliche Konzentrationsprozesse (vor allem in der Pflanzenproduktion) nur begrenzt möglich sind (BRANDES ET AL. 1997: 79, REMMINGHORST 2000: 12, BECKMANN 2000: 157 f.). Die physische Immobilität des Bodens führt dazu, dass die landwirtschaftliche Produktion teilweise mit hohen Transportkosten verbunden ist. Dies trifft im besonderen Maße die Tierproduktion, denn diese ist zwar räumlich stärker konzentrierbar, da bodenunabhängiger, bei Konzentrationsprozessen fallen dann allerdings hohe Transportkosten für Futtermittel und Mist bzw. Gülle an. Strenger werdende Umweltauflagen führen dazu, dass der Konzentration der Tierproduktion auch von institutioneller Seite stärkere Grenzen gesetzt werden.

Boden ist eine heterogene Ressource und sollte nachhaltig genutzt werden, um zum einen negative externe Effekte der landwirtschaftlichen Produktion (z.B. Erosion, Nitratauswaschung) $\mathrm{zu}$ vermeiden und zum anderen die Existenzgrundlage des Betriebes $\mathrm{zu}$ erhalten. Dies ist bei der Gestaltung von Entlohnungssystemen zu berücksichtigen. Ein Mitarbeiter könnte durch das Anreizsystem motiviert sein, den Ertrag, falls dieser die Outputgröße ist an der sich das Entgelt bemisst, zu maximieren. Dies könnte zum Beispiel im Bereich der Pflanzenproduktion zu übersteigertem Einsatz von Produktionsmitteln führen. Ein Mitarbeiter könnte auch kurzfristig den Einsatz von Grunddüngern einsparen und somit langfristig die natürlichen Reserven des Bodens angreifen. 


\section{Flexibilitätserfordernisse im Arbeitsprozess}

Die Abhängigkeit von klimatischen und biologischen Rahmenbedingungen und die Bodengebundenheit der Produktion führen zu Arbeitsprozessen, die durch eine saisonal geprägte Arbeitsbelastung geprägt sind. Besonders die Pflanzenproduktion zeigt zyklische Arbeitssenken und -spitzen, die für die Mitarbeiter in der Landwirtschaft punktuell erhebliche Mehrarbeitsbelastungen mit sich bringen. Aber auch in der Tierproduktion kann es zu einer zeitlich akkumulierten Arbeitsbelastung kommen, z.B. bei saisonal konzentrierten Abkalbungen in der Mutterkuhhaltung oder bei Ablammungen. Die Flexibilitätserfordernisse führen dazu, dass selbst in spezialisierten Betrieben Mitarbeiter häufig in verschiedenen Arbeitsbereichen eingesetzt werden. Auch greifen unterschiedliche Mitarbeiter in den Produktionsprozess ein, so dass der individuelle Leistungsbeitrag schwer zu quantifizieren ist.

\section{Arbeitsethos und Flow-Erlebnis in der Landwirtschaft}

Die zeitweise überdurchschnittliche Arbeitsbelastung und die trotz des arbeitserleichternden technischen Fortschritts physisch anspruchsvollen Tätigkeiten in der Landwirtschaft führen jedoch nicht unbedingt dazu, dass die Arbeit in der Landwirtschaft durchweg negativ besetzt ist. So weist eine Studie des Bielefelder Emnid-Instituts aus dem Jahr 2000 (zitiert in KROMKA 2004: 81 f.) darauf hin, dass $64 \%$ der befragten Landwirte mit ihrem Beruf sehr zufrieden sind, während es bei Nichtlandwirten nur $34 \%$ waren.

KROMKA begründet dieses Ergebnis mit dem Empfinden eines Flow-Erlebnis, das durch die Eigenschaften der landwirtschaftlichen Tätigkeiten begründet ist. Ein Mensch der sich im Flow befindet, empfindet seine Tätigkeit als einen gleichmäßigen Fluss von aufeinander folgenden Handlungsschritten, die ihm ein Gefühl der Selbstbestimmtheit, Zeitlosigkeit und Einheit mit der Umwelt vermitteln (ebenda: 82 f.). Tätigkeiten, die Menschen mit Flow-Erlebnissen verbinden, zeichnen sich durch folgende Eigenschaften aus (ebenda: $83 \mathrm{ff}$ ):

- klare Ziele und ummittelbares Feedback,

- Gleichgewichtigkeit von Fähigkeiten und Anforderungen,

- Aufmerksamkeitssteigerung und Konzentrationszunahme, 
- Gefühl absoluter Kontrolle und Selbstvergessenheit,

- verändertes Zeitgefühl und autotelische Erfahrung.

Die oben genannten Eigenschaften treffen auf viele Tätigkeiten in der Landwirtschaft, unabhängig von der Hierarchieebene zu. Auch die eher einfachen, wiederholt anfallenden Arbeiten wie Feldarbeiten oder Melken bieten den Vorteil, dass das Arbeitsziel klar vor Augen ist und die Arbeit in sich ein direktes Feedback durch das sichtbar Geleistete bietet.

KROMKA stellt in seinen Ausführungen auch die Frage, ob es sich beim Arbeitsethos und der -leidenschaft von Landwirten um eine ,,angeborene Eigenschaft“" handele, oder ob sie nicht vor allem durch die intrinsische Belohnung durch die Tätigkeit an sich begründet sind (ebenda: 91-92).

\section{Die Verflechtung von Familie und Betrieb}

Die Mehrzahl der landwirtschaftlichen Betriebe in Deutschland aber auch in anderen Ländern wird als Familienbetrieb bewirtschaftet.

Der Familienbetrieb hat im Vergleich zu anderen Arbeitsverfassungen Vor- aber auch Nachteile. Zunächst kann davon ausgegangen werden, dass innerhalb der Familie ein gewisser Grad an Altruismus und Loyalität gegenüber der Gemeinschaftsaufgabe herrscht (PollaK 1985: 585 ff., Ait ABDELMALEK 2000: 41), der Motivationsprobleme und Opportunismus vernachlässigbar macht. Gerade für Aufgaben, bei denen der Arbeitseinsatz schwer zu kontrollieren ist, hat der Familienbetrieb einen Vorteil, da er mit weniger Transaktionskosten belastet ist. Auch stehen kurzfristige Ziele gegenüber langfristigen zurück.

Sanktionen für Familienmitglieder, die ihrer Arbeit nicht gerecht werden, sind jedoch nach außen kaum sichtbar. D.h., es ist die Frage, inwieweit die Annahme des Altruismus immer gegeben ist. Dieser hängt auch von der Größe der Familie ab, die mit betrieblichen Arbeiten betraut ist. Die möglichen Stärken des Familienbetriebes, nämlich die überdurchschnittliche intrinsische Motivation und der Arbeitseinsatz der Familienmitglieder, können zugleich seine Schwächen sein. Der Betriebserfolg hängt von eben diesen Erfolgskriterien ab (POLLAK 1985: 587). 
Generationenkonflikte in landwirtschaftlichen Betrieben, die sich nicht zuletzt bei der Hofnachfolge stellen, zeigen, dass die Annahme der unbedingten familiären Harmonie und Loyalität nicht immer zutrifft und auch im Familienbetrieb eigenen Interessen weichen kann.

Einen Betrieb als Familienunternehmen zu führen, heißt auch von den Talenten der Familienmitglieder abhängig zu sein. Je spezifischer und anspruchsvoller die Aufgaben des Unternehmens sind, desto unwahrscheinlicher wird es, dass die Familienmitglieder diese genauso gut oder besser erfüllen können als ein familienfremder, entsprechend ausgebildeter Mitarbeiter (ebenda: 588).

\section{Wirtschaftliche Rahmenbedingungen}

Landwirtschaftliche Unternehmer befinden sich in der Regel in einem polypolistischen Marktumfeld, das sie zu Preisnehmern macht. Die Nachfrage nach Agrarprodukten ist unelastisch und die Einkommenselastizität für Agrarprodukte gering. Fortschreitende Globalisierung der Märkte und ein zunehmender technischer Fortschritt führen dazu, dass die Produzenten sich in einem starken Preisdruck befinden, der jedoch zwischen den Jahren fluktuieren kann (STABLER UND OLFERT 2000: 387). Dieser beeinflusst die Möglichkeiten der Faktorentlohnung. Da im Gewinn maximierenden Unternehmen gemäß neoklassischer Theorie die Faktorentlohnung nach dem Wertgrenzprodukt erfolgt, sind unter anderem die Möglichkeiten einer Lohngestaltung in Anlehnung an die bezahlten Löhne in anderen Wirtschaftszweigen begrenzt (vgl. Kapitel 2.2).

Unterschiedliche wirtschaftliche Standort- und Rahmenbedingungen führen dazu, dass landwirtschaftlich Betriebe sehr unterschiedlich strukturiert, organisiert und ausgestattet sind. Diese werden als integrierende und differenzierende Kräfte bezeichnet und führen dazu, dass sich Betriebe eher spezialisieren oder diversifizieren (DOLUSCHITZ 2001). Innerbetriebliche Verflechtungen führen $\mathrm{zu}$ Wechselwirkungen zwischen den Betriebszweigen, die eine Messbarkeit des Erfolges einzelner Arbeitsschritte noch erschwert. 


\subsection{Zur Beschäftigungssituation in der Landwirtschaft in Deutschland}

Um die Bedeutung des Personalmanagements für die landwirtschaftliche Betriebe in Deutschland abschätzen zu können, ist eine Analyse der Beschäftigungssituation in der Landwirtschaft notwendig. Im landwirtschaftlichen Familienbetrieb mit wenigen oder keinen Lohnarbeitskräften ist das Themenfeld „Personalmanagement“ weniger relevant als in Lohnarbeitsbetrieben. Deshalb soll in diesem Kapitel die Beschäftigungssituation in der deutschen Landwirtschaft dargestellt werden und die wachsende Bedeutung von Lohnarbeitskräften aufgezeigt werden. Außerdem sollen Hinweise auf die potentielle Entwicklung des Arbeitskräftebedarfs gegeben werden. Um die zukünftige Entwicklung des Arbeitskräftebedarfs in der Landwirtschaft abschätzen zu können, werden zunächst die Bestimmungsgründe der Beschäftigung in der Landwirtschaft dargestellt. Anschließend wird die Beschäftigungssituation in der Landwirtschaft Deutschlands anhand von Zeitreihen analysiert. Da sich die Beschäftigungssituation in den landwirtschaftlichen Betrieben der neuen und alten Bundesländer durch die historisch bedingt unterschiedlichen Betriebsstrukturen unterscheidet, wird auf die Gegebenheiten in den beiden Teilen Deutschlands gesondert eingegangen. Im letzten Teil des Kapitels sollen dann Prognosen zur Entwicklung des Arbeitskräftebedarfs in der Landwirtschaft angestellt werden.

\subsubsection{Bestimmungsgründe der Beschäftigung in der Landwirtschaft}

In diesem Kapitel sollen zunächst die Bestimmungsgründe der Beschäftigung in der Landwirtschaft dargelegt werden. Das Ziel dieses Kapitels ist es, die statistischen Zeitreihen zur Beschäftigung besser interpretieren zu können.

Die Entwicklung der Beschäftigung in der Landwirtschaft wird bestimmt durch interne und externe Faktoren.

Die internen oder auch sektoralen Faktoren sind die Determinanten innerhalb des Sektors, die die Entwicklung der Beschäftigung in den landwirtschaftlichen Betrieben bestimmen. Hierzu zählen z.B. Änderungen der Produktionstechnik und der Agrarpolitik, die Entwicklung der landwirtschaftlichen Einkommen sowie Veränderungen der Alterspyramide der in der Landwirtschaft Beschäftigten. 
$\mathrm{Zu}$ den externen Faktoren gehören u.a. die Veränderung der Konsum- und Verzehrgewohnheiten in der Gesellschaft, die Nachfrage nach Arbeitskräften außerhalb des Sektors, die Entwicklung der Kaufkraft, der soziale Status der Lohnarbeitskräfte sowie Veränderungen in der Institution Familie (BLANC UND PERRIER-CORNET 1999: 8).

Die Entscheidung für oder gegen einen Arbeitsplatz in der Landwirtschaft hängt im Wesentlichen von den alternativen Beschäftigungs- und Einkommensverhältnissen in anderen Sektoren ab. Ein hohes außerlandwirtschaftliches Lohnniveau und geringe Arbeitslosigkeit führen zu höheren Opportunitätskosten der landwirtschaftlichen Arbeitskräfte.

Im Folgenden soll kurz auf eine wichtige Determinante der Beschäftigung in der Landwirtschaft eingegangen werden: Die landwirtschaftlichen Löhne und Gehälter im Vergleich zu anderen Branchen.

Tabelle 1 zeigt die durchschnittlichen Bruttoverdienste der in der Landwirtschaft beschäftigten männlichen Arbeiter in Abhängigkeit vom Qualifikationsniveau von 1995 bis 2003 für das frühere Bundesgebiet und die neuen Bundesländer. 
TABELLE 1: DURCHSCHNITTLICHE BRUTTOVERDIENSTE UND BEZAHLTE STUNDEN DER MÄNNLICHEN ARBEITER IN DER LANDWIRTSCHAFT

\begin{tabular}{|c|c|c|c|c|c|}
\hline Arbeitergruppe $\mathrm{e}^{1)}$ & 1995 & 1997 & 1999 & 2001 & 2003 \\
\hline \multicolumn{6}{|l|}{ Früheres Bundesgebiet $^{2)}$} \\
\hline \multicolumn{6}{|c|}{ Stundenverdienst in $€$} \\
\hline Qualifizierte Arbeiter & 9,90 & 10,60 & 10,68 & 11,12 & 11,34 \\
\hline Landarbeiter & 8,88 & 9,62 & 10,16 & 10,70 & 10,84 \\
\hline Nichtqualifizierte Arbeiter & 8,60 & 9,13 & 9,20 & 10,40 & 10,78 \\
\hline Insgesamt & 9,24 & 9,97 & 10,23 & 10,88 & 11,15 \\
\hline \multicolumn{6}{|c|}{ Bezahlte Stunden je Monat } \\
\hline Qualifizierte Arbeiter & 193,8 & 198,2 & 200,6 & 194,7 & 203,7 \\
\hline Landarbeiter & 200,0 & 203,8 & 215,2 & 189,3 & 195,6 \\
\hline Nichtqualifizierte Arbeiter & 195,8 & 191,8 & 184,3 & 175,1 & 190,4 \\
\hline Insgesamt & 196,2 & 198,2 & 201,7 & 189,2 & 193,3 \\
\hline \multicolumn{6}{|l|}{ Neue Länder ${ }^{3)}$} \\
\hline \multicolumn{6}{|c|}{ Stundenverdienst in $€$} \\
\hline Qualifizierte Arbeiter & 6,63 & 6,88 & 7,14 & 7,55 & 7,34 \\
\hline Landarbeiter & 6,71 & 6,94 & 7,18 & 7,41 & 7,11 \\
\hline Nichtqualifizierte Arbeiter & 5,60 & 5,66 & 6,37 & 6,68 & 6,40 \\
\hline Insgesamt & 6,59 & 6,86 & 7,13 & 7,51 & 7,27 \\
\hline \multicolumn{6}{|c|}{ Bezahlte Stunden je Monat } \\
\hline Qualifizierte Arbeiter & 215,4 & 209,5 & 211,0 & 198,6 & 202,9 \\
\hline Landarbeiter & 219,2 & 247,7 & 228,2 & 207,6 & 210,5 \\
\hline Nichtqualifizierte Arbeiter & 214,0 & 223,3 & 218,2 & 206,6 & 197,8 \\
\hline Insgesamt & 215,9 & 216,3 & 213,7 & 199,9 & 204,2 \\
\hline $\begin{array}{l}\text { 1) Nur allgemeine Landwirt } \\
\text { enthalten Zulagen und Zusc } \\
\text { jedoch den Wert unentgeltli } \\
\text { West 3) Ohne Berlin-Ost. }\end{array}$ & $\begin{array}{l}\text { alle Betr } \\
\text { sowie die } \\
\text { vährter De }\end{array}$ & $\begin{array}{l}\text { rößenklas } \\
\text { achleistun } \\
\text { te. 2) Ohr }\end{array}$ & $\begin{array}{l}\text { veils } \mathrm{Se} \\
\text { behalter } \\
\text { burg, B }\end{array}$ & $\begin{array}{l}\text { Die Br } \\
\text { nbestand } \\
\text { Saarland }\end{array}$ & $\begin{array}{l}\text { dienste } \\
\text { licht } \\
\text { erlin- }\end{array}$ \\
\hline
\end{tabular}

Quelle: Statistisches BundesAmT, BMVEL, Tabelle 425

Auffällig sind die geringen Einkommensunterschiede zwischen unqualifizierten und qualifizierten Arbeitskräften. Dies trifft vor allem für die durchschnittlichen Bruttoverdienste in den alten Bundesländern zu. Dort beträgt die Stundenlohndifferenz zwischen den nicht qualifizierten und den qualifizierten Arbeitern im Jahr 2003 knapp $0,6 € / \mathrm{h}$. In den neuen Bundesländern beträgt die Spanne circa $0,9 € / \mathrm{h}$. Das Lohngefälle zwischen den alten und neuen Bundesländern für landwirtschaftliche Arbeiter ist beachtlich. Ein qualifizierter Arbeiter verdient im früheren Bundesgebiet durchschnittlich 4 Euro mehr pro Arbeitsstunde als in den neuen Bundesländern.

Betrachtet man die Anzahl der bezahlten Stunden pro Monat, so wird deutlich, dass der Arbeitsplatz Landwirtschaft für die beschäftigten Lohnarbeitskräfte mit einer in zeitlicher Hinsicht stärkeren Arbeitsbelastung einhergeht als die Beschäftigung in 
anderen Branchen. Durchschnittlich ergibt sich für die landwirtschaftlichen Arbeitnehmer im früheren Bundesgebiet im Jahr 2003 eine Arbeitsbelastung von ca. 44,5 Stunden pro Woche. In den neuen Bundesländern dauert die Arbeitswoche durchschnittlich ca. 47 Stunden.

In Tabelle 2 und Tabelle 3 sind die in der Landwirtschaft gezahlten Bruttolöhne ${ }^{2}$ den in anderen Wirtschaftszweigen gezahlten Bruttolöhnen gegenübergestellt.

\section{TABELLE 2: BRUTTOLOHNVERGLEICH DER IN DER LANDWIRTSCHAFT BESCHÄFTIGTEN MIT ANDEREN BERUFSGRUPPEN}

\begin{tabular}{|c|c|c|c|c|}
\hline & Bruttolöhne in $€ / /$ & & & \\
\hline & Landwirtschaft & $\begin{array}{l}\text { Verarbeitendes } \\
\text { Gewerbe }\end{array}$ & $\begin{array}{l}\text { Holzgewerbe (ohne } \\
\text { Herstellung von } \\
\text { Möbeln) }\end{array}$ & $\begin{array}{l}\text { Herstellung von } \\
\text { Metallerzeugnissen }\end{array}$ \\
\hline \multicolumn{5}{|l|}{ Deutschland gesamt } \\
\hline Obere Gehaltsklasse & 9,34 & 17,41 & 14,58 & 16,49 \\
\hline Mittlere Gehaltsklasse & 8,98 & 13,91 & 12,13 & 14,21 \\
\hline Untere Gehaltsklasse & 8,59 & 12,33 & 10,92 & 12,41 \\
\hline \multicolumn{5}{|l|}{ Früheres Bundesgebiet } \\
\hline Obere Gehaltsklasse & 11,34 & 18,03 & 15,02 & 17,14 \\
\hline Mittlere Gehaltsklasse & 10,84 & 14,59 & 12,71 & 14,83 \\
\hline Untere Gehaltsklasse & 10,78 & 12,63 & 11,42 & 12,68 \\
\hline \multicolumn{5}{|l|}{ Neue Bundesländer } \\
\hline Obere Gehaltsklasse & 7,34 & 12,47 & 11,20 & 12,09 \\
\hline Mittlere Gehaltsklasse & 7,11 & 9,96 & 9,77 & 10,46 \\
\hline Untere Gehaltsklasse & 6,40 & 8,99 & 8,80 & 9,10 \\
\hline
\end{tabular}

Quelle: Statistisches Bundesamt 2005, Statistisches Bundesamt (verschiedene Jahrgänge): Fachserie 16, Reihe 2.1, BMVEL Tabelle 425

Es wird deutlich, dass die in der Landwirtschaft Beschäftigten unabhängig vom Qualifikationsniveau die geringsten Stundenlöhne erhalten. Angesichts der gezahlten

2 Durchschnittliche Bruttolöhne für die männlichen Arbeiter in der Landwirtschaft im früheren Bundesgebiet ohne Hamburg, Bremen und das Saarland. Die Bruttolöhne setzen sich zusammen aus den gesamten Beträgen, die vom Arbeitgeber in jeder Lohnanrechnungsperiode gezahlt werden, das ist der tariflich oder frei vereinbarte Lohn einschließlich tariflicher und außertariflicher Leistungs-, Sozial- und sonstiger Zulagen und Zuschläge. Die obere Gehaltsklasse, entspricht der Gehaltsklasse "qualifizierter Arbeiter", d.h. Arbeiter/-innen, die im Allgemeinen eine Berufsausbildung abgeschlossen haben. Die mittlere Gehaltsklasse entspricht der Gehaltsklasse "Landarbeiter". Sie werden meist als Spezialarbeiter, qualifizierte angelernte Arbeiter, angelernte Arbeiter mit besonderen Fähigkeiten oder ähnlich bezeichnet. Für diese Arbeit haben die Arbeiter meist im Rahmen einer mindestens drei Monate dauernden Anlernzeit mit oder ohne Abschlussprüfung die nötigen Fähigkeiten und Kenntnisse erworben. Die untere Gehaltsklasse entspricht Arbeitern, die mit einfachen, als Hilfsarbeiten zu bewertenden Tätigkeiten beschäftigt sind, für die eine fachliche Ausbildung auch nur beschränkter Art nicht erforderlich ist. Sie werden meist als Hilfsarbeiter, ungelernte Arbeiter oder ähnlich bezeichnet. 
Stundenlöhne und des auffällig starken Lohngefälles ist der Arbeitsplatz Landwirtschaft unattraktiv, was sich negativ auf das Angebot an Arbeitskräften für die Landwirtschaft auswirken kann. Jedoch ist zu bedenken, dass regional immobile Menschen nicht immer die alternativen Verdienstmöglichkeiten haben, die in Tabelle 2 und Tabelle 3 dargestellt sind.

In einigen Regionen Deutschlands bieten sich für die Bewohner außerhalb der Landwirtschaft faktisch keine alternativen Beschäftigungsmöglichkeiten. Es ist daher schwierig abzuschätzen, inwieweit Vergleichslöhne geeignet sind, um direkte Schlussfolgerungen auf das Angebot an qualifizierten Lohnarbeitskräften ableiten zu können. Die Forderung von Experten nach Anhebung der Stundenlöhne in der Landwirtschaft, um dem Problem eines möglichen Fachkräftemangels vorzugreifen, kann daher nur in einigen Regionen helfen. In manchen Regionen ist die Anhebung der Stundenlöhne auf das Niveau alternativer Beschäftigungsmöglichkeiten auch nicht durchführbar, da die Landwirtschaft hinsichtlich der Entlohnungsmöglichkeiten nicht mit Großunternehmen, z. B. aus der Metallindustrie, mithalten kann.

TABELLE 3: BRUTTOLOHNVERGLEICH DER IN DER LANDWIRTSCHAFT BESCHÄFTIGTEN MIT ANDEREN BERUFSGRUPPEN

\begin{tabular}{|c|c|c|c|c|}
\hline & \multicolumn{4}{|c|}{ Bruttolöhne in $€ / h$} \\
\hline & Landwirtschaft & Bergbau & $\begin{array}{l}\text { Herstellung von } \\
\text { Kraftwagen und } \\
\text { Fahrzeugteilen }\end{array}$ & Hoch- und Tiefbau \\
\hline \multicolumn{5}{|l|}{ Deutschland gesamt } \\
\hline Obere Gehaltsklasse & 9,34 & 15,18 & 20,86 & 14,81 \\
\hline Mittlere Gehaltsklasse & 8,98 & 13,33 & 17,08 & 13,13 \\
\hline Untere Gehaltsklasse & 8,59 & 12,31 & 15,22 & 12,31 \\
\hline \multicolumn{5}{|l|}{ Früheres Bundesgebiet } \\
\hline Obere Gehaltsklasse & 11,34 & 15,44 & 21,21 & 15,54 \\
\hline Mittlere Gehaltsklasse & 10,84 & 14,11 & 17,48 & 13,91 \\
\hline Untere Gehaltsklasse & 10,78 & 12,56 & 15,40 & 12,64 \\
\hline \multicolumn{5}{|l|}{ Neue Bundesländer } \\
\hline Obere Gehaltsklasse & 7,34 & 13,29 & 14,73 & 11,78 \\
\hline Mittlere Gehaltsklasse & 7,11 & 11,71 & 11,74 & 10,98 \\
\hline Untere Gehaltsklasse & 6,40 & 8,36 & 10,35 & 10,60 \\
\hline
\end{tabular}

Quelle: siehe Tabelle 2 
Die Höhe der Opportunitätskosten wird ferner bestimmt vom Alter und Qualifikationsniveau der in der Landwirtschaft Beschäftigten. Theoretisch bestehen demnach Wechselwirkungen zwischen den volkswirtschaftlichen Rahmenbedingungen und dem Arbeitsmarkt sowie der relativen Vorzüglichkeit eines Arbeitsplatzes in der Landwirtschaft.

ANDERMANN UND SCHMITT (1996) konnten jedoch für die alten Bundesländer zeigen, dass kurzfristig nur geringe Auswirkungen aktueller Bedingungen auf dem Arbeitsmarkt und in der gesamten Volkswirtschaft auf die Beschäftigungsentwicklung in der Landwirtschaft erkennbar werden. Die Verfasser (ebenda: 631) bieten hierfür die folgenden Erklärungsansätze an (die jedoch nicht auf alle Bereiche bzw. Betriebe uneingeschränkt anwendbar sind):

- Die Arbeit in der Landwirtschaft eröffnet für die Beschäftigten auch Wohngelegenheiten auf den Betrieben und schafft dadurch einen Zusatznutzen gegenüber Arbeitsplätzen in anderen Branchen.

- Eine landwirtschaftliche Tätigkeit erfordert sektorspezifische Fertigkeiten, die außerhalb der Landwirtschaft nur einen geringen Wert haben.

- Notwendige Fähigkeiten in der Landwirtschaft können Eintrittsbarrieren für nicht-landwirtschaftliche Arbeitskräfte darstellen.

Ob Familienarbeitskräfte oder Lohnarbeitskräfte in der Landwirtschaft eingesetzt werden, hängt von den Opportunitätskosten der Familienarbeitskräfte im Verhältnis zum Lohnansatz für die Lohnarbeitskräfte ab. Familien- und Lohnarbeitskräfte sind vollständige Substitute, wenn die Opportunitätskosten für alle Familienarbeitskräfte den Lohnkosten für Lohnarbeitskräfte entsprechen.

\subsubsection{Die statistisch erfasste Beschäftigungssituation in der deutschen Landwirtschaft}

2.2.2.1 Methodische Vorbemerkungen $\mathrm{zu}$ den Veränderungen in der Arbeitskräfteerhebung

Die Daten in den folgenden Tabellen 4 bis 6 stammen aus der Erhebung der landwirtschaftlichen Arbeitskräfte im Rahmen der Agrarstrukturerhebung anhand von Stichprobenbetrieben. Bis 1995 wurden die Arbeitskräfte in den landwirtschaftlichen Betrieben in einer eigenständigen jährlichen Erhebung erfasst. Seit 1995 erfolgt die 
Erhebung der Arbeitskräfte in den landwirtschaftlichen Betrieben Deutschlands in einem zweijährigen Turnus innerhalb des Ergänzungsprogramms zur repräsentativen Agrarstrukturerhebung, in dem neben Sachverhalten zur Beschäftigung in den landwirtschaftlichen Betrieben auch weitere Strukturmerkmale wie z.B. Eigentums- und Pachtverhältnisse oder auch außerbetriebliche Erwerbs- und Unterhaltsquellen erfragt werden. Die repräsentative Agrarstrukturerhebung unterscheidet sich von der ebenfalls zweijährlich stattfindenden Agrarstrukturerhebung in der Anzahl der erhobenen Betriebe und im Umfang der erhobenen Merkmale (NAUSE 2003: 302). D.h., die Merkmale, die sowohl in der repräsentativen als auch in der regulären Agrarstrukturerhebung erhoben werden (z.B. die Anzahl der Betriebe, LF je Betrieb) weichen voneinander $\mathrm{ab}$, da sich diese Merkmale in der repräsentativen Agrarstrukturerhebung durch Hochrechnung ergeben.

Während des Betrachtungszeitraumes haben sich folgende weitere methodische Veränderungen bei der Erhebung ergeben:

- Seit 1999 haben sich die Erhebungseinheiten bei der Agrarstrukturerhebung verändert. Es werden nur noch Betriebe erfasst, deren landwirtschaftlich genutzte Fläche mindestens zwei ha beträgt (mindestens zehn ha Waldfläche bisher 1 ha). ${ }^{3}$

- Personengesellschaften werden erst seit 1997 eigenständig erfasst. Hierdurch kommt es $\mathrm{zu}$ einer Verzerrung bei der Darstellung der Beschäftigungsverhältnisse und bei der Bedeutung der Betriebsgruppen im Zeitablauf. Denn die Personengesellschaften stammen vor allem aus der Betriebsgruppe der Einzelunternehmen, mit der getrennten Erfassung der Personengesellschaften sinkt so die Zahl der Einzelunternehmen. Außerdem werden vormals als Familienarbeitskräfte gezählte Beschäftigte nun methodisch den familienfremden Arbeitskräften zugerechnet. Diese methodische Veränderung konnte bei der Bearbeitung der Tabellen nicht bereinigt werden, aus diesem Grunde sind die Veränderungen bei den nachgewiesenen Arbeitskräften zwischen den Jahren nicht direkt vergleichbar.

- Bis 1999 wurde die Zahl der Saisonarbeitskräfte nur für den Berichtszeitraum (April eines Jahres) nachgewiesen. Da landwirtschaftliche Betriebe in

\footnotetext{
${ }^{3}$ Für Betriebe mit Spezialkulturen oder Tierbeständen gelten Mindestgrößen, die der jährlichen Markterzeugung von 1 ha LF (bis 1997) bzw. 2 ha (ab 1999) entsprechen.
} 
Deutschland aber auch über den April hinaus Saisonarbeitskräfte beschäftigen (z.B. in der Spargel-, Getreide- und Hopfenernte oder Weinlese) und dadurch die tatsächliche Anzahl der in den landwirtschaftlichen Betrieben beschäftigten nichtständigen familienfremden Arbeitskräfte unterschätzt wurde, wurde der Erhebungszeitraum angepasst. Nun werden alle im Betrieb während des Zeitraum eines Jahres (Mai des Vorjahres bis April des Berichtsjahres) beschäftigte Saisonarbeitskräfte erhoben.

Die methodischen Veränderungen während des Zeitraums 1991-2005 führen dazu, dass einige Veränderungen auf verschiedene Erhebungsmethoden zurückzuführen sind.

\subsubsection{Die statistisch erfasste Beschäftigungssituation in den landwirtschaftlichen Betrieben des früheren Bundesgebiets}

Die Beschäftigungssituation weist in den landwirtschaftlichen Betrieben der neuen und alten Bundesländer deutliche Unterschiede auf. Dementsprechend werden diese Regionen im Folgenden differenziert behandelt.

Von 1991 bis 2005 ist die Zahl der landwirtschaftlichen Betriebe im früheren Bundesgebiet von 621.000 auf 360.400 Betriebe zurückgegangen. Dies entspricht einem Rückgang von $58 \%$ in 14 Jahren und 3,81\% jährlich. Ein Teil des Rückgangs der Anzahl landwirtschaftlicher Betriebe ist mit der Erhebungsmethodik zu begründen. Seit dem Jahr 1999 werden nur noch Betriebe mit einer landwirtschaftlichen Nutzfläche ab 2 ha erhoben. Die durchschnittlich landwirtschaftlich genutzte Fläche je Betrieb stieg im gleichen Zeitraum von 18,9 ha im Jahr 1991 auf rund 32 ha im Jahr 2005.

Bislang machen die Familienarbeitskräfte den deutlich überwiegenden Teil der in der Landwirtschaft Beschäftigten im früheren Bundesgebiet aus, doch ist ein Rückgang von jährlich 4,1 \% feststellbar. Betrachtet man den Zeitraum 1997-2005 isoliert, dann ergibt sich eine durchschnittliche jährliche Änderungsrate von -3,63\%. Das bedeutet, dass die Zurechnung der originär zur Familie gehörenden Arbeitskräfte in den Personengesellschaften zu den familienfremden Arbeitskräften bis 1997 nur einen geringen Einfluss auf den Rückgang der Anzahl der Familienarbeitskräfte hat.

1991 stellten die Familienarbeitskräfte noch über 88 \% aller landwirtschaftlichen Arbeitskräfte, 2005 waren es nur noch 66,91 \% (vgl. Tabelle 4). Zugleich nimmt die 
Bedeutung von Lohnarbeitskräften zu. Von 1991 bis 2003 stieg die Zahl der ständig beschäftigten familienfremden Arbeitskräfte um jährlich 1,62 \%. Sie stellten 1991 nur 5,46\% aller Arbeitskräfte, 2003 waren es schon 9,30\%. Wie in Tabelle 4 ersichtlich, zeigt die Gruppe der nicht ständig beschäftigten Lohnarbeitskräfte die höchste jährliche Wachstumsrate. Sie betrug im Durchschnitt der Jahre 1991 bis 2005 7,75 \%. Diese hohe Wachstumsrate ist jedoch durch den beschriebenen Methodenwechsel bei der Erhebung der Saisonarbeitskräfte durch das Statistische Bundesamt verzerrt.

TABELLE 4: AUSGEWÄHLTE ERGEBNISSE ZUR BESCHÄFTIGUNG IN DEN LANDWIRTSCHAFTLICHEN BETRIEBEN DES FRÜHEREN BUNDESGEBIETS

\begin{tabular}{|c|c|c|c|c|c|c|}
\hline & \multicolumn{6}{|c|}{$\begin{array}{l}\text { Früheres Bundesgebiet mit Berlin } \\
\text { Landwirtschaftliche Betriebe insgesamt }\end{array}$} \\
\hline & 1991 & 1995 & 1999 & 2003 & 2005 & $\begin{array}{l}\text { Durchschnittliche } \\
\text { jährliche Änderungs- } \\
\text { rate in \% }\end{array}$ \\
\hline $\begin{array}{l}\text { Betriebe } \\
\text { in } 1000 \text { Betriebe }\end{array}$ & 621 & 535,9 & 432,5 & 360,6 & 360,4 & $-3,81$ \\
\hline $\begin{array}{l}\text { LF zusammen } \\
1000 \text { ha LF }\end{array}$ & 11754,4 & 11637,6 & 11530,3 & 11475,5 & 11455,3 & $-0,18$ \\
\hline $\begin{array}{l}\text { LF je Betrieb } \\
\text { in ha }\end{array}$ & 18,9 & 21,7 & 26,7 & 31,8 & 31,8 & 3,79 \\
\hline $\begin{array}{l}\text { Familienarbeitskräfte in } 1000 \\
\text { Personen }\end{array}$ & 1337,3 & 1099,2 & 901,7 & 782,6 & 743,8 & $-4,10$ \\
\hline $\begin{array}{l}\text { Ständig beschäftigte } \\
\text { familienfremde Arbeitskräfte in } \\
1000 \text { Personen }\end{array}$ & 82,6 & 72 & 99,1 & 103 & 103,4 & 1,62 \\
\hline $\begin{array}{l}\text { Nicht ständig beschäftigte } \\
\text { familienfremde Arbeitskräfte in } \\
1000 \text { Personen }\end{array}$ & 93 & 74,3 & 233,1 & 251 & 264,4 & 7,75 \\
\hline $\begin{array}{l}\text { Anteil der Familienarbeitskräfte an } \\
\text { der Summe aller Arbeitskräfte } \\
\text { (nach Personen) in \% }\end{array}$ & $83,39 \%$ & $88,25 \%$ & $73,08 \%$ & $68,85 \%$ & $66,91 \%$ & $-1,97$ \\
\hline $\begin{array}{l}\text { Anteil der ständig beschäftigten } \\
\text { familienfremden Arbeitskräfte an } \\
\text { der Summe aller Arbeitskräfte } \\
\text { (nach Personen) in \% }\end{array}$ & $5,46 \%$ & $5,78 \%$ & $8,03 \%$ & $9,06 \%$ & $9,30 \%$ & 3,88 \\
\hline $\begin{array}{l}\text { Betriebliche Arbeitsleistung } \\
\text { insgesamt (in } 1000 \text { AK-Einheiten) }\end{array}$ & 705,9 & 571,1 & 499,6 & 483,5 & 458,5 & $-3,04$ \\
\hline $\begin{array}{l}\text { Betriebliche Arbeitsleistung je } 100 \\
\text { ha LF }\end{array}$ & 6 & 4,9 & 4,3 & 4,2 & 4,0 & $-2,85$ \\
\hline
\end{tabular}

Quelle: StAtistisches Bundesamt, Fachserie 3, Reihe 2.2 bzw. Reihe 2.18 (ab 1997), verschiedene Jahrgänge; eigene Berechnungen

Aufgrund der erst seit 1999 über das Jahr erfassten Anzahl der Saisonarbeitskräfte wird im Folgenden deren Entwicklung für den Zeitraum 1999-2005 betrachtet. Demnach ist 
eine jährliche Wachstumsrate der nicht ständig beschäftigten Lohnarbeitskräfte von $2,12 \%$ zu beobachten.

Bei der Erhebung der Familienarbeitskräfte und der ständigen familienfremden Arbeitskräfte unterscheidet die Agrarstrukturerhebung zwischen vollbeschäftigten und teilzeitbeschäftigten Arbeitskräften. Eine Besonderheit der landwirtschaftlichen Betriebe des früheren Bundesgebiets ist der relativ geringe Grad der Vollbeschäftigung mit betrieblichen Arbeiten unter den Familienarbeitskräften. So waren im Jahr 2005 nur $43 \%$ der Betriebsinhaber mit betrieblichen Arbeiten vollbeschäftigt. Bei den Familienangehörigen lag der Grad der Vollbeschäftigung hingegen nur bei 11,47 \% (STATISTISCHES BundeSAMT 2005). In den Haupterwerbsbetrieben liegt der Anteil der mit betrieblichen Arbeiten vollbeschäftigten Betriebsinhabern und Familienangehörigen wesentlich höher. Dies zeigt die Bedeutung der Nebenerwerbslandwirtschaft im früheren Bundesgebiet. Mit der Betriebsgröße nimmt auch der Anteil der Vollbeschäftigten zu. 55,3\% der ständig beschäftigten familienfremden Arbeitskräfte der landwirtschaftlichen Betriebe waren im Jahr 2005 vollbeschäftigt.

Die Analyse der in Tabelle 4 dargestellten Zahlen zeigt, dass der Familienbetrieb im früheren Bundesgebiet nach wie vor die dominierende Arbeitsverfassung ist. Lohnarbeitskräfte haben jedoch eine zunehmende Bedeutung für die landwirtschaftlichen Betriebe.

\subsubsection{Die statistisch erfasste Beschäftigungssituation in den landwirtschaftlichen Betrieben der neuen Bundesländer}

In Tabelle 5 ist die Beschäftigungssituation in den landwirtschaftlichen Betrieben der neuen Bundesländer dargestellt. Sie weist deutliche Unterschiede zum früheren Bundesgebiet auf.

In den neuen Bundesländern gab es 2005 ca. 29.500 Betriebe in denen insgesamt 165.100 Personen beschäftigt waren. Im Gegensatz zum früheren Bundesgebiet ist die Anzahl landwirtschaftlicher Betriebe steigend (2,2 \% jährliche durchschnittliche Wachstumsrate). Dies erklärt sich aus der Neu- oder Ausgründung landwirtschaftlicher Betriebe im Zuge der Umstrukturierung der ostdeutschen Landwirtschaft.

Die landwirtschaftlich genutzte Fläche pro Betrieb nahm im betrachteten Zeitraum ab. Durch die Aus- und Neugründung vor allem von Familienbetrieben sank die 
durchschnittliche Betriebsgröße in den neuen Bundesländern von 243,8 ha LF im Jahr 1991 auf 189,2 ha im Jahr 2005. Damit war der durchschnittliche Betrieb in den neuen Ländern immer noch fast siebenmal so groß wie der Durchschnittsbetrieb im früheren Bundesgebiet.

TABELLE 5: AUSGEWÄHLTE ERGEBNISSE ZUR BESCHÄFTIGUNG IN DEN LANDWIRTSCHAFTLICHEN BETRIEBEN IN DEN NEUEN LÄNDERN

Neue Länder

Landwirtschaftliche Betriebe insgesamt

\begin{tabular}{|c|c|c|c|c|c|c|}
\hline & 1991 & 1995 & 1999 & 2003 & 2005 & $\begin{array}{l}\text { Durchschnittliche } \\
\text { jährliche Änderungs- } \\
\text { rate in \%: }\end{array}$ \\
\hline $\begin{array}{l}\text { Betriebe } \\
\text { in } 1000 \text { Betriebe }\end{array}$ & 21,7 & 31 & 29,5 & 29,8 & 29,5 & 2,2 \\
\hline $\begin{array}{l}\text { LF zusammen } \\
\text { in } 1000 \text { ha LF }\end{array}$ & 5282,3 & 5519,4 & 5588,8 & 5552,1 & 5580 & 0,4 \\
\hline $\begin{array}{l}\text { LF je Betrieb } \\
\text { in ha }\end{array}$ & 243,8 & 178 & 189,7 & 186,3 & 189,2 & $-1,8$ \\
\hline $\begin{array}{l}\text { Familienarbeitskräfte } \\
\text { in } 1000 \text { Personen }\end{array}$ & 33,2 & 47,9 & 39,1 & 40,1 & 38,9 & 1,1 \\
\hline $\begin{array}{l}\text { Ständig beschäftigte } \\
\text { familienfremde Arbeitskräfte } \\
\text { in } 1000 \text { Personen }\end{array}$ & 321,2 & 106,2 & 96,9 & 88,4 & 84 & $-9,1$ \\
\hline $\begin{array}{l}\text { Nicht ständig beschäftigte } \\
\text { familienfremde Arbeitskräfte } \\
\text { in } 1000 \text { Personen }\end{array}$ & 7,6 & 7,4 & 32,9 & 38,2 & 42,2 & 13,0 \\
\hline $\begin{array}{l}\text { Anteil der Familienarbeitskräfte } \\
\text { an der Summe aller } \\
\text { Arbeitskräfte } \\
\text { in } \%\end{array}$ & $9,17 \%$ & $29,66 \%$ & $23,15 \%$ & $24,06 \%$ & $23,56 \%$ & 7,0 \\
\hline $\begin{array}{l}\text { Anteil der ständig beschäftigten } \\
\text { familienfremden Arbeitskräfte } \\
\text { an der Summe aller } \\
\text { Arbeitskräfte } \\
\text { in } \%\end{array}$ & $88,73 \%$ & $65,76 \%$ & $57,37 \%$ & $53,03 \%$ & $50,88 \%$ & $-3,9$ \\
\hline $\begin{array}{l}\text { Betriebliche Arbeitsleistung } \\
\text { insgesamt } \\
\text { in } 1000 \mathrm{AK} \text {-Einheiten }\end{array}$ & 312,4 & 127,3 & 112,8 & 104,9 & 100,6 & $-7,8$ \\
\hline $\begin{array}{l}\text { Betriebliche Arbeitsleistung } \\
\text { in AK-Einheiten je } 100 \text { ha LF }\end{array}$ & 5,9 & 2,3 & 2 & 1,9 & 1,8 & $-8,1$ \\
\hline
\end{tabular}

Quelle: Statistisches BundesAmt, Fachserie 3, Reihe 2.2 bzw. Reihe 2.18 (ab 1997) verschiedene Jahrgänge; eigene Berechnungen

Der Betrachtungszeitraum 1991 bis 2005 ist geprägt von einem drastischen Arbeitskräfterückgang in der Land- und Forstwirtschaft. Zugleich nahm im Gegensatz zum früheren Bundesgebiet die Zahl der beschäftigten Familienarbeitskräfte aufgrund 
der Wieder- und Neueinrichtung landwirtschaftlicher Familienbetriebe im Zeitraum von 1991 bis 2005 zu. Der Trend ist jedoch über den Betrachtungszeitraum nicht eindeutig.

Der Anteil der Familienarbeitskräfte an den in der Landwirtschaft beschäftigten Personen ist von 1991 bis 2005 von 9,1\% auf rund $24 \%$ gestiegen.

Im gleichen Zeitraum sank der Anteil der ständig beschäftigten Lohnarbeitskräfte an den landwirtschaftlichen Arbeitskräften von 88,73 \% auf 50,88 \%. Der Arbeitskräfterückgang betrifft also vor allem die Gruppe der ständig beschäftigten Lohnarbeitskräfte. Von 1991 bis 2005 ist deren Zahl um durchschnittlich 9,1 \% pro Jahr von 321.200 auf 84.000 Personen gesunken. Der Abbau von Lohnarbeitskräften in der ostdeutschen Landwirtschaft fand vor allem zu Beginn der 1990er Jahre statt. Von 1991 bis 1993 ist die Zahl der ständig beschäftigten Lohnarbeitskräfte um $37 \%$ zurückgegangen, von 1993 bis 2005 dann nochmals um weitere 10,6 \%. Dies ist auf folgende Veränderungen in der ostdeutschen Landwirtschaft nach der Wiedervereinigung zurückzuführen:

- Einführung arbeitssparender Technologien,

- Rückgang arbeitsintensiver Produktionsverfahren durch den Übergang zu EGMarktordnungen (ANDERS 2002: 38),

- Abbau der Bestände in der Tierproduktion (ebenda: 38),

- Aufdeckung verdeckter Arbeitslosigkeit (FOCK ET AL. 1996: 18),

- Auslagerung von Arbeitsbereichen nichtlandwirtschaftlichen Charakters, z.B. Baubrigaden, Werkstätten und Sozialeinrichtungen (BALMANN ET AL. 1996: 2, NAUSE: 2003: 312, FOCK ET AL. 1996: 18).

Der starke Abbau des Bestands an Lohnarbeitskräften trifft vor allem die landwirtschaftlichen Betriebe in der Rechtsform juristischer Personen. Die Zahl der in den Betrieben dieser Rechtsform beschäftigten Lohnarbeitskräfte sank von 316.100 Personen im Jahr 1991 auf 55.100 Personen im Jahr 2005. In den landwirtschaftlichen Betrieben der Rechtsform Einzelunternehmen stieg hingegen im Betrachtungszeitraum die Anzahl der Lohnarbeitskräfte von 5.100 auf 8.700 Personen an (STATISTISCHES BUNDESAMT, verschiedene Jahrgänge). Trotz der Zunahme an Lohnarbeitskräften in den Einzelunternehmen bei gleichzeitigem Rückgang der Lohnarbeitskräfte bei den 
juristischen Personen bleibt festzuhalten, dass der ganz überwiegende Teil der Lohnarbeitskräfte in den landwirtschaftlichen Betrieben der Rechtsform juristische Personen beschäftigt ist. Die juristischen Personen stellen also trotz des starken Arbeitsplatzabbaus immer noch die meisten Arbeitsplätze für landwirtschaftliche Arbeitnehmer. Die juristischen Personen bewirtschafteten 2005 im Durchschnitt 874,7 ha LF pro Betrieb, die Einzelunternehmen hingegen nur 62,7 ha LF je Betrieb (STATISTISCHES BundESAMT, verschiedene Jahrgänge). Da in den neuen Ländern vor allem große Betriebe ausbilden, ist der Rückgang dieser Betriebe vor dem Hintergrund der Ausbildungssituation als kritisch anzusehen (DAHMS 1998: 20).

Der Anteil der Vollbeschäftigten differiert nach der Art der Arbeitskräfte und der Rechtsform der Betriebe. Die Lohnarbeitskräfte sind zu einem überwiegenden Teil $(81,7 \%)$ vollbeschäftigt. Der Anteil der Vollbeschäftigten bei den Lohnarbeitskräften liegt damit deutlich höher als bei den Lohnarbeitskräften im früheren Bundesgebiet. Von den Betriebsinhabern waren im Jahr 2005 nur rund 34,3 \% vollbeschäftigt, bei den Familienangehörigen waren es 14,4\%.

Je 100 ha LF ist der Arbeitseinsatz in den neuen Ländern halb so hoch wie im früheren Bundesgebiet. Im Jahr 2005 wurden in den landwirtschaftlichen Betrieben des früheren Bundesgebiets 4 Arbeitskrafteinheiten ${ }^{4}$ (AKE) zur Bewirtschaftung von 100 ha LF benötigt, während es in den neuen Ländern nur 1,8 ha AKE pro 100 ha LF waren. Die geringere Arbeitsintensität in den ostdeutschen Betrieben ist u.a. auf die günstigere Betriebsgrößen- bzw. Bewirtschaftungsstruktur zurückzuführen.

Wie auch im früheren Bundesgebiet wird der überwiegende Teil der landwirtschaftlichen Betriebe in den neuen Bundesländern in der Rechtsform Einzelunternehmen bewirtschaftet. Im Jahr 2005 hatten die Betriebe der Rechtsform Einzelunternehmen einen Anteil von 77,97\% an den landwirtschaftlichen Betrieben der neuen Länder. Der Anteil der Betriebe in der Rechtsform juristische Personen sank von 1991 bis 2005 von fast $18 \%$ auf knapp $12 \%$. Während in der westdeutschen Landwirtschaft die landwirtschaftlichen Betriebe der Rechtsform juristische Personen nur eine zu vernachlässigende Bedeutung haben, sind die juristischen Personen für die ostdeutsche Landwirtschaft besonders wichtig, denn sie bewirtschaften fast $52 \%$ der

\footnotetext{
${ }^{4}$ Eine Arbeitskrafteinheit entspricht dem Arbeitseinsatz einer vollbeschäftigten Arbeitskraft bei voller Leistungsfähigkeit.
} 
landwirtschaftlich genutzten Fläche in den neuen Bundesländern und beschäftigen die meisten Arbeitskräfte. Wie auch im früheren Bundesgebiet hat die Rechtsform der Personengesellschaften eine wachsende Bedeutung in den neuen Bundesländern. Seit der erstmaligen Erhebung dieser Rechtsform im Jahr 1997 stieg der Anteil der Personengesellschaften von $9,38 \%$ auf $11,19 \%$ an. 


\subsubsection{Die statistisch erfasste Beschäftigungssituation in den landwirtschaftlichen Betrieben Deutschlands}

Die Ausführungen zu den beiden vorangegangenen Kapiteln haben gezeigt, dass sich die Beschäftigungssituation und die Agrarstruktur in den alten und neuen Bundesländern deutlich unterscheiden. Wie sich die statistische Gesamtsituation in Deutschland darstellt, beschreibt Tabelle 6 .

TABELLE 6: AUSGEWÄHLTE ERGEBNISSE ZUR BESCHÄFTIGUNG IN DEN LANDWIRTSCHAFTLICHEN BETRIEBEN DEUTSCHLANDS

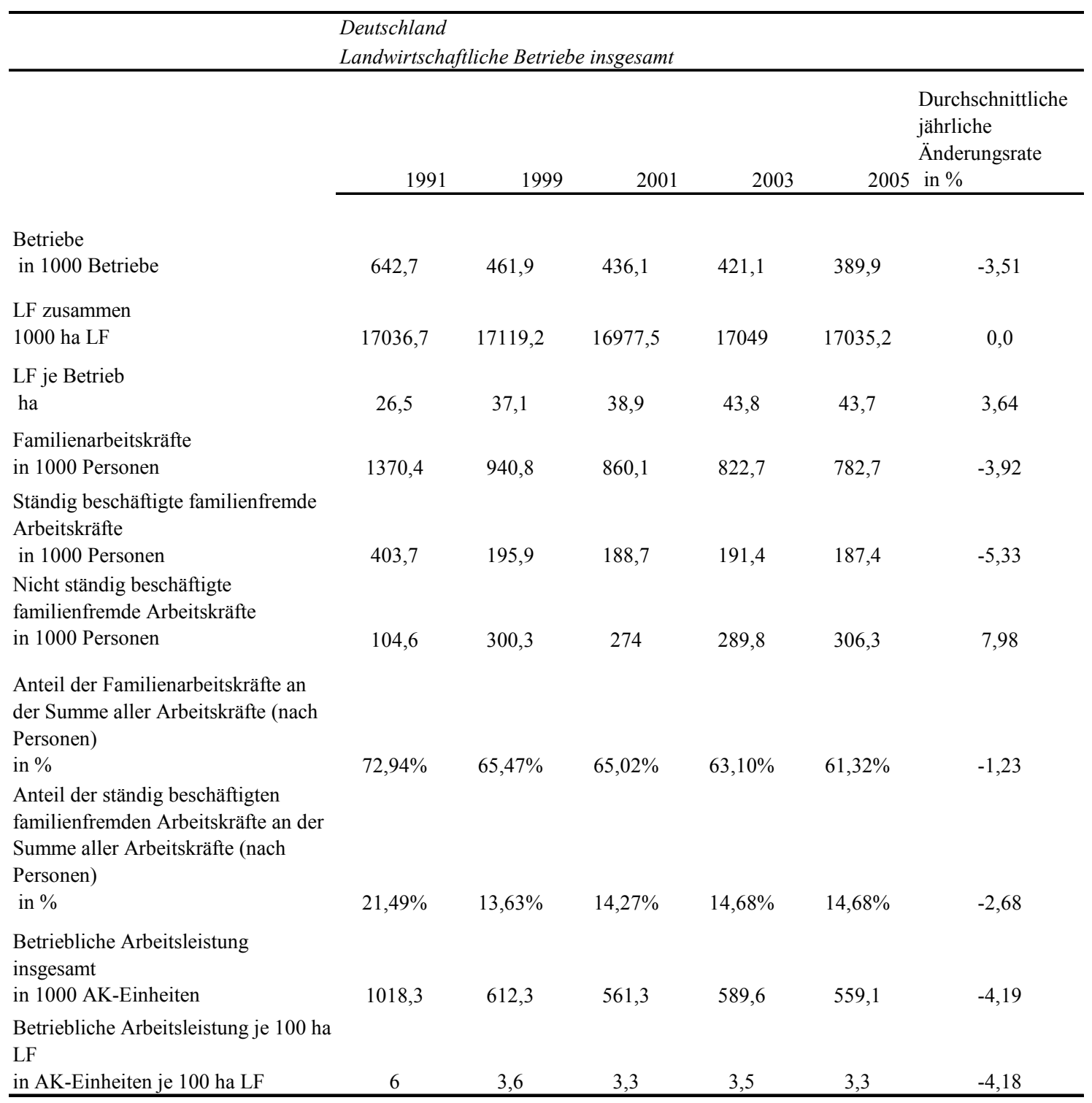

Quelle: Statistisches Bundesamt, Fachserie 3, Reihe 2.2. bzw. Reihe 2.18 (ab 1997) verschiedene Jahrgänge; eigene Berechnungen

In den landwirtschaftlichen Betrieben Deutschlands waren im Jahr 2005 zum Zeitpunkt der repräsentativen Agrarstrukturerhebung in den 389.900 landwirtschaftlichen 
Betrieben rund 1,276 Millionen Arbeitskräfte beschäftigt. Von diesen Arbeitskräften waren $61,32 \%$ Familienarbeitskräfte, 14,68 \% ständig beschäftigte Lohnarbeitskräfte und $24 \%$ Saisonarbeitskräfte. Gemessen in Arbeitskrafteinheiten erbrachten im Jahr 2005 die Familienarbeitskräfte $64 \%$, die ständig beschäftigen Lohnarbeitskräfte $24 \%$ und die Saisonarbeitskräfte $9 \%$ der Arbeitsleistung in den landwirtschaftlichen Betrieben (STATISTISCHES BUNDESAMT 2006: 28). Die Arbeit in den landwirtschaftlichen Betrieben Deutschlands wird folglich vornehmlich durch Familienarbeitskräfte verrichtet. Somit ist der Familienbetrieb in Deutschland nach wie vor die vorherrschende Organisationsform.

Von 1991 bis 2005 ist in Deutschland die Zahl der landwirtschaftlichen Betriebe um durchschnittlich 3,5\% pro Jahr zurückgegangen. Die gesamte landwirtschaftliche genutzte Fläche ist hingegen nicht gesunken. Als Folge hat sich die durchschnittliche landwirtschaftliche Nutzfläche auf den Betrieben von 26,5 ha im Jahr 1991 auf 43,7 ha im Jahr 2005 vergrößert.

Im betrachteten Zeitraum ist ferner die Zahl der in den landwirtschaftlichen Betrieben beschäftigten Familienarbeitskräfte jährlich um 3,9 \% zurückgegangen. Im gleichen Zeitraum ist die Zahl der ständig beschäftigten familienfremden Arbeitskräfte um über 4,3 \% jährlich zurückgegangen. Dies zeigt die höhere Mobilität der Lohnarbeitskräfte im Vergleich zu den Familienarbeitskräften. Zum einen haben Familienarbeitskräfte stärkere Substitutionsmöglichkeiten zwischen der Arbeit im Betrieb und der Arbeit im Haushalt bzw. der Freizeit als Lohnarbeitskräfte, zum anderen reagieren Lohnarbeitskräfte auch stärker als Familienarbeitskräfte auf die Veränderung von agrarund gesamtwirtschaftlichen Rahmenbedingungen (ANDERMANN UND SCHMITT 1996: 654). Es ist jedoch $\mathrm{zu}$ bedenken, dass der Rückgang der ständig beschäftigten familienfremden Arbeitskräfte wohl vor allem durch die Umstrukturierung der ostdeutschen Landwirtschaft bedingt ist. Der Rückgang bei den Familienarbeitskräften ist durch die Aufgabe von Familienbetrieben und das Ausscheiden von Familienarbeitskräften in den landwirtschaftlichen Betrieben des früheren Bundesgebiets begründet (NAUSE 2003: 307 f.). Außerdem ist ein Großteil der Familienarbeitskräfte nur mit einem Teil ihrer Arbeitszeit im Betrieb beschäftigt.

Die Änderungsrate bei den Saisonarbeitskräften ist verzerrt durch die Änderung der Erhebungsmethodik im Jahr 1999. Von 1991 bis 1997 sank die Zahl der 
Saisonarbeitskräfte um jährlich 3,7 \%. Von 1999-2005 stieg die Anzahl der nicht ständig beschäftigten Lohnarbeitskräfte um durchschnittlich $0,33 \%$. Für diesen betrachteten Zeitraum kann jedoch nicht von einem eindeutigen Trend gesprochen werden, da von 1999 bis 2001 die Zahl der Saisonarbeitskräfte zunächst sank und dann wieder anstieg. Neben der Verzerrung durch die unterschiedliche Erhebungsmethodik ist noch darauf hinzuweisen, dass die statistisch ausgewiesene Zahl der Saisonarbeitskräfte niedriger ausfällt, als tatsächlich in der Landwirtschaft beschäftigt sind. Bis zum Jahr 1999 war dieser Sachverhalt vor allem auf die Erhebungsmethodik der Agrarstatistik zurückzuführen. Hinzukommt, dass es den Betriebsleitern erst ab dem Jahr 1991 möglich war, legal osteuropäische Saisonarbeitskräfte, z.B. für Erntearbeiten einzusetzen (FASTERDING UND RIXEN 2005: 83). Die Vermittlung ausländischer Arbeitskräfte über die Zentralstelle für Arbeitsvermittlung (ZAV) ermöglicht einen Überblick über die Anzahl der angeforderten Saisonarbeitskräfte aus dem Ausland. FASTERDING UND RIXEN (2005: 84) stellten für das Jahr 2001 fest, dass auch nach der Veränderung der Erhebungsmethodik durch das Statistische Bundesamt die Anzahl der eingegangenen Anträge bei der ZAV, die Zahl der in der Agrarstatistik erfassten Saisonarbeitskräfte überstieg. Außerdem gibt es nach wie vor illegale Beschäftigungsverhältnisse bei Saisonarbeitskräften, die in der Agrarstatistik nicht erfasst sind (HESS 2002: 97).

Die betriebliche Arbeitsleistung je 100 ha LF ist in den Jahren 1991-2005 von 6 AKEinheiten je 100 ha LF auf 3,3 AK-Einheiten je 100 ha LF gesunken. Dieser Rückgang deutet auf eine bessere Auslastung der verfügbaren Arbeitskräfte durch die ansteigende landwirtschaftliche Nutzfläche je Betrieb hin. Außerdem ist der Einsatz arbeitssparender Technologien als weiterer Faktor zu nennen. 


\subsubsection{Prognosen zum Fachkräftebedarf in der Landwirtschaft}

In Industriegesellschaften ist ein Rückgang der Arbeitsleistung in den landwirtschaftlichen Betrieben um durchschnittlich 2-3 \% zu erwarten. Folgende Faktoren führen zum Rückgang der Beschäftigung in der Landwirtschaft:

- Das Engelsche Gesetz bedingt, dass in Industrienationen der Anteil der Lebensmittel an den Gesamtausgaben zurückgeht. Importe und Substitution heimischer Nahrungsmittel durch exotischere Nahrungsmittel führen $\mathrm{zu}$ abnehmendem Verbrauch heimischer Nahrungsmittel.

- Technischer Fortschritt führt dazu, dass Arbeit vermehrt durch Kapital ersetzt wird (ANDERMANN Und SCHMitt 1996: 631, BlAnC UND PERrIER-CoRnet 1999: 9).

- Die Verarbeitung von Rohstoffen wird zunehmend aus den landwirtschaftlichen Betrieben ausgelagert.

Im Folgenden soll zunächst auf die Altersstruktur der in der Landwirtschaft Beschäftigten eingegangen werden, da diese gemeinsam mit den Berufseintritten und austritten in vielen Analysen als wichtiger Bestimmungsgrund des zukünftigen Fachkräftebedarfs betrachtet wird (WIENER ET AL. 2004, FOCK UND FECHNER 2003, FASTERDING 2003: 350).

Abbildung 3 zeigt die Altersstruktur der ständig beschäftigten landwirtschaftlichen Lohnarbeitskräfte für das frühere Bundesgebiet und die neuen Länder. Es wird deutlich, dass die jüngeren Altersklassen zwischen 15 und 34 Jahren in den neuen Bundesländern deutlich schwächer besetzt sind als im früheren Bundesgebiet. Die Altersgruppen 35 bis 44 Jahre und stellen im früheren Bundesgebiet die meisten Lohnarbeitskräfte. In den neuen Bundesländern konzentriert sich das Alter der Beschäftigten auf die mittleren Altersgruppen. Betrachtet man lediglich die Altersgruppe 60 Jahre und älter, so könnte der Nachwuchsmangel im früheren Bundesgebiet größer sein. Ansonsten sind die Überalterungstendenz bei den Lohnarbeitskräften und die Dringlichkeit, Nachwuchskräfte zu gewinnen, in den neuen Bundesländern größer. 
ABBILDUNG 3: ALTERSSTRUKTUR DER STÄNDIG BESCHÄFTIGTEN LOHNARBEITSKRÄFTE IN DER LANDWIRTSCHAFT IM JAHR 2005

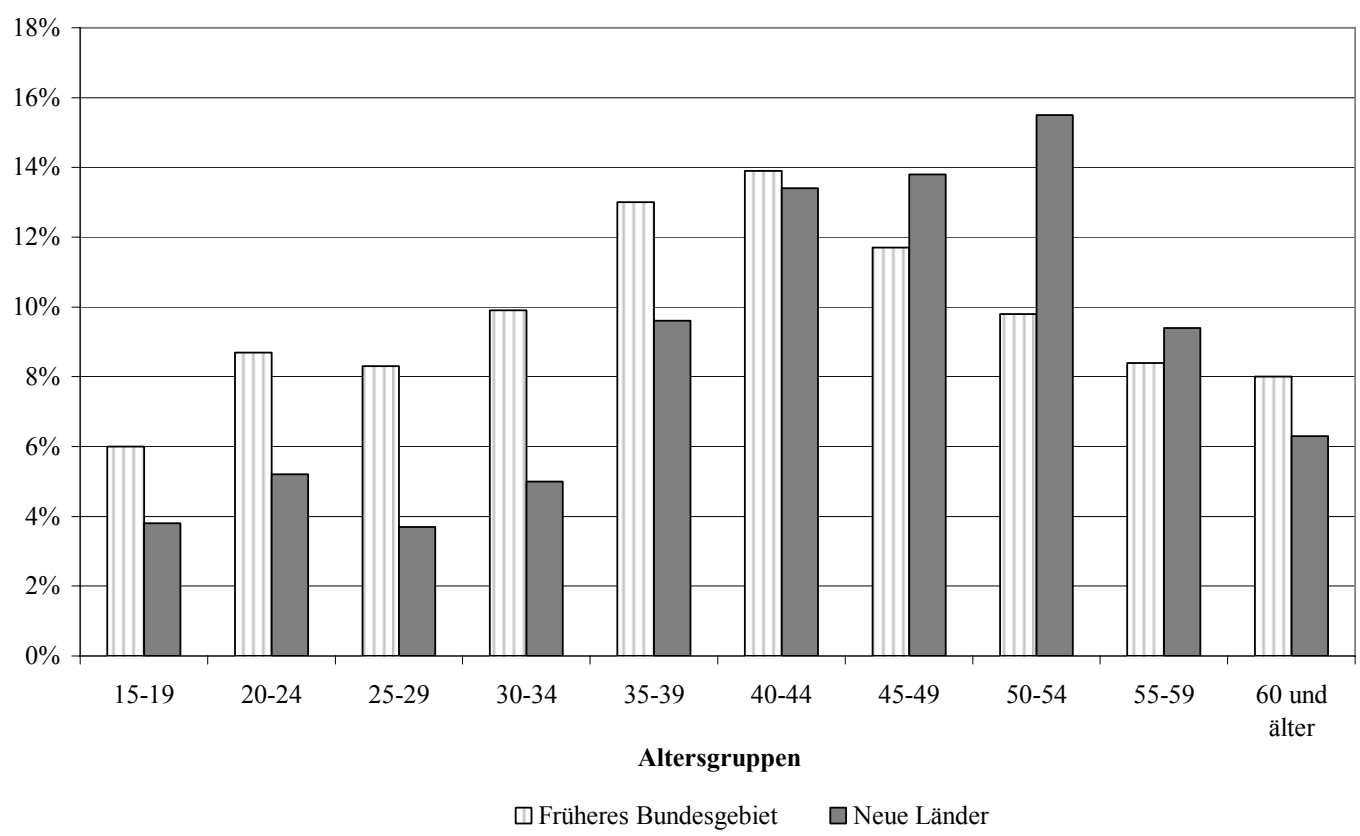

Quelle: StATISTISChES Bundesamt, Fachserie 3, Reihe 2.18

WIENER ET AL. (2004: 32 ff.) analysiert ergänzend die Altersstruktur der Lohnarbeitskräfte in den verschiedenen Rechtsformen. Im früheren Bundesgebiet sind demnach die Mitarbeiter der Unternehmen in der Rechtsform juristischer Personen besonders jung und die Mitarbeiter in den Unternehmen der Rechtsform natürlicher Personen besonders alt. In den neuen Bundesländern gilt das Gegenteil: Die Mitarbeiter in den Unternehmen der Rechtsform juristischer Personen sind hier im Durchschnitt erheblich älter.

Die Überalterung der Lohnarbeitskräfte in den neuen Bundesländern wird in vielen Studien als Indikator für einen sich ankündigenden Fachkräftemangel betrachtet (Fock Und Fechner 2003, DAHMS 1998, Wiener ET AL. 2004), da die Verrentungsphase der Mitarbeiter mit dem Berufseintritt geburtenschwacher Jahrgänge der Nachwendephase zusammenfällt. Die Überalterungstendenz betrifft nach den vorliegenden Analysen vor allem den Bereich der Führungskräfte (WIENER ET AL. 2004: 11).

Ein weiterer Hinweis auf einen drohenden Fachkräftemangel in der Landwirtschaft wird in den zitierten Studien in der geringen Ausbildungsbeteiligung landwirtschaftlicher Unternehmen gesehen. 
Die meisten Analysen zum Fachkräftemangel in der Landwirtschaft beziehen sich auf die neuen Bundesländern; sie sind teils bundeslandspezifisch durchgeführt worden. Ergebnisse für das frühere Bundesgebiet liegen aus dem IAB-Betriebspanel 1999 vor. In diesem Rahmen wurden Betriebsleiter nach den erwarteten Problemen aufgrund von Nachwuchsmangel befragt. Hier zeigte sich bei den Betrieben im früheren Bundesgebiet ein größeres Problem als bei den Betrieben in den neuen Bundesländern (FASTERDING 2003: $351 \mathrm{f}$ ).

Analysen, die auf Auswertungen der gemeldeten und nachgefragten Stellen der Arbeitsämter im früheren Bundesgebiet basieren, kommen zu einem anderen Ergebnis. Demnach gibt es dort keinen Fachkräftemangel in der Landwirtschaft, da die Anzahl der Arbeitsuchenden in den „grünen Berufen“ die der gemeldeten Stellen deutlich übersteigt und die Nachfrage der Landwirte nach den Bewerbern bei den Arbeitsämtern besonders gering ausfällt (KRÜGER 2005). Es ist jedoch fraglich, ob die Daten der Bundesagentur für Arbeit eine geeignete Datengrundlage zur Abschätzung des Fachkräftebedarfs darstellen, da nicht bekannt ist, wie viele Landwirte dem Arbeitsamt offene Stellen melden und/oder über diese Institution Mitarbeiter suchen. Es ist anzunehmen, dass viele Stellen in der Landwirtschaft auf anderen Wegen vergeben werden und gerade die Suche nach qualifizierten Arbeitskräften nicht über das Arbeitsamt geschieht.

Aus den vorliegenden Analysen lässt sich folglich keine eindeutige Prognose hinsichtlich des Fachkräftebedarfs ableiten. Im Rahmen der durchgeführten Betriebsleiterbefragung, die in Kapitel 5 vorgestellt wird, wurden die Betriebsleiter deshalb um eine Einschätzung des zukünftigen Fachkräftebedarfs für die Region und für den eigenen Betrieb gebeten. Auf einer fünfstufigen Skala konnten sie den Fachkräftebedarf von „stark sinkend“ bis „,stark steigend“ angeben. 
TABELLE 7: EINSCHÄTZUNG DES BETRIEBLICHEN FACHKRÄFTEBEDARFS ${ }^{5}$

\begin{tabular}{|c|c|c|c|c|c|}
\hline & $\begin{array}{c}\text { Ackerbau } \\
\mathrm{n}=76\end{array}$ & $\begin{array}{c}\text { Futterbau } \\
\mathrm{n}=45\end{array}$ & $\begin{array}{c}\text { Veredlung } \\
\mathrm{n}=20\end{array}$ & $\begin{array}{c}\text { Verbund } \\
\mathrm{n}=64\end{array}$ & $\begin{array}{c}\text { Gesamt } \\
\mathrm{n}=255\end{array}$ \\
\hline stark sinkend & $1,3 \%$ & $0 \%$ & $0 \%$ & $1,6 \%$ & $0,8 \%$ \\
\hline etwas sinkend & $35,5 \%$ & $24,4 \%$ & $5 \%$ & $31,3 \%$ & $27,1 \%$ \\
\hline gleich bleibend & $40,8 \%$ & $48,9 \%$ & $30 \%$ & $42,2 \%$ & $45,9 \%$ \\
\hline etwas steigend & $18,4 \%$ & $20 \%$ & $65 \%$ & $17,2 \%$ & $21,2 \%$ \\
\hline stark steigend & $2,6 \%$ & $6,7 \%$ & $0 \%$ & $7,8 \%$ & $4,8 \%$ \\
\hline
\end{tabular}

Quelle: Eigene Erhebung, Betriebsleiterbefragung

In Tabelle 7 ist die Einschätzung des betrieblichen Fachkräftebedarfs in Abhängigkeit von der betriebswirtschaftlichen Ausrichtung dargestellt. Es zeigt sich, dass die Betriebsleiter durchschnittlich mit einem gleich bleibenden betrieblichen Fachkräftebedarf rechnen. Es gibt jedoch einen hoch signifikanten Unterschied hinsichtlich der Prognose des zukünftigen Fachkräftebedarfs zwischen den verschiedenen betriebswirtschaftlichen Ausrichtungen. Während in $36,8 \%$ der untersuchten Ackerbau-, 24,4 \% der Futterbaubetriebe und 32,9 \% der Verbundbetriebe ein sinkender betrieblicher Fachkräftebedarf prognostiziert wird, gehen die Betriebsleiter der Veredlungsbetriebe bis auf eine Ausnahme von einem gleich bleibenden bis steigenden Bedarf aus. Hervorzuheben ist, dass $65 \%$ der befragten Betriebsleiter der Veredlungsbetriebe einen steigenden betrieblichen Fachkräftebedarf erwarten. Bei den Leitern von Acker- und Futterbaubetriebe ist es hingegen nur ein Viertel bis ein Drittel der Befragten, die einen steigenden Fachkräftebedarf prognostizieren. Dieses Ergebnis lässt sich wie folgt interpretieren: Im Acker- und Futterbau wirken Rationalisierungsmaßnahmen und der technische Fortschritt tendenziell arbeitssparend, während der technische Fortschritt in der Veredlung zwar auch rationalisierend wirkt, die Veredlung jedoch arbeitsintensiver ist.

Ein Betriebsleiter merkte an, dass es für ihn aufgrund der Arbeitsbedingungen (z.B. Geruchsbelästigung) schwierig sei, Mitarbeiter für den Schweinestall zu finden. Dies sei neben Rentabilitätsüberlegungen ein wichtiger Grund für die Aufgabe der Schweineproduktion gewesen. Der technische Fortschritt würde für junge Leute den Arbeitsplatz in der Pflanzenproduktion immer attraktiver machen. Seine Mitarbeiter

\footnotetext{
${ }^{5}$ Nicht gesondert ausgewiesen sind Betriebe, deren betriebswirtschaftliche Ausrichtung nicht eindeutig zu benennen war und die aus diesem Grund mehrere Antwortkategorien angegeben hatten.
} 
seien durch die Arbeit mit modernen Maschinen leicht für die Arbeit im Ackerbau zu begeistern (Betriebsleiter A).

Die Betriebsleiter aus den alten und neuen Bundesländern unterscheiden sich signifikant in Bezug auf die Prognose des betrieblichen Fachkräftebedarfs. Die Betriebsleiter der ostdeutschen Betriebe schätzen den Fachkräftebedarf eher sinkend und die westdeutschen Betriebsleiter eher als steigend ein. Vor dem Hintergrund der dargelegten Beschäftigungssituation in den alten und neuen Bundesländern ist diese Einschätzung nachvollziehbar. Der Personalbestand der Agrargenossenschaften befindet sich teilweise immer noch in der Abbauphase oder hat sich konsolidiert. Der zu Beginn der 90er Jahre drastische Personalabbau in den Agrargenossenschaften wurde von 90$95 \%$ der Betriebsleiter möglichst sozialverträglich durchgeführt (FECHNER 2006). D.h., es bestehen immer noch Einsparungspotenziale, die dazu führen, dass 50,9 \% der Betriebsleiter von den untersuchten Genossenschaften den Personalbedarf als sinkend einschätzen.

Die Einschätzung des regionalen Fachkräftebedarfs unterscheidet sich nicht wesentlich von der Prognose der betrieblichen Fachkräftebedarfs. Aus diesem Grunde, wird auf eine gesonderte Darstellung der regionalen Situation verzichtet.

Die Ergebnisse dieser (nicht repräsentativen) Studie zeigen, dass ein wachsender Fachkräftebedarf eher in den alten als in den neuen Bundesländern zu erwarten ist. Außerdem wird ein steigender Fachkräftebedarf für den Veredlungssektor prognostiziert. Offensichtlich planen hier die Betriebe eine Expansion und technischer Fortschritt scheint in den meisten Betrieben keine Arbeitskräfte zu substituieren.

Interessanterweise wird der Erfolg von Initiativen der berufständischen Vertretungen zur Stärkung der grünen Berufe, die einem zukünftigen Fachkräftemangel entgegenwirken sollen, zum Teil durch die Betriebsleitermentalität eingeschränkt. So unternimmt das Land Brandenburg eine Managementfortbildung für zukünftige Führungskräfte in der Landwirtschaft. Interessierte junge Leute mit einer entsprechenden landwirtschaftlichen Ausbildung haben die Möglichkeit, in einem landwirtschaftlichen Großbetrieb erste Betriebsleitererfahrungen $\mathrm{zu}$ sammeln und parallel durch Seminare Führungskompetenz zu erwerben. Leider gibt es inzwischen mehr Bewerber als freie Traineeplätze in den Betrieben, da viele Betriebsleiter nicht 
bereit sind, Nachwuchskräfte, die nicht aus der Nachbarschaft kommen und zu denen bereits ein persönliches Verhältnis besteht, aufzunehmen (FECHNER 2006).

Hinzukommt, dass es bei einigen Betriebsleitern eine Diskrepanz zwischen ihrem „gefühlten“ und tatsächlichen Alter gibt, die dazu führt, dass die Nachwuchsproblematik nicht früh genug wahrgenommen wird (ebenda).

\subsection{Arbeitsrechtliche Rahmenbedingungen}

Neben den wirtschaftlichen Rahmenbedingungen hat das Arbeitsrecht eine wichtige Bedeutung für die Betriebsleiter. Arbeitsrechtliche Rahmenbedingung beeinflussen die Entscheidung für oder gegen die Beschäftigung familienfremder Arbeitskräfte, die Art des Beschäftigungsverhältnisses und die Ausgestaltung der betrieblichen Anreiz- und Entgeltsysteme maßgeblich. BOKELMANN (2000: 209) spricht in diesem Zusammenhang von einer „Verrechtlichung der Arbeitsbeziehung“. Auch die Handlungsempfehlungen für die landwirtschaftliche Praxis müssen sich im Rahmen der arbeitsrechtlichen Möglichkeiten bewegen. Im folgenden Abschnitt werden daher in Kürze wichtige arbeitsrechtliche Rahmenbedingungen für die Gestaltung von Anreiz- und Entlohnungssystemen vorgestellt.

\subsubsection{Individuelles Arbeitsrecht}

Das Individualarbeitsrecht regelt die privatrechtlichen Beziehungen zwischen Arbeitnehmer und-geber.

Es existiert keine gesetzliche Begriffsbestimmung für den Begriff des Arbeitnehmers, die Zugehörigkeit zum Personenkreis der Arbeitnehmer wird nach dem Grad der persönlichen Abhängigkeit bestimmt. So führt der Arbeitnehmer eine fremdbestimmte Tätigkeit in persönlicher Abhängigkeit aus (RICHARDI 2006: XIII, MARTENS 1993: 152).

Der Vertragspartner des Arbeitnehmers ist der Arbeitgeber. Er kann von einem anderen unselbständige Tätigkeiten und Dienstleistungen aufgrund eines Vertrages verlangen. Arbeitgeber kann eine natürliche, juristische Person oder eine Personenhandelsgesellschaft sein; nicht aber der Konzern - da er nicht rechtsfähig ist jedoch eine Konzerntochtergesellschaft (MARTENS 1993: 152). 


\subsubsection{Arbeitsvertragsrecht}

Das Arbeitsverhältnis unterliegt der Privatautonomie und seine wichtigste Rechtsquelle ist das Bürgerliche Gesetzbuch. Die $\S \S 611-630$ BGB regeln den Dienstvertrag und die Rechten und Pflichten von Arbeitgeber und -nehmer:

- Der Arbeitsvertrag bedarf keiner besonderen Form. Er kann jedoch in verschiedenen Fällen nichtig werden, nämlich bei fehlender Geschäftsfähigkeit, Formmangel oder Sittenwidrigkeit (MARTENS 1993: 158).

- Der Arbeitnehmer schuldet dem Arbeitgeber die vereinbarte Dienstleistung. Der Arbeitgeber schuldet dem Arbeitnehmer im Gegenzug für die geleisteten Dienste die vereinbarte Vergütung (§ 611 Absatz 1).

- Damit der Arbeitsvertrag zustande kommt, müssen Art und Umfang der Dienstleistung sowie die Vergütung verabredet sein.

- Ist die Höhe der Vergütung nicht bestimmt, so ist eine übliche Vergütung als vereinbart anzusehen ( $(612$ Absatz 2).

- Auch im Falle eines Arbeitsausfalls oder wenn der Arbeitgeber den Arbeitnehmer nicht beschäftigen will, hat der Arbeitnehmer Anspruch auf die vereinbarte Vergütung (§ 615).

- Der Arbeitgeber trägt außerdem die Fürsorgepflicht für Gesundheit und Leben des Arbeitgebers ( $\S 617-619)$.

Die Regelungen des BGB zum Dienstvertrag regeln außerdem die Kündigungsfristen bei Dienstverhältnissen und die Rahmenbedingungen der Kündigung (§§ 621-630). Für befristete Arbeitsverhältnisse gilt das Teilzeit- und Befristungsgesetz (TzBfG).

\subsubsection{Arbeitsschutzrecht}

Das Arbeitsschutzrecht umfasst alle Normen, die der Staat zum Schutze des Arbeitnehmers vor den Gefahren des Arbeitslebens erlassen hat. Hier zu zählen z.B. das Arbeitszeitgesetz (ArbZG), die Arbeitsstättenverordnung, das Jugendarbeits- und Mutterschutzgesetz und das Bundesurlaubsgesetz. 
Aufgrund der zum Teil sehr langen und unregelmäßig anfallenden Arbeitszeiten ist die Arbeitszeitordnung für die Landwirtschaft und die Gestaltung von Anreizsystemen von besonderer Bedeutung.

Das Arbeitszeitgesetz (ArbZG), vom 06.06.1994, zuletzt geändert am 31.10.2006, soll die Sicherheit und den Gesundheitsschutz der Arbeitnehmer bei der Arbeitszeitgestaltung gewährleisten und die Rahmenbedingungen für flexible Arbeitszeiten verbessern $(\S 1$ Nr. 1). Zweck ist es weiterhin, den Sonntag und die staatlich anerkannten Feiertage als Tage der Arbeitnehmerruhe und der seelischen Erholung der Arbeitnehmer zu schützen ( $\$ 1$ Nr. 2).

Die tägliche Höchstarbeitzeit beträgt acht Stunden. Eine Verlängerung auf zehn Stunden täglich ist möglich, wenn während eines Zeitraums von sechs Kalendermonaten oder 24 Wochen die durchschnittliche Arbeitszeit von acht Stunden pro Werktag nicht überschritten wird ( 33 ArbZG).

Den Arbeitnehmern sind festgelegte Ruhepausen je nach der Dauer ihrer täglichen Beschäftigung zu gewähren.

Die Gestaltung des Arbeitszeitschutzes im Einzelnen überträgt der Gesetzgeber weitestgehend den Tarifvertragsparteien (RICHARDI 2006: XXXIII).

Das Arbeitszeitschutzgesetz trifft ferner eine abweichende Regelung für die Landwirtschaft bezüglich der täglichen Höchstarbeitszeit in der Bestell- und Erntezeit und erlaubt die Anpassung an die Witterungsgegebenheiten ( $\$ 7$ Absatz 2 Nr. 2), sofern die Gesundheit des Arbeitnehmers nicht gefährdet ist.

Ausnahmen vom Verbot der Sonn- und Feiertagsarbeit ( $\$ 9$ ArbZG) gibt es für die Landwirtschaft und in der Tierhaltung sowie in Einrichtungen zur Behandlung und Pflege von Tieren, wenn die Arbeiten nicht an Werktagen vorgenommen werden können ( $\$ 10$ Absatz 1 Nr. 12). Die Arbeitnehmer müssen jedoch innerhalb eines Zeitraums von 15 Tagen einen Ersatzruhetag für den gearbeiteten Sonn- oder Feiertag erhalten und die Regelungen zur Höchstarbeitzeit und Ausgleichszeiträume dürfen nicht überschritten werden (§11 Absatz 3). 
Abweichend von dieser Regelung kann im Rahmen eines Tarifvertrags oder einer Betriebsvereinbarung ein Ausgleichszeitraum für die an Sonn- und Feiertagen gearbeiteten Tage festgelegt werden $(\S 12)$.

Diese Regelung ist für die Landwirtschaft von großer Tragweite, denn in den Arbeitsspitzen ist es betrieblich oft nicht möglich, den Ersatzruhetag innerhalb eines Zeitraumes von 15 Tagen zu erteilen. Die Einrichtung eines Arbeitszeitkontos und der Ausgleich der geleisteten Arbeitstage in arbeitsärmeren Zeiten entsprechen den betrieblichen Erfordernissen häufig eher. Eine Analyse zu den Einsparungsmöglichkeiten bei den Personalkosten in Ackerbaubetrieben von MANN ET AL. (2006: 30) zeigt, dass in der Mehrzahl der untersuchten Betriebe die Überstunden im Winter ausgeglichen werden oder der Jahresurlaub im Winter genommen wird. Die Autoren weisen aber darauf hin, dass die teilweise üblichen Entlassungen der Mitarbeiter im Winter (DIPPMANN 2004: 33) oder die ausschließliche Verlagerung des Urlaubs in die arbeitsarmen Monate zu großer Unzufriedenheit bei den Mitarbeitern führen können (MANN ET AL. 2006: 30). Die in Kapitel 5 ausführlich dargestellte Betriebsleiterbefragung zeigt, dass in 54,6\% der untersuchten Unternehmen Arbeitszeitkonten geführt werden und 81,2\% der Betriebsleiter geleistete Überstunden „,abbummeln“ lassen (siehe Anhang, Betriebsleiterbefragung, Frage 21).

\subsubsection{Kollektives Arbeitsrecht}

Das kollektive Arbeitsrecht regelt die Existenz, Organisation und Funktion der arbeitsrechtlichen Vertretungsorgane (z.B. Betriebsräte, Gewerkschaften und Arbeitgeberverbände).

\subsubsection{Tarifvertragsrecht}

Das deutsche Arbeitsrecht ist geprägt vom Grundsatz der sozialen Selbstverwaltung. Hierzu zählen die Koalitionsfreiheit und die Tarifautonomie (RICHARDI 2006: XXXIV).

Das Tarifvertragsgesetz (TVG) regelt Inhalt und Form des Tarifvertrags. Dieser besteht aus einem schuldrechtlichen Teil, der die Rechte und Pflichten der Tarifvertragsparteien bestimmt und dem normativen Teil, in dem Inhalt, Abschluss und Beendigung von Arbeitsverhältnissen geregelt werden (EBENDA: XXXV). 
Im Unterschied zum Arbeitsvertrag bedürfen Tarifverträge der Schriftform, die Tarifvertragsparteien sind Gewerkschaften sowie Arbeitgeberverbände und einzelne Arbeitgeber.

Für Tarifverträge gilt das Unabdingbarkeitsprinzip, d.h. seine Rechtsnormen gelten unmittelbar und zwingend. Ausnahmen hiervon sind die Gestattung von abweichenden Vereinbarungen im Tarifvertrag oder veränderte Regelungen zugunsten des Arbeitnehmers (Günstigkeitsprinzip).

Manteltarifverträge regeln die allgemeinen Arbeitsbedingungen, während die Höhe der Löhne und Entgelte in Vergütungs- und Entgelttarifverträgen geregelt ist. Tarifverträge haben Vorrang vor Betriebsvereinbarungen, sind jedoch gegenüber den Gesetzen die rangniedere Rechtsquelle (EISENFÜHR UND THEUVSEN 2004: 188-189).

Tarifgebunden sind die Mitglieder der Vertragsparteien und die Arbeitgeber, die selbst eine Vertragspartei darstellen (§ 3 Absatz 1).

In den Tarifgebieten Mecklenburg-Vorpommern, Schleswig-Holstein, Hamburg und Niedersachsen bestehen Tarifverträge mit einem Leistungsbezug nur in der Entgeltgestaltung für die Melker in Schleswig-Holstein und Niedersachsen und für die Außendienstmitarbeiter der Rinderzucht Schleswig-Holstein (WEIDEL 2006).

Der Tarifvertrag über die Vergütung von Außendienstmitarbeitern der Rinderzucht Schleswig-Holstein eG vom 01.09.2002 sieht das folgende Entlohnungsschema vor:

- Die Außendienstmitarbeiter erhalten eine Grundvergütung von $50 \%$ der Entgeltgruppen III bzw. IV des Entgelttarifvertrages der RSH. Nach Vollendung des 50. Lebensjahres erhalten die Mitarbeiter $55 \%$ der Entgeltgruppe IV als Grundvergütung. Die Grundvergütung gilt 3.015 Besamungen bei einem Index von 1,8 ab $(\S 2$ Nr. 1$)$.

- $\mathrm{Ab}$ der 3016. Besamung erhielten die Mitarbeiter zum September 2002 4,35€ pro Besamung. Die Anpassung des Betrages erfolgt nach den jährlichen Tarifabschlüssen (§ 2 Nr. 2).

- Außerdem sieht der $\S 2$ einen so genannten Zonenzuschlag vor (Nr. 4), der als Ausgleich für einen erhöhten Zeitaufwand durch schwierige Verkehrswege, eine 
geringe Kuhbestandsdichte oder das Zuchtniveau vorgesehen ist. Es werden im oben genannten Tarifvertrag drei verschiedene Stufen der Ausgleichszahlung ausgewiesen.

- Für durchgeführte und abgerechnete Trächtigkeitsuntersuchungen erhalten die Techniker einen Stücklohn von 1,02€ (§6).

- Zusätzliche Prämien gibt es für die vom Tierzuchttechniker angemeldeten und verkauften Tiere in Höhe von $5,11 €(\S 7)$ und für die Neuanwerbung von Betrieben eine Zahlung von 2,6€ pro Besamung für die Dauer von 2 Jahren ( $\S$ 8).

Für die Melker in Schleswig-Holstein sieht der Lohntarifvertrag vom 01.01.2000 die folgenden Prämien vor (§ 3), die zusätzlich zum tarifvertraglich geregelten Grundlohn bezahlt werden:

- Eine Milchleistungsprämie differenziert nach der Anzahl der melkenden Personen je volle 100 Einheiten Fett und Eiweiß (FE) (Nr. A).

- Eine Abkalbeprämie für Betriebe mit überwiegender Abkalbung aus dem eigenen Bestand von 14 DM für jedes innerhalb von 13 Monaten nach dem letzten Abkalben lebend geborene Kalb (Nr. B).

- Eine Aufzuchtprämie in den ersten drei Lebensmonaten des Kalbes von 8 DM je Kalb (Nr. C).

- Prämien für den Verkauf von Tieren gestaffelt nach Schlachtkalb, Jungvieh und Großvieh zum Schlachter sowie Zucht- und Nutzvieh.

\subsubsection{Betriebsverfassungsrecht und Mitbestimmungsrechte der Arbeitnehmer}

Das Betriebsverfassungsgesetz regelt die betriebliche Mitbestimmung der Arbeitnehmer.

Die Gruppe der Arbeitnehmer wird vom Betriebsrat repräsentiert. Für Arbeitgeber und Betriebsrat ist eine vertrauensvolle Zusammenarbeit geboten (RICHARDI 2006: XVII). 
Die Größe des Betriebsrates hängt von der Anzahl der wahlberechtigten Arbeitnehmer des Betriebes ab. ${ }^{6}$ Dem Betriebsrat kommen allgemeine Aufgaben ( $\S 80$ BetrVG Absatz 1) und besondere Aufgaben zu. Zu den allgemeinen Aufgaben des Betriebsrates gehört es, auf die Einhaltung der Gesetze und Regelungen zugunsten der Arbeitnehmer $\mathrm{zu}$ achten. $\mathrm{Zu}$ den besonderen Aufgaben gehören die sozialen, personellen und wirtschaftlichen Angelegenheiten, hier hat der Betriebsrat Mitwirkungs- und Mitbestimmungsrechte (RICHARDI 2006: XVII).

Die Fragen der betrieblichen Lohngestaltung, insbesondere die Einführung von Entlohnungsgrundsätzen, die Einführung, Anwendung oder Änderung von neuen Entlohnungsmethoden sowie die Festsetzung von Akkord- und Prämiensätzen gehören zu den sozialen Angelegenheiten ( $§ 87$ Nr. 10 und 11 BetrVG).

D.h., für landwirtschaftliche Betriebe, die einen Betriebsrat haben, ist die Lohngestaltung nicht alleine durch die Betriebsleitung festzulegen. Nach Einschätzung der IG Bauen-Agrar-Umwelt gibt es in Deutschland derzeit 100 Betriebsräte in der originären Landwirtschaft (HEIL 2007). Folglich spielen Betriebsräte in der Landwirtschaft eine sehr geringe Rolle und nur wenige landwirtschaftliche Lohnarbeitskräfte werden in ihren Interessen durch Betriebsräte vertreten.

Neben dem, durch das Arbeitsrecht begründeten, betrieblichen Mitbestimmungsrecht der Arbeitnehmer kommt in bestimmten Rechtsformen noch das unternehmerische Mitbestimmungsrecht zur Geltung. In einigen Unternehmen wird den Arbeitnehmern eine Beteiligung am Aufsichtsrat zu einem Drittel eingeräumt. Diese Beteiligung regelt das Drittelbeteiligungsgesetz (DrittelbG) vom 18.05.2004. Es betrifft alle Kapitalgesellschaften ab 500 Arbeitnehmern und die Aktiengesellschaften und KGaA unter 500 Arbeitnehmern, sofern es sich nicht um Familienunternehmen handelt. Ausgeschlossen von diesem Gesetz sind ferner die so genannten Tendenzbetriebe (z.B. Unternehmen mit politischer, konfessioneller, karitativer oder wissenschaftlicher Bestimmung) und die Unternehmen der Montanunternehmen. Für letztere finden nämlich das Mitbestimmungsgesetz der Montanindustrie oder das MontanMitbestimmungsergänzungsgesetz Anwendung (§ 1 DrittelbG).

$6 \mathrm{Zu}$ den Arbeitnehmern im Sinne des Betriebsverfassungsgesetzten gehören Mitglieder der Unternehmensleitung. Der Personenkreis, der nach dem BetrVG kein Arbeitnehmer ist, wird detailliert in $\S 5$ beschrieben. 
Nach der Agrarstrukturerhebung 2005 wurden im betreffenden Jahr 4.900 landwirtschaftliche Betriebe in der Rechtform juristischer Personen bewirtschaftet. In diesen Betrieben waren insgesamt 64.000 ständig beschäftigte Lohnarbeitskräfte beschäftigt. Der überwiegende Teil dieser Betriebe verfügt über mehr als 100 ha LF. 1.100 dieser Betriebe hatten mehr als 1.000 ha LF und benötigten für die Bewirtschaftung durchschnittlich 32,19 ständige Lohnarbeitskräfte. Daraus lässt sich schlussfolgern, dass der überwiegende Teil der Arbeitnehmer in den juristischen Personen nicht vom Drittelbeteiligungsgesetz betroffen ist, da nur eine geringe Zahl der Kapitalgesellschaften in der Landwirtschaft mehr als 500 Arbeitnehmer beschäftigen wird. Die AG und KGaA spielen in der Landwirtschaft eine geringe Rolle. Dies zeigt auch die Bedeutung der verschiedenen Rechtsformen in der Stichprobe der Betriebsleiterbefragung, die in Kapitel 5.2.1 dargestellt wird.

In diesem Kapitel wurden einige wichtige Rahmenbedingungen für die leistungsorientierte Lohngestaltung in der Landwirtschaft dargestellt. Es lässt sich festhalten, dass landwirtschaftliche Produktionsprozesse von stochastischen Größen beeinflusst werden, die die objektive Messbarkeit des individuellen Leistungsbeitrags der Mitarbeiter erschweren. Außerdem resultieren aus den Eigenheiten der agrarischen Produktion Flexibilitätserfordernisse an die Arbeitsorganisation und punktuell hohe Arbeitsbelastungen. In landwirtschaftlichen Familienbetrieben sind starke Verflechtungen zwischen Haushalt und Familie vorzufinden. Altruismus und Loyalität prägen in vielen Fällen die Arbeitshaltung der Familienmitglieder. Trotz der hohen Arbeitsbelastung sind Landwirte sehr zufrieden mit ihrer Tätigkeit. Sie bietet direktes Feedback und fördert dadurch intrinsische Motivation, die eine zentrale Stellung in der Frage nach der optimalen Anreizgestaltung einnimmt, wie das folgende Kapitel zeigen wird. Die genannten Besonderheiten der Agrarproduktion spiegeln sich zum Teil auch in der Agrarstatistik wider. So lässt sich z.B. die überdurchschnittliche Arbeitsbelastung statistisch nachweisen und kann gemeinsam mit vergleichsweise geringen Löhnen ein Hindernis für die Motivation, Bindung und Anwerbung von Lohnarbeitskräften darstellen. Diese erlangen eine wachsende Bedeutung für die deutsche Landwirtschaft, wie die Auswertung der statistischen Daten verdeutlichen. Neben den biologisch und klimatisch bedingten Grenzen der Leistungsentlohnung in der Landwirtschaft sind aber auch arbeitsrechtliche Restriktionen $\mathrm{zu}$ beachten, wie im letzten Abschnitt dieses Kapitels erläutert wurde. 


\section{Leistungsorientierte Entlohnung im theoretischen Kontext}

Die natürlichen, wirtschaftlichen und rechtlichen Verhältnisse stellen die Rahmenbedingungen für die Gestaltung von Anreizsystemen dar und begrenzen die Ausgestaltungsmöglichkeiten und Anwendungsgebiete leistungsorientierter Entlohnungssysteme für den landwirtschaftlichen Unternehmer. Um zu einem Urteil über die Anwendungsbereiche und -möglichkeiten leistungsorientierter Entlohnung in der deutschen Landwirtschaft zu kommen, ist darüber hinaus eine Analyse der Theorieansätze zur optimalen Gestaltung von Anreizsystemen und $\mathrm{zu}$ den Erfolgsbedingungen leistungsorientierter Entlohnung notwendig.

Mit der Frage der Gestaltung von Entlohnungssystemen beschäftigen sich verschiedene Strömungen der betriebswirtschaftlichen Forschung. Im folgenden Kapitel wird eine Auswahl wichtiger theoretischer Ansätze zur Gestaltung von Anreizsystemen dargestellt.

Die Einordnung der verschiedenen theoretischen Ansätze ist nicht immer trennscharf, denn die Personalwirtschaftslehre befindet sich im Spannungsfeld verschiedener Theorien, die sich zumeist in ihren Einschätzungen zum Menschenbild und in ihrer Forschungsmethodik unterscheiden aber auch überschneiden und sich gegenseitig beeinflussen.

Zunächst soll ein Überblick darüber gegeben werden, wie verschiedene theoretische Strömungen der Betriebswirtschaftslehre diese Problemstellung bisher bearbeitet haben. Neben einer kurzen Einführung in die theoretischen Grundansätze der verschiedenen Theorien sollen auch die Grenzen der verschiedenen theoretischen Ansätze zur Lösung des Problems der optimalen Gestaltung von Anreizsystemen aufgezeigt werden.

Anschließend werden die Anforderungen an leistungsorientierte Entlohnungssysteme dargestellt, die erfüllt sein müssen, damit diese mit Erfolg eingesetzt werden können. 


\subsection{Institutionen- und personalökonomische Forschungsansätze}

Das nächste Kapitel widmet sich zwei institutionenökonomischen und einem personalökonomischen Forschungsansatz, die für die Gestaltung von materiellen Anreizsystemen von Bedeutung sind. Der Prinzipal-Agenten-Theorie ist in diesem Kapitel mehr Platz als den anderen beiden Ansätzen eingeräumt, um ihrer Relevanz gerade für die leistungs- und erfolgsorientierte Vergütung genüge zu tun.

\subsubsection{Prinzipal-Agenten-Theorie}

Neoklassischen Forschungsansätzen liegt das Menschenbild des rational handelnden eigennützigen homo oeconomicus zugrunde. Modifikationen lassen begrenzte Rationalität und Informationsasymmetrien zu. Zählt man diese Erweiterungen zur neoklassischen Ökonomie und betrachtet sie nicht als eigenständigen Forschungsansatz, was nach BRANDES ET AL. (1997) und SHAPIRO UND STIGLITZ (1984) zulässig ist, dann ist die Prinzipal-Agenten-Theorie (PA) wohl einer der prominentesten neoklassischen Ansätze zur Lösung von Anreizproblemen.

Die Prinzipal-Agenten-Theorie ist zusammen mit der Theorie der Verfügungsrechte (Property-Rights-Theorie) und der Transaktionskostentheorie der Neuen Institutionenökonomik zuzuordnen, die sich mit der Analyse von Institutionen (Märkte, Verfügungsrechte, Verträge, Hierarchien) beschäftigt (РіCOT 1991: 144 ff.)

Die Prinzipal-Agent-Theorie beschäftigt sich mit Delegationsbeziehungen bzw. -verträgen zwischen (im Normalfall) einem Auftraggeber bzw. einer Instanz (Prinzipal) und einem Auftragnehmer (Agent). Das Vorhandensein von Hierarchiebeziehungen zwischen den beiden Vertragspartnern unterscheidet u.a. die Prinzipal-Agenten-Theorie von der Spieltheorie.

Eine wesentliche Annahme der Prinzipal-Agenten-Theorie ist das Vorliegen von Informationsasymmetrien zwischen dem Auftraggeber und dem Auftragnehmer und darüber hinaus eine implizite Annahme von opportunistischem Verhalten des Auftragnehmers (ODENING 1994: 271). Durch die Unterstellung von Informationsasymmetrien, die opportunistisch von Seiten des Agenten ausgenutzt werden können, wird das neoklassische Menschenbild des vollständig informierten, rational handelnden homo oeconomicus erweitert. 
Hinsichtlich des Informationsstandes der Akteure kann zwischen sicherer und unsicherer Information unterschieden werden. Sichere Information liegt vor, wenn keine stochastischen Elemente auftreten. Demzufolge liegt unsichere Information vor, wenn stochastische Elemente auftreten.

Es werden die folgenden Formen von Informationsasymmetrien in der PrinzipalAgenten-Theorie unterschieden: Unter dem Begriff hidden action wird die Möglichkeit verborgener Handlungen des Agenten verstanden, die der Prinzipal nur kaum oder nur unter Inkaufnahme hoher Kosten kontrollieren kann (JOST 2001: 25). Die Gefahr, dass der Agent dies zu seinem eigenen Vorteil ausnutzt, wird als moral hazard bezeichnet. Als hidden information werden Informationsasymmetrien bezeichnet, die den Inhalt der Vertragsdurchführung betrifft. Das heißt, der Agent hat zusätzliche Informationen, die ihn den Erfolg der Aufgabendurchführung besser einschätzen lässt.

Die dritte Form von Informationsasymmetrien betrifft die Eigenschaften des Agenten. Für den Prinzipal kann es ex ante schwierig sein, sicher festzustellen, welche Eigenschaften, die bedeutend für die Durchführung der Arbeit sind, der Agent hat. Der Agent hingegen ist viel besser über diese Charakteristiken informiert (hidden characteristics). Dies kann zur so genannten adverse selection führen. In diesem Fall hat der Prinzipal den falschen Agenten für die erfolgreiche Durchführung der Arbeit gewählt. Bei Neueinstellungen versucht man als Reaktion auf die Gefahr der adversen Selektion verschiedene Formen von Arbeitsverträgen, z.B. mit unterschiedlichen Lohnhöhen, anzubieten oder Stellenanzeigen so zu gestalten, dass möglichst nur geeignete Agenten zur Einstellung kommen. Diese Verfahren werden als screening bezeichnet.

Die Prinzipal-Agenten-Theorie lässt sich in zwei wissenschaftliche Strömungen unterteilen. Man unterscheidet die normative (oder quantitativ-modelltheoretische Variante) und die positive Prinzipal-Agenten-Theorie (qualitativ-empirische Variante) (JENSEN UND MECKLING 1976, ELSCHEN 1991).

Die positive Prinzipal-Agenten-Theorie beschäftigt sich mit der Beschreibung und Interpretation der institutionellen Gestaltung von Auftragsbeziehungen. Sie erklärt zum Beispiel, warum unterschiedliche Vertragsgestaltungen in der Praxis vorkommen und wie sich Agenten gegenüber Prinzipalen innerhalb bestimmter institutioneller Rahmenbedingungen verhalten (FRANKE 1993: 38). Sie erlaubt Tendenzaussagen 
hinsichtlich der optimalen Vertragsgestaltung von Anreizsystemen. Neben einigen Veröffentlichungen, die der positiven Prinzipal-Agent-Theorie zuzuordnen sind (JENSEN UND MECKLING 1976), dominieren stark mathematisch geprägte Lösungen, die sich auf die normative Prinzipal-Agenten-Theorie stützen. Die normative PrinzipalAgenten-Theorie stellt der ökonomischen Forschung ein analytisches Instrument zur Verfügung, das durch mathematische Beweise und logische Herleitungen Empfehlungen zur Gestaltung von Vertragsbedingungen ableitet.

\section{Annahmen der normativen Prinzipal-Agenten-Theorie}

Das Grundmodell der normativen Prinzipal-Agenten-Theorie enthält einige grundlegende Annahmen, auf denen die mathematische Lösung basiert. Nachfolgend werden die wesentlichen Grundannahmen vorgestellt (LAUX UND LIERMANN 2003: 526 ff., LAUX 1988: 24 f., JOST 2001: 17 ff.)

1) Die Prinzipal-Agenten-Theorie geht von einem einperiodischen Handlungsspielraum aus.

2) Der Agent kann aus mehreren Handlungsalternativen eine Alternative auswählen. Der mit den Handlungsalternativen verbundene Erfolg hängt von einem unsicheren Umweltzustand ab. Es liegt folglich eine Entscheidungssituation unter Risiko vor.

3) Prinzipal und Agent lösen die Entscheidungssituation rational, d.h. unter Maximierung des Erwartungswertes ihres jeweiligen Nutzens (Bernoulli-Prinzip ${ }^{7}$ ).

4) Der Agent realisiert die Handlungsalternative, die seinen individuellen Erwartungsnutzen maximiert. Der Prinzipal wählt die Entlohnungsfunktion, die seinen Erwartungsnutzen maximiert und bezieht dabei in seine Überlegungen das individuelle Nutzenmaximierungsprinzip des Agenten mit ein.

5) Der Agent empfindet Arbeitsleid. Sein Erwartungsnutzen ist eine monoton fallende Funktion des Arbeitsaufwandes. Der Arbeitaufwand ist seine zweite Zielgröße in der Nutzenfunktion. Die erste Zielgröße ist die finanzielle Belohnung. Der Arbeitsaufwand

\footnotetext{
${ }^{7}$ Das „Bernoulli-Prinzip“ wird auch synonym als „Erwartungsnutzen-Prinzip“ oder „von-NeumannMorgenstern-Nutzen“ bezeichnet. Diesem Prinzip zufolge existiert für den Entscheidungsträger eine monoton steigende Risikonutzenfunktion $u(x)$ auf der Menge der Ergebnisse $x$. Die Handlungsalternativen können anhand des Erwartungswertes der Nutzen in eine Rangfolge gebracht werden. Die Alternative mit dem größten Erwartungsnutzen wird gewählt. Der Erwartungsnutzentheorie liegt ein kardinaler Nutzenbegriff zugrunde (BRANDES UND ODENING 1992).
} 
des Agenten kann mit Hilfe eines Skalars angegeben werden. Dieses bezeichnet man als Aktivitätsniveau. Es ist kardinal messbar und kann z.B. folgende Merkmale haben:

- Arbeitszeit,

- Arbeitsgeschwindigkeit,

- Gründlichkeit.

6) Der Agent akzeptiert eine Entlohnungsfunktion nur dann, wenn er einen Erwartungsnutzen von $\bar{u}$ erreichen kann. Dieser ist exogen vorgegeben. Es ist das Nutzenniveau, das der Agent bei alternativer Beschäftigung erreichen kann und als Reservationsnutzen bezeichnet wird. Es entspricht dem Nettonutzen aus der Arbeit für den Prinzipal. Diese Grundannahme wird als so genannte Teilnahmebedingung oder auch participation constraint bezeichnet.

7) Der Prinzipal ist indifferent zwischen den Handlungsalternativen. Der Nutzen des Prinzipals ist allein abhängig vom ökonomischen Erfolg nach Abzug der Belohnung (Nettoerfolg).

8) Als Bemessungsgrundlage werden nur solche Variablen berücksichtigt, die von beiden Parteien beobachtet werden können. Der Prinzipal kann ex post den Erfolg überprüfen, allerdings nicht den eingetretenen Umweltzustand und die realisierte Handlungsalternative.

9) Mit zunehmendem Aktivitätsniveau wird eine immer „günstigere“ Wahrscheinlichkeitsverteilung über den Erfolg erzielt.

10) Der Prinzipal kennt die Nutzenfunktion des Entscheidungsträgers sowie dessen Wahrscheinlichkeitsurteil hinsichtlich der denkbaren Umweltzustände.

11) Der Agent bezieht außerhalb der betrachteten Kooperationsbeziehung keine ungewissen Einkünfte.

12) Der Prinzipal ist risikoneutral oder schwach risikoavers.

Bestimmung eines optimalen Entlohnungsschemas mit Hilfe eines PA-Modells Im Folgenden soll ein optimales Entlohnungsschema für den Fall einer Arbeitsdelegation dargestellt werden (vgl. ODENING 1994). 
Gemäß Annahme 2) ist es dem Agenten möglich, zwischen verschiedenen Handlungsalternativen (hier vereinfacht nur zwei Alternativen)

$$
a_{i}, i=1,2
$$

$a_{i}=$ Handlungsalternativen des Agenten

zu wählen.

Der Output

$$
x_{i j}=x\left(a_{i}\right), j=1, \ldots, n
$$

$x_{i j}=$ monetärer und stochastischer Output

$$
\text { wobei } x_{1 j}<x_{2 j}
$$

des Arbeitseinsatzes ist monetär und stochastisch (ebenfalls Annahme 2).

In Abhängigkeit von den gewählten Handlungsalternativen/Aktionen $a_{i}$ des Agenten treten die bedingten Wahrscheinlichkeiten des Outputs $p_{i j}$ auf. Prinzipal und Agent haben dieselbe Einschätzung hinsichtlich der bedingten Wahrscheinlichkeiten.

Die Entlohnung des Agenten wird mit

$$
s_{j}=s\left(x_{i j}\right) \text { bezeichnet. }
$$

$s_{j}=$ Lohn des Agenten

Der Nettoerfolg (das Einkommen)

$$
\left(x_{i j}-s_{j}\right)
$$

$\left(x_{i j}-s_{j}\right)=$ Nettoerfo $\lg$ des Prinzipals

des risikoneutralen Prinzipals aus der Vertragsbeziehung ergibt sich als Residuum aus dem stochastischen Produktionsergebnis und dem Lohn des Agenten.

Der Nutzen des risikoaversen Agenten

$$
u_{j}=u\left(s_{j}\right)
$$

$u_{j}=$ Nutzen des Agenten

ist abhängig von seiner Entlohnung. 
Das Arbeitsleid des Agenten $c_{i}$ differiert zwischen den Handlungsalternativen. Bei Handlungsalternative 1 sei es kleiner als bei Handlungsalternative 2:

$$
c_{i}=c\left(a_{i}\right), c_{1}<c_{2}
$$

$c_{i}=$ Arbeitsleid des Agenten

Der Reservationsnutzen (Annahme 6) wird als $\bar{u}$ bezeichnet.

Ziel ist es, ein Entlohnungsschema $s$ zu finden, dass den Agenten die Alternative $\mathrm{a}_{2}$ wählen lässt. Es handelt sich um ein lineares Optimierungsproblem mit nicht-linearen Nebenbedingungen:

$$
\max \sum_{j=1}^{n}\left(x_{2 j}-s_{j}\right) p_{2 j}
$$

Maximiere den erwarteten Nettoerfolg/das Einkommen des Prinzipals unter den Nebenbedingungen:

$$
\sum_{j=1}^{n} u\left(s_{j}\right) p_{2 j}-c_{2} \geq \bar{u} \quad 1 . \text { Nebenbedingung (Teilnahmebedingung) }
$$

Die Differenz aus dem Erwartungsnutzen der Entlohnung und dem mit der Tätigkeit verbundenen Arbeitsleid (Nettonutzen des Agenten) muss größer sein als der Reservationsnutzen.

$$
\sum_{j=1}^{n} u\left(s_{j}\right) p_{2 j}-c_{2} \geq \sum_{j=1}^{n} u\left(s_{j}\right) p_{1 j}-c_{1} 2 . \text { Nebenbedingung (Anreizbedingung) }
$$

Damit der Agent die vom Prinzipal erwünschte Handlungsalternative 2 wählt, muss der Nettonutzen aus dieser Alternative größer sein als aus Alternative 1.

Zur Bestimmung des optimalen Entlohnungsschemas wird die folgende Lagrangefunktion gebildet:

(4) $L=\sum_{j=1}^{n}\left(x_{2 j}-s_{j}\right) p_{2 j}-\lambda\left(c_{2}+\bar{u}-\sum_{j=1}^{n} u\left(s_{j}\right) p_{2 j}\right)-\mu\left(c_{2}-c_{1}-\sum_{j=1}^{n} u\left(s_{j}\right)\left(p_{2 j}-p_{1 j}\right)\right)$ nach einigen Umformungen ergibt sich die folgende Gleichung: 
(5) $\frac{1}{u^{\prime}\left(s_{j}\right)}=\lambda+\mu\left(1-\frac{p_{1 j}}{p_{2 j}}\right)$

Diese kann Folgendermaßen interpretiert werden (ODENING 1994: 272): Ein optimales Entlohnungssystem setzt sich unter Berücksichtigung der oben genannten Annahmen aus einer erfolgsabhängigen und einer erfolgsunabhängigen Komponente zusammen. Dieses Ergebnis des P-A-Modells deckt sich mit vielen in der Praxis vorkommenden Empfehlungen zur Gestaltung von anreizkompatiblen Entlohnungssystemen. Eine grundlegende Annahme ist die Risikoaversion des Agenten. Wäre der Agent risikoneutral, dann sollte seine Entlohnung alleine auf einer erfolgsabhängigen Entlohnung basieren. Der risikoaverse Agent verlangt hingegen bei erfolgsabhängiger Entlohnung eine Risikoprämie.

\section{Anwendungsmöglichkeiten}

Die Prinzipal-Agenten-Theorie stellt der Betriebswirtschaft ein logisches Modell zur Lösung von Anreizsystemen zur Verfügung. Sie überzeugt durch ihre mathematisch präzise Lösung. Die Lösung des Standard-PA-Problems für Entlohnungssysteme in Form eines Grundgehalts mit einer leistungsabhängigen Entlohnungskomponente findet auch in der betrieblichen Praxis Anwendung, so dass auch empirisch nachweisbare Entlohnungssysteme durch die P-A-Theorie erklärt werden können.

\section{Problematische Verhaltensannahmen}

Kritisch zu sehen sind u.a. die Annahmen zum Risikoverhalten. So merkt RoUMASSET 1995: 161 ff.) in seinen Ausführungen zu Share-Cropping-Verträgen, die ein ähnliches Vertragsproblem wie die Beziehung von Arbeitgeber zu Arbeitnehmer darstellen (OTSUKA ET AL. 1992: 1968), an: “[...], there is little or no evidence [either] that tenants are substantially more risk averse than landlords [...] “.

Bei der Lösung komplexer Vertragsbeziehungen (z.B. bei mehrstufigen AgencyBeziehungen oder mehreren Agenten) gerät die normative Prinzipal-Agent-Theorie an ihre Grenzen und sollte durch andere Theorien erweitert werden (ELSCHEN 1991: 1011). Ein weiterer Kritikpunkt an der normativen Prinzipal-Agenten-Theorie ist die wenig empirische Ausrichtung. Die mathematisch hergeleiteten optimalen Vertragsgestaltungen weichen teils erheblich von den in der betrieblichen Praxis anzutreffenden Vertragsformen ab (MüLLER 1995: 62). Hauptkritiker der Prinzipal- 
Agenten-Theorie ist die verhaltenswissenschaftlich geprägte empirische Wirtschaftsforschung.

\subsubsection{Transaktionskostentheorie}

Die Transaktionskostentheorie als weiterer Forschungsansatz der Institutionenökonomik erklärt das Bestehen und die Vorzüglichkeit unterschiedlicher Organisationsformen, Märkte und Verträge mit den Kosten, die mit verschiedenen Transaktionen verbunden sind. Grundlegende Verhaltenseigenschaften der Akteure sind begrenzte Rationalität und Opportunismus (BECKMANN 2000: 47). Außerdem geht die Transaktionskostentheorie von der Risikoneutralität der Vertragspersonen aus und unterscheidet sich dadurch von der Prinzipal-Agenten-Theorie (ALLEN UND LUECK 2003: 10).

Die Höhe der Transaktionskosten wird von den Eigenschaften spezifische Investitionen, Unsicherheit, Häufigkeit und Messbarkeit bestimmt. Wie in Kapitel 2.1 deutlich wurde, ist der landwirtschaftliche Produktionsprozess besonders von Unsicherheit und schwieriger Messbarkeit der einzelnen Arbeitsschritte gekennzeichnet. Die Möglichkeit zu opportunistischen Verhalten und die Kontrollkosten, die notwendig sind, um dieses $\mathrm{zu}$ vermeiden sind entsprechend hoch. Je nach betrieblicher Ausgestaltung sind die Investitionen in das Humankapital als mehr oder weniger spezifisch anzusehen.

Da vor allem leitende Aufgaben kaum ausgelagert werden können, argumentieren transaktionskostentheoretisch geleitete Agrarökonomen, dass der landwirtschaftliche Familienbetrieb die vorzüglichere Organisationsform als der Lohnarbeitsbetrieb ist. Durch die Verknüpfung von einfachen, teils manuellen Arbeiten mit gleichzeitig leitenden Aufgaben ist es außerdem besonders in der Tierproduktion schwierig, Arbeitsabläufe in leicht kontrollierbare Aufgaben zu trennen, da unterschiedliche Anforderungsniveaus simultan ausgeführt werden (BECKMANN 2000: 174).

Abbildung 4 zeigt, wie sich die Transaktionskosten von Familien- und Lohnarbeitskräften mit der Anzahl der Beschäftigten verändern. 

ABBILDUNG 4: TRANSAKTIONSKOSTEN VON FAMILIEN- UND LOHNARBEITSKRÄFTEN BEI
GEMISCHT SPEZIFISCHEN INVESTITIONEN

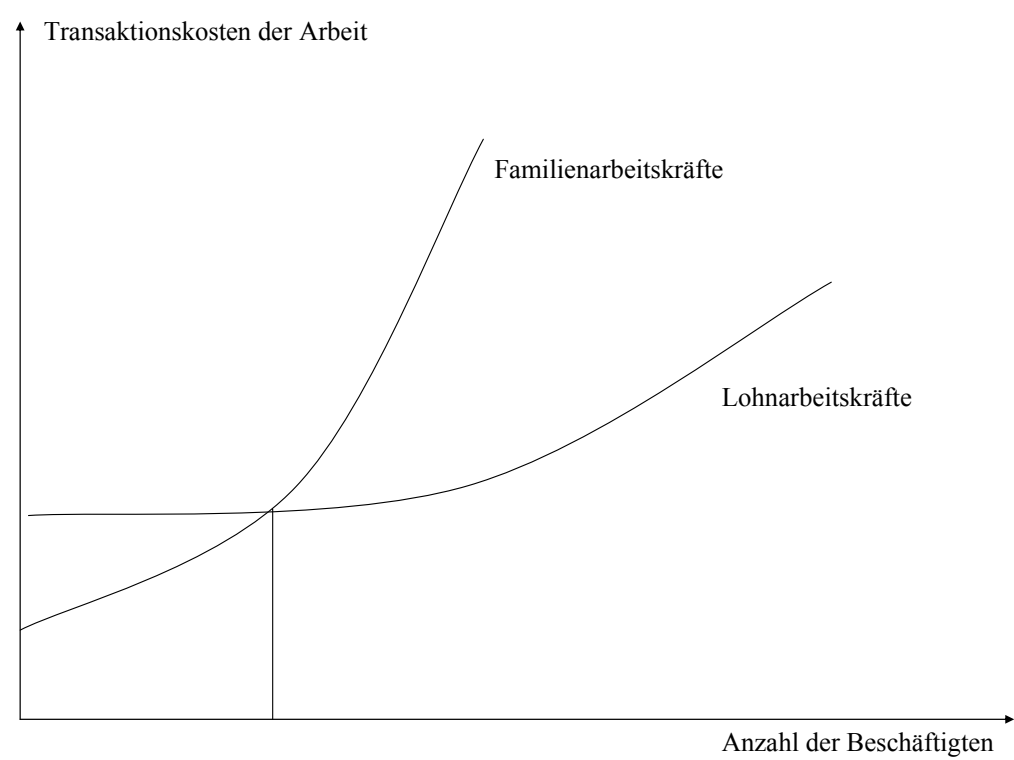

Quelle: BECKMANN 2000: 176

Bei wenigen Beschäftigten liegen die Transaktionskosten, die mit der Beschäftigung von Familienarbeitskräften verbunden sind, unter denen der Beschäftigung von Lohnarbeitskräften. $\mathrm{Ab}$ einer bestimmten Anzahl von Beschäftigten, die von BECKMANN (2000) jedoch nicht näher spezifiziert wird, liegen die Transaktionskosten der Beschäftigung von Lohnarbeitskräften unter denen der Familienarbeitskräfte. Dies erklärt sich daraus, dass wenige Familienarbeitskräfte auf Grund ihrer Verbundenheit zu Betrieb und Familie (vgl. Kapitel 2.1) intrinsisch motiviert sind, Lohnarbeitskräfte jedoch - nach Annahme der Transaktionskostentheoretiker - stärker motiviert werden müssen. Bei vielen Familienmitgliedern sinkt jedoch der Anreiz eines starken Engagements für den Betrieb, da sie aus dem Gewinn entlohnt würden und in einer großen Familie nur eine kleine Entlohnung zu erwarten hätten.

Aus dem Kurvenverlauf lässt sich ableiten, dass mit der Anzahl der Arbeitskräfte die Transaktionskosten steigen. Die Wahrscheinlichkeit opportunistischen oder Trittbrettfahrerverhaltens steigt mit der Anzahl der Beschäftigten, was eine leistungsabhängige Entlohnung als Sanktionsmittel attraktiver macht. Durch die steigenden Transaktionskosten, die sich vor allem durch einen zunehmenden Kontrollaufwand ausdrücken, wird der mögliche Erfolg eines leistungsabhängigen Anreizsystems gemindert. 
Für eine optimale Lohngestaltung ergeben sich aus der Transaktionskostentheorie die folgenden Schlussfolgerungen (BECKMANN 2000):

- Aufgaben in der Unternehmensführung lassen sich schwer messen. Eine leistungsorientierte Lohngestaltung würde hohe Transaktionskosten in Form von Kontrollkosten nach sich ziehen. Eine Bemessung der Arbeitsleistung an Inputgrößen (z.B. Arbeitszeit, Qualifikationsgrad, Dauer der Betriebszugehörigkeit) ist deshalb wahrscheinlich, da es schwierig ist, leicht messbare Outputparameter zu finden.

- In der Pflanzenproduktion gibt es einfache Arbeiten wie z.B. Erntearbeiten, die sich durch nicht ständige Lohnarbeitskräfte erledigen lassen. Die Möglichkeit zur Akkordentlohnung ist bei manchen Früchten möglich. Andere ausführende Arbeitsgänge in der Landwirtschaft sind jedoch schwer messbar, da sie durch stochastische Elemente beeinflusst werden. Für diese Arbeiten sind inputbasierte Entlohnungssysteme transaktionskostentheoretisch den outputbasierten überlegen.

- Auch in der Tierproduktion ist es schwierig, allgemein gültige Aussagen zu treffen, denn die Anforderungen und Tätigkeiten sind heterogen und reichen von einfachen manuellen (z.B. Ausmisten, Futterverteilen) zu hoch komplexen Aufgaben, die mit einem enormen spezifischen Fachwissen verbunden sind (z.B. Herdenmanagement, Brunstkontrolle, Gesundheitskontrolle). Hinzu kommt die Automatisierung von Arbeitsgängen mit elektronischer Überwachung. Bei einfachen oder automatisierten Arbeitsgängen können outputorientierte Entlohnungsformen vorteilhafter als inputorientierte Entlohnungsformen sein, da sie mit wenig Transaktionskosten belastet sind und einen Anreiz zu ,guter Arbeit“ bieten können. Leitende Tätigkeiten in der Tierproduktion sind jedoch schwierig zu messen. Es kann transaktionskostentheoretisch und auch praktisch vorteilhaft sein, Aufgaben, die mit besonders hohen spezifischen Investitionen verbunden sind, von außen zuzukaufen. Dies findet in der Praxis auch statt, zum Beispiel bei Auslagerung von Aufgaben an Dienstleister, wie beispielsweise Besamungstechniker.

Es bleibt festzuhalten, dass die Transaktionskostentheorie einen wichtigen Beitrag zur Erklärung verschiedener Organisationsformen und Verträge geleistet hat. Dies betrifft auch die agrarökonomische Forschung. Die Vorhersagen, die zur Zeit der deutsch- 
deutschen Wiedervereinigung zur optimalen Betriebsgröße landwirtschaftlicher Betriebe beruhend auf transaktionskostentheoretischen Überlegungen getroffen wurden, nämlich die Auflösung der Agrargenossenschaften zugunsten der Neugründung von Familienbetrieben, ist so jedoch nicht eingetroffen, wie Kapitel 2.2 zeigte. Das Ausmaß opportunistischen Verhaltens bei Lohnarbeitskräften ist entweder in der Realität nicht so groß wie von Transaktionskostentheoretikern angenommen, oder die Vorteile von Lohnarbeitsbetrieben hinsichtlich der Betriebsgröße überwiegen die systembedingten Nachteile. Auf die Frage der optimalen Betriebsgröße landwirtschaftlicher Betriebe soll hier jedoch nicht weiter eingegangen werden, da sie erschöpfend und kontrovers diskutiert wurde (vgl. z.B. SCHMITT 1995, BRANDES 1995, LANGBEHN 1994). Inwieweit die Aussagen zur Wahl der Entlohnungssysteme zutreffend sind, wird auch die empirische Analyse in Kapitel 5 zeigen.

\subsubsection{Die Theorie der Effizienzlöhne}

Unter dem Begriff „Personalökonomik“ versteht man die Anwendung mikroökonomischer Prinzipien auf Personalfragen (LAZEAR 2000a: F611). Personalökonomische Fragestellungen haben vorwiegend in den letzten 20 Jahren Eingang in ökonomische Publikationen gefunden.

Die Hauptgründe für die Bearbeitung personalwirtschaftlicher Fragestellungen durch Ökonomen liegen zum einen in der ökonomisch unbefriedigenden Bearbeitung durch Human-Resource-Spezialisten und zum anderen in der Verbreitung der Agency- und Kontrakttheorie, durch die die Ökonomen geeignete Konzepte zur Behandlung personalwirtschaftlicher Fragestellungen zur Verfügung haben (ebenda: F611).

Das Ziel der Personalökonomie ist es, ökonomische Instrumente anzuwenden, um die Handlungsweisen von Praktikern und Beratern a) $\mathrm{zu}$ verstehen und b) Handlungsempfehlungen zu geben (ebenda: F611). Die Personalökonomik nimmt die Rationalität der handelnden Akteure an. Die Herangehensweise lässt imperfekte Information und das Vorhandensein von Transaktionskosten $\mathrm{zu}$, jedoch geht sie davon aus, dass das Verhalten der Akteure von ihrer Interaktion bestimmt wird und nicht durch Kräfte außerhalb ihres Einflussbereiches. Effizienz ist ein zentrales Konzept der Personalökonomik. In der Personalökonomik werden alle Formen der Kompensation in monetärer Form ausgedrückt. Nicht-monetäre Formen der Kompensation werden durch ein Äquivalent ausgedrückt (ebenda: F613). 
Eine personalökonomische Lösung von Anreizproblemen bietet die Effizienzlohntheorie (STIGLITZ 2002: 464-465, SHAPIRO UND STIGLITZ 1984: 433 f.). Sie basiert auf der Beobachtung interindustrieller Lohnunterschiede. Demnach gibt es Unternehmen, die ihren Mitarbeitern überdurchschnittliche Löhne zahlen und diese über den überdurchschnittlichen Lohn an sich binden. Die Gewährung überdurchschnittlicher Löhne hat wirtschaftspolitische Auswirkungen, denn sie führen zu Arbeitslosigkeit, da zu dem überhöhten Preis mehr Menschen arbeiten wollen, als Angebot vorhanden ist. Informationsasymmetrien führen nach dem Modell von SHAPIRO UND STIGLITZ dazu, dass es nicht zur Markträumung kommt und Arbeitslosigkeit im Marktgleichgewicht vorkommt.

Abbildung 5 zeigt das Effizienzlohnmodell, das im Folgenden in seinen Grundzügen erklärt werden soll. Auf der Ordinate ist das Lohnniveau und auf der Abszisse die Menge an Arbeit abgetragen.

\section{ABBILDUNG 5: DAS EFFIZIENZLOHNMODELL VON SHAPIRO UND STIGLITZ}

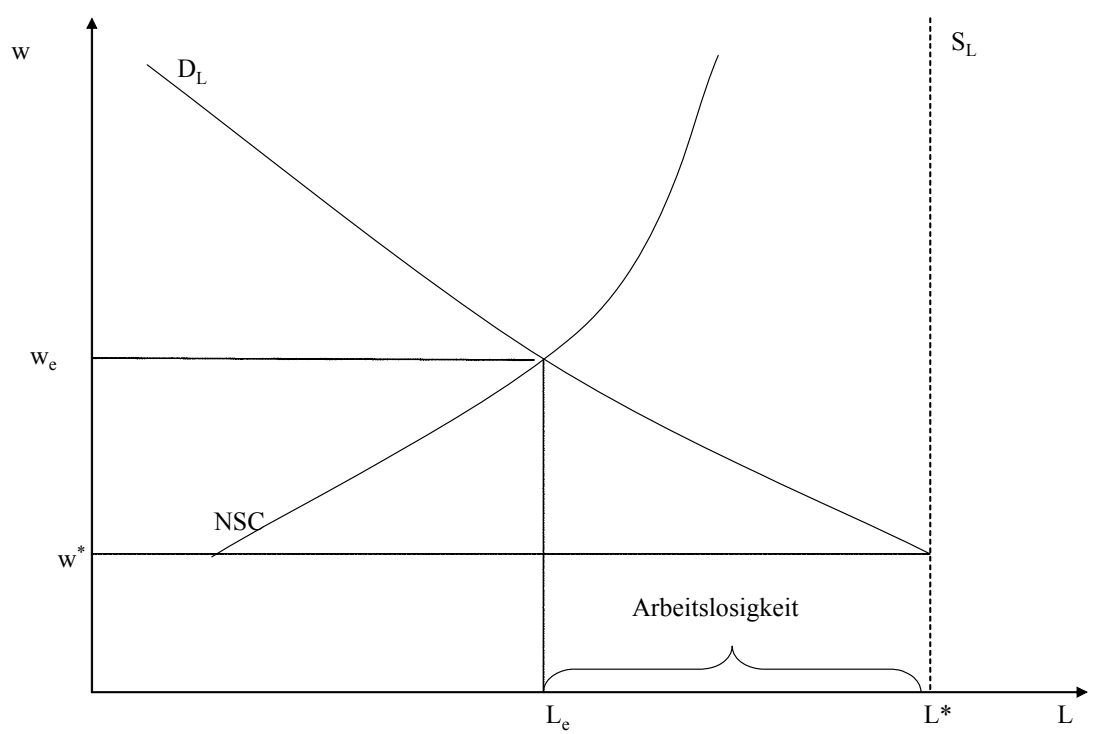

Legende: $\mathrm{w}=$ Lohn, $\mathrm{L}=$ Arbeitsmenge, $\mathrm{NSC}=$ No-Shirking-Constraint, $\mathrm{S}_{\mathrm{L}}=$ Arbeitsangebotskurve (aggregiert), $\mathrm{D}_{\mathrm{L}}=$ Nachfrage nach Arbeit (aggregiert)

Quelle: Eigene Abbildung nach SHAPIRO UND STIGLITZ 1984: 439

Mit $\mathrm{S}_{\mathrm{L}}$ wird die Angebotskurve an Arbeit bezeichnet, sie ist vollkommen unelastisch, da kurzfristig unabhängig vom Lohnsatz nur eine bestimmte Menge an Arbeit angeboten werden kann. Die aggregierte Nachfragekurve nach Arbeit ist mit $\mathrm{D}_{\mathrm{L}}$ gekennzeichnet. Die Effizienzlohnnebenbedingung, die im ursprünglichen Modell von SHAPIRO UND 
STIGLITZ als „No-Shirking-Constraint“ (NSC) bezeichnet wird, bewirkt, dass sich das Marktgleichgewicht nicht im Schnittpunkt von aggregierter Nachfrage- und Angebotskurve von Arbeit einfindet (mit dem zugehörigen Lohn $\mathrm{w}^{*}$ und der Arbeitsmenge $\mathrm{L}^{*}$ ), sondern im Schnittpunkt von Nachfragekurve und Effizienzlohnnebenbedingung mit dem höheren Lohnniveau $\mathrm{w}_{\mathrm{e}}$ und der verringerten Arbeitsmenge $\mathrm{L}_{\mathrm{e}}$. Die Effizienzlohnnebenbedingung ist eine steigende Kurve, da sie die kritische Lohnuntergrenze darstellt, unter der Arbeiter bummeln und diese mit dem Beschäftigungsniveau steigt.

Da bei L* Vollbeschäftigung herrschen würde, ist die Differenz zwischen $L^{*}$ und $L_{e}$ das Ausmaß der Arbeitslosigkeit. Die Abbildung macht deutlich, dass im Marktgleichgewicht ein gewisses Maß an Arbeitslosigkeit erzeugt wird.

Vier Erklärungsansätze existieren für die Gewährung überdurchschnittlicher Löhne als Anreizsystem zur Produktivitätssteigerung der Mitarbeiter (STIGLITZ 2002: 464 f.):

\section{Shirking-Modell}

Die klassische Erklärung für die Gewährung von Effizienzlöhnen ist das ShirkingModell. Durch die Zahlung überdurchschnittlicher Löhne steigen die Opportunitätskosten für das Faulenzen für den Mitarbeiter. Wenn der Mitarbeiter beim „Bummeln“ ertappt wird, riskiert er den Verlust des gut bezahlten Arbeitsplatzes. Das Unternehmen kann dadurch Kontrollkosten sparen.

\section{Turnover-Modell}

Das Turnover-Modell erklärt die Zahlung überdurchschnittlicher Löhne aus der Motivation, die Kosten, die mit häufigem Mitarbeiterwechsel in Verbindung stehen, gering zu halten. Diese Kosten beinhalten z.B. Investitionen in Weiterbildung, aber auch die Aufwendungen, die mit dem Anwerben neuer Mitarbeiter verbunden sind (STIGLITZ 1987: 21).

\section{Adverse-Selection-Modell}

Dieser Erklärungsansatz erklärt überdurchschnittliche Löhne mit dem Anziehen überdurchschnittlicher Mitarbeiter. Durch das vergleichsweise hohe Lohnniveau werden zwar unzureichend qualifizierte Personen nicht abgehalten, sich zu bewerben, es 
bewerben sich aber mehr hoch qualifizierte Personen als bei einer niedrigen Lohnofferte (STIGLitZ 1987: 24 ff., YELLEN 1984: 203).

Fair-Wage-Modell

Ein weiterer Erklärungsansatz für „efficiency wages“ ist die Lohngerechtigkeit. Dieser Ansatz geht auf die Publikationen von YELLEN und Co-Autoren zurück (YELLEN 1984, AKERLOF UND Yellen 1990). Demnach können durch hohe Löhne Produktivitätssteigerungen induziert werden, weil die Mitarbeiter den Lohn als „gerecht“ empfinden. Produktivitätssteigerungen sind nach diesem Ansatz durch moralische Effekte zu erklären.

Befragungen von Managern nach ihren Motiven, Effizienzlöhne einzusetzen bzw. Gehaltskürzungen zu vermeiden, sprechen eher für das Fair-Wage-Modell als für das Shirking-Modell. CAMPBELL UND KAMLANI 1997 (zitiert in GÄCHTER 2003: 45) konnten in einer Befragung zeigen, dass über $69 \%$ der Manager angaben, das Absenken von Löhnen würde dazu führen, dass die Mitarbeiter sich weniger loyal und dankbar ihrem Unternehmen gegenüber zeigen. Nur $4,4 \quad \%$ der befragten Personalverantwortlichen gaben an, die Mitarbeiter würden sich bei niedrigeren Löhnen weniger anstrengen, da sie einen Verlust der Arbeitsstelle weniger fürchteten. 26,4 \% der Befragten hielten beide Argumente für gleich wichtig.

Empirische Analysen zur Anwendung der Effizienzlohntheorie in der Landwirtschaft beschränken sich auf die Veröffentlichung von MORETTI UND PERLOFF (2002). Die empirische Analyse anhand eines Datensatzes der National Agricultural Worker Survey zeigt in einigen Punkten Konsistenz mit der Effizienzlohntheorie.

Effizienzlöhne können eine Alternative zu leistungsorientierter Entlohnungssysteme darstellen, wenn die Erfolgsbedingungen für eine solche Lohngestaltung nicht gegeben sind (MILGROM UND ROBERTS 1992: 253). So schlussfolgert HELLER in einem kritischen Beitrag zum Einsatz leistungsorientierter Entlohnung und zur Bedeutung finanzieller Anreize, die relativ geringen Löhne in der Landwirtschaft, würden dem Ruf der Agrarunternehmen schaden und böten vor allem jungen Nachwuchskräften wenig Anreiz für einen landwirtschaftlichen Werdegang. Außerdem würden niedrige Löhne demotivierend wirken und zu Fairnessdefiziten führen (HELLER 2003: 16 f.). 


\subsection{Ausgewählte Motivationstheorien}

Im folgenden Kapitel werden ausgewählte individualpsychologische Motivationstheorien dargestellt. Motivationstheorien lassen sich unterscheiden in Inhalts-, Prozess- und Aktionstheorien. Es werden einige Inhalts- und Prozesstheorien ausgewählt, deren Relevanz für die Arbeit als Auswahlkriterium entscheidend war.

\subsubsection{Bedürfnishierarchie nach Maslow}

Maslows Bedürfnishierarchie oder -pyramide (vgl. Abbildung 6) ist eine der bekanntesten Inhaltstheorien der Motivation (EISENFÜHR UND THEUVSEN 2004: 52). Obwohl sie empirisch nicht bewiesen ist (ebenda: 79), wird sie im Folgenden zum einen aufgrund ihrer Prominenz und zum anderen wegen ihrer möglichen Bedeutung für die Gestaltung von Anreizsystemen in der Landwirtschaft beschrieben.

Maslow geht davon aus, dass es eine festgelegte Reihenfolge von Bedürfnissen gibt, nach der diese für den Menschen verhaltensbeeinflussend sind. Für die Motivation eines Menschen ist immer nur die darüber liegende Ebene der Pyramide relevant.

Zunächst muss der Mensch physiologische Grundbedürfnisse wie z.B. Hunger und Durst stillen. Erst wenn diese Grundbedürfnisse befriedigt sind, strebt der Mensch nach Erfüllung des Sicherheitsbedürfnisses. $\mathrm{Zu}$ den Sicherheitsbedürfnissen zählen Sicherheit, Schutz, Angstfreiheit, Stabilität, aber auch das Bedürfnis nach Struktur, Ordnung, Gesetz und Grenzen (MASLOW 1996: 62 ff.). Es folgen das Bedürfnis nach Liebe, Freundschaft und Zugehörigkeit. Des Weiteren ist dem Menschen die Anerkennung durch andere und sich selbst ein Bedürfnis. Diese ersten vier Bedürfnisse der Pyramide werden als Defizitmotive bezeichnet, sind sie nicht befriedigt, wird dies als Defizit wahrgenommen. Eine Sättigung an Defizitbedürfnissen führt zu deren Bedeutungslosigkeit für das menschliche Verhalten. Das Bedürfnis nach Selbstverwirklichung (Entwicklungsmotiv) ist jedoch theoretisch unersättlich (EISENFÜHR UND THEUVSEN 2004: 52). 
ABBILDUNG 6: MASLOWS BEDÜRFNISPYRAMIDE

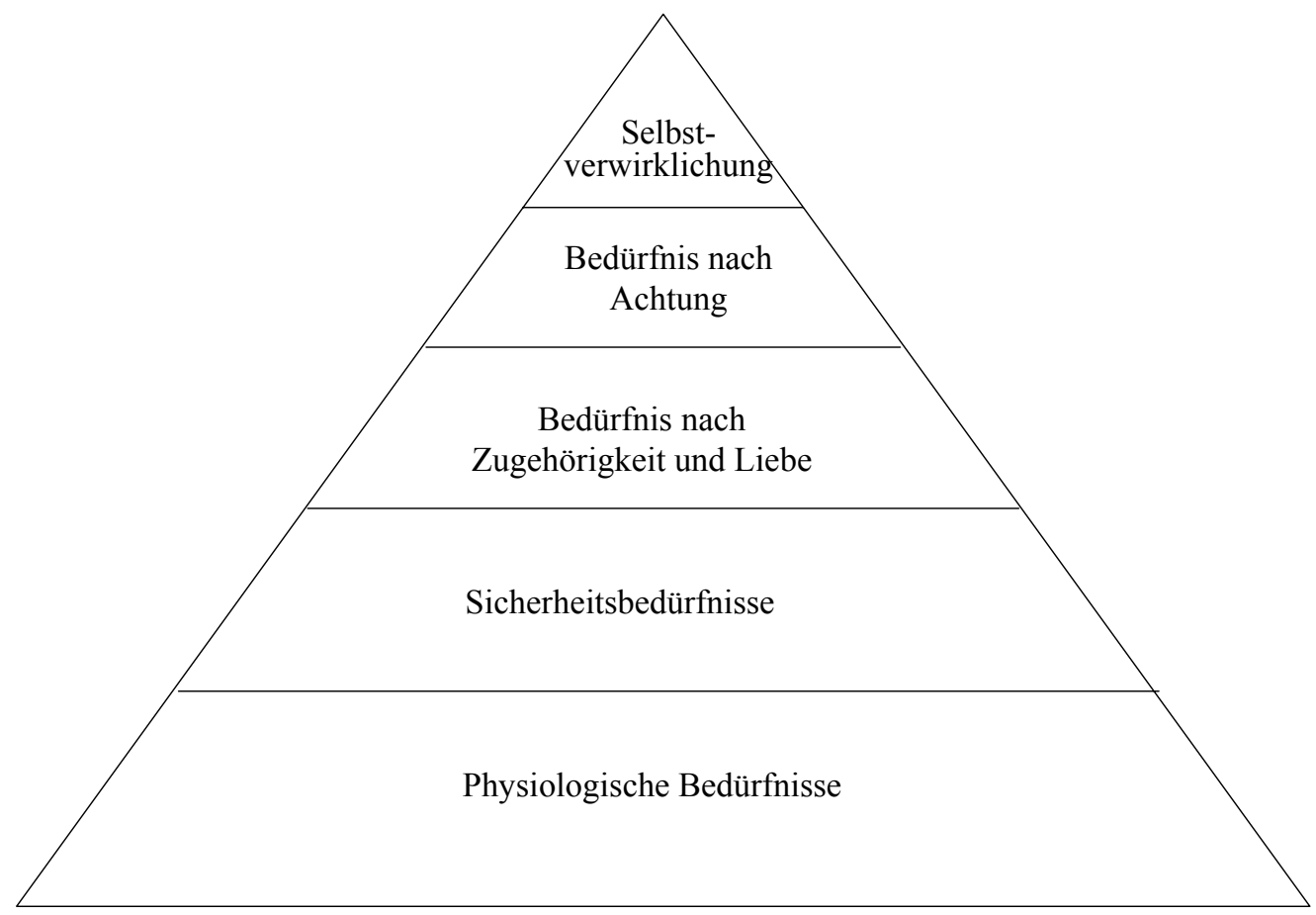

Quelle: Eigene Abbildung nach MASLOw 1996

Es stellt sich für die Anwendung von Anreizsystemen in der Landwirtschaft theoretisch die Frage, wie bedeutsam Anreize wie Leistungslöhne sein können, wenn existentielle Grundbedürfnisse wie das Streben nach einem sicheren Arbeitsplatz in der heutigen wirtschaftlichen Situation nicht befriedigt sind. Auf den Stellenwert eines sicheren Arbeitsplatzes wird in Kapitel 5.3.5 eingegangen. In Kapitel 2.2.1 wurde deutlich, dass das Lohnniveau in der Landwirtschaft im Vergleich $\mathrm{zu}$ anderen Wirtschaftsbranchen relativ gering ist. Daraus folgt, dass das Entlohnungssystem das Bedürfnis nach Sicherung des Lebensunterhalts abdecken sollte. Bei Vergütungssystemen, die aus einem fixen Grundlohn und einem leistungs- oder erfolgsorientierten Anteil bestehen, sollte ein Betriebsleiter darauf achten, dass der fixe Lohnbestandteil das Sicherheitsbedürfnis abdeckt, wenn der variable Anteil einmal nicht gewährt wird. Zwar eröffnet der Einsatz leistungsorientierter Entgeltsysteme für die Mitarbeiter potentiell die Perspektive, ein zusätzliches Einkommen zu erwirtschaften, nicht jeder Mitarbeiter wird dies aber schaffen. Deshalb ist es notwendig, dass auch für die leistungsschwächeren Mitarbeiter der Grundlohn ausreicht, um den Lebensunterhalt zu bestreiten. 


\subsubsection{Herzbergs Zweifaktoren-Theorie}

Herzbergs Zweifaktoren-Theorie begründet sich auf die Annahme, dass Motivation von Mitarbeitern von innen heraus kommen muss, also nur intrinsische Motivation eine dauerhafte Verbesserung der Leistung bewirken kann. In Anlehnung an Maslows Bedürfnispyramide (vgl. Kapitel 3.2.1), geht Herzberg davon aus, dass nur Anreize, die das Bedürfnis des Menschen nach Selbstverwirklichung befriedigen, wirklich motivieren. Eine Motivation durch extrinsische Anreize, z.B. Flexibilisierung der Arbeitszeit, Lohngestaltung und Zusatzleistungen (fringe benefits) würden den Menschen „verführen“, anstatt die Motivation aus sich heraus zu fördern. Dies sei langfristig kontraproduktiv und kostspielig. Er kritisiert explizit die Ansätze der Industrieökonomik und der Human-Relations-Bewegung, die den Schlüssel der Motivation im Design des Arbeitplatzes (,work flow“) bzw. im positiven Miteinander sehen (HERZBERG $1987^{8}$ : 110 f.).

Herzbergs Theorie stützt sich auf zwölf verschiedene Untersuchungen, die überwiegend in Amerika durchgeführt wurden (ebenda: 120). Die befragten Personen sollten benennen, welche Faktoren im Zusammenhang mit ihrer Arbeit Gefühle von extremer Zufriedenheit und Unzufriedenheit herbeigeführt hätten. Das Balkendiagramm der Abbildung 7 zeigt die prozentualen Häufigkeiten nach denen ein Faktor in Verbindung mit positiven oder negativen Erlebnissen am Arbeitsplatz genannt wurde. Es wird deutlich, dass die Faktoren, die zu Zufriedenheit oder Unzufriedenheit der Mitarbeiter am Arbeitsplatz führen, sich deutlich unterscheiden.

Die Faktoren, die zu Unzufriedenheit führen, bezeichnet Herzberg als Hygienefaktoren. D.h., sie sind notwendig, damit die Menschen Grundbedürfnisse wie die Sicherung des Lebensunterhalts befriedigen können, führen jedoch darüber hinaus zu keiner weitergehenden Motivation. Zu den Hygienefaktoren gehören Firmenpolitik und -verwaltung, Betreuung bzw. Überwachung, die Beziehung zu Vorgesetzten und Kollegen, Arbeitsbedingungen, Gehalt, Status und Sicherheit.

Die Faktoren, die zu Zufriedenheit führen, bezeichnet Herzberg als Motivatoren. Sie spiegeln das Bedürfnis des Menschen nach Selbstverwirklichung und

\footnotetext{
${ }^{8}$ Die zitierte Quelle ist ein Wiederabdruck des Originalartikels aus dem Jahr 1968, der ebenfalls im Harvard Business Review erschien. Der Originalabdruck wird ergänzt durch abschließende Bemerkungen des Autors zu den in der Zwischenzeit hinzugewonnen Erkenntnissen.
} 
Entwicklungsmöglichkeiten bei der Arbeit wider. Hierzu gehören Erfolg, Anerkennung, die Arbeit selbst, Verantwortung, Beförderung und Entwicklung.

ABBILDUNG 7: FAKTOREN, DIE DIE HALTUNG ZUR ARBEIT BEEINFLUSSEN

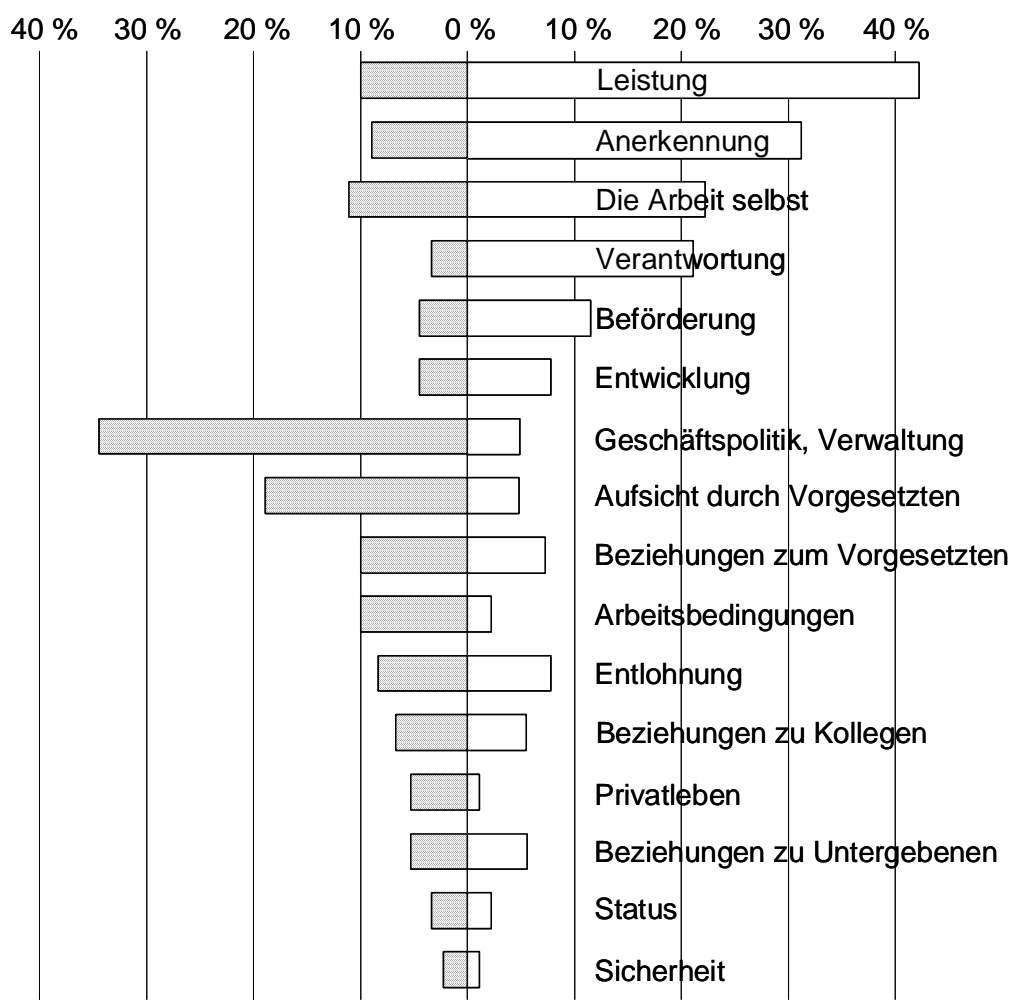

Quelle: EISENFÜHR UND THEUVSEN 2004: 53

Ein weiterer interessanter Aspekt an Herzbergs Theorie ist seine These, dass Zufriedenheit nicht das Gegenteil von Unzufriedenheit sei. Fehlt beispielsweise ein Motivator, so führe das nicht automatisch zu Unzufriedenheit, sondern eher zu NichtZufriedenheit. Die Anwesenheit eines Hygienefaktors schaffe wiederum nicht Zufriedenheit, sondern Nicht-Unzufriedenheit (ebenda: 112 f.).

Wie können Unternehmen nach Herzbergs Theorie Motivation ihrer Mitarbeiter herbeiführen? Herzberg empfiehlt ,job enrichment“, der Arbeitsplatz des Menschen muss mit Verantwortung angefüllt werden. Die folgende Tabelle 8 zeigt, mit welchen Maßnahmen dieser ,vertical job load“9 erreicht werden kann.

\footnotetext{
${ }^{9}$ Herzberg verwendet den Begriff des vertical job load als Gegensatz zum horizontal job load. Letzterer besteht seiner Meinung nach darin, Arbeitsplätze so umzustrukturieren, dass der Beitrag der einzelnen Mitarbeiter entweder reduziert wird oder weitere monotone Arbeiten dem alten Arbeitsplatz hinzugefügt werden.
} 
TABELLE 8: PRINZIPIEN DES „VERTICAL JOB LOADS“

\begin{tabular}{|l|l|}
\hline \multicolumn{1}{|c|}{ Prinzip } & \multicolumn{1}{|c|}{ Einbezogene Motivatoren } \\
\hline $\begin{array}{l}\text { (1) Einige Kontrollen wegnehmen, aber } \\
\text { zugleich Übernahme von Verantwortung für } \\
\text { Handlungen erhalten. }\end{array}$ & Verantwortung und persönliche Leistung \\
\hline $\begin{array}{l}\text { (2)Verantwortlichkeit jedes einzelnen für die } \\
\text { eigene Tätigkeit }\end{array}$ & $\begin{array}{l}\text { Verantwortung und Anerkennung der eigenen } \\
\text { Leistung }\end{array}$ \\
\hline $\begin{array}{l}\text { (3)Eigener Verantwortungsbereich für jeden } \\
\text { Mitarbeiter }\end{array}$ & $\begin{array}{l}\text { Verantwortung, persönliche Leistung und } \\
\text { Anerkennung }\end{array}$ \\
\hline $\begin{array}{l}\text { (4) Persönliche Freiheit und Weisungsbefugnis } \\
\text { der Mitarbeiter in ihrem Aufgabenbereich } \\
\text { gewähren. }\end{array}$ & $\begin{array}{l}\text { Verantwortung, persönliche Leistung und } \\
\text { Anerkennung }\end{array}$ \\
\hline $\begin{array}{l}\text { (5) Erstellen von periodischen Berichten für die } \\
\text { anderen Mitarbeiter }\end{array}$ & Interne Anerkennung \\
\hline $\begin{array}{l}\text { (6) Unbekannte und schwierigere Aufgaben } \\
\text { einführen. }\end{array}$ & Entwicklung und Lernen \\
\hline $\begin{array}{l}\text { (7) Zuteilung von speziellen oder spezifischen } \\
\text { Aufgaben an die Mitarbeiter, damit sie die } \\
\text { Möglichkeit haben Expertise zu erlangen. }\end{array}$ & Verantwortung, Entwicklung und Beförderung \\
\hline
\end{tabular}

Quelle: In Anlehnung an EISENFÜHR UND THEUVSEN 2004, HERZBERG 1987: 114

Neben den in der Tabelle aufgeführten Maßnahmen des ,job enrichments“ ist es nach Herzberg förderlich, wenn sich die Mitarbeiter als interne Kunden und Lieferanten einer bestimmten Kernaufgabe im Unternehmen ansehen. Wahrgenommenes ,job enrichment" nimmt demnach zu, wenn sich die Mitarbeiter der von ihnen erzeugten „Produkte“ und „Kunden“ gefühlsmäßig bewusst seien. Dadurch, dass die Mitarbeiter in einem gegenseitigen Austauschverhältnis zueinander stehen und Bericht erstatten, erfolgen Feedback und resultierender Lernprozess quasi automatisch (ebenda: 120).

Da Herzbergs Modell empirisch leicht prüfbar ist, wurde die Studie in mehreren Ländern wiederholt. Die Ergebnisse ähneln im Wesentlichen den ursprünglichen Untersuchungen.

Ein Kritikpunkt an Herzbergs Theorie ist die methodenbedingte Verzerrung der Ergebnisse. Die Mitarbeiter wurden erst nach den Faktoren, die Zufriedenheit oder Unzufriedenheit schufen befragt, bevor diese in das Theoriegerüst eingingen. Außerdem werden Schwächen in der Messmethodik und ein unwissenschaftlicher Umgang mit der Datenbasis moniert (SchOlZ 2000: 886, EISENFÜHR UND THEUVSEN 2004: 56 f.).

Für die Bedeutung leistungsorientierter Entlohnungssysteme prognostiziert das Modell von Herzberg die folgende Konsequenz: Da Entlohnung nur ein Hygienefaktor ist, ist es alleine notwendig, dass sie den notwendigen Lebensunterhalt deckt, damit der 
Mitarbeiter seine Grundbedürfnisse decken kann. Einen Antrieb zur Arbeitsmotivation würde dadurch nicht hervorgerufen werden.

Ausgehend von den in Tabelle 8 dargestellten Maßnahmen des vertikalen job loads ist $\mathrm{zu}$ untersuchen, wie diese in der landwirtschaftlichen Praxis umgesetzt werden könnten, um zu einem ,job enrichment“ nach Herzberg zu führen. Zunächst ist es möglich, den Mitarbeitern in gewissem Maße Verantwortung für die eigene Tätigkeit zu geben. Betrachtet man den Arbeitsgang „Aussaat“ im Pflanzenbau, so trägt ein Schlepperfahrer, der zum Beispiel die Aussaat vornimmt, eine relative hohe Verantwortung für seine Tätigkeit. Zwar wird wohl in vielen Fällen die Aussaatstärke durch den Betriebsleiter oder den Abteilungsleiter der Pflanzenproduktion vorgegeben, die Durchführung der eigentlichen Aussaat beeinflusst aber das Arbeitsergebnis stark. Dies trifft zum Beispiel die Saattiefe, das exakte Einsetzen der Drillmaschine und die Kontrolle der Särohre auf eventuelle Verstopfungen. Die Bedeutung dieser ausführenden Arbeiten kann durch Kommunikation und Anerkennung guter Arbeitsverrichtung klargestellt werden. Hiermit wären die Prinzipien 1 bis 3 und sogar das Prinzip 7 erfüllt, denn der betreffende Schlepperfahrer kann im Bereich Aussaat ein hohes Maß an Expertise erreichen, denn die Arbeit bietet an sich eine herausfordernde Aufgabe mit Möglichkeiten zur Perfektion in Abhängigkeit von der Erfahrung.

Die Übertragung von persönlicher Freiheit und Weisungsbefugnis (Prinzip 4) im Bereich „Aussaat“" kann jedoch nicht vollständig erfolgen, wenn sie Bereiche der Betriebleitung (Aussaatstärke, Sortenwahl) betreffen.

Die Erstellung von internen Berichten (Prinzip 5) in schriftlicher Form zwischen den Mitarbeitern scheint in der landwirtschaftlichen Praxis kaum vorstellbar. In mündlicher Form könnte dies jedoch gut möglich sein. Bleibt man bei dem oben genannten Beispiel, so kann der Mitarbeiter, der für die Aussaat zuständig ist, besondere Vorkommnisse den anderen Kollegen mitteilen.

Die Entwicklungsmöglichkeiten im landwirtschaftlichen Betrieb werden unter Umständen von der nächsten Hierarchieebene begrenzt, denn nur wenige landwirtschaftliche Lohnarbeitsbetriebe bieten auf Grund ihrer Größe oder Organisationsstruktur überhaupt Aufstiegsmöglichkeiten. Der fortschreitende technische Fortschritt impliziert jedoch schon lebenslanges Lernen im technischen Bereich. 


\subsubsection{Adams' Equity Theorie}

Die Equity Theorie von Adams ist den Prozesstheorien der Motivation zuzuordnen. Sie vereint zwei sozialpsychologische Konzepte und formuliert Konsequenzen aus diesen für das menschliche Handeln. Das erste Konzept ist das Konzept der relativen Deprivation und Belohnung, das auf STOUFFER und Mitarbeiter zurückgeht. Das zweite Konzept ist das Konzept der distributiven Gerechtigkeit, das von HOMANS formuliert wurde (ADAMS 1972: 134-135). Adams Leistung besteht darin, die beiden Konzepte zu einer Theorie zusammenzufassen und die Konsequenzen auf die Reaktion von Menschen, und vor allem von Mitarbeitern zu formulieren.

Das Phänomen relativer Deprivation geht auf zwei Veröffentlichungen im American Soldier (1949) zurück (STOUfFER ET AL. 1949). Die Autoren beschreiben, dass die Unzufriedenheit amerikanischer Soldaten mit Status und Beruf mit zunehmendem Bildungsgrad und Beförderungsmöglichkeiten steigt. Dieses Paradox erklärt sich dadurch, dass die höher gebildeten Soldaten eine höhere Erwartung an den Berufserfolg und -status haben als ihre schlechter gebildeten Kameraden. Sie fühlen sich im Vergleich $\mathrm{zu}$ den Karrieremöglichkeiten ihrer Referenzgruppe im zivilen Leben benachteiligt. Außerdem steigt die Unzufriedenheit mit der Wahrscheinlichkeit der Beförderung. Relative Deprivation bedeutet Enttäuschung von Erwartungen und führt zu einem Ungerechtigkeitsempfinden. Das Konstrukt der relativen Deprivation wurde in Experimenten bestätigt, in denen die Konsequenz auf das menschliche Handeln herausgearbeitet wurde. Menschen, die relative Deprivation erfahren, reagieren darauf mit Unzufriedenheit, Feindseligkeit gegenüber Personen, die den erwünschten Erfolg erreicht haben und Veränderungen in der Wahl ihrer sozialen Referenzgruppe (ADAMS 1972: 138).

Das Konzept der distributiven Gerechtigkeit geht über das Konzept relativer Deprivation hinaus. Es behandelt die Frage, wie Belohnungen gerecht unter Menschen, die sich in einem Austauschprozess befinden, verteilt werden sollten. Distributive Gerechtigkeit zwischen Personen ist dann hergestellt, wenn die Belohnungen (abzüglich der dadurch entstehenden Kosten), die sie in einem Austauschprozess erhalten, proportional zu ihren Investitionen sind. Als Investitionen werden alle relevanten Attribute im Austauschprozess angesehen, z.B. Alter, Geschlecht, Ausbildung, Zugehörigkeit zu einer ethnischen Gruppe, persönliche Fähigkeiten und Erfahrung. 
An einem Arbeitsplatz befinden sich in der Regel mehr als zwei Personen in einen gegenseitigen Austauschprozess. Jeder Mitarbeiter (z.B. eines Teams) erhält Belohnungen von Arbeitgeber und erwartet, dass diese in einem gerechten Verhältnis zu den anderen Mitarbeitern verteilt wird. Da die Wahrnehmung von gerechter Entlohnung im Verhältnis zur eingesetzten Investition auf der subjektiven Einschätzung der beteiligten Personen beruht, führt dass für den Arbeitgeber $\mathrm{zu}$ einer schwierigen Entscheidungssituation, die kaum optimal zu lösen ist. Hinzukommt, dass die Beteiligten das zur Bemessung der individuellen Investition maßgebliche Attribut möglicherweise unterschiedlich einschätzen. Der Wert von Ausbildung oder Erfahrung könnte beispielsweise unterschiedlich eingeordnet werden (ebenda: 141).

Das Konzept der distributiven Gerechtigkeit trifft wenig Aussagen darüber, wie Menschen reagieren, die die Gewinner eines ungleichen Austauschprozesses sind. Theoretisch könnten sich diese schuldig gegenüber den Verlierern fühlen. Es wird angenommen, dass Gewinner später als Verlierer auf Ungerechtigkeiten im Verteilungsprozess reagieren (ebenda: 141).

Nach ADAMS ist Gleichheit zwischen den Personen $p$ und $a$ hergestellt, wenn:

$$
\frac{O_{p}}{I_{p}}=\frac{O_{a}}{I_{a}}
$$

$O=$ Outcome, $I=$ Input,$p=$ Person,$a=$ Vergleichsperson

Mit $p$ wird die Person bezeichnet für die Gleichheit oder Ungleichheit besteht und deren Reaktionsmöglichkeiten auf Ungleichbehandlung später erläutert werden soll. Die Person mit der sich $p$ vergleicht, wird mit $a$ bezeichnet. Normalerweise sind $p$ und $a$ unterschiedliche Personen, die sich entweder in einem direkten Austauschprozess befinden oder sich in ihrer Beziehung zu einer dritten Person (z.B. einem Arbeitgeber) vergleichen. Es ist jedoch auch möglich, dass $a$ und $p$ dieselbe Person sind. Dieser Fall trifft $\mathrm{zu}$, wenn sich $p$ mit sich selbst in verschiedenen Situationen, z.B. in einem zweiten Arbeitsverhältnis oder mit vorhergehenden Arbeitsplätzen vergleicht (ebenda: 148). 
Die Ergebnisse, die die Personen $p$ und $a$ im Austauschprozess erzielen, werden mit $O$ (für Outcomes) bezeichnet. ADAMS beschränkt sich nicht auf materielle Belohnungen, sondern auch immaterielle Belohnungen wie Arbeitszufriedenheit können Ergebnis des Austauschprozesses sein. Bezug nehmend auf Herzbergs ZweifaktorenTheorie kann das Resultat der Anstrengungen auch ein negatives Vorzeichen haben, z.B. Unzufriedenheit am Arbeitsplatz. Als $I$ werden die so genannten Inputfaktoren der Personen $p$ und $a$ bezeichnet. Es sind Persönlichkeits- oder Leistungsattribute, die die Personen in den Austauschprozess einbringen (ebenda: 144-147). Notwendige Eigenschaften von In-und Outputfaktoren sind Relevanz und Anerkennung. Sie müssen von mindestens der Person, die sie einbringt oder erhält, als In- und Output erkannt werden und auch als relevant für den Austauschprozess angesehen werden. Probleme im Arbeitsprozess treten häufig dann auf, wenn die Personen sich nicht einig darüber sind, welches die relevanten Eigenschaften sind.

Für die Situation der Ungleichheit, gilt entweder:

$$
\begin{gathered}
\frac{O_{p}}{I_{p}}>\frac{O_{a}}{I_{a}} \text {, d.h. die Person } p \text { ist besser gestellt als die Vergleichsperson } a \text { oder } \\
\frac{O_{p}}{I_{p}}<\frac{O_{a}}{I_{a}} \text {, die Person } p \text { ist schlechter gestellt als die Vergleichsperson } a .
\end{gathered}
$$

Menschen haben verschiedene Möglichkeiten zu reagieren, wenn sie das Gefühl haben, nicht gleich wie ihre Referenzperson bzw. - gruppe $^{10}$ behandelt zu werden (ADAMS 1972: 151 ff.). Adams verfolgt hier das Konstrukt der kognitiven Dissonanz (FESTINGER 1957, zitiert in ADAMS 1972). Menschen die Ungleichbehandlung erfahren, empfinden dies als kognitive Dissonanz, die es durch folgende Möglichkeiten abzubauen gilt:

1) Um gleiche Behandlung zu einer anderen Person herzustellen, kann die betreffende Person ihren Input verringern oder erhöhen, bis Gleichheit hergestellt ist. Jedoch ist zu bedenken, dass manche Inputattribute nicht verändert werden können, wie z.B. das Geschlecht oder Alter. Deshalb ist davon auszugehen, dass sich die Inputänderung vor allem in anpassbaren Attributen wie der Produktivität und Arbeitsqualität manifestiert.

\footnotetext{
${ }^{10}$ Nach ADAMS ist eine Referenzperson bzw. -gruppe mit der Person $p$ in mindestens einer Eigenschaft vergleichbar (ADAMS 1972: 148).
} 
Diese Inputs haben gegenüber den anderen oben genannten Eigenschaften den Vorteil, dass sie das Outcome der Gegenseite verändern, da sie direkten Einfluss auf die Produktivität haben und dadurch eine geringere Inputänderung notwendig ist, um Gleichheit herzustellen (ADAMS 1972: $151 \mathrm{f}$.).

2) Außerdem kann die Person versuchen, das Ergebnis der Arbeit zu verändern. In einer Situation wahrgenommener Benachteiligung, beispielsweise durch einen Lohn, der im Verhältnis zu den eingebrachten Inputs als unadäquat angesehen wird, kann dies durch das Fordern einer Lohnerhöhung geschehen (ebenda: 156 f.).

3) Die Person kann In- und Output psychologisch verändern (ebenda: 159 ff.). In einem automatisierten Produktionsablauf sind Arbeitseinsatz und -ausstoß kaum manipulierbar. Ändert die Person kognitiv die Relevanz eines Inputfaktors, zum Beispiel die Bedeutung des Alters, so kann Ungleichheit abgebaut werden. In Bezug auf den Output kann sich die benachteiligte Person bisher unerkannte Vorzüge der eigenen Arbeit klarmachen (z.B. Aufgabenvielfalt).

4) Eine drastische Maßnahme um Ungleichheit abzubauen, ist das Verlassen des Arbeitsplatzes (,leave the field“). Dies kann durch Kündigung, Versetzung oder Abwesenheit geschehen. Empirische Ergebnisse zeigen, dass die Abwesenheitsrate bei Menschen höher ist, die sich über ihr Lohnniveau beschweren (ebenda: 161).

5) Änderung von In- oder Output der Vergleichsperson. Dies kann durch Druck oder freiwillig geschehen, bis dass gleiche Austauschverhältnis wiederhergestellt ist (ebenda: 161-163).

6) Um wahrgenommene Benachteiligung abzubauen, können Personen auch die Referenzgruppe wechseln (ebenda: 163 f.). Dies ist am Arbeitsplatz nicht immer möglich. Außerdem geht ADAMS davon aus, dass die Wahl der Referenzperson eine sehr stabile Beziehung ist. Die Referenzperson wird gewissermaßen als Anker zur Orientierung angesehen. Aus diesem Grund wird nach seiner Einschätzung ein Wechsel der Referenzperson nur vorgenommen, wenn andere Möglichkeiten der Angleichung des Austauschverhältnisses nicht möglich sind. 
Adams Equity Theorie ist relativ gut empirisch überprüft (EISENFÜHR UND THEUVSEN 2004: 65). Die zwei Konzepte, auf die die Theorie aufbaut, das Prinzip der relativen Deprivation und das Konzept der distributiven Gerechtigkeit, wurden empirisch in zahlreichen Experimenten nachgewiesen, um sie als gültige Konstrukte zu validieren.

Ein Experiment, das Adams Equity Theorie bestätigt, ist das Korrekturlese-Experiment. Hierzu wurden Studenten eingestellt, deren Aufgabe es war, Schriftstücke Korrektur zu lesen. Die Studenten wurden in drei Gruppen eingeteilt. Einer Gruppe wurde mitgeteilt, sie seien für die Tätigkeit ausreichend qualifiziert und würden für die Korrekturarbeiten 30 Cent/Seite erhalten. Der zweiten Gruppe wurde mitgeteilt, sie seien unterqualifiziert, würden aber den gleichen Lohn erhalten wie die ausreichend qualifizierten Mitarbeiter. Eine dritte Gruppe wurde als unterqualifiziert eingestuft und erhielt deshalb einen reduzierten Stücklohn von 20 Cent/Seite. Die Annahmen von ADAMS bestätigten sich. Die unterqualifizierte (zweite) Studentengruppe zeigte eine höhere Produktivität als die Vergleichsgruppen, die sich in gründlicherer Arbeit ausdrückte. D.h., diese Gruppe versuchte die zu ihren Gunsten bestehende Ungleichheit durch eine Inputerhöhung in Form von sorgfältiger Arbeit auszugleichen (ADAMS 1972: 153 f.).

Kritik an Adams Equity Theorie bezieht sich vor allem auf die Wahl der Referenzgruppe. Es ist nicht klar festzustellen, wen die betreffende Person als Referenzperson auswählt. Schwierig wird es die Konsequenzen abzuschätzen, wenn das Individuum mehrere Vergleichspersonen hat und zugleich Über- und Unterbelohung wahrnimmt. Eine weitere Schwierigkeit ist die Gewichtung der relevanten In- und Outputfaktoren durch das Individuum (EISENFÜHR UND THEUVSEN 2004: 65 f.).

Für die leistungsorientierte Lohngestaltung hat Adams Equity Theorie die folgenden Implikationen: Eine wichtige Erkenntnis ist es, dass Menschen soziale Vergleiche vornehmen und je nach Ausgang des Vergleichs ihren Einsatz davon abhängig machen können. Vom betreffenden Mitarbeiter wahrgenommene Benachteilungen gegenüber einer Referenzgruppe oder -person im Betrieb - sofern er diese gegenüber dem Arbeitgeber artikuliert - könnten durch Kommunikation der Unterschiede bei den Inputs abgebaut werden. Liegt die Referenzperson außerhalb des Betriebes, so könnten bisher nicht wahrgenommene Outcomes der Arbeit, zum Beispiel ein gutes Betriebsklima oder Entscheidungsfreiheit kommuniziert werden. 
ADAMS zitiert eine Beobachtung, in der im Vergleich zu Kassierern besser qualifizierte Angestellte eines Supermarktes Einkaufstüten einpacken mussten. Starke soziale Unterschiede zwischen Vorgesetzten und Angestellten senkten die Gesamtproduktivität des Betriebs (ADAMS 1972: 155 f.). Dieses Beispiel lässt sich in der Landwirtschaft anwenden. Ein Volontärverwalter hat eine als Outcome angesehene Weisungsbefugnis gegenüber angestellten Schlepperfahrern. Letztere reagieren mit Inputänderung, um Gleichheit herzustellen, da nicht Qualifikation, ausgedrückt in einem hohen Bildungsgrad, sondern Erfahrung, die sich in technischer Versiertheit manifestiert, als der relevante Input angesehen wird.

\subsubsection{Das Modell von Porter und Lawler}

Das Motivationsmodell von PORTER UND LAWLER (1968) gehört zu den Prozesstheorien der Motivation. Ursprünglich war es das Ziel, ein Modell zu entwickeln, das den Zusammenhang zwischen der Arbeitshaltung von Managern und ihrer Leistung erklären kann. Der Fokus auf Manager rührte daher, dass zu der Zeit als das Modell formuliert wurde, schon einiges über den Zusammenhang von Arbeitshaltung und Leistung von Arbeitern untersucht worden war (z.B. durch Herzberg), die Manager als Blickpunkt jedoch bis dahin vernachlässigt waren.

Außerdem wollten PORTER UND LAWLER ein überprüfbares Theoriegerüst schaffen, das eine Synthese verschiedener Ansätze vereint, anstatt den existierenden Theorien einen weiteren Baustein hinzuzufügen: "Past experience indicates that the atheoretical approach frequently produces at best a number of small isolated findings that do not fit together in any meaningful pattern, and thus, such findings provide little basis upon which to extrapolate to other situations.“" (PORTER UND LAWLER 1968: 6).

PORTER UND LAWLER präferieren von den existierenden Motivationstheorien die Expectancy-Theorien der Motivation und hier in erster Linie die Erwartungs-ValenzTheorie von Vroom. Nach Vroom wird der Erfolg von Anreizsystemen von zwei wesentlichen Einflussgrößen bestimmt: Die Valenz sagt aus, welchen Wert ein bestimmter Anreiz für einen Mitarbeiter hat. Dieser kann sowohl negativer als auch positiver Ausprägung sein. Ist eine ausgelobte Belohnung für den betreffenden Menschen unattraktiv, so wird er die zur Erreichung notwendige Handlung nicht ausführen. Die Erwartung besagt, dass der Mitarbeiter die Anstrengung nur 
unternehmen wird, wenn er die subjektive Wahrscheinlichkeit als hoch einschätzt, das Ziel auch zu erreichen (EISENFÜHR UND THEUVSEN 2004: 66 f.).

Abbildung 8 zeigt schematisch das Motivationsmodell von PORTER UND LAWLER. Im Folgenden werden die Einflussgrößen des Modells beschrieben.

\section{ABBILDUNG 8: DAS MODELL VON PORTER UND LAWLER}

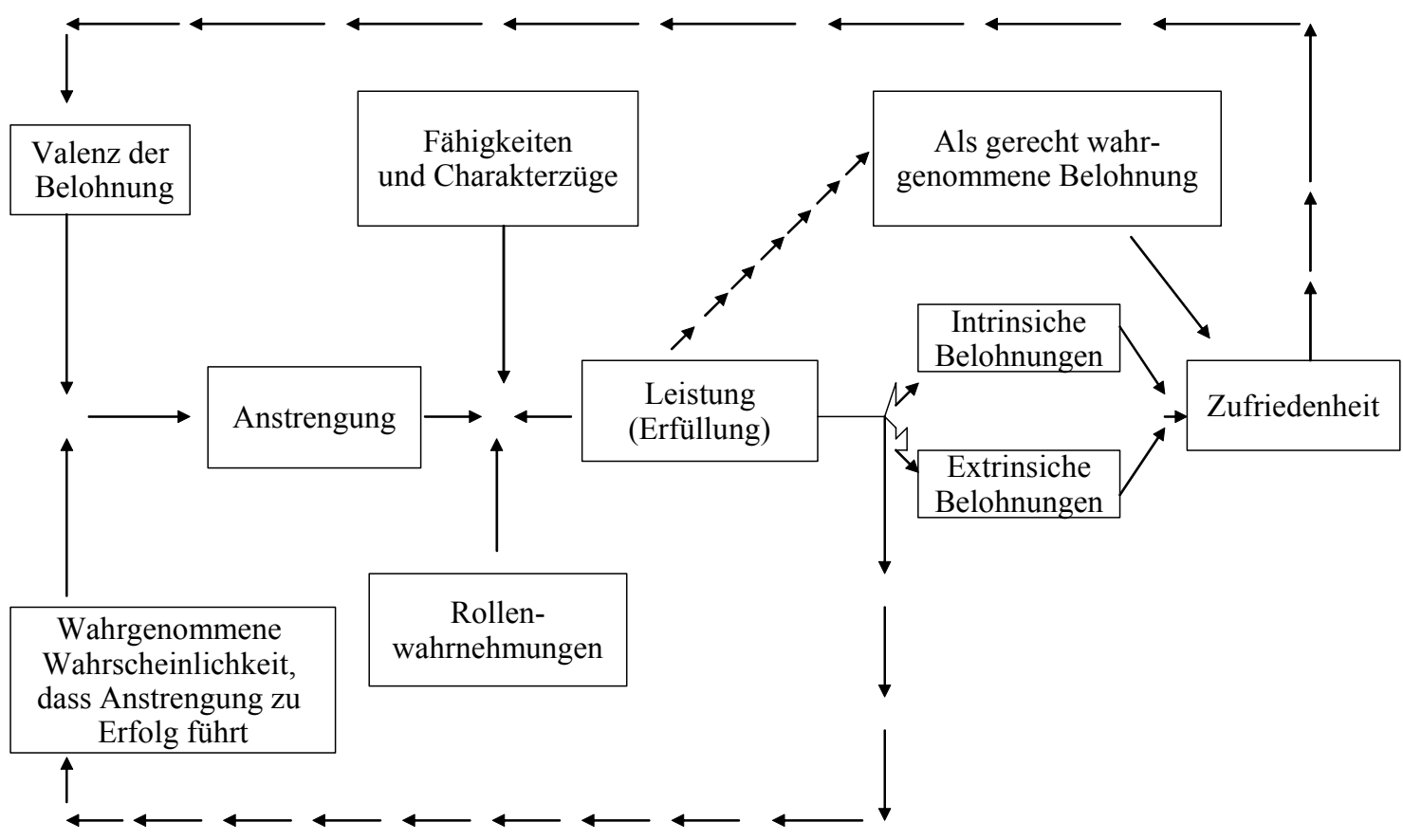

Quelle: In Anlehnung an PORTER UND LAWLER 1968: 17

\section{Valenz und Erwartung}

Analog zur Theorie von Vroom beeinflussen die Attraktivität der zu erwartenden Belohnung und die subjektive Wahrscheinlichkeit, dass mit der unternommenen Anstrengung auch die ausgelobte Belohnung erreicht werden kann, das Ausmaß der Anstrengung. Im Unterschied zu Vroom, der sowohl positive als auch negative Valenzen berücksichtigt, konzentrieren sich PORTER UND LAWLER (1968: 16) auf positive Valenzen. Die Bemessung der subjektiven Wahrscheinlichkeit über den Zusammenhang zwischen Anstrengung und Erfolg lässt sich nach Ansicht der beiden Autoren in zwei Komponenten aufspalten: Die erste Komponente ist die Wahrscheinlichkeit, dass Leistung von Anstrengung abhängig ist, und die zweite Komponente ist die Wahrscheinlichkeit, dass eine erwünschte Belohnung auf Leistung beruht. 
Anstrengung

Anstrengung oder Bemühung definieren PORTER UND LAWLER als die Energie, die für die Erfüllung einer Aufgabe aufgebracht wird. Sie korreliert nicht automatisch mit dem Erfolg dieser Anstrengung.

\section{Fähigkeiten und Charakterzüge}

Individuelle Fähigkeiten und Charakterzüge beeinflussen die Leistung und werden als langfristig relativ stabile Einflussgrößen angesehen. Sie stellen die obere Grenze der Leistungserfüllung dar, die durch Anstrengung überhaupt zu erreichen ist: „A person who has no musical abilities will never be a great pianist." (PORTER UND LAWLER 1968: 23).

\section{Rollenwahrnehmungen}

Für die Leistungserfüllung durch eine Person sind jedoch nicht alleine ihre Fähigkeiten von Bedeutung. Wichtig ist auch, welche Tätigkeiten und Bemühungen ein Individuum als bedeutend für die Leistung ansieht. Je nachdem, inwieweit sich diese mit denen der Organisation decken, wird das Leistungsniveau ausfallen.

\section{Leistung}

Die Variable Leistung bezieht sich darauf, wie gut eine Person ihren Job erfüllt. Den Begriff Leistung möchten PORTER UND LAWLER nicht auf den quantitativen Output oder die Produktivität der Arbeit beschränkt wissen. Zur Leistung zählt beispielsweise auch der freundliche Umgang mit Kollegen, wenn dieser notwendig für einen guten Arbeitsprozess ist oder Einfluss auf die Produktqualität hat. Mögliche Leistungsbestandteile, die von der Organisation nicht als relevant für die Erfüllung des Jobs angesehen werden, sind Teile des persönlichen Verhaltens. Leistung ist nach Definition der Autoren der Nettoeffekt der Anstrengung, die durch persönliche Fähigkeiten und Rollenwahrnehmung durch die betreffende Person beeinflusst wird (ebenda: 28). 


\section{Belohnungen}

Belohnungen sind gewünschte Resultate der Arbeit, die eine Person oder andere erhalten. Diese werden entweder von Dritten vergeben (extrinsische Belohnungen) oder die Person erfährt sie durch sich selbst (intrinsische Belohnung), zum Beispiel durch die Freude an der Erfüllung einer komplizierten Aufgabe. Ursprünglich hatten PORTER UND LAWLER in ihrem Modell nur die Variable Belohnung vorgesehen, die empirische Prüfung des Modells ergab jedoch, dass es im Hinblick auf ihre Beziehung zur Arbeitszufriedenheit wichtig ist, intrinsische von extrinsischen Belohnungen zu unterscheiden. Intrinsische Belohnungen und hier vor allem jene, die auf die Erfüllung von „Growth-Bedürfnissen“ wie Selbstverwirklichung oder Selbstständigkeit abzielen, werden durch Leistung bedingt, sofern es die Gestaltung des Arbeitsplatzes in Bezug auf die Entfaltungsmöglichkeiten des Individuums hergibt. Der Zusammenhang zwischen extrinsischen Anreizen und Leistung ist nach empirischen Ergebnissen der Autoren weniger deutlich.

Aufgrund seiner Komplexität ist es nicht einfach, das Modell von PORTER UND LAWLER in seiner Gänze empirisch nachzuweisen. Es formuliert aber sehr wichtige Erfolgsbedingungen leistungsorientierter Entlohnung und wird deshalb in Kapitel 3.4 wieder aufgegriffen. Für die landwirtschaftliche Praxis ist aus diesem Motivationsmodell die folgende Schlussfolgerung zu ziehen: Anreizsysteme sind immer dann wirksam, wenn die Belohnung von den Mitarbeitern geschätzt wird und sie die dafür notwendige Leistung auch mit einer hohen subjektiven Wahrscheinlichkeit erbringen können. Ob die Belohnung auch zu Zufriedenheit führt, hängt unter anderem davon ab, ob der Verteilungsprozess als gerecht wahrgenommen wird.

\subsection{Experimentelle Ökonomie und Mitarbeitermotivation}

Im folgenden Kapitel werden einige Ergebnisse der experimentellen Wirtschaftsforschung zur Mitarbeitermotivation dargestellt. Auch hier werden die Abgrenzungsschwierigkeiten gegenüber den zuvor dargestellten Ansätzen deutlich, denn die experimentelle Ökonomik hat auch Experimente zur Prinzipal-AgentenTheorie und zur Effizienzlohntheorie angestellt. Auch die Motivationstheoretiker haben sich den Experimenten zur Bestätigung ihrer Theorien bedient. Außerdem wurden einige der nachfolgend dargestellten Experimente nicht von Wirtschaftswissenschaftlern, sondern von Psychologen durchgeführt. 
Mikroökonomische Modelle gehen i. d. R. davon aus, dass sich der Mensch eigennützig und rational verhält. Die experimentelle Wirtschaftsforschung hat in Experimenten einige Verhaltensabweichungen festgestellt, die dem Rationalverhalten des homo oeconomicus entgegenstehen und dem Entscheidungsverhalten der Menschen gemäß der Erwartungsnutzen-Theorie entgegenstehen. Die daraus entwickelten Erklärungsansätze helfen vor allem bei unvollständigen Verträgen und Märkten mit nicht standardisierten Gütern (FEHR UND SCHMIDT 2001: 1). Arbeitsbeziehungen sind typischerweise unvollständige und implizite Verträge (MILGROM UND ROBERTS 1992: $353)$.

\subsubsection{Besitzeffekt, Verlustaversion und Status Quo Bias}

Der Endowment Effect (Besitzeffekt) ist ein in verschiedenen Experimenten nachgewiesenes, aus Perspektive des neoklassischen Menschenbildes anomales menschliches Verhalten. Die Zahlungsbereitschaft (willingness to pay, WTP) zum Kauf eines Gegenstandes fiel in Experimenten geringer aus als die Kompensationsforderung zum Verkauf des gleichen Gegenstandes (willingness to accept, WTA). Die Differenz zwischen WTP und WTA wird als Endowment Effect (Besitzeffekt) bezeichnet (KAHNEMAN UND KNETSCH 1992: 64 f., BRANDES ET AL. 1997: 463). Demnach schätzen Menschen einen Gegenstand mehr, wenn er ihnen bereits gehört als wenn sie ihn kaufen müssten.

Ein frühes Experiment zum Besitzeffekt wurde von KNETSCH UND SINDEN (1984) durchgeführt. Verschiedene Personen wurden entweder mit zwei Dollar oder einem Lotterielos ausgestattet. Nach einiger Zeit hatten die Personen die Möglichkeit zu handeln. Erstaunlicherweise kam ein Tausch zwischen Geld und Lotterielos kaum zustande, da die Menschen, die das Geld hatten, dieses lieber mochten als das Lotterielos und umgekehrt. Die Schlussfolgerung, dass Menschen lieb gewonnene Gegenstände ungern abgegeben, wurde damals von vielen Ökonomen mit dem Hinweis auf mögliche Lerneffekte der Versuchspersonen angezweifelt (KAHNEMAN ET AL. 1991: 194). Ein weiterer Kritikpunkt am Konzept des Besitzeffektes war in empirischen Ergebnissen begründet, die zeigten, dass die Diskrepanz zwischen Zahlungsbereitschaft und Kompensationsforderung zurückgeht, wenn die Versuchspersonen Erfahrungen in einem Marktumfeld sammeln konnten (ebenda: 194). Daraufhin führten KAHNEMAN ET AL. zwei weitere Versuchsreihen durch, die den Besitzeffekt bestätigen konnten. 
Wirtschaftsstudenten der Cornell-Universität wurden in einem ersten Marktexperiment mit „,induzierten Wertstücken“ (induced value token) ausgestattet, deren fiktiver individueller (induzierter) Wert den Personen, die sie erhielten, bekannt gegeben wurde. Handel (in mehreren Phasen) zwischen Studierenden, die Wertstücke erhalten hatten, und Personen, die keine erhalten hatten, führte $\mathrm{zu}$ einem Marktpreis, der zuvor prognostiziert worden war. Die induzierten Nachfrage- und Angebotskurven des Experimentes entsprachen den theoretisch errechneten Nachfrage- und Angebotskurven und bestätigten, dass die Studenten das Experiment verstanden hatten.

Im nächsten Schritt wurde ein Teil der Studierenden mit Kaffeetassen ausgestattet, die es im Buchladen der Universität für sechs Dollar zu kaufen gibt. Wie im vorangegangenen Experiment wurde der Versuch viermal durchgeführt, von denen jedoch nur einer nach dem Zufallsprinzip zählte. Eine fast identische Versuchsreihe wurde mit Rollminen-Kugelschreibern wiederholt, die mit einem Preis von \$3,98 gekennzeichnet waren. Die Verkäufer mussten ihre Gegenstände nicht verkaufen, wenn ihnen der angebotene Preis zu niedrig erschien. Abweichend vom ersten Experiment mit den induzierten Wertstücken wurde die Hälfte der theoretisch errechneten Gleichgewichtsmenge gehandelt. ${ }^{11 .}$ Der Medianpreis zu dem Personen bereit waren, Tasse bzw. Kugelschreiber zu verkaufen, lag in mehreren Versuchen ungefähr doppelt so hoch wie der Median-Kaufpreis (ebenda: 196).

Aufgrund der Angaben aus Versuchsreihen, bei denen den Kugelschreiberbesitzern verschiedene Kaufangebote unterbreitet wurden, die sie entweder annehmen oder ablehnen konnten, konnten für die verschiedenen Individuen Indifferenzkurven zwischen Geld und Kugelschreibern gezeichnet werden. Die Indifferenzkurven verlaufen unterschiedlich je nachdem, ob eine Person zuerst Geld oder Kugelschreiber hatte, und schneiden sich, was der mikroökonomischen Theorie widerspricht. KAHNEMAN ET AL. schlussfolgern auf Basis einer weiteren Studie, dass es nicht die Attraktivität des Gegenstandes ist, die dazuführt, dass er ungern abgegeben wird, sondern der mit der Abgabe verbundene Schmerz (ebenda: 197).

Eine dem Besitzeffekt verwandte Verhaltensabweichung vom Rationalprinzip ist der so genannte Status Quo Bias (SAMUELSON UND ZECKHAUSER 1988 zitiert in KAHNEMAN

\footnotetext{
${ }^{11}$ Unter der Annahme, dass Studenten, die zufällig eine Tasse oder einen Kugelschreiber erhielten, mit 50\% Wahrscheinlichkeit Tassenliebhaber oder - hasser (gleiches gilt für Kugelschreiber) sind.
} 
UND KNETSCH 1992: 68-70). Die ökonomische Bewertung von zwei Handlungsalternativen sollte eigentlich unabhängig davon sein, welche der beiden Handlungsoptionen den Status Quo darstellt. Tatsächlich neigen jedoch Menschen dazu, die Handlungsoption $\mathrm{zu}$ bevorzugen, die den Status Quo darstellt, sie sind strukturkonservativ. Die mit der Aufgabe des Status Quo verbundenen Nachteile scheinen stärker zu wirken als die möglichen Vorteile.

Die Orientierung von Menschen am Status Quo konnte in verschiedenen Versuchen bewiesen werden. Unter anderem wurden Versuchspersonen mit der Entscheidung konfrontiert, in welches Finanzportfolio sie ein fiktives Erbe investieren würden. Sie hatten die Auswahl zwischen unterschiedlich risikobehafteten Investitionen. Gab man den Personen vor, ein Teil des Erbes sei bisher in einer der alternativen Anlageformen investiert gewesen, so entscheiden sich mehr Menschen für diese Investitionsalternative. Das gleiche Versuchsdesign wurde in verschiedenen Szenarien wiederholt. SAMUELSON UND ZECKHAUSER schlussfolgern daraus, dass eine Alternative häufiger gewählt wird, wenn sie den Status Quo dargestellt. Außerdem steigt der Vorteil des Status Quo mit der Anzahl der zur Auswahl stehenden Alternativen (ebenda: 69).

Besitzeffekt und Orientierung am Status Quo lassen sich durch Verlustaversion erklären. Die Verlustaversion ist eine wichtige Implikation der Prospect Theory, einer Entscheidungstheorie für Entscheidungen unter Unsicherheit, die von Vernon Smith und Amos Tversky als Alternative zur von Neumann-Morgenstern'schen Entscheidungstheorie entwickelt wurde. Danach bewerten Menschen Verluste unterhalb eines neutralen Referenzpunktes stärker als mögliche Gewinne. Die Prospect Theory unterteilt den Entscheidungsprozeß von Menschen in zwei Phasen. In der ersten Phase („Editierungsphase“) wird das Entscheidungsproblem mental verarbeitet. In der „Evaluierungsphase“ wird verschiedenen Handlungsoptionen ein subjektiver Wert beigemessen. Die Alternative mit dem höchsten subjektiven Wert wird sodann als attraktivste Option ausgewählt (BOFINGER UND SCHMIDT 2003: 108).

Die folgende Abbildung 9 zeigt den Verlauf einer typischen Wertfunktion, die sich aus dem oben genannten Sachverhalt ergibt. 
ABBILDUNG 9: VERLAUF DER WERTFUNKTION

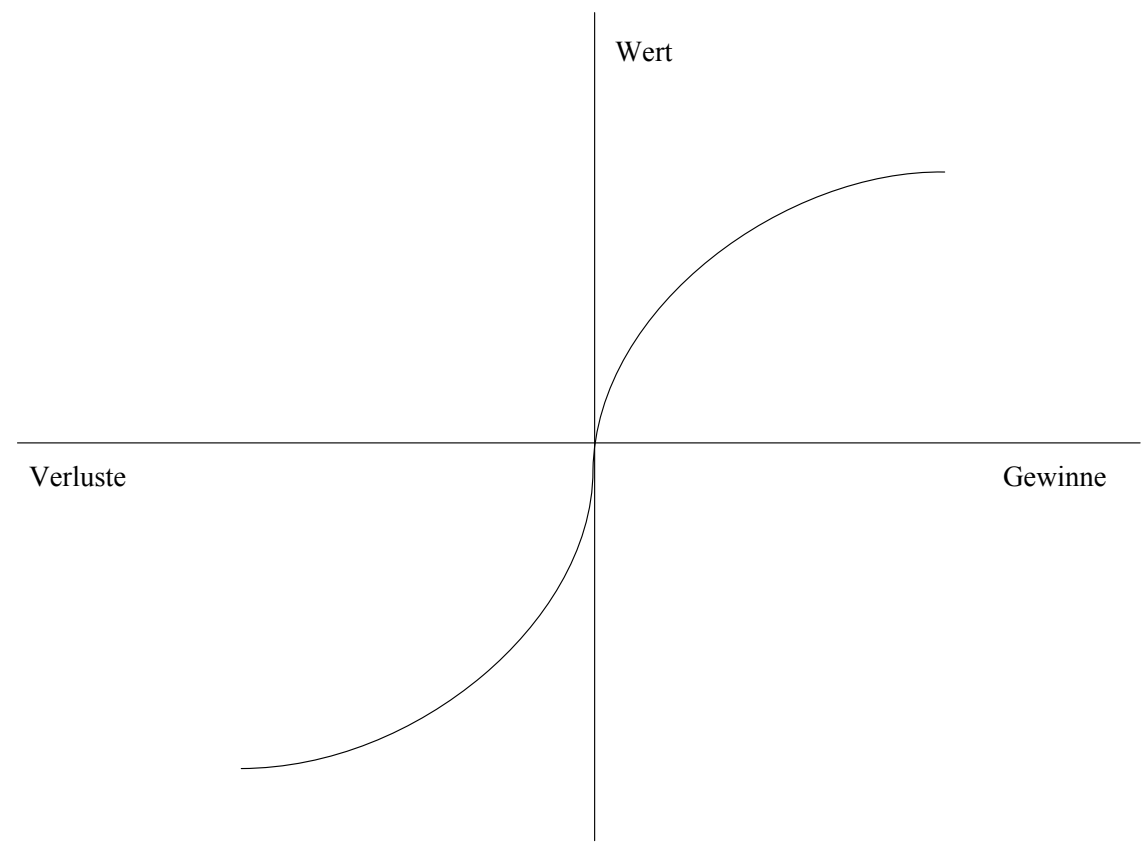

Quelle: KAHNEMAN ET AL. 1991: 200

Die Wertfunktion ist charakterisiert durch einen S-förmigen-Verlauf. Der Wert verschiedener Alternativen orientiert sich am Referenzpunkt, der psychologisch als Anker fungiert und sich im Ursprung des Koordinatensystems befindet. Auf der Abzisse sind die Gewinne bzw. Verluste im Vergleich zu einer Situation dargestellt, auf der Ordinate die Werte, die ein Individuum der jeweiligen Situation beimisst. Die Steigung der Wertfunktion verläuft im Gewinnbereich flacher als im Verlustbereich. Dadurch wird der Sachverhalt abgebildet, dass Individuen Verlusten eine höhere Bedeutung beimessen als Gewinnen in gleicher Höhe. Außerdem verdeutlicht der S-förmigeVerlauf, dass Menschen große Verluste bzw. Gewinne weniger stark bewerten als kleinere (ebenda: 109).

FEHR UND GOETTE (2005) konnten in einem Experiment mit Schweizer Fahrradkurieren, deren Entlohnung vollständig an den Umsatz der Auslieferung gebunden ist, zeigen, dass sich eine Lohnerhöhung auf die Anstrengung pro gefahrener Schicht negativ auswirkt. Dieses begründen die Autoren mit Verlustaversion, die bei einem Teil der Kuriere in einem Lotterieexperiment nachgewiesen werden konnte. Sie gehen davon aus, dass sich die Kuriere an ihrem Tageseinkommen als Referenzpunkt orientieren. Oberhalb des Referenzpunktes (Tageseinkommen vor Experimentdurchführung) ist der Anreiz sich anzustrengen durch die Lohnerhöhung geringer geworden, da das anvisierte Tageseinkommen schneller erreicht wird. 
Abschließend sei anzumerken, dass es Situationen gibt, in denen die Differenz zwischen WTP und WTA so groß ist, dass sie allein durch Verlustaversion nicht zu erklären ist. Moralische Gründe können der Erklärungsgrund für diese extremen Diskrepanzen sein. Dies ist zum Beispiel bei der Frage nach der Zahlungsbereitschaft für die Risikominimierung im Umgang mit giftigen Substanzen oder bei NIMBY ${ }^{12}$-Projekten der Fall (KAHNEMAN ET AL. 1991: 201 ff.).

Aus den oben dargestellten Verhaltensanomalien lassen sich folgende Schlussfolgerungen für die praktische Gestaltung von Anreizsystemen ableiten:

- Arbeitet ein Mitarbeiter bereits in einem bestimmten System im Unternehmen (z.B. Art der Entlohnung, Arbeitszeitregelung), so wird er eine Änderung des Systems ungern hinnehmen, auch wenn ihm im Austausch ein objektiv gleichwertiges (aber anders gestaltetes) System angeboten wird.

- Neue Lohn- und Beschäftigungssysteme lassen sich mit neuen Mitarbeitern leichter implementieren als mit älteren Mitarbeitern, da diese andere Referenzpunkte haben.

Dass diese Ergebnisse verhaltenswissenschaftlicher Ökonomik praktische Relevanz haben, zeigt der folgende Hinweis (SÄCHSISCHE LANDESANSTALT FÜR LANDWIRTSCHAFT 2002: 40) nachdem sich ,[...] einmal eingeführte Vergütungsregelungen oftmals schwer verändern lassen“. Änderungen an den eingeführten Vergütungsregelungen können leicht $\mathrm{zu}$ Widerständen und Unzufriedenheit bei den Mitarbeitern führen und eine demotivierende Wirkung hervorrufen (ebenda).

${ }^{12}$ NIMBY-Projekte (Not in my backyard) sind Projekte, die zwar gesellschaftlich notwendig sind, die man jedoch nicht vor der eigenen Haustür haben möchte. Ein Beispiel für NIMBY-Projekte ist die Allokation von Mülldeponien oder nuklearen Zwischen- und Endlagern (FREY ET AL. 2001: 578). 


\subsubsection{Verdrängungseffekt}

Für die Frage, welche Form des Entlohnungssystems - leistungsorientiert oder nicht leistungsorientiert - gewählt werden soll, ist ein Blick auf die Motivationswirkung verschiedener Systemen zu richten. Die Motivation von Menschen kann intrinsischer oder extrinsischer Natur sein. Intrinsisch motivierte Mitarbeiter erledigen die Arbeit um ihrer selbst willen (BECKER 1995: 37, WOLFF UND LAZEAR 2001: 13). Zum Beispiel auf Grund von Freude an der Tätigkeit, dem Wunsch nach Anerkennung der Leistung oder aus altruistischen Zielen (z.B. in der ehrenamtlichen Tätigkeit). Extrinsische Motivation zielt hingegen auf eine indirekte Nutzenerfüllung ab. Die Tätigkeit wird ausgeführt, um dadurch etwas anderes zu erreichen (z.B. mehr Freizeit, teure Hobbies).

Wie in Kapitel 3.1 dargelegt wurde, gehen neoklassische Forschungsansätze zur Motivation von Mitarbeitern von einer durchweg positiven Wirkung extrinsischer Anreize aus und klammern intrinsische Anreize aus den Betrachtungen weitgehend aus, da sie ökonomisch schwer operationalisierbar sind (WOLFF UND LAZEAR 2001: 25-26)

Leistungsorientierte Entlohnungssysteme zielen auf die Förderung extrinsischer Motivation ab. Die Motivationswirkung beruht auf dem Preiseffekt, d.h. auf den Opportunitätskosten, die mit „Bummeln“ bei der Arbeit verbunden sind. Ein Kritikpunkt an leistungsorientierten Entlohnungssystemen liegt im möglichen Auftreten eines Verdrängungseffekts (auch Crowding-Out-Effekt genannt). Experimente haben gezeigt, dass intrinsische Motivation durch extrinsische Motivation verdrängt werden kann (FREY ET AL. 2001: 563 ff., THEUVSEN 2003: 489).

Die Möglichkeit negativer Wechselwirkungen zwischen extrinsischer und intrinsischer Motivation basiert auf sozialpsychologischen Theorien. ${ }^{13}$ Eine unter ihnen, die in die ökonomische Theorie Eingang gefunden hat, ist die kognitive Bewertungstheorie von DECI (zitiert in FREY ET AL. 2001 oder auch THEUVSEN 2003). Kinder zeigen zum Beispiel weniger intrinsische Motivation ihren Eltern im Haushalt zu helfen, wenn sie dafür Geld erhalten, da dadurch Altruismus untergraben wird.

\footnotetext{
${ }^{13}$ Eine ausführliche Metanalyse zu Experimenten, die den Zusammenhang zwischen extrinsischer und intrinsischer Motivation untersuchen, findet sich in DECI ET AL. 1999.
} 
Der Verdrängungseffekt wurde von FREY und Co-Autoren in die ökonomische Theorie eingeführt. Zahlreiche Experimente zeigten, dass unter bestimmten Umständen der Verdrängungseffekt auftaucht. Der Preiseffekt extrinsischer Motivation wird dabei nicht in Frage gestellt. D.h., es wird grundsätzlich angenommen, dass die neoklassische Theorie greift und extrinsische Anreize isoliert betrachtet einen durchweg positiven Einfluss auf die Arbeitsmotivation haben. Zum Preiseffekt kommt jedoch der Verdrängungseffekt hinzu, also die Wirkung von extrinsischen Anreizen auf die intrinsische Motivation. Dieser kann in Abhängigkeit von der individuellen Situation positiv, neutral oder negativ wirken. Preis- und Verdrängungseffekt ergeben zusammen den Netto- oder Gesamteffekt, der die letztendliche Wirkung der extrinsischen Belohnung zeigt.

Der Gesamteffekt wird beeinflusst von der Höhe des (immer positiven) Preiseffekts und der Richtung des Verdrängungseffekts. Der Verdrängungseffekt wird von den folgenden Größen beeinflusst.

- Die Höhe der intrinsischen Motivation hat eine Auswirkung auf den Verdrängungseffekt. FREY geht davon aus, dass Arbeiter ohne Verantwortung und monotonen Arbeiten kaum über ein nennenswertes Maß an intrinsischer Motivation verfügen. Aus diesem Grund kann hier intrinsische Motivation auch nicht verdrängt werden. Der Effekt ist neutral. Es lässt sich ableiten, dass die Gefahr einer negativen Beeinflussung durch extrinsische Belohnungen bei Führungskräften, denen tendenziell ein hohes Maß an intrinsischer Motivation zugeschrieben wird, größer ist als bei Arbeitern.

- Ob ein negativer Verdrängungseffekt auftritt, hängt unter anderem davon ab, ob die Mitarbeiter die Einführung leistungsorientierter Entlohnungssysteme als Einschränkung ihrer Selbstbestimmung und als verstärkte Fremdkontrolle interpretieren Diese Gefahr ist besonders bei Mitarbeitern groß, für die finanzielle Anreize von untergeordneter Bedeutung sind (THEUVSEN 2003: 490).

- Eine entgegengesetzte Wirkung ergibt sich, wenn der extrinsische Anreiz als Unterstützung oder Anerkennung der Arbeit angesehen wird. In diesem Fall führen extrinsische Belohnungen also nicht zum „crowding out“, sondern zum „crowding in“ (FREY UND JEGEN 2000: 9). 
- Die Crowding-out-Wirkung kommt vor allem dann zum Tragen, wenn der extrinsische Anreiz von der Person, die ihn erhält, erwartet wird. Gelegentliche Belohnungen wirken folglich anders als regelmäßige.

- Die extrinsische Belohnung muss nicht monetär sein. Auch fiktive Belohnungen wie z.B. Smilies oder Goldsterne für besonders fleißige Schüler können einen negativen Verdrängungseffekt auslösen.

Als Spillover-Effekt wird die Übertragung der Erwartung von Belohnungen auf andere Arbeitsbereiche bezeichnet. Wird man für eine bestimmte Tätigkeit regelmäßig belohnt, so erwartet man diese Belohnung auch für andere Tätigkeiten. Die nicht belohnten Tätigkeiten werden womöglich nach einiger Zeit ohne zusätzliche Belohnung gar nicht mehr ausgeführt (ebenda: 311).

Von weiterer Bedeutung ist die wahrgenommene Fairness. Wird die ausgelobte Belohnung im Vergleich zu einer Referenzgröße als zu niedrig angesehen, kann die intrinsische Motivation ebenfalls sinken. Jedoch kann sie auch positiv oder neutral wirken, nämlich dann, wenn beispielsweise unterdurchschnittliche Lohnerhöhungen mit externen Gründen, wie einer angespannten Wirtschaftslage in Zusammenhang gebracht werden können (ebenda: 311).

Altruismus und die Erfüllung bürgerlicher Pflichten können ebenfalls durch extrinsische Belohnungen verdrängt werden. Dies zeigen Experimente mit Ehrenamtlichen oder NIMBY-Projekte, bei denen den Bürgern Geld als Entschädigung für den Bau des Projektes angeboten wird. Bei NIMBY-Projekten kommt hinzu, dass das Angebot einer Entschädigung auch mit dem Eingeständnis einer damit verbundenen Gefährdung angesehen werden könnte, obwohl dieser Effekt empirisch nicht bestätigt werden kann (FREY UND OBERHOLZER-GEE 1997: 750).

Aus dem Verdrängungseffekt ergeben sich für die Gestaltung von Anreizsystemen in der Landwirtschaft unter Umständen weit reichende Konsequenzen. Da das Auftreten des Verdrängungseffekts von verschiedenen Faktoren abhängt, ist es schwer abzuschätzen, ob extrinsische Anreize bei den Mitarbeitern in der Landwirtschaft zu einem negativen, neutralen oder positiven Effekt auf die Mitarbeitermotivation führen. 
Aus den theoretischen Vorüberlegungen ist davon auszugehen, dass ein negativer Verdrängungseffekt auf den oberen Hierarchieebenen, sofern es diese im landwirtschaftlichen Betrieb überhaupt gibt, eher auftritt. Hier sind die Voraussetzungen für eine verantwortungsvolle, inhaltlich anspruchsvolle Aufgabe, die intrinsische Motivation fördert, in der Regel gegeben.

Aber auch auf der Ebene einfacherer Tätigkeiten in der Produktion sind die Arbeitsabläufe in der Landwirtschaft in der Regel abwechslungsreich und kaum mit monotonen, sich ständig wiederholenden Tätigkeiten verbunden. Deshalb ist auch hier ein gutes Maß an intrinsischer Motivation nicht auszuschließen.

Ob ein extrinsischer Anreiz als Kontrolle oder als Bestätigung angesehen wird, hängt wohl vor allem von der Persönlichkeit des Mitarbeiters ab, so dass hier keine allgemeingültige Antwort gegeben werden kann.

\subsubsection{Reziprozität}

Zwar kann Reziprozität der Akteure als Teileffekt des Verdrängungseffekts angesehen werden, die Implikationen dieser Verhaltensweise für die Mitarbeitermotivation sind jedoch so bedeutend, dass ihr ein eigenes Unterkapitel gewidmet ist.

Reziprozität ist eine Reaktion, die sich auf den Moment bezieht, d.h. Freundlichkeit wird mit Freundlichkeit belohnt, und negatives Verhalten gegenüber einer Person bestraft diese ebenfalls mit negativen Verhaltensweisen. Dabei spielt die Erwartung zukünftiger positiver materieller Belohnungen keine Rolle. Außerdem wird negative oder positive Reziprozität auch ausgeführt, wenn diese mit Kosten verbunden ist (FEHR UND GÄCHTER 2000: 159). Zwar ist das Tit-for-Tat-Spiel von Axelrod und Rapaport (BRANDES ET AL. 1997: 457 f.) ein Beispiel für reziproke Verhaltensweisen, es unterscheidet sich aber von der Reziprozität vor allem dadurch, dass es sich um ein mehrsequentielles Spiel handelt.

Vom Altruismus unterscheidet sich die Reziprozität dadurch, dass dieser nicht zielgerichtet ist. Altruismus stellt keine Bedingungen, während die Reziprozität eine direkte Reaktion auf eine Verhaltensweise darstellt. 
Prominente Beispiele für negative Reziprozität sind Vergeltung in Kriegen oder soziale Isolation von Streikbrechern durch ihre Kollegen. Positive Beispiele von Reziprozität sind die Schwierigkeiten freundlichen Servicekräften Trinkgeld zu verwähren oder von netten Verkäufern nach einer Beratung nichts zu kaufen (FEHR UND GÄCHTER 2000: 161).

Wie sanktionierend Reziprozität wirken kann, zeigt der Bewertungsmechanismus des Internetauktionsportals Ebay: Nach jeder abgeschlossenen Transaktion bewerten sich die Handelspartner gegenseitig. Die Bewertung kann positiv, negativ oder neutral ausfallen. Darüber hinaus gibt es die Möglichkeit, einen schriftlichen Kommentar zum Verhalten des Tauschpartners abzugeben. Für viele Marktteilnehmer hat es sich als positive Strategie herausgestellt, die Handelspartner möglichst positiv zu bewerten, auch wenn man mit der Abwicklung des Geschäfts selbst nicht zufrieden gewesen ist. Ziel ist es nämlich, durch die positive Bewertung des Handelspartners möglichst $100 \%$ positive Bewertungen durch andere $\mathrm{zu}$ erhalten, um als vertrauenswürdiger Handelspartner zu gelten.

Die Konsequenzen negativer Reziprozität zeigen Experimente mit dem UltimatumSpiel. Ein robustes Ergebnis der Spieldurchführung in verschiedenen Ländern ist es, dass Anbieter, die weniger als $30 \%$ der insgesamt zur Verfügung stehenden Summe ihrem Partner anbieten, mit einer sehr hohen Wahrscheinlichkeit abgewiesen werden. Das Motiv hinter dieser Ablehnung ist es, dass eine ungünstigere Aufteilung als unfair angesehen wird (FEHR UND GÄCHTER 2000: 161).

Nach den Angaben von FEHR UND GÄCHTER besteht Konsens darüber, dass die Bereitschaft schlechte Verhaltensweisen zu bestrafen größer ist als die Bereitschaft positive Handlungen zu belohnen.

In der Literatur werden verschiedene Erklärungsmöglichkeiten für das relativ robuste Auftreten von Reziprozität gegeben (ebenda: 162 f):

- Der Wunsch Gleichheit im Austauschverhältnis zu erreichen. In diesem Fall wäre Reziprozität durch Adams Equity Theorie zu erklären.

- Das Bedürfnis feindliche Absichten zu bestrafen und freundliche zu belohnen. 
- Die Reaktion auf einen bestimmten Personentyp und nicht auf die von dieser Person gehegten Absichten.

- Eine Form von „bounded rationality“.

Für verschiedene Situationen hat reziprokes Verhalten Bedeutung. Die Entscheidung, ob Menschen zu öffentlichen Gütern einen Beitrag leisten, hängt auch von Reziprozität ab. Ein freiwilliger Beitrag zu einem öffentlichen Gut wird hier als eine Form positiver Reziprozität definiert, wenn dieser sich dadurch ergibt, dass andere Personen ebenfalls aus freien Stücken hierzu etwas beitragen. Trittbrettfahrerverhalten ist jedoch nicht per se Ausdruck von negativer Reziprozität, da diese im Fall öffentlicher Güter nicht bestraft wird. Die Bedeutung negativer Reziprozität steigt jedoch, wenn man Trittbrettfahren bestrafen kann.

Reziprozität hat für die Gestaltung von Entlohnungssystemen und für die leistungsorientierte Entgeltgestaltung im speziellen folgende Bedeutung. Sie kann zu Lohnrigidität führen, d.h. ein einmal eingeführtes Lohnniveau kann auch in Zeiten ungünstiger wirtschaftlicher Rahmenbedingungen kaum mehr abgesenkt werden, da die Mitarbeiter dies als feindselige Handlung interpretieren können (FEHR UND GÄCHTER 2000: 175).

Die Einführung leistungsorientierter Entgeltsysteme ist eine Maßnahme, um implizite und unvollständige Verträge explizit zu machen. Ziel ist es also, ein bestimmtes Aktivitätsniveau der Mitarbeiter durch Belohnungen oder Sanktionen zu erreichen. Diese Wirkung wird jedoch nicht immer erzielt oder sie ist mit hohen Überwachungskosten verbunden, wie verschiedene Experimente ergeben haben (ebenda: 168 ff.). Reziprozität kann außerdem dann eine negative Wirkung leistungsorientierter Entlohnung hervorbringen, wenn kooperatives Verhalten eine unabdingbare Eigenschaft der zu erfüllenden Tätigkeit ist.

Schafft man eine experimentelle Prinzipal-Agenten-Situation, in der sowohl Prinzipal als auch Agent die Wahl zwischen impliziten und expliziten Verträgen haben, so wählen im Experiment sowohl Prinzipal als auch Agent überwiegend implizite Verträge (ebenda: 176 f.). 
Es lässt sich schlussfolgern, dass die Intentionen und Verhaltensweisen des Gegenübers für die Entscheidung von Menschen höchst relevant sind. Hierzu gehört zum Beispiel die Würdigung der Absichten, die hinter einem bestimmten Verhalten stehen. Die Belohnung positiver Arbeitseinstellung, die auf intrinsischen Beweggründen beruht, durch extrinsische Anreize kann als Bruch eines impliziten Vertrages interpretiert werden (FREY UND OSTERLOH 1997: 311).

\subsubsection{Experimente zur Bedeutung von Fairness}

In Kapitel 3.2.3 wurde die motivationstheoretische Bedeutung von Fairness dargelegt. Auf die empirische Prüfung der Bedeutung von Fairness auf das Verhalten von Menschen mit Hilfe der experimentellen Ökonomik soll im Folgenden eingegangen werden.

Eine mögliche Erklärung für das Auftreten von Reziprozität ist das Gerechtigkeitsempfinden. Experimentelle Ökonomen konnten in verschiedenen Experimenten feststellen, dass Fairness ein nicht $\mathrm{zu}$ vernachlässigender Aspekt menschlichen Verhaltens und ökonomisch relevant ist. Die von FEHR und Co-Autoren durchgeführten Experimente zu Fairnessnormen sind im Wesentlichen dieselben wie die Experimente zur Reziprozität. Den Einfluss von Fairness auf den Ausgang von Spielen zeigen Experimente mit dem Ultimatum-, Diktator-, Trust- und Gift-Exchange-Spiel (FEHR UND SCHMIDT 2001). Fairness spielt einen entscheidenden Einfluss auf den Ausgang der Spiele. Ein wesentliches Ergebnis ist es, dass sich Menschen im Ausmaß ihres Gerechtigkeitsempfindens unterscheiden, es kommt jedoch in der Mehrzahl der Spielsituationen dazu, dass Menschen mit ihrem Gegenüber teilen.

Dass Fairness einen wichtigen Einfluss auf das Entscheidungsverhalten von Menschen haben kann, zeigen auch Experimente, die die Forschergruppe um KAHNEMAN zur Prüfung der Verlustaversion durchgeführt hat. Zum Beispiel werden Preiszuschläge, die z.B. auf schlechten wirtschaftlichen Rahmenbedingungen beruhen, als ungerechter angesehen als die Abschaffung eines Preisnachlasses im gleichen Umfang (KAHNEMAN ET AL. 1991: 204). 
Zwar spielt Fairness in verschiedenen Entscheidungssituationen eine wichtige Rolle als soziale Norm, die Bedeutung für den Arbeitsmarkt und die Wirkung von Anreizsystemen wurde jedoch isoliert von verschiedenen Autoren untersucht. Die Ergebnisse von FEHR UND GÄCHTER (2000) wurden schon im vorausgehenden Kapitel 3.3.3 dargestellt.

Sкотт (2005) hat darüber hinaus die Bedeutung von Fairness für den Arbeitsmarkt herausgearbeitet und stellt fest, dass Fairness eine Ursache für Hysterese ${ }^{14} \mathrm{im}$ Arbeitsmarkt sein kann. Es sei seiner Meinung nach unstrittig, dass der Lohn einer Vergleichsgruppe als Referenzpunkt für die Beurteilung des eigenen Lohnniveaus diene. Einer besonderen Bedeutung komme außerdem dem Status Quo der eigenen Bezahlung zu. Arbeiter orientierten sich demnach an multiplen Referenzpunkten (ebenda: 306 f.).

Trägheiten im Arbeitsmarkt können folglich dadurch zustande kommen, dass ein einmal eingeführtes Lohnniveau auch bei ungünstigen Rahmenbedingungen nicht abgesenkt werden kann, da diese Handlung als ungerecht angesehen wird und sich somit negativ auf die Mitarbeitermotivation auswirken kann. Praktische Konsequenz ist Lohnrigidität, die zum einen eine künstlich erhöhte Arbeitslosigkeit nach sich zieht, da nicht mehr nach der Grenzproduktivitätstheorie eingestellt wird.

Als Konsequenz aus der Arbeitslosigkeit ergibt sich eine induzierte Überqualifikation und ein Nicht-Zusammenpassen von Arbeitsanforderung und Qualifikation.

Die Ausführungen des vorliegenden Kapitels sind zum Teil in ihren Aussagen zum Menschenbild recht unterschiedlich oder teils auch widersprüchlich, dass Fairnessaspekte jedoch ein sehr robustes menschliches Phänomen sind, zeigt, dass sie auch in neoklassische Theorien wie die Effizienzlohntheorie inzwischen explizit Eingang gefunden haben (vgl. Kapitel 3.1.3).

BOLTON UND OCKENFELS (2000) haben darüber hinaus ein ökonomisches Modell aufgestellt, dass es vermag, eine Verbindung zwischen auf Gleichheit bedachten, kompetitiven und reziproken Verhaltensweisen herzustellen und somit die Kontroversität zwischen dem neoklassischen und dem sozialpsychologischen

\footnotetext{
${ }^{14}$ Unter dem Begriff Hysterese versteht man in den Wirtschaftswissenschaften beobachtbare Trägheiten im Arbeitsmarkt.
} 
Menschenbild zumindest teilweise aufzulösen. Das so genannte ERC-Modell ${ }^{15}$ basiert auf den vorwiegend in Ultimatum- und Diktatorspielen gewonnen Datensätzen experimenteller Ökonomen, wie z.B. FEHR und Co-Autoren. Danach hängt es von den Rahmenbedingungen ab, ob sich die Spieler fair, reziprok oder kompetitiv verhalten.

\subsection{Erfolgsbedingungen leistungsorientierter Lohngestaltung}

Die in Kapitel 3 vorgestellten verschiedenen theoretischen Ansätze zur Gestaltung von Anreizsystemen sind zum Teil recht widersprüchlich in ihrer Aussage. Es soll aber im Folgenden der Versuch angestellt werden, einige Erfolgsbedingungen für eine leistungsorientierte Lohngestaltung abzuleiten.

In Abbildung 10 sind die Erfolgsbedingungen leistungsorientierter Entlohnung und deren Wirkung auf die Effektivität leistungsorientierter Entgelte dargestellt, wie sie THEUVSEN 2003 modellhaft formuliert hat. Ein Pluszeichen bedeutet, dass ein Einflussfaktor die abhängige Variable positiv beeinflusst, ein Minuszeichen symbolisiert den umgekehrten Zusammenhang.

\section{ABBILDUNG 10: ERFOLGSBEDINGUNGEN LEISTUNGSORIENTIERTER ENTLOHNUNG}

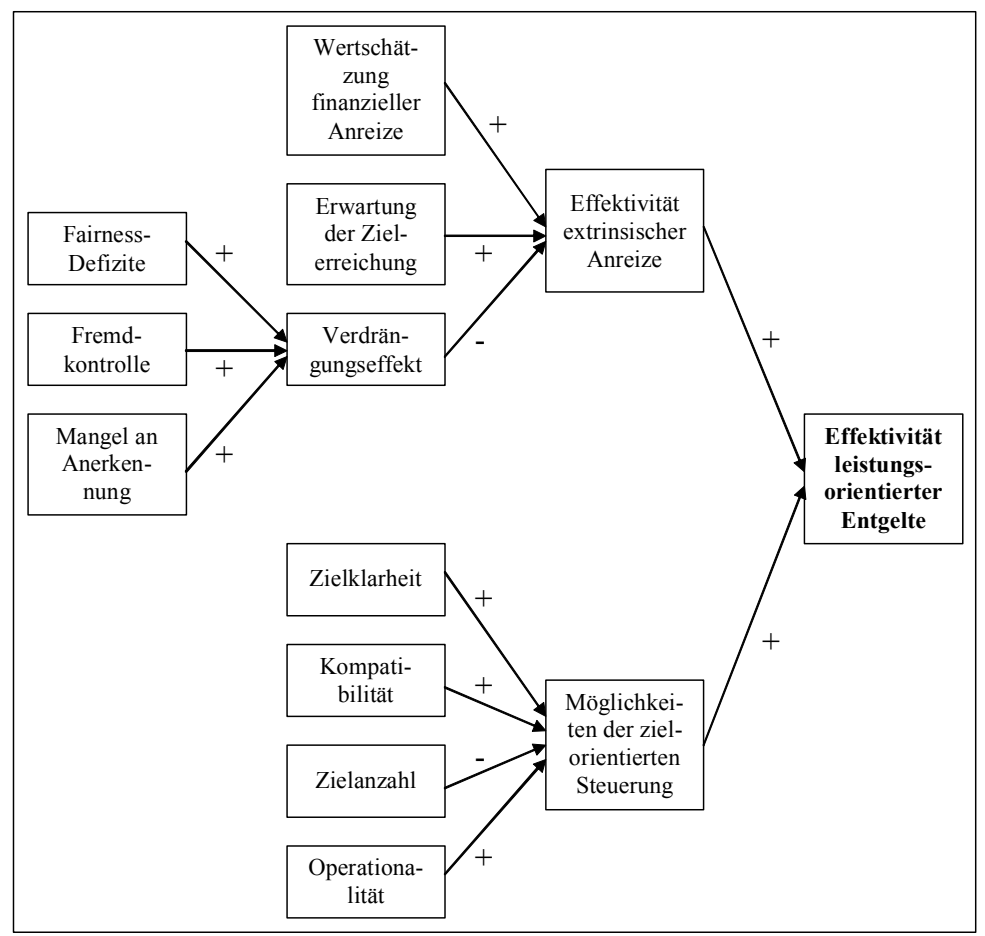

Quelle: THEUVSEN 2003: 499

${ }^{15}$ Abgekürzt nach „Equity“, „Reciprocity“ und „Competition“. 
Durch leistungsorientierte Entlohnungssysteme werden Mitarbeitern finanzielle Anreize geboten, um sie zur Ausführung bestimmter Handlungen zu motivieren. Die Motivationswirkung beruht hierbei auf der Stärkung extrinsischer, auf Gewährung von Belohnungen beruhender Motivation.

Die Effektivität extrinsischer Anreize ist dann besonders hoch, wenn die folgenden Bedingungen zutreffen:

- Die Mitarbeiter müssen die in Aussicht gestellten finanziellen Anreize schätzen und diese auch mit einer hohen subjektiven Wahrscheinlichkeit erreichen können. Diese Erfolgsbedingung ist eine Konsequenz aus dem Motivationsmodell von PORTER UND LAWLER.

- Das durch leistungsabhängige Entlohnung bewirkte Mehr an extrinsischer Motivation darf nicht durch ein Weniger an intrinsischer, aus der gestellten Arbeitsaufgabe selbst resultierende Motivation überkompensiert werden (Verdrängungseffekt). Hier ist außerdem abzuschätzen, ob die betreffenden Mitarbeiter die leistungsorientierte Entlohnung als unfair ansehen. Fairness wird hierbei in Bezug auf die Entlohnung einer Referenzgruppe und den eigenen Status-Quo angesehen. Diese Bedingung leitet sich zum einen aus Adams Equity Theorie, aber auch aus der experimentellen Ökonomik ab. Außerdem stellt sich die Frage, ob das Entlohnungssystem als positive Unterstützung oder als zunehmende Fremdkontrolle und Aberkennung der bisherigen Leistung interpretiert wird.

Neben der Wirksamkeit extrinsischer Anreize sind auch die Möglichkeiten der zielorientierten Steuerung für die Effektivität leistungsorientierter Entgelte von Bedeutung. Diese Möglichkeiten werden im Wesentlichen von vier Bedingungen beeinflusst:

\section{Zielklarheit}

Die Unternehmensleitung muss den Mitarbeitern die angestrebten Ziele klar benennen können und die Ziele auch deutlich kommunizieren. 


\section{Zielrelevanz und-kompatibilität}

Die von der Unternehmensleitung festgelegten Ziele müssen für die Oberziele des Unternehmens von Relevanz sein. Die definierten Ziele in den unteren Hierarchieebenen müssen mit den oberen Zielen der Unternehmensleitung kompatibel sein. Ist diese Bedingung verletzt, so kann dies in zweierlei Hinsicht negative Konsequenzen für den Erfolg eines leistungsorientierten Lohnsystems haben. In dem Modell von PORTER UND LAWLER wurde deutlich, dass eine Anreizwirkung nur zu erwarten ist, wenn der Mitarbeiter den Eindruck gewinnt, dass seine Anstrengung im Zusammenhang mit der erwarteten Leistung steht. Zum zweiten ist es wichtig, dass das Unterziel nicht mit dem Oberziel kollidiert. Praxisbeiträge in landwirtschaftlichen Fachzeitschriften zeigen, dass es durchaus zu solchen Konflikten kommen kann. Als ein Erfolgsindikator wird zum Beispiel die Anzahl der aufgezogenen Ferkel pro Sau und Jahr herangezogen (GRIESEL 2004: 7). Die Mitarbeiter erzielen eine Erhöhung des Leistungsparameters durch eine Erhöhung der Remontierungsrate. Ältere Sauen werden schneller durch Jungsauen ersetzt, denn Jungsauen erreichen höhere Aufzuchtraten. Eine Erhöhung der Remontierungsrate führt allerdings zu höheren Aufzuchtkosten, so dass es die Erreichung des Oberziels in Frage gestellt.

\section{Zielanzahl}

Im oben genannten Beispiel rät der Autor zur Einbeziehung weiterer Leistungsindikatoren zur Bemessung des Leistungsentgelts, um den negativen Auswirkungen gegenzusteuern (ebenda: 7). Es ist jedoch zu beachten, dass zu viele Ziele unpraktikabel sind, da mit wachsender Zielanzahl die Mitarbeiter zunehmende Schwierigkeiten bekommen, die Zielwirkungen verschiedener Handlungen abzuschätzen.

\section{Operationalität}

Die neoklassischen Forschungsansätze gehen von einem rational handelnden Menschen aus, der sich vor allem von seinem Eigeninteresse leiten lässt. Die Prinzipal-AgentenTheorie und Transaktionskostentheorie besagen, dass die Leistung beziehungsweise der Arbeitseinsatz von beiden Parteien beobachtbar und objektiv messbar sein sollte. Außerdem führen komplexe Kennzahlensysteme bei der Bemessung von Leistungsentgelten $\mathrm{zu}$ hohen Transaktionskosten, die durch den Kontrollaufwand bedingt sind. Diese können leicht einen möglichen positiven Motivationseffekt aufwiegen. 


\section{Gestaltungsmöglichkeiten materieller Anreizsysteme}

Im folgenden Kapitel werden verschiedene Gestaltungsmöglichkeiten materieller Anreizsysteme dargestellt. Die materiellen Anreizsysteme werden in diesem Kapitel nach der Art ihrer Bemessungsgrundlage klassifiziert (vgl. Abbildung 11). Es werden zunächst die Vergütungssysteme mit inputabhängiger Bemessungsgrundlage erklärt und im Anschluss daran, die Entgeltsysteme mit outputabhängiger Bemessungsgrundlage. Die Mischformen zwischen input- und outputabhängiger Bemessungsgrundlage werden bei den outputabhängigen Entlohnungssystemen diskutiert.

ABBILDUNG 11: MATERIELLE ANREIZSYSTEME IM ÜBERBLICK

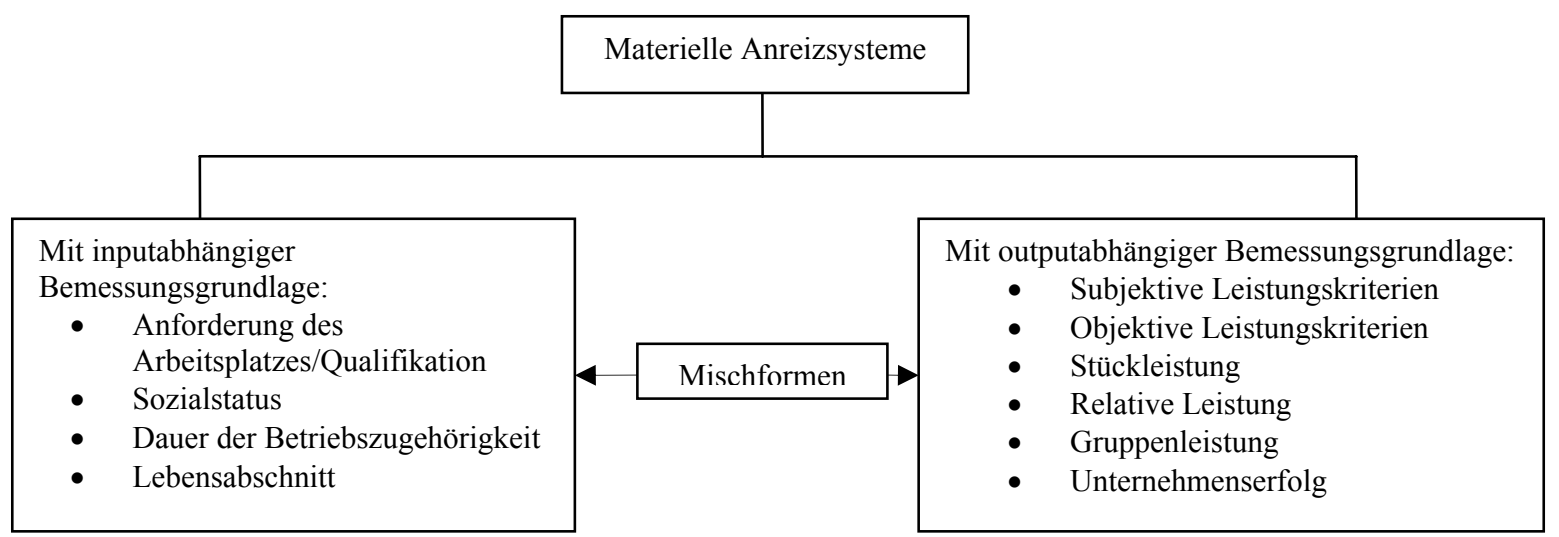

Quelle: Eigene Darstellung 


\subsection{Inputorientierte Entlohnungssysteme}

\subsubsection{Anforderungsabhängige Löhne}

Zeitentgelte beruhen in der Regel auf den für die Tätigkeit bzw. den Arbeitsplatz notwendigen Anforderungen und der absolvierten Arbeitszeit. Stundenlohn und Gehalt sind anforderungsabhängige Löhne und unterscheiden sich durch ihre Abrechnungsbasis, z.B. Stunde versus Monat. Anforderungsabhängige Löhne bieten keinen expliziten Leistungsanreiz.

$\mathrm{Da}$ für die Bestimmung des Zeitlohns die Anforderungen eines bestimmten Arbeitsplatzes definiert werden müssen, kommen verschiedene Arbeitsbewertungsverfahren zum Einsatz. Sie können kategorisiert werden als summarische Verfahren oder analytische Verfahren. Können die Anforderungen auf kontinuierlichen Skalen gemessen werden, so handelt es sich um eine Reihung, ansonsten um eine Stufung (SCHOLZ 2000: 736).

$\mathrm{Zu}$ den summarischen Verfahren gehört das Rangfolgeverfahren, dem eine kardinale Messung zugrunde liegt, und das Lohngruppenverfahren, bei dem die Einstufung ordinal erfolgt (EISENFÜHR UND THEUVSEN 2004: 221). Von den summarischen Verfahren ist für die Landwirtschaft das Lohngruppenverfahren von besonderer Bedeutung. Es ist in zahlreichen Tarifverträgen verankert. Die Tabelle 9 zeigt die Lohngruppenbeschreibung und die Stundenlöhne nach dem Lohntarifvertrag der Landarbeiter in Schleswig-Holstein. Es werden Lohngruppen für Erntehelfer von den Lohngruppen der ständig beschäftigten Arbeitnehmer unterschieden. Bei den Erntehelfern gibt es nur zwei Lohngruppen, eine für leichte und eine für schwere Arbeiten. Die Einteilung in die Lohngruppen erfolgt nach Anforderung und Ausbildung. 
TABELLE 9: LOHNGRUPPENBESCHREIBUNG UND STUNDENLÖHNE FÜR STÄNDIG BESCHÄFTIGTE LANDARBEITER IN SCHLESWIG-HOLSTEIN

\begin{tabular}{|c|l|c|}
\hline Lohngruppe & Beschreibung der Anforderungen & Stundenlohn in Euro \\
\hline \multirow{3}{*}{1} & Hilfsarbeiter für leichte Arbeiten & 6,03 \\
& über 18 Jahre & 5,46 \\
& bis 18 Jahre & 5,10 \\
\hline \multirow{2}{*}{2} & bis 16 Jahre & \\
& Angelernte Arbeiter für leichte Arbeiten & 6,47 \\
& über 18 Jahre & 5,83 \\
& bis 18 Jahre & 5,55 \\
\hline \multirow{3}{*}{3} & bis 16 Jahre & 7,87 \\
& Hilfsarbeiter für schwere Arbeiten & 6,27 \\
& über 18 Jahre & 5,55 \\
\hline \multirow{2}{*}{4} & bis 18 Jahre & \\
& bis 16 Jahre & 8,69 \\
\hline 5 & Landarbeiter & 7,87 \\
\hline 6 & über 18 Jahre & 9,26 \\
\hline \multirow{2}{*}{7} & bis 18 Jahre & 9,91 \\
\hline & Freckerfahrer und Maschinenführer & \\
\hline & Handwerker & 10,63 \\
\hline
\end{tabular}

Quelle: Eigene Darstellung nach dem Lohntarifvertrag für Landarbeiter in Schleswig-Holstein vom 22.4 .200

Nach Angaben der SÄCHSISCHEN LANDESANSTALT FÜR LANDWIRTSCHAFT (2002: 35 f.) werden bei betrieblich fixierten Lohngruppen auch Kriterien des Arbeitsverhaltens wie Einsatz- und Leistungsbereitschaft, Verantwortungsbewusstsein oder die Arbeitsqualität zur Ein- bzw. Umgruppierung herangezogen. In diesem Fall handelt es sich beim Lohngruppenverfahren jedoch nicht mehr um ein input- sondern um ein outputorientiertes Entlohnungssystem.

Bei den analytischen Verfahren unterscheidet man das Rangreihenverfahren (kardinale Messgrundlage) und das Stufenwertzahlverfahren (ordinale Messgrundlage). Das Rangreihenverfahren legt der Arbeitsbewertung verschiedene Anforderungsarten zugrunde, die durch das „Genfer Schema“ vereinheitlicht wurden. Hierzu zählen geistiges und körperliches Fachkönnen, geistige und körperliche Belastung, Verantwortung und Arbeitsbedingungen (EISENFÜHR UND THEUVSEN 2004: 222). Bei der Arbeitsbewertung mit dem Rangreihenverfahren wird für jeden Arbeitsplatz bewertetet, wie hoch (auf einer Skala von 0 bis 100) die jeweiligen Anforderungen sind. Die Bedeutung verschiedener Anforderungen kann auch noch gewichtet werden. Der Arbeitsplatz mit den wenigsten Punkten erhält den Mindestlohn, der mit den meisten Punkten den Maximallohn. Die anderen Arbeitsplätze werden nach der Anzahl ihrer 
Punkte eingestuft. Das Stufenwertzahlverfahren funktioniert ähnlich, es werden aber statt Punkten Anforderungsstufen vergeben. Analytische Arbeitsbewertungsverfahren haben den Vorteil, dass sie für Arbeitnehmer besser nachvollziehbar sind. Der Nachteil ist, dass sie sehr aufwändig sind und bei flexiblen Arbeitsprozessen problematisch sind, wenn die Mitarbeiter in einem Unternehmen verschiedene Funktionen erfüllen. Da die Flexibilität des Arbeitsprozesses ein wesentliches Charakteristikum der Landwirtschaft ist (siehe Kapitel 2.1), spielen analytische Arbeitsbewertungsverfahren eine untergeordnete Rolle.

Der Zeitlohn hat unter den folgenden Rahmenbedingungen besondere Bedeutung und Vorteile (vgl. EISENFÜHR UND THEUVSEN 2004: 214, KUHLMANN 2003: 279):

1) Das Arbeitsergebnis ist vom Mitarbeiter nicht wesentlich beeinflussbar.

2) Die Leistung ist nicht zuverlässig messbar.

3) Es gibt vielfältige Leistungsdimensionen.

4) Die Tätigkeit erfordert besondere Sorgfalt.

5) Das Arbeitstempo ist konstant und/oder wird z.B. durch eine Maschine diktiert.

Der große Vorteil des Zeitentgelts ist sein geringer Kontroll- und Verwaltungsaufwand. Bei vielen Tätigkeiten ist es schwierig, das Ergebnis der Arbeit zu messen. Die Messung der Arbeitsleistung kann daher sehr aufwändig und auch unvollständig sein, da nicht alle leistungsrelevanten Parameter erfasst werden können (WOLFF UND LAZEAR 2001: 274). Dies betrifft vor allem Arbeiten auf Führungsebene, denn einige Managementfähigkeiten wie Kreativität, Entscheidungsfreude, Organisationsfähigkeit lassen sich kaum an brauchbaren Indikatoren messen.

Die Wahl der Abrechnungsbasis (Stunde, Monat oder Jahr) hängt von der Tätigkeit ab, die es zu entlohnen gilt. Arbeiter werden meist auf Stundenbasis entlohnt, während Angestellte und vor allem Führungskräfte ihren Zeitlohn pro Monat oder pro Jahr erhalten. Je schwieriger es ist, die Tätigkeit zu beschreiben, desto länger wird die Abrechnungsperiode (WOLFF UND LAZEAR 2001: 280). Führungskräfte haben außerdem im Vergleich zu Arbeitern mehr Einfluss auf ihre Zeiteinteilung. Ihre Tätigkeit 
überschreitet vielfach die normalen Arbeitszeiten, so dass eine Entlohnung pro Stunde für das Unternehmen auch sehr kostspielig werden kann.

\subsubsection{Sozialstatus- und lebensabschnittsabhängige Löhne}

Neben den Anforderungen des Arbeitsplatzes finden in Unternehmen auch Entgeltdifferenzierungen nach dem Sozialstatus oder dem Lebensabschnitt statt.

Zum Sozialstatus gehören z.B. der Familienstand oder die Dauer der Betriebszugehörigkeit. Einige Tarifverträge in der Landwirtschaft differenzieren das Gehalt bzw. den Lohn nach dem Familienstand. So sieht z.B. der Lohntarifvertrag für Melker in Schleswig-Holstein eine unterschiedlich hohe Grundentlohnung in Abhängigkeit vom Familienstand vor. Verheiratete männliche Melker erhalten einen höheren Grundlohn als Unverheiratete, eine Dienstwohnung und ein Milchdeputat.

Die Berücksichtigung des Familienstandes - hier exemplarisch am Beispiel des Tarifvertrags für Melker verdeutlicht - bei der Entgelthöhe ist motivationstheoretisch dann begründbar und möglicherweise ökonomisch sinnvoll, wenn der Mitarbeiter eine Familie alleine versorgen muss. Aus der Perspektive von Maslows Bedürfnishierarchie muss der Lohn hier die Existenzbedürfnisse von mehreren Familienmitgliedern abdecken, bevor die Befriedigung weiterer Bedürfnisse eine Motivationswirkung entwickeln kann.

Die Dauer der Betriebszugehörigkeit bei der Entlohnung wird zum Beispiel im Gehaltstarifvertrag der Salzgitter Güterverwaltung $\mathrm{GmbH}$ berücksichtigt. Hier wird innerhalb der Lohngruppen differenziert zwischen Angestellten, die bis 2 Jahre, 2 bis 4 Jahre und länger als 4 Jahre im Betrieb beschäftigt sind. Das Einbeziehen der Betriebsdauer in die Lohnfindung ist ökonomisch dadurch $\mathrm{zu}$ begründen, dass der Verlust eines langjährigen Mitarbeiters für das Unternehmen teuer ist, da gerade in der Anfangszeit der Beschäftigung eines Mitarbeiters viele Investitionen getätigt werden müssen. Diese beinhalten z.B. die Anwerbung, Einarbeitung und Aneignung unternehmensspezifischen Know-hows.

Lebensabschnittsabhängige Löhne werden auch als Senioritätslöhne bezeichnet. Sie stellen eine verzögerte ${ }^{16}$ Form der Entlohnung dar. Dies bedeutet, dass die

${ }^{16}$ Im englischen Sprachraum wird diese Entlohnungsform mit „,deferred payments“ oder „,deferred compensation" bezeichnet. 
Arbeitnehmer zu Anfang der Beschäftigung im Unternehmen einen Lohn erhalten, der geringer ist als das Wertgrenzprodukt ihres Arbeitseinsatzes. Das Lohnprofil ist steil, d.h. nach einigen Jahren erhalten die Mitarbeiter einen im Vergleich zum Anfangsgehalt erhöhten Lohn. Verzögerte Zahlungen sind vergleichbar mit einem Firmenkredit, den der Arbeitnehmer dem Unternehmen gewährt. Um diesen Kredit zurückzuerhalten, muss der Arbeitnehmer möglichst lange im Unternehmen verbleiben. Dadurch soll ein Leistungsanreiz gegeben werden, der "Bummeln" über einen langen Beschäftigungszeitraum verhindert (MORETTI UND PERLOFF 2002: 1144, MILGROM UND ROBERTS 1992: 433).

Senioritätslöhne werden häufig so gestaltet, dass der Lohnanstieg in der früheren Phase des Erwerbslebens größer ist als in der späteren Phase. Eine Anreizwirkung entwickelt sich folglich eher bei jüngeren als bei älteren Arbeitnehmern, da es eine Obergrenze gibt, ab der keine weiteren lebensabschnittsabhängigen Lohnsteigerungen zu erwarten sind. Der Vorteil von Entlohnung nach Seniorität ist das unstrittige Messkriterium, denn die Dauer der Betriebszugehörigkeit ist einfach zu messen (BILliKOPF 2003: 42). Das Risiko für den Arbeitnehmer besteht bei Senioritätslöhnen darin, dass das Unternehmen bankrott gehen könnte und damit die erwartete Lohnsteigerung im Alter ausbleibt (BACKES-GELLNER ET AL. 2001: 269 f.).

Milgrom Und Roberts (1992: 432 f.) sehen den Vorteil von Senioritätslöhnen vor allem in der Entlohnung von Führungskräften, da die Leistung der Führungskräfte in Bezug auf den Unternehmenserfolg nur langfristig messbar ist. Sie berichten von einem Senioritätssystem der Firma BellSouth, einer amerikanischen Telefongesellschaft. Zu Beginn der Beschäftigung im Unternehmen haben die Mitarbeiter die Möglichkeit, $25 \%$ ihres Lohnes in ein Sparkonto zu legen. Das Unternehmen stockt diesen Betrag auf. Wenn ein Mitarbeiter aus Altersgründen oder aufgrund einer Frührentevereinbarung ausscheidet, erhält er aus diesem Sparkonto den ganzen Betrag mit Verzinsung. Wechselt er das Unternehmen, so erhält er nur seinen Sparbetrag mit der marktüblichen Verzinsung zurück (ebenda: 347).

Nach Angaben der F.A.Z. (2007: C1) ist die Berücksichtigung des Senioritätsprinzips in Unternehmen weit verbreitet. 52 \% der Unternehmen verwenden diese Vergütungsform.

Die befragten Unternehmen wollen aber in Zukunft ihr Entgeltsystem ändern und die Bezahlung vom Lebensalter entkoppeln (ebenda). Eine Anpassung der 
Entlohnungskurve an den tatsächlichen Leistungsverlauf empfiehlt SCHOLZ (2000: 761 f.). Gängige Vergütungsmodelle sind nach seiner Erfahrung geprägt von einer relativen Überbezahlung am Anfang und am Ende des Erwerbslebens. In der Anfangsphase werden junge Mitarbeiter, die über wenig Erfahrung verfügen, eher für ihr Potenzial als für ihre tatsächliche Leistung bezahlt. In der Schlussphase des Erwerbslebens für die in der Vergangenheit realisierten Verdienste. Folglich ist in der Phase der höchsten Leistung des Mitarbeiters, also in der mittleren Lebensphase, das Verhältnis von Bezahlung zu Leistung am unadäquatesten. SCHOLZ nimmt aber an, dass auch in Zukunft die Vergütungssysteme im Hinblick auf die lebensabschnittsabhängigen Bedürfnisse der Mitarbeiter nicht flexibler werden (F.A.Z.: C1).

\subsection{Outputorientierte Entlohnungssysteme}

\subsubsection{Leistungszulage}

Die Leistungszulage stellt eine Mischform aus in- und outputorientierten Entlohnungssystemen dar. Sie besteht aus einem anforderungsabhängigen Grundlohn und einer variablen Leistungszulage, die auf personenbezogenen Leistungsbeurteilungen beruht. Leistungszulagen auf der Grundlage von Arbeitsbewertungen sind in Lohntarifverträgen zu finden (REISCH 1992).

Verschiedene Beurteilungsmerkmale können für die Bemessung der Leistungszulage herangezogen werden. Hierzu gehören die Arbeitsquantität (Intensität, Wirksamkeit und Arbeitsweise), Arbeitsqualität (z.B. Sorgfalt im Umgang mit Maschinen), das Einhalten von rechtlichen Schutzvorschriften, Führungs-, Verhaltens- und Persönlichkeitsmerkmale (REISCH 1992: 2363, SCHOLZ 2000: 745).

Die aufgezeigten Beispiele für Leistungszulagen zeigen den subjektiven Charakter dieser Entlohnungsform. Deshalb sollte sie nur dort Anwendung finden, wo es nicht möglich ist, objektive Bewertungskriterien zu finden.

Außerdem ist auf eine periodisch stattfindende Leistungsbeurteilung mit Mitarbeitergespräch zu achten, um dem Mitarbeiter Gelegenheit zu geben, die Bemessungsgrundlage der Leistungszulage zu verstehen.

Die SÄChSISCHE LANDESANSTALT FÜR LANDWIRTSCHAFT (2002: 46) zeigt ein Praxisbeispiel für die Gewährung von Leistungszulagen in der Pflanzenproduktion auf. 
Im genannten Beispiel erhalten die Mitarbeiter der Pflanzenproduktion während der Arbeitsspitze einen Zuschlag von maximal 30 \% zum Grundlohn für hohe Leistungen mit guter Arbeitsqualität. Dieses Praxisbeispiel verdeutlicht die Problematik der Subjektivität von Leistungszulagen. Hohe Leistungen und eine gute Arbeitsqualität sind keine objektiv messbaren Leistungsindikatoren, so dass die Wahrscheinlichkeit groß ist, dass Mitarbeiter, die den Zuschlag nicht erhalten, die Vergütungsregelung als unfair ansehen. Dies würde nach dem Modell von PORTER UND LAWLER (vlg. Kapitel 3.2) dazu führen, dass der durch das Vergütungssystem beabsichtigte Motivationsanreiz mittel- bis langfristig verfehlt wird.

\subsubsection{Prämienlohn}

Der Prämienlohn ist eine Kombination aus Grundlohn und einer Prämie, die auf „objektiven“ Mehrleistungen beruht. Ein Vorteil des Prämienlohns im Vergleich zum Akkordlohn ist die geringere Volatilität des Arbeitseinkommens und die weniger ausgeprägte Störanfälligkeit gegenüber stochastischen Einflüssen, da ein Grundlohn unabhängig vom Output immer gewährt wird.

TABELLE 10: PRÄMIENARTEN UND -INDIKATOREN

\begin{tabular}{|l|l|}
\hline \multicolumn{1}{|c|}{ Arten von Prämien } & \multicolumn{1}{|c|}{ Indikatoren } \\
\hline Mengenleistungsprämien & Steigerung der Mengenleistung \\
\hline Qualitätsprämien & $\begin{array}{l}\text { Steigerung der Produktqualität (z.B. Milchqualitätsprämie, } \\
\text { Milchinhaltsstoffe; Zellzahlen) }\end{array}$ \\
\hline Ersparnisprämie & $\begin{array}{l}\text { Sparsamer Einsatz von Roh- Hilfs- und Betriebsstoffen (Diesel, Öl, } \\
\text { Werkzeug, Schmierstoffe) }\end{array}$ \\
\hline Nutzungsgradprämie & Steigerung der Kapazitätsauslastung \\
\hline Sonderformen & Termineinhaltung, Unfallverhütung \\
\hline
\end{tabular}

Quelle: Eigene Darstellung in Anlehnung an ScHOLZ 2000: 747, KUHLMANN 2003: 281

Tabelle 10 verdeutlicht, dass es eine Vielzahl von Indikatoren gibt, auf denen der Prämienlohn basieren kann. Um negative Wechselwirkungen zwischen verschiedenen Indikatoren zu vermeiden, werden zum Teil komplexe Vergütungssysteme entworfen, die aus dem Blickwinkel der Nachvollziehbarkeit motivationstheoretisch zweifelhaft sind, da die Anreizwirkung darunter leiden kann. 


\subsubsection{Akkordlohn}

Der Akkordlohn ist im Gegensatz zur Leistungszulage oder dem Prämienlohn eine rein leistungsabhängige Entlohnungsform.

Man unterscheidet den Geldakkord vom Zeitakkord. Beim Geldakkord erhält der Arbeiter pro geleistetes Stück einen zuvor definierten Geldbetrag.

In die Berechnung des Zeitakkords fließen zwei Größen ein. Zum einen der Akkordgrundlohn, der sich aus dem tariflich festgelegten Zeitlohn bemisst zuzüglich eines Akkordzuschlags, der etwa 30 bis $50 \%$ beträgt. Akkordgrundlohn und -zuschlag ergeben zusammen den Akkordrichtsatz in Euro/h. Dividiert durch die Anzahl der Stücke, die pro Stunde produziert werden können, ergibt sich das Entgelt/Stück. Der Geldfaktor besagt, wie viel Euro/Minute bezahlt werden (EISENFÜHR UND THEUVSEN 2004: 215). Besondere Bedeutung bei der Bemessung des Akkords kommt Arbeitsstudien $\mathrm{zu}$, auf deren Grundlage festgelegt wird, wie lange die Erstellung einer Arbeitseinheit (eines Werkstücks) dauert.

Der Akkordlohn kann auf individueller oder Gruppenbasis bezahlt werden. Der letztere Fall findet Anwendung, wenn der Einzelne das Ergebnis nicht alleine beeinflusst. Die Vor- und Nachteile von Gruppenentlohnungen werden in Kapitel 4.2.4 diskutiert.

Ein sinnvoller Einsatz des Akkordlohns ist an verschiedene Bedingungen geknüpft (ebenda: 216):

- Es müssen eindeutig messbare Leistungskriterien zu finden sein.

- Das Ergebnis muss von den betreffenden Mitarbeitern wirklich beeinflussbar sein.

- Damit sich der Aufwand der Arbeitsstudien lohnt und die Mitarbeiter eine normale Leistung erreichen können, muss die Arbeit über längere Zeit gleich bleibend sein. Diese Eigenschaft bezeichnet man als Akkordfähigkeit.

- Der Arbeitsablauf muss akkordreif, d.h. störungs- und mängelfrei sein.

Die Vorzüglichkeit des Akkordlohns wird sich in der Landwirtschaft nicht allzu leicht einstellen, denn die meisten Tätigkeiten in der Landwirtschaft unterliegen 
stochastischen Einflüssen und sind nicht über länger Zeit gleich bleibend. In der Landwirtschaft findet der Akkordlohn daher vor allem in der Gemüse- und Obsternte Anwendung. Vor allem Erntehelfer werden in diesen Bereichen im Akkord bezahlt.

BILLIKOPF (2003: 103) berichtet von einem landwirtschaftlichen Obstanbauer, der nach der Einführung des Geldakkordsystems feststellte, dass die Leistung seiner Mitarbeiter deutlich höher als erwartet ausfiel. Daraufhin beschloss er den Lohn/Stück zu senken. Daraufhin verließen ihn die besten Mitarbeiter und die Motivation der verbleibenden Mitarbeiter nahm beträchtlich ab. Das Beispiel zeigt, dass der Bemessung des Lohns/Stück und der exakten Feststellung der Normalleistung besondere Bedeutung zukommt, denn einmal eingeführte Lohnhöhen pro Arbeitseinheit lassen sich im Nachhinein schwer senken. Die Reaktion der Mitarbeiter ist konsistent mit der theoretischen Bedeutung des Status Quo und der Verlustaversion.

Die Vorteile des Akkordlohns sind die Bindung der Bezahlung an die Leistung, sofern diese wirklich vom Mitarbeiter beeinflussbar ist. Außerdem können die Mitarbeiter zu starken Bemühungen motiviert werden und eine positive Selektion der Mitarbeiter ist ebenfalls möglich.

Die oben genannten Vorteile des Akkordlohns können durch empirische Ergebnisse gestützt werden. LAZEAR (2000b) beschreibt eine Fallstudie aus dem Unternehmen Safelite Glass Corporation, einem Unternehmen, das Autoscheiben herstellt und einbaut. Durch den Übergang zum Akkordsystem ergab sich ein Anstieg der Produktivität um 44 \%. Er besteht nach LAZEAR (2000b: 1347) aus zwei Effekten. Erstens steigt durch die Akkordentlohnung die Produktivität der durchschnittlichen Arbeiter und zweitens werden durch das System fähigere Arbeiter angezogen und hoch produktive Arbeiter verlassen seltener die Firma. Im obengenanten Beispiel erhalten die Arbeiter im Akkordsystem durchschnittlich eine 10 \%ige Erhöhung ihres Lohnes im Vergleich zum Stundenlohnssystem.

Es besteht bei der Akkordentlohnung aber die Gefahr, dass durch den Akkord die Qualität des Produkts leidet (WolfF Und LaZEAR 2001: 274, Scholz 2000: 752). Ist die Produktqualität entscheidend, so kann der Akkordlohn an eine Qualitätsprämie gekoppelt werden. In der Firma Safelite müssen die Arbeiter Qualitätsmängel unbezahlt nachbessern, bevor sie bezahlte Aufträge ausführen dürfen (LAZEAR 2000b: 1358). Der 
Qualitätsmangel äußert sich im oben genannten Beispiel durch gebrochene Autoscheiben, die auf unsachgemäßen Einbau zurückzuführen sind.

Ein weiterer Nachteil des Akkordlohns ist eine mögliche Überforderung der Mitarbeiter durch das Entlohnungssystem. Außerdem kann sich bei dauerhafter Erhöhung der Normalleistung der Akkordlohn zu einem Fixlohn entwickeln, wenn sich die Mitarbeiter mit ihrer Leistung immer an der Obergrenze bewegen (SCHOLZ 2000: 752). In diesem Fall würde vom Akkordlohnsystem kein zusätzlicher Leistungsanreiz mehr ausgehen.

Die nachstehende Abbildung 12 zeigt den Verlauf des Akkordlohns in Euro in Abhängigkeit von der Anzahl der Besamungen nach dem Tarifvertrag der Rinderzucht Schleswig-Holstein (RSH) eG, dessen Entlohnungsregelungen für Außendienstmitarbeiter in Kapitel 2.3.2.1 dargestellt wurden.

\section{ABBILDUNG 12: STUNDEN- VERSUS AKKORDENTLOHNUNG IN DER RINDERZUCHT}

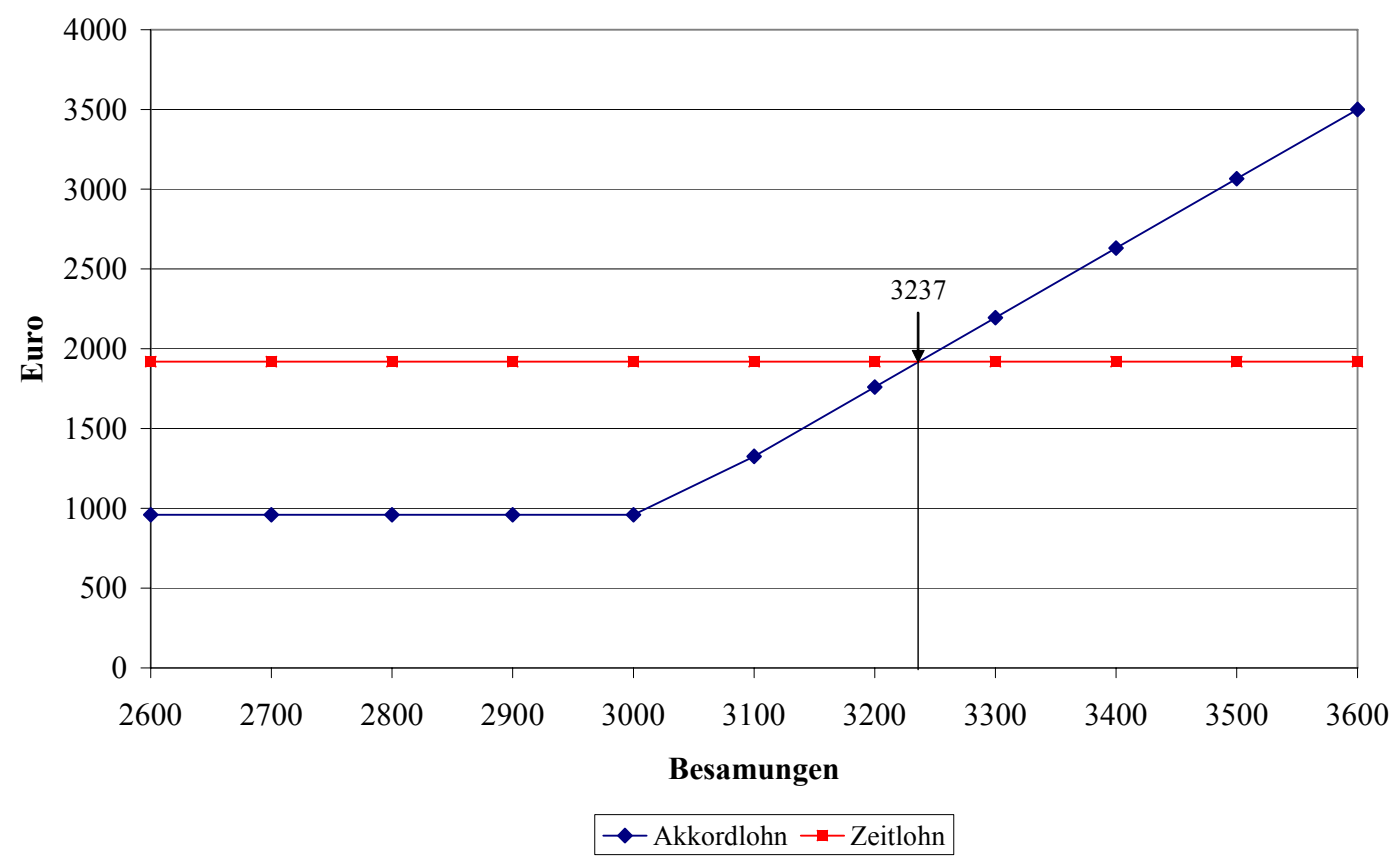

Quelle: Eigene Darstellung, Entgelttarifvertrag für die Beschäftigten der Rinderzucht Schleswig-Holstein eG vom 16.01.2002 und Tarifvertrag für die Vergütung von Außendienstmitarbeitern der Rinderzucht Schleswig-Holstein eG vom 16.06.2003

Im hier angeführten Beispiel setzt sich der Akkordlohn aus einem Grundlohn von 50 \% der Entgeltstufe III (Alterstufe 24. bis 30. Lebensjahr) und einem Akkordzuschlag von 4,35 Euro pro Besamung, der ab der 3016. Besamung greift, zusammen. Zum Vergleich 
ist der Lohn bzw. das Gehalt der entsprechenden Entgeltstufe III dargestellt, den die Außendienstmitarbeiter in der Realität jedoch nicht erhalten, da sie ausschließlich im Akkordsystem arbeiten. Der Tarifvertrag sieht weitere Prämien vor. Diese sind aus Gründen der Vereinfachung hier nicht aufgeführt.

Es wird deutlich, dass ein Unternehmen, das ein Zeitlohnsystem bietet, vor allem für Mitarbeiter attraktiv ist, die weniger als 3.237 Besamungen im Jahr durchführen. Ab dieser Anzahl der Besamungen wird das Arbeiten im Akkordsystem attraktiver. Akkordlohnsysteme haben deshalb einen positiv wirkenden selektiven Charakter in Bezug auf die Leistung der Mitarbeiter. Das Beispiel ist insofern hypothetisch, als dass Mitarbeiter von Rinderzuchtgenossenschaften in einer bestimmten Region in der Regel nicht die Auswahl zwischen verschiedenen Genossenschaften haben.

Die Grafik verdeutlicht außerdem, wie stark die Einkommensmöglichkeiten mit der Anzahl der Besamungen steigen. Eine Obergrenze sieht der Entgelttarifvertrag nicht vor. Die Anzahl der Besamungen wird jedoch alleine durch die Anzahl der Rinderhalter im Bezirk und der technisch möglichen Besamungen (inklusive Anfahrt) beschränkt.

Damit die Qualität der Besamungen nicht leidet, wird ein Besamungsindex von 1,8 als Standard bei der Bemessung des Akkordlohns zugrunde gelegt.

Der fortschreitende Strukturwandel und die Milchleistungssteigerungen in den landwirtschaftlichen Betrieben führen dazu, dass im Zeitablauf die Anzahl der Besamungen im Bereich der RSH für die Besamungstechniker sinken wird. Dadurch wird die Möglichkeit, durch das Leistungslohnsystem ein großes zusätzliches Einkommen zu erzielen, geringer. Dieser Problematik wird im oben genannten Tarifvertrag Rechnung getragen. In Paragraf 11 ist verankert, dass von Seiten der Genossenschaft alles getan wird, um die 3.350 grundgebührenpflichtigen Besamungen pro Tierzuchttechniker zu erhalten.

\subsubsection{Teamvergütungen}

Nicht immer ist es möglich, die Leistung individuell zu messen. Wenn verschiedene Personen die Leistung gemeinsam erbringen oder die Gefahr besteht, dass die Mitarbeiter durch individuelle Leistungsentgelte das Teamergebnis gefährden, können Gruppenanreize ein probates Mittel sein. 
Gruppenanreize funktionieren über die Ausübung von Gruppendruck. Da in manchen Situationen der Arbeitgeber weniger über die individuelle Arbeitsleistung informiert ist als die Teammitglieder, kann beim Gruppenanreiz dieses Problem gelöst werden, indem die einzelnen Teammitglieder auf den Arbeitsbeitrag des Einzelnen achten (MILGROM UND ROBERTS 1992: 416).

Leistungsvergütungen, die sich auf die Gruppe beziehen, haben jedoch den Nachteil, dass sie einen Anreiz zum so genannten „Trittbrettfahren“ schaffen können. Das Trittbrettfahrerverhalten kann durch verschiedene Maßnahmen begrenzt werden. Eine entscheidende Rolle spielt dabei die Teamgröße, in kleinen Teams ist die Gefahr des Trittbrettfahrens geringer als in großen Teams, da die Mitarbeiter den Leistungsbeitrag des Kollegen besser beobachten können. Außerdem ist es wichtig, die Teams so zusammen zu stellen, dass die Mitglieder möglichst ähnliche Tätigkeiten ausüben (BACKES-GELLNER ET AL. 2001: 334-335).

Eine Möglichkeit eine zeitlich begrenzte Teamleistung zu belohnen sind Teamboni. Das Team erhält hierbei einen Bonus für ein zeitlich überschaubares Projekt, um zu vermeiden, dass sich die Teamzusammenstellung während der Projektlaufzeit ändert (ebenda: 335 f.).

Ein Modell zur leistungsorientierten Gruppenvergütung, das in der Milchproduktion in einigen Betrieben zur Anwendung kommt, ist der Vergütungsfonds. Für jedes Kilogramm ermolkener Milch wird ein gewisser Betrag (z.B. 5 Cent) in den Fonds eingezahlt. Die Mitarbeiter in der Milchproduktion werden aus diesem Fonds anteilig für ihre geleisteten Arbeitsstunden entlohnt (SÄCHSISCHE LANDESANSTALT FÜR LANDWIRTSCHAFT 2004: 45, WÜLLMERS 1995: 78).

\subsubsection{Erfolgsbeteiligungen}

Die ökonomische Fragestellung der Entlohnung von Führungskräften wird durch das Prinzipal-Agenten-Problem verdeutlicht, wenn der Manager des Unternehmens nicht mit dem Eigentümer identisch ist.

Im Gegensatz zu Arbeitern in der Produktion haben Führungskräfte einen direkteren Einfluss auf den Unternehmenserfolg. Aus diesem Grund können Kennziffern des Unternehmenserfolgs eher als Basis für eine Zusatzvergütung herangezogen werden. Außerdem können Anreize, die ein hohes Sozialprestige vermitteln, einen höheren 
Stellenwert haben. Hierzu zählen z.B. Dienstwagen, exklusive Büroausstattung oder großzügige Spesenerstattungen (EVERS 1995: 298).

Formen von variablen Bezügen sind Erfolgs- oder Gewinnbeteiligungen, Tantiemen, Prämien oder andere Zusatzvergütungen, wie z.B. zusätzliche Sozialleistungen oder geldwerte Sachleistungen.

Erfolgsbeteiligungen von Führungskräften können in Form von Aktien oder Aktienoptionen gewährt werden. Im Unterschied zu Aktien stellen Aktienoptionen keinen Anspruch auf Beteiligung am laufenden Gewinn des Unternehmens dar, sondern eine Kaufoption zu einem festgelegten Ausübungspreis (BACKES-GELLNER ET AL. 2001: 338 f.).

Da Aktien und Aktienoptionen für die originäre Landwirtschaft aufgrund der untergeordneten Rolle der Aktiengesellschaft keine entscheidende Bedeutung haben, soll als Praxisbeispiel eine Gewinnbeteiligung für Führungskräfte dargestellt werden. Das Leitungspersonal des Agrarunternehmens erhält einen Gehaltzuschlag in Abhängigkeit vom erzielten Gewinn. Der Zuschlag wird in drei Stufen gewährt. Erreichen die Führungskräfte $75 \%, 100 \%$ oder $125 \%$ des geplanten Gewinns, so erhalten sie einen Zuschlag von $10 \%, 20 \%$ oder $30 \%$ (SÄCHSISCHE LANDESANSTALT FÜR LANDWIRTSCHAFT 2002: 48).

Kritiker von Erfolgs- und Gewinnbeteiligungen verweisen auf einen möglichen Fehlanreiz, der sich in kurzfristigen Managemententscheidungen ausdrücken kann. Langfristige Unternehmensziele würden bei dieser Vergütungsform in den Hintergrund treten, da kurzfristige Gewinnentwicklungen für die Führungskräfte monetär entscheidend seien (EISENFÜHR UND THEUVSEN 2004: 218).

Trotz des besonderen Stellenwerts der Erfolgsbeteiligungen ist festzuhalten, dass das Gehalt bei Führungskräften eine zentrale Rolle spielt. Eine Lohnsteigerung ist durch Aufstieg in der Hierarchie möglich, die in Abgrenzung $\mathrm{zu}$ den in Kapitel 4.1.2 dargestellten Vergütungsformen nicht auf Basis des Alters oder der Dauer der Betriebszugehörigkeit geschieht, sondern durch Beförderung.

Erfolgs- und Mitarbeiterbeteiligungen spielen besonders bei der Entlohnung von Führungskräften eine wichtige Rolle. Aber auch Mitarbeiter auf unteren 
Hierarchieebenen können über Erfolgsbeteiligungen entlohnt werden. Die SÄCHSISCHE LANDESANSTALT FÜR LANDWIRTSCHAFT (2004: 45) zeigt ein solches Vergütungsbeispiel für ein landwirtschaftliches Unternehmen auf. In dem Agrarunternehmen erhalten alle Mitarbeiter zu einem Grundlohn eine Gewinnbeteiligung. $35 \%$ des Gewinns werden auf die vorhandenen Mitarbeiter entsprechend ihres Anteils an der Gesamtvergütung verteilt.

Es ist aber zu bedenken, dass diese Mitarbeiter einen deutlich geringeren Einfluss auf das Betriebsergebnis haben und sich deshalb die Frage stellt, wie gerecht eine solche Gruppenvergütung ist. Für das Betriebsergebnis sind neben den marktgegebenen Einflüssen vor allem die Entscheidungen der Unternehmensleitung von Bedeutung. Strategische Entscheidungen werden von den Mitarbeitern in der Produktion in der Regel nicht getroffen. Eine Gewinnbeteiligung kann für diese Gruppe der Beschäftigten zwar einen Anreiz bieten sich überdurchschnittlich für den Betrieb einzusetzen, in schlechten Wirtschaftsjahren oder bei falschen Betriebsleiterentscheidungen, die sich durch einen niedrigen Gewinn ausdrücken, erhalten die Mitarbeiter nur einen reduzierten Lohn im Vergleich zu Jahren mit hohen Gewinnen. Dies kann zu Ungerechtigkeitsempfinden in der Belegschaft führen, da die individuelle Arbeitsleistung der einzelnen Mitarbeiter in wirtschaftlich schlechten Jahren nicht schlechter sein muss als in guten Jahren. Dem Modell von PORTER UND LAWLER folgend, würde dies zu einem Motivationsrückgang führen, da die wahrgenommene Wahrscheinlichkeit, dass eine unternommene Anstrengung auch die ausgelobte Belohnung nach sich zieht, sinkt (vgl. Kapitel 3.2.4).

\subsubsection{Turnierentlohnung}

Die Turnierentlohnung (oder Rank-Order-Tournament) ist eine relative Entlohnungsform. Die Mitarbeiter werden bei der Turnierentlohnung nach ihrer relativen Leistung in eine Rangfolge gebracht und entlohnt.

Für diese Form der leistungsorientierten Entlohnung ist es daher nicht notwendig, die Leistung kardinal zu messen. Die Leistung wird durch ordinale Messung, nämlich durch Einstufung in Rangreihen, bestimmt. Dies unterscheidet die Turnierentlohnung von der relativen Leistungsbewertung, wo neben dem Rang der Wettbewerber auch der Leistungsabstand zwischen den Teilnehmern in die Entgelthöhe einfließt (BUDDE UND WIELENBERG 1997: 924 f., KNOEBER UND THURMAN 1994: 155). 
Die Turnierentlohnung hat dann Vorteile, wenn die Leistung nicht kardinal messbar ist und stochastische Einflüsse den Output beeinflussen. Außerdem ist sie vorteilhaft, wenn noch keine Erfahrungen über sinnvolle Werte zur Entlohnung des Outputs fehlen.

Mit der Turnierentlohnung sind jedoch auch einige mögliche Nachteile verbunden. Es ist zum Beispiel möglich, dass sich die Wettbewerber untereinander absprechen. Außerdem hat sie dort Nachteile, wo ein Teamverhalten für die Produktion des Outputs notwendig ist, denn Turnierentlohnung unterdrückt vollständig Teamproduktion (BUDDE UND WIELENBERG 1997: 943).

Die Anreizwirkung von Turnierentlohnungen ist ferner vom Informationsstand der Wettbewerber bezüglich ihrer Leistungsfähigkeit abhängig. Handelt es sich um gleich leistungsfähige Mitarbeiter, so ist von einer positiven Anreizwirkung eher auszugehen als bei heterogenen Leistungspotenzialen der Mitarbeiter. Ist die Leistung der Mitarbeiter nämlich heterogen und sind die Leistungsunterschiede den Mitarbeitern bekannt, so führt dies zu einer verminderten Leistungsbereitschaft der leistungsstarken und leistungsschwachen Mitarbeiter. Die leistungsstarken Mitarbeiter brauchen nur eine geringere Anstrengung aufbringen als im Wettbewerb mit gleich starken Wettbewerbern. Die leistungsschwachen Mitarbeiter hingegen bemühen sich weniger, da sie davon ausgehen, dass sie den Wettbewerb ohnehin nicht gewinnen können (KNOEBER UND THURMAN 1994: 157).

KNOEBER UND THURMAN schlagen zwei Maßnahmen vor, um die Anreizwirkung bei vollständiger Information heterogenen Wettbewerber über die Fähigkeiten der Mitspieler zu sichern. Organisatoren von Turnieren können weitere Spieler beauftragen, am Spiel teilzunehmen oder ein Handicap-System einführen, das dem schlechteren Wettbewerber einen Vorsprung einräumt (ebenda: 170).

Turnierentlohnungen erhöhen - wie Stücklohnsysteme übrigens auch - Durchschnitt und Varianz der Leistungen. Heterogene Spieler unterscheiden sich auch in der Risikofreudigkeit ihrer Aktivitäten. Die fähigeren Spieler verhalten sich eher risikoavers und vollbringen Leistungen mit geringerer Varianz und die schwächeren Mitbewerber verhalten sich eher risikofreudig, um durch riskante Aktionen ihre Chancen auf einen Gewinn des Turniers zu erhöhen. 
Ein Beispiel für Turnierentlohnung stellen Beförderungen dar, da Mitarbeiter in Abhängigkeit von ihrer relativen Leistung in der Hierarchie aufsteigen. Weitere Beispiele finden sich in der Zulieferindustrie von Automobilherstellern oder bei Forschungs- und Entwicklungswettbewerben (BUDDE UND WIELENBERG 1997: 923).

KNOEBER UND THURMAN (1994) testen zwei verschiedene Entlohnungssysteme in der Vertragsproduktion von Mastbroilern. In einem Fall handelt sich um ein Entlohnungssystem des Turniertyps und im zweiten Fall um ein Vergütungssystem mit einer linearen relativen Leistungsbewertung. Die Leistung des Mästers bemisst sich in beiden Entlohnungstypen anhand der Mastkosten je produziertes Mastgewicht. Im Turniermodell werden die Mäster nach ihrer Rangfolge in der Höhe der Mastkosten pro Pfund bezahlt. Im Modell der relativen Leistungsbewertung wird die Entlohnung nach dem Abstand zum durchschnittlichen Mäster bestimmt.

In den beobachteten Fällen der Mastbroilerproduktion gibt es zwei Parteien. Der Integrator ist eine Hähnchen verkaufende Firma, die dem Mäster (Grower) Küken, Futter, tierärztliche Leistungen und Beratungsdienst zur Verfügung stellt. Alle Mäster erhalten das gleiche „Ausgangsmaterial“ und Service. Der Mäster zieht die Küken zum Mastbroiler auf und stellt Unterbringung und Arbeitskraft. Der Integrator „erntet“ und verkauft die ausgemästeten Broiler.

Im Wettbewerb befinden sich alle Mäster des Integrators, die im Zeitraum von 1-2 Wochen die Broiler „ernten“. Die Leistung des Mästers besteht im Wesentlichen in einer guten Futterumsetzung der Tiere, geringen Mortalitätsraten und schweren Tieren, die die Höhe der Mastkosten bestimmen (KNOEBER Und ThURMan 1995: 487). Die Entlohnung nach Turnier oder relativer Leistungsbewertung eignet sich in diesem Fall, da zwar stochastische Einflüsse die Produktion beeinflussen, diese jedoch alle Mäster im gleichen Maße betreffen (KNOEBER UND THURMAN 1994: 162).

Die Autoren konnten hinsichtlich der theoretischen Vorhersagen zur Turnierentlohnung feststellen, dass die Leistung der Mäster vom Entlohnungsabstand zwischen den Wettbewerbern abhängt und nicht von der absoluten Höhe der Entlohnung - sofern der Lohnabstand zwischen Gewinnern und Verlierern gleich bleibt. Die Implikation für Arbeitgeber, die die Turnierentlohnung anwenden, ist, dass ein Anreiz zur Leistungssteigerung nur über eine Erhöhung des Lohnabstands zwischen Gewinnern und Verlierern gesetzt werden kann. Diesen Sachverhalt verdeutlicht Abbildung 13. 
ABBILDUNG 13: BESTIMMUNG DER ARBEITSANSTRENGUNG BEI DER TURNIERENTLOHNUNG

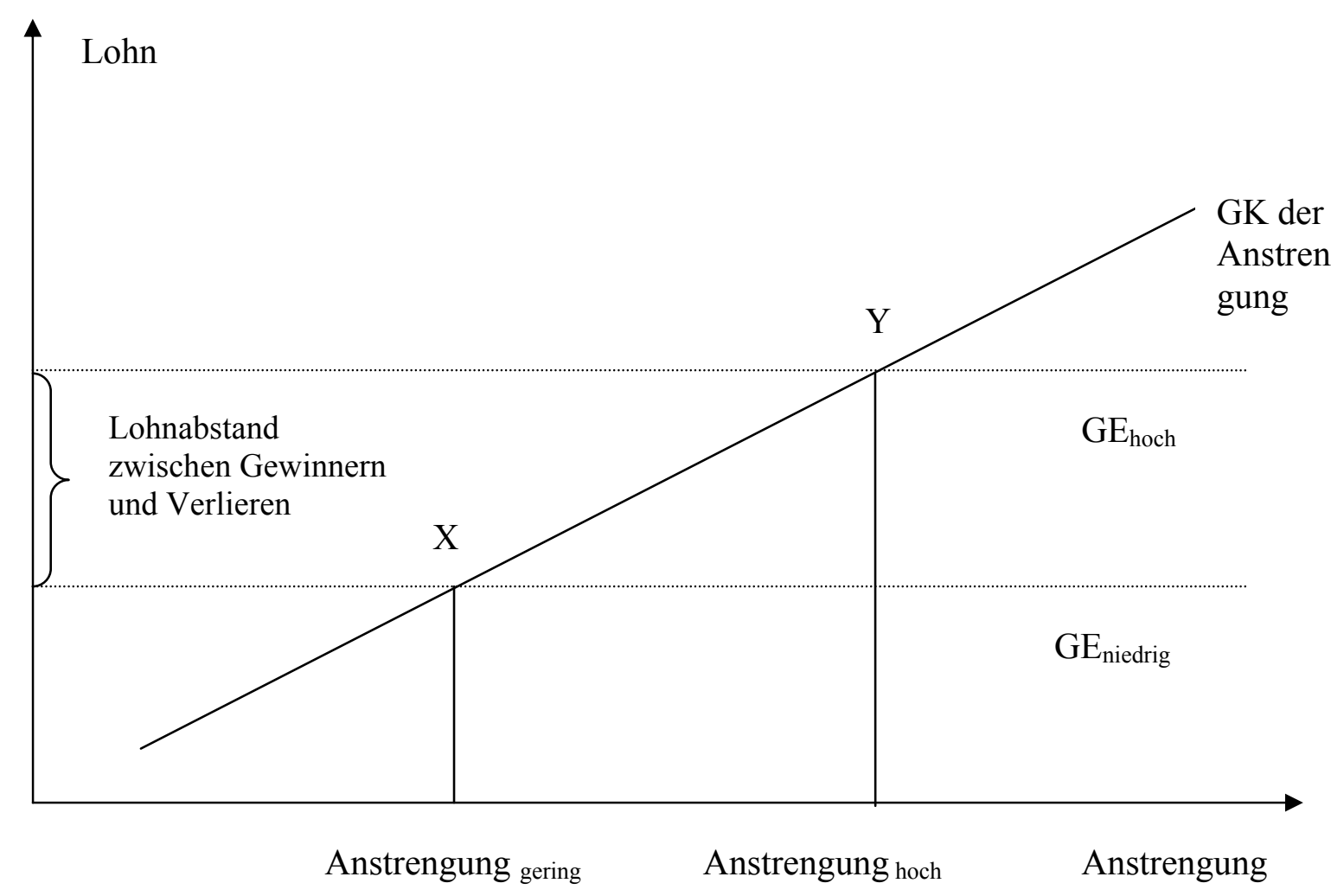

GK der Anstrengung: Grenzkosten der Anstrengung, Arbeitsleid

GE: Grenzerlös des Gewinnens

Quelle: Eigene Darstellung in Anlehnung an Vorlesungsunterlagen von SHELDON 2007

Der Nutzen maximierende Wettbewerber wird nur so viel Arbeitsanstrengung aufbringen, bis der Grenzerlös des Gewinnens gleich den Grenzkosten seines Bemühens ist. Die Grenzkosten der Anstrengung können auch als Arbeitsleid bezeichnet werden. Ist der ausgelobte Preis des Gewinnens gering, so wird ein Wettbewerber ein niedriges Anstrengungsniveau wählen (verdeutlicht durch den Punkt X). Bei einem hohen Grenzerlös des Gewinnens wird sein Anstrengungsniveau steigen (Punkt Y). Wie hoch die Anstrengung ausfällt, hängt außerdem vom Verlauf der Grenzkostenkurve und vom Ausmaß der Unsicherheit ab. Verläuft die Grenzkostenkurve oberhalb der in Abbildung 13 dargestellten Grenzkostenkurve, so muss der Arbeitgeber einen höheren Preis für den Gewinner ausloben. Unterliegt der Output größeren Schwankungen, so muss sich ebenfalls das Gewinnen des Turniers mehr lohnen, da es für die Mitarbeiter schwieriger wird abzuschätzen, ob sich die Bemühung lohnt. Da also die Wahrscheinlichkeit das Turnier durch die individuelle Arbeitsleistung zu gewinnen bei zunehmender 
Unsicherheit sinkt, muss die Lohnspreizung zwischen Gewinnern und Verlieren steigen (BACKES-GELLNER ET AL. 2001: 173).

KNOEBER UND THURMAN (1994: 178) konnten außerdem anhand ihres Datenmaterials zeigen, dass sich die Varianz der Leistung bei den Turnierteilnehmern bei heterogener Wettbewerberstruktur unterscheidet. Die leistungsschwächeren Mäster zeigten höhere Varianzen in der Leistung als die Leistungsstärkeren. Ineffizienzen in gemischten Wettbewerbssituationen werden dadurch vermieden, dass immer die Mäster im Wettbewerb stehen, die ähnlich lange Produktionsperioden haben. Die Länge der Produktionsperiode ist Ausdruck für die Arbeitsleistung der Mäster, da die erfolgsreichen Mäster kürzere Produktionszyklen als die leistungsschwächeren Mäster haben.

Zwar wird ein Mäster schon durch kürzere Mastzyklen zusätzlich entlohnt, die Turnierentlohnung bietet aber sowohl dem leistungsstärkeren als auch dem -schwächeren einen Anreiz, die Mastkosten weiter zu senken. Dadurch sinken die durchschnittlichen Mastkosten für den Integrator insgesamt.

Die Turnierentlohnung hat einen Nebeneffekt. Das Preisrisiko wird vom Mäster an den Integrator übertragen, da für die Entlohnung der Mäster nur die relative Höhe der Mastkosten eine Rolle spielt. Die Firmen, die die fertigen Broiler vermarkten, verfügen über ein großes Portfolio an Produkten, wodurch ihr Risiko diversifiziert wird. Für den Integrator ist die Übernahme des Preisrisikos kein Nachteil, da durch diesen Mechanismus das Angebot an Broilern konstant bleibt (KNOEBER UND THURMAN 1994).

\subsection{Cafeteria-Systeme als individualisierte Vergütungsform}

Cafeteria-Systeme sind ein Versuch, die Personalentlohnung zu individualisieren. Die Mitarbeiter haben die Möglichkeit, sich ihre Entlohnung wie in einem Menü im Rahmen eines vorgegebenen Budgets selbst zusammenzustellen.

Das Unternehmen bestimmt, aus welchen Entgeltkomponenten die Mitarbeiter auswählen können. Neben materiellen Leistungen wie Versicherungen (z.B. zur Altersvorsorge), Beteiligungen und Sachleistungen können auch immaterielle Komponenten gewählt werden. Hierzu gehört zum Beispiel die Individualisierung der Arbeitszeit bezogen auf die Perioden- bzw. Jahresarbeitszeit (SCHOLZ 2000: 754 f.). 
Die einzelnen Komponenten müssen mit einer Wertigkeit belegt werden, mit der sie in das Gesamtbudget einfließen. Außerdem muss die Dauer der Periode festgelegt werden, für die die Zusammenstellung der Komponenten gilt, da sich die Bedürfnisse der Mitarbeiter im Zeitablauf ändern können. Abschließend ist zu regeln, wie vorgegangen werden soll, wenn ein Mitarbeiter das Budget in der Abrechnungsperiode nicht ausschöpft.

Die Vorteile von Cafeteria-Systemen liegen in der Anpassung des Entgeltsystems an die individuellen Bedürfnisse der Mitarbeiter. Ein weiterer Vorteil ist, dass die Entlohnungsmöglichkeiten für die Mitarbeiter transparenter werden, da sie für die Komponentenwahl offen gelegt werden. Oft ist den Mitarbeitern nicht bekannt, welche Wahlmöglichkeiten das Unternehmen überhaupt anbieten kann. Außerdem kann das Bewusstsein für die Kosten, die verschiedene Optionen verursachen, bei den Mitarbeitern geschärft werden (BITSCH 2003: 104).

Nachteile des Cafeteria-Systems sind die damit verbundenen Administrationskosten und die Planung hinsichtlich der gewählten Arbeitszeitvereinbarungen (ebenda: 104).

Cafeteria-Systeme könnten auch in der Landwirtschaft Anwendung finden, da sie den Vorteil haben, dass sie den individuellen Präferenzen der Mitarbeiter besser gerecht werden als einheitliche Entlohnungs- oder immaterielle Anreizsysteme. Da die Mitarbeiter in den landwirtschaftlichen Betrieben keine homogene Gruppe hinsichtlich des Alters (vgl. hierzu die Ausführungen in Kapitel 2.2.1) oder des Familienstandes sind, werden auch die Bedürfnisse hinsichtlich des Bedarfs an Lohn, Freizeit oder zusätzlichen Sozialleistungen unterschiedlich sein. Zurückgreifend auf Maslows Bedürfnishierarchie haben individualisierte Anreizsysteme den Vorteil, dass sich der Mitarbeiter aus einem - wenn auch begrenzten - Pool an Anreizsystemen, das für ihn bedeutsamste aussuchen kann. Der Betriebsleiter muss also nicht selbst erahnen, wie er den einzelnen Mitarbeiter am besten motivieren kann. Die Grenzen der Anwendbarkeit von Cafeteria-Systemen in der Landwirtschaft liegen zum einen im Arbeitsaufwand für die Einrichtung des Systems mit den dazugehörigen Menükomponenten. Aufgrund der oftmals geringen Anzahl von Lohnarbeitskräften pro Betrieb können Kosten und möglicher Nutzen des Systems in einem unausgewogenen Verhältnis stehen. Zum zweiten sind der Flexibilisierung der Arbeitszeiten als eine wichtige Komponente von Cafeteria-Systemen durch die Besonderheiten der Landwirtschaft enge Grenzen gesetzt. 
In den Arbeitsspitzen, wie z.B. der Getreideernte, wird es den Mitarbeitern nicht möglich sein, frei zu nehmen. Hinzukommt, dass die Anzahl der Mitarbeiter oft nicht ausreichen wird, um z.B. attraktive Konditionen bei Versicherungsunternehmen aushandeln zu können, wie dies Arbeitgeber mit vielen Beschäftigten möglich ist.

Das Kapitel 4 zeigt, dass es zahlreiche materielle Anreizsysteme gibt, deren Vorzüglichkeit von den gegebenen Rahmenbedingungen abhängt, unter denen sie Anwendung finden. Während die inputabhängigen Vergütungssysteme wie der Zeitlohn oder die Anpassung der Lohnhöhe an das Alter oder den Sozialstatus in der Landwirtschaft ohne große Schwierigkeiten angewendet werden können und zum Großteil auch in den vorhandenen Tarifverträgen institutionalisiert sind, begrenzen die dargestellten Besonderheiten der landwirtschaftlichen Produktion (vgl. 2.1) und die sich aus den theoretischen Ansätzen ergebenden Erfolgsbedingungen leistungsorientierter Entlohnung zum Teil die Einsatzmöglichkeiten einiger outputabhängiger Entgeltformen. So ist z.B. die Akkordfähigkeit eine wichtige Voraussetzung für die Entlohnung nach Akkord, eine Bedingung, die nur vereinzelt (z.B. in der Obst- und Gemüseernte) in der Landwirtschaft vorzufinden ist. Die im nächsten Kapitel vorgestellte empirische Analyse wird die relative Bedeutung der in Kapitel 4 vorgestellten materiellen Anreizsysteme zeigen. 


\section{Empirische Analyse zur leistungsorientierten Entlohnung in der Landwirtschaft}

In diesem Kapitel werden Konzeption, Durchführung und Ergebnisse der empirischen Analyse zur leistungsabhängigen Lohngestaltung in der Landwirtschaft vorgestellt.

\subsection{Methodik und Durchführung der Befragungen}

Das Thema der Arbeit ist ein wenig bearbeitetes Themenfeld in der deutschen agrarökonomischen Forschung. Wenngleich einzelne Anwendungsgebiete leistungsorientierter Entlohnung in der Landwirtschaft in vorangegangenen Kapiteln angedeutet wurden, muss aus diesem Grund zunächst der Status Quo der Gestaltung von Anreizsystemen in den landwirtschaftlichen Betrieben Deutschlands im Allgemeinen festgestellt werden. Damit besteht die Möglichkeit, eine umfassende Analyse für die Landwirtschaft vorzunehmen und daraus Empfehlungen für die Praxis abzuleiten.

Bisherige Analysen zur Gestaltung von Anreizsystemen in der Landwirtschaft konzentrieren sich auf die neuen Bundesländer (z.B. DoLUSCHITZ ET AL. 1996, BECKMANN 2000, WüLlmERS 1995). Deshalb war ein Ziel, sowohl in den neuen als auch in den alten Bundesländern Betriebsleiter zu befragen, um auch die Perspektive der landwirtschaftlichen Arbeitgeber im früheren Bundesgebiet in die empirische Analyse aufnehmen und mögliche Unterschiede analysieren zu können.

Außerdem sollten in der Befragung die unterschiedlichen Rechtsformen, Wirtschaftweisen (ökologisch versus konventionell) und betriebswirtschaftlichen Ausrichtungen in der empirischen Analyse vertreten sein. Um auch multivariate Analysemethoden anwenden zu können, wurden schriftliche Befragungen konzipiert, da hierfür eine Stichprobe von mindestens 100 Betrieben angestrebt wurde. Diese bildet den Schwerpunkt der empirischen Analyse.

Die Arbeitgeberbefragung wurde im Zeitraum von Juli 2005 bis März 2006 in zwei Erhebungsphasen durchgeführt. Der Fragebogen für die Arbeitgeber wurde in einem Pre-Test im Face-to-face-Interview mit Landwirten auf Verständlichkeit, Vollständigkeit und Bearbeitungsdauer getestet. Der daraufhin modifizierte Fragebogen wurde von insgesamt 260 Landwirten verschiedener betriebswirtschaftlicher Ausrichtungen in unterschiedlichen Regionen Deutschlands beantwortet. 
Um außerdem einen Einblick in die Einstellung von landwirtschaftlichen Lohnarbeitskräften zu verschiedenen Anreizsystemen und zu ihren Erfahrungen mit leistungsorientierten Vergütungsformen $\mathrm{zu}$ erhalten, wurde zusätzlich eine Mitarbeiterbefragung konzipiert. Aufgrund der Sensibilität des Themas und um soziale Gewünschtheit bei den Antworten auszuschließen, wurde auf eine mündliche Befragung verzichtet und ein schriftlicher Kurzfragebogen entworfen.

Die Mitarbeiterbefragung wurde im Januar und Februar 2006 im Rahmen von Arbeitnehmerschulungen zum Thema „Vom Landwirt zum Energiewirt“ durch die Landwirtschaftskammer Hannover durchgeführt. Im Vergleich zur Betriebsleiterbefragung ist der Fragenbogen (vgl. Tabellenband im Anhang) für die Mitarbeiter deutlich kürzer, um die Beantwortung im Rahmen der Schulung zu erlauben. Dieser Fragebogen wurde von insgesamt 124 Teilnehmern beantwortet. Im Vorfeld der Befragung wurde der Fragebogen auf Verständlichkeit und Bearbeitungsdauer im Rahmen der Meisterkurse, die ebenfalls von der Landwirtschaftskammer Hannover geleitet werden, getestet. Damit konnte im Vorfeld die Befragungssituation geprüft werden.

Die Ergebnisse der Befragungen wurden nach Abschluss der Befragung mit Betriebsleitern und Lohnarbeitskräften diskutiert. Für die Arbeitnehmer wurden fünf Veranstaltungen zur Diskussion der Ergebnisse durchgeführt. Diese fanden im gleichen institutionellen Rahmen wie die schriftliche Befragung statt. Die Arbeitnehmerschulungen der Landwirtschaftskammer Niedersachsen ${ }^{17}$ finden jährlich im Winter statt. Der Teilnehmerkreis setzt sich in jedem Jahr überwiegend aus den gleichen Personen zusammen, so dass an den Ergebnisworkshops zum großen Teil die Personen teilnahmen, die im Jahr zuvor auch an der schriftlichen Befragung teilgenommen hatten.

Die Diskussion der Ergebnisse mit den Betriebsleitern fand in Vorträgen und Experteninterviews statt. Auch mit Vertretern der landwirtschaftlichen Beratungsinstitutionen wurden die Ergebnisse diskutiert.

17 Die Landwirtschaftskammer Niedersachsen ist im Jahre 2006 aus der Fusion der Landwirtschaftskammern Hannover und Weser-Ems hervorgegangen. Im Mitarbeiterfragebogen, der im Anhang abgedruckt ist, ist noch die alte Bezeichnung Landwirtschaftskammer Hannover zu finden, da die schriftliche Befragung vor der Fusion stattgefunden hat. 


\subsubsection{Arbeitshypothesen und Untersuchungsmodell der Befragungen}

In der folgenden Abbildung 14 ist das Untersuchungsmodell aufgeführt, auf dem der Fragebogen basiert.

ABBILDUNG 14: UNTERSUCHUNGSMODELL DER EMPIRISCHEN ANALYSE

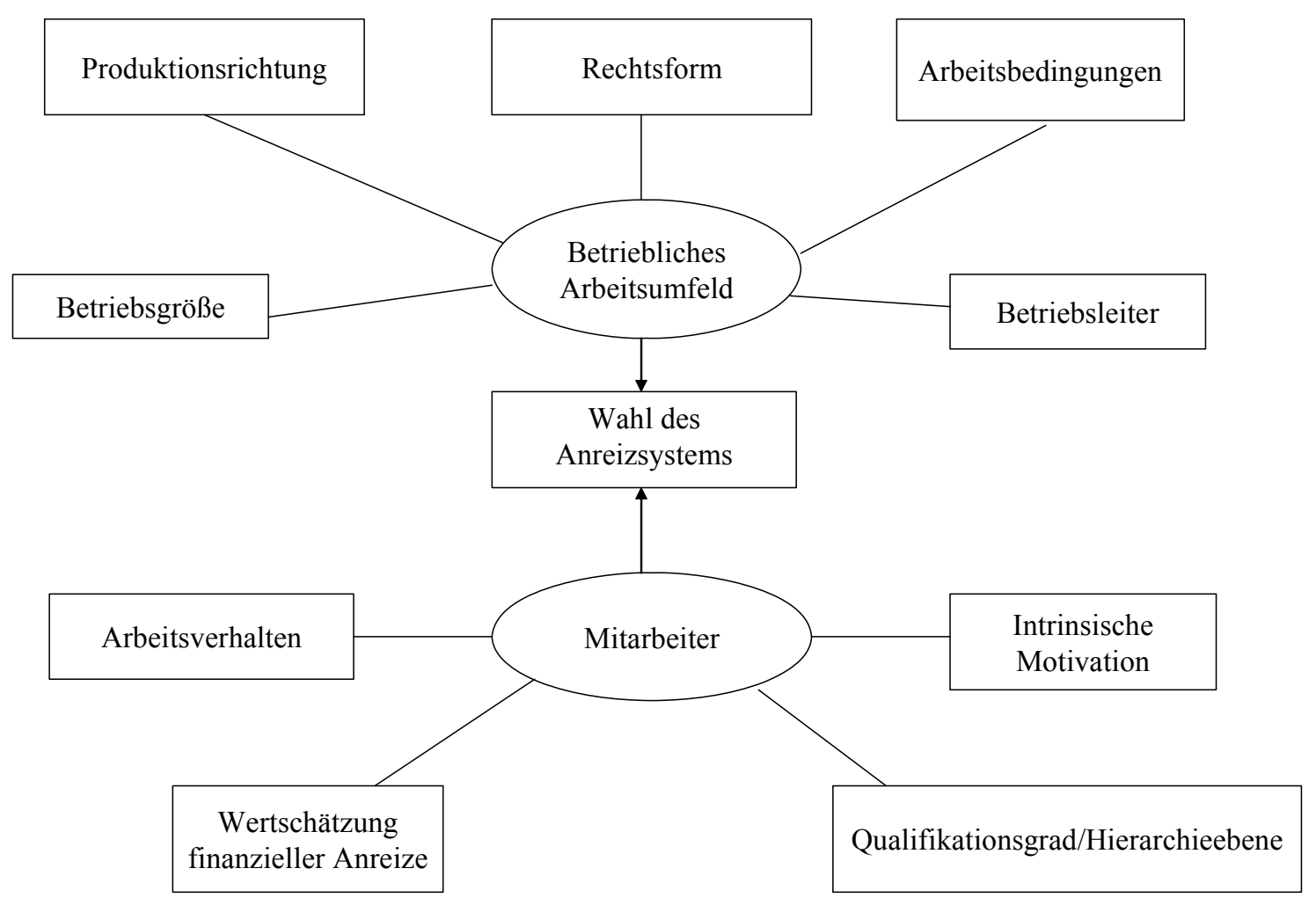

Quelle: Eigene Darstellung

Die Darstellung der verschiedenen theoretischen Ansätze hat gezeigt, dass die Eignung und Wahl eines Anreizsystems von verschiedenen Faktoren beeinflusst wird.

Einige dieser Faktoren lassen sich als betriebliches Arbeitsumfeld zusammenfassen. Hierzu zählen die Produktionsrichtung in der das Anreizsystem angewendet wird, die Rechtsform und die Arbeitsbedingungen im weiteren Sinne. $\mathrm{Zu}$ den Arbeitsbedingungen zählt zum Beispiel die Messbarkeit der individuellen Arbeitsleistung, die u.a. davon abhängt, ob es sich um ein Team oder eine Einzelperson handelt oder ob stochastische Einflüsse wie das Wetter auf das Produktionsergebnis einwirken. Zum Arbeitsumfeld gehören außerdem die Betriebsgröße und die Betriebsleitereigenschaften, wie zum Beispiel die Kommunikation der angestrebten Unternehmensziele gegenüber den Mitarbeitern. 
Verhaltenswissenschaftliche Ansätze sehen den Mitarbeiter und seine individuellen Präferenzen, Rollenwahrnehmung und Fähigkeiten als eine wesentliche Einflussgröße bei der Wahl des Anreizsystems. Im Rahmen der Arbeitgeberbefragung wurden deshalb Items zur intrinsischen Motivation, zum Arbeitsverhalten und zur Wertschätzung finanzieller Anreize der Mitarbeiter formuliert. In der Mitarbeiterbefragung wurden Fragenkomplexe zur intrinsischen Motivation und zum Risikoverhalten aufgenommen.

Im Folgenden werden die Hypothesen aufgeführt und erläutert, die sich aus den in Kapitel 3 dargestellten theoretischen Hintergründen ableiten und den beiden Fragebögen zugrunde liegen.

Hypothesen zur Einflussgröße „,Betriebliches Arbeitsumfeld“

H1: Leistungsorientierte Entlohnungssysteme spielen in der Tierproduktion eine größere Rolle als im Ackerbau.

Die Besonderheiten landwirtschaftlicher Produktionsprozesse führen dazu, dass stochastische Größen einen Einfluss auf die vollbrachte Arbeitsleistung haben (KNOEBER Und ThuRman 1995: 486). Durch das große Zeitfenster („time lag“) zwischen Arbeitseinsatz und Erfolg im Ackerbau, dem Eingreifen verschiedener Personen in den Produktionsprozess sowie dem Einfluss der Witterung ist davon auszugehen, dass leistungsorientierte Entlohnungssysteme im Ackerbau eine untergeordnete Rolle spielen, da die Arbeitsleistung des Einzelnen kaum messbar ist. Außerdem erleichtern es Computer gestützte Herdenmanagementprogramme in der Tierproduktion, Messkriterien für Leistungslöhne zu bestimmen, die mit einem geringen zusätzlichen Kontrollaufwand verbunden sind.

H2: In den neuen Bundesländern finden leistungsorientierte Entlohnungssysteme häufiger Anwendung als in den alten Bundesländern.

Normenkataloge zur Bemessung leistungsabhängiger Einzel- und Kollektivvergütungen spielten in der Landwirtschaft der ehemaligen DDR eine wichtige Rolle (GABLER 1995: $161 \mathrm{ff}$.). Es ist $\mathrm{zu}$ erwarten, dass zumindest in den LPG-Nachfolgeunternehmen leistungsorientierte Entgeltgestaltungen eine überdurchschnittliche Bedeutung aufweisen. 
H3: Je mehr Mitarbeiter beschäftigt sind, desto höher ist die Wahrscheinlichkeit des Einsatzes leistungsorientierter Entgeltsysteme.

Das Prinzipal-Agenten-Problem tritt besonders dann auf, wenn der Arbeitseinsatz schwer zu beobachten ist. Dies wird durch eine steigende Anzahl beschäftigter Personen begünstigt. Es wird daher angenommen, dass der Einsatz leistungs- oder erfolgsorientierter Entlohnung mit der Anzahl der Beschäftigten steigt.

\section{Hypothesen zur Einflussgröße „,Mitarbeiter“}

H4: Die Mitarbeiter in der Landwirtschaft schätzen ihren Arbeitsplatz.

In Kapitel 3 wurde deutlich, dass die verschiedenen ökonomischen Theorien ein kontroverses Menschenbild beinhalten. Die Prinzipal-Agenten-Theorie geht davon aus, dass Mitarbeiter „Arbeitsleid“ empfinden und deshalb zum Bummeln geneigt sind (Odening 1994: 272, LAUX UND LIERMANN 2003: 526 ff., Jost 2001: 17 ff.). Demgegenüber stehen die Annahmen der verhaltenswissenschaftlich geprägten Ökonomen, die tendenziell darauf verweisen, dass Menschen nicht immer eigennützig handeln und den Arbeitsinhalt hoch schätzen können (FREY ET AL. 2001: 564). Die von KROMKA (2004: 82) dargestellten empirischen Ergebnisse zeigten, dass Landwirte trotz hoher Arbeitsbelastung und schlechter Bezahlung im Vergleich $\mathrm{zu}$ anderen Berufsgruppen (vgl. Kapitel 2.1) eine überdurchschnittliche Zufriedenheit mit ihrer Tätigkeit zeigen. In der Hypothese wird angenommen, dass das mit landwirtschaftlichen Tätigkeiten verbundene „Flow-Erlebnis“ (ebenda: 82 ff.) auch von den mit einfacheren Arbeiten betrauten Mitarbeitern wahrgenommen wird und sich nicht auf Betriebsleiter beschränkt, wie KROMKA postuliert.

H5: Die Wertschätzung finanzieller Anreize ist bei den Arbeitnehmern in der Landwirtschaft hoch.

Zu den Erfolgsbedingungen leistungsorientierter Entlohnung gehört die Wertschätzung finanzieller Anreize durch die Mitarbeiter (ThEuvsen 2003: 487). Diese ist dann besonders hoch, wenn die Mitarbeiter über ein vergleichsweise geringes Gehalt verfügen. Verglichen mit anderen Wirtschaftsbereichen ist die Bezahlung in der Landwirtschaft gering. Folglich ist davon auszugehen, dass die Mitarbeiter in der 
Landwirtschaft finanzielle Anreize, die eine zusätzliche Entlohnung zu einem den Lebensunterhalt sichernden Grundlohn darstellen, hoch schätzen.

H6: Die Hierarchieebene der Mitarbeiter hat einen Einfluss auf die Wahl des Anreizsystems.

Leistungsorientierte Entlohnungssysteme sind vor allem dann sinnvoll, wenn die Mitarbeiter das Arbeitsergebnis auch wirklich beeinflussen können. Dies trifft für die Mitarbeiter zu, denen eigene Verantwortungsbereiche in der Produktion zugeteilt sind. Führungsaufgaben sind hingegen nur schwer messbar, wie Kapitel 4 zeigte, und deshalb kaum mit Hilfe von Leistungslohnsystemen zu entlohnen. Da die Leistung des Führungspersonals sich jedoch unmittelbar im Betriebsergebnis niederschlägt, sind Erfolgsbeteiligungen wahrscheinlicher als auf der Ebene der Mitarbeiter in der Produktion.

\subsubsection{Fragebogenaufbau}

Aufgrund des explorativen Charakters der Befragung wurden der Betriebsleiter- und der Mitarbeiterfragebogen thematisch recht breit angelegt, sie umfassen sowohl materielle als auch immaterielle Anreizsysteme. Der Fragebogenaufbau und die abgefragten Variablen sind in der folgenden Tabelle 11 dargestellt. Die beiden verwendeten Fragebögen sind im Anhang abgedruckt.

Ein Teil der Variablen beschreibt die strukturellen Merkmale der Betriebe, um bei der Auswertung den Einfluss betriebsstruktureller und -organisatorischer Unterschiede auf die Gestaltung von Anreizsystemen bestimmen zu können. Aus diesem Fragenkomplex wurden nur einige wesentliche Kennziffern wie die Betriebsgröße und die Anzahl der Arbeitskräfte in den Mitarbeiterfragebogen übernommen.

Der Unternehmerpersönlichkeit, der individuellen Einschätzung der Wettbewerbsfähigkeit und den Arbeitsschwerpunkten des Betriebsleiters ist ein weiterer Fragebogenabschnitt gewidmet. Die Variablen zu den beiden ersten Punkten wurden als 
fünfstufige Likertskala ${ }^{18}$ erhoben. Zu diesem Abschnitt gehört auch die Einschätzung des zukünftigen Fachkräftebedarfs, die bereits in Kapitel 2 dargestellt wurde. Der Arbeitnehmerfragebogen enthält Fragen zum Risikoverhalten als wichtigem Persönlichkeitsmerkmal, das die Wirkung von Anreizsystemen beeinflussen kann.

TABELLE 11: FragebogenaufBaU DER BETRIEBSLEITER- UND MitARBEITERBEFRAGUNG

\begin{tabular}{|c|c|c|c|}
\hline Fragenschwerpunkt & Erhobene Merkmale & $A G$ & $A N$ \\
\hline $\begin{array}{l}\text { Betriebsstruktur und } \\
\text { Organisationsform }\end{array}$ & $\begin{array}{l}\text {-Flächenausstattung des Betriebes } \\
\text {-Tierbestände } \\
\text {-Anzahl der Arbeitskräfte } \\
\text {-Art der Betriebsberatung } \\
\text {-Rechtsform } \\
\text {-betriebswirtschaftliche Ausrichtung } \\
\text {-Bewirtschaftungsweise } \\
\text {-Hofnachfolge }\end{array}$ & $\begin{array}{l}\mathrm{x} \\
\mathrm{x} \\
\mathrm{x} \\
\mathrm{x} \\
\mathrm{x} \\
\mathrm{x} \\
\mathrm{x} \\
\mathrm{x}\end{array}$ & $\begin{array}{l}\mathrm{X} \\
\mathrm{X} \\
\mathrm{X}\end{array}$ \\
\hline $\begin{array}{l}\text { Persönliche Eigenschaften } \\
\text { und Arbeitsschwerpunkte }\end{array}$ & $\begin{array}{l}\text {-Unternehmerische Ausrichtung } \\
\text {-Risikoneigung } \\
\text {-Einschätzung der betrieblichen } \\
\text { Zukunft und Wettbewerbsfähigkeit } \\
\text {-Arbeitsschwerpunkte des Befragten }\end{array}$ & $\begin{array}{l}\mathrm{x} \\
\mathrm{x} \\
\mathrm{x} \\
\mathrm{x}\end{array}$ & $\mathrm{x}$ \\
\hline Lohngestaltung im Betrieb & $\begin{array}{l}\text {-Bruttolöhne für Betriebsleitung, } \\
\text { Verwaltung, Tier- und } \\
\text { Pflanzenproduktion, Sonstiges } \\
\text {-Entlohnungssysteme auf Produktions- } \\
\text { und Führungsebene }\end{array}$ & $\mathrm{x}$ & $\mathrm{X}$ \\
\hline $\begin{array}{l}\text { Motivation und Arbeits- } \\
\text { verhalten der Mitarbeiter }\end{array}$ & $\begin{array}{l}\text {-Intrinsische Motivation } \\
\text {-Arbeitsverhalten }\end{array}$ & $\begin{array}{l}\mathrm{x} \\
\mathrm{x}\end{array}$ & $\mathrm{x}$ \\
\hline $\begin{array}{l}\text { Kommunikation der } \\
\text { Unternehmensziele }\end{array}$ & $\begin{array}{l}\text {-Zielklarheit } \\
\text {-Zielanzahl }\end{array}$ & $\begin{array}{l}\mathrm{x} \\
\mathrm{x}\end{array}$ & $\begin{array}{l}\mathrm{x} \\
\mathrm{x}\end{array}$ \\
\hline $\begin{array}{l}\text { Leistungsorientierte } \\
\text { Entlohnung }\end{array}$ & $\begin{array}{l}\text {-Erfahrungen mit leistungsorientierter } \\
\text { Entlohnung } \\
\text {-Leistungsindikatoren } \\
\text {-Anteil am Gesamtlohn }\end{array}$ & $\begin{array}{l}\mathrm{x} \\
\mathrm{x} \\
\mathrm{x}\end{array}$ & $\mathrm{x}$ \\
\hline $\begin{array}{l}\text { Materielle und immaterielle } \\
\text { Anreizsysteme }\end{array}$ & $\begin{array}{l}\text {-Vorhandenensein verschiedener } \\
\text { Anreizsysteme } \\
\text {-Wichtigkeit verschiedener } \\
\text { Anreizsysteme } \\
\text {-Statements zu verschiedenen } \\
\text { Anreizsystemen }\end{array}$ & $\mathrm{x}$ & $\mathrm{X}$ \\
\hline Arbeitszeitgestaltung & $\begin{array}{l}\text {-Statements zur } \\
\text { Arbeitszeitflexibilisierung }\end{array}$ & $\mathrm{x}$ & \\
\hline $\begin{array}{l}\text { Soziodemographische } \\
\text { Angaben }\end{array}$ & $\begin{array}{l}\text {-Bildungsgrad, Dauer der } \\
\text { Betriebszugehörigkeit bzw. -leitung, } \\
\text {-Alter, Geschlecht, Bundesland }\end{array}$ & $\mathrm{x}$ & $\mathrm{X}$ \\
\hline
\end{tabular}

Quelle: Eigene Darstellung

\footnotetext{
${ }^{18}$ Eine Likertskala dient der Einstellungsmessung. Hierfür wird dem Befragten eine Reihe von Aussagen (Statements) vorgelegt, denen er zustimmen oder ablehnen kann. Die Verwendung der Fünfer-Skala bietet stärkere Differenzierungsmöglichkeiten des Antwortverhaltens (DIEKMANN 2004: 182 f., FRIEDRICHS 1985: 175).
} 
Der nächste Teil des Betriebsleiterfragebogens befasst sich mit der Lohngestaltung im Betrieb. Hier werden die Arbeitnehmerbruttolöhne in den verschiedenen Hierarchieebenen und Arbeitsbereichen erfasst und die Lohngestaltung in den Betriebszweigen und auf Führungsebene abgefragt. Auch die Mitarbeiter wurden gefragt, unter welchem Entgeltsystem sie arbeiten.

Der darauf folgende Fragenkomplex, bestehend aus Statementbatterien, gilt der intrinsischen Motivation (aus Perspektive der Arbeitgeber und-nehmer) sowie dem Arbeitsverhalten der Mitarbeiter. Der sich anschließende Fragenkomplex zielt auf die Zielkommunikation im Unternehmen ab.

Die Erfahrungen der Betriebsleiter und Mitarbeiter mit leistungsorientierter Entlohnung, die von den Betriebsleitern eingesetzten Leistungsindikatoren und der Anteil des Leistungslohnes am Gesamtlohn sind weitere Variablen.

Um den Stellenwert und die Verwendung materieller und immaterieller Anreizsysteme bewerten zu können, sind mehrere Fragenblöcke diesem Thema gewidmet. Als eine Form immaterieller Anreizsysteme bietet sich möglicherweise eine flexible Arbeitszeitgestaltung an. Statements zu diesem Thema sind im Betriebsleiterfragebogen enthalten und wurden für ein Forschungsprojekt im Auftrag der Landwirtschaftlichen Rentenbank ausgewertet (vgl. DAVIER ET AL. 2006).

Im abschließenden Teil der Fragebögen wurden die soziodemographischen Angaben wie das Geschlecht, das Alter und die Dauer der Betriebszugehörigkeit bzw. der betriebsleitenden Tätigkeit erhoben.

\subsubsection{Datenerhebung und -auswertung der Betriebsleiterbefragung}

Die Betriebsleiterbefragung wurde in zwei Erhebungsphasen durchgeführt. Aus der ersten Erhebungsphase stammen 52 Datensätze mit dem Schwerpunkt in den alten Bundesländern. Diese wurden im Rahmen einer Studie für die Landwirtschaftliche Rentenbank zur Gestaltung von Anreizsystemen in landwirtschaftlichen Innovationsund Wachstumsprozessen ausgewertet.

In einer zweiten Erhebungsphase wurde die Befragung auf Ausbildungsbetriebe in Mecklenburg-Vorpommern und Thüringen ausgeweitet. Insgesamt wurden 501 Betriebe 
angeschrieben. 10 Tage nach dem Versand der Fragebögen, denen ein frankierter Rückumschlag beigelegt war, wurden die Betriebsleiter telefonisch an die Befragung erinnert. Die Rücklaufquote betrug insgesamt 38,75 \% (siehe Tabelle 12), d.h. 186 Betriebsleiter, die der Fragebogen erreichte, nahmen an der Befragung teil. Um einen Vergleich zwischen ökologisch und konventionell wirtschaftenden Großbetrieben durchführen zu können, wurde der gleiche Fragebogen außerdem durch die Bundesforschungsanstalt für Landwirtschaft an 89 ökologisch wirtschaftende Betriebe, die zum Praxis-Forschungs-Netz ökologischer Landbau gehören (RAHMANN ET AL. 2004), versandt. Hier von beantworteten 22 Betriebsleiter den Fragenbogen. Die angeschriebenen Landwirte wurden ebenfalls telefonisch an den Fragebogen erinnert.

TABELLE 12: RÜCKLAUFQUOTE DER BETRIEBSLEITERBEFRAGUNG, ZWEITE ERHEBUNGSPHASE

\begin{tabular}{|l|c|c|}
\hline Anzahl angeschriebene Betriebe & $\begin{array}{l}\text { Davon Adresse } \\
\text { unrichtig }\end{array}$ & Rücklaufquote in Prozent \\
\hline $\begin{array}{l}\text { 501 Ausbildungsbetriebe in Thüringen und } \\
\text { Mecklenburg-Vorpommern }\end{array}$ & 21 & $38,75 \%$ \\
\hline $\begin{array}{l}89 \text { ökologisch wirtschaftende Betriebe im } \\
\text { Bundesgebiet aus dem Praxis-Forschungsnetz } \\
\text { der FAL }\end{array}$ & 0 & $24,71 \%$ \\
\hline
\end{tabular}

Quelle: Eigene Darstellung

Die Auswahl der Betriebe zeigt, dass keine repräsentative Stichprobe vorliegt, sondern ein Convenience-Sample. Da der Fragebogen vor allem zum Test von Zusammenhangshypothesen dienen soll, ist nach DIEKMANN (2004: 369) in diesem Fall die Repräsentativität der Stichprobe entbehrlich.

Die Datenauswertung erfolgte mit dem Statistikprogramm SPSS für Windows in der Version 12.0. 


\subsection{Stichprobenstruktur}

\subsubsection{Stichprobenstruktur der Arbeitgeberbefragung}

Die Stichprobe umfasst 260 landwirtschaftliche Betriebsleiter. Von diesen haben rund $23 \%$ ihren Betriebssitz in den alten und $72 \%$ in den neuen Bundesländern. $5 \%$ der Betriebsleiter wollten keine Angabe zu ihrem Bundesland machen. Einige Betriebsleiter äußerten am Telefon die Sorge, dass ihr Betrieb über die Flächenausstattung direkt identifizierbar sei. Dies führte dazu, dass bestimmte Angaben nicht gemacht wurden. Hierzu zählten vor allem das Bundesland, der Anteil an Pacht- und Eigentumsfläche, die Anzahl der Arbeitskräfte und die bezahlten Stundenlöhne. 88,0 \% der Betriebe werden konventionell und $11,6 \%$ ökologisch ${ }^{19}$ bewirtschaftet. Im Vergleich zum bundesdeutschen Durchschnitt von 3,3 \% ökologisch wirtschaftenden Betrieben (BMVEL 2005: 41) ist dies ein überdurchschnittlicher Anteil ökologisch bewirtschafteter Betriebe. ${ }^{20}$ Die am häufigsten vertretenen Rechtsformen sind die Einzelunternehmen (28,1 \% aller Betriebe), GbR (22,3\%) und eG (21,5\%). Die restlichen Betriebe werden als $\mathrm{GmbH}$ (17,3\%), KG (6,5\%) oder sonstige Rechtsformen geführt. $^{21}$ Im Jahr 2003 waren in Deutschland 26,6\% aller ständig beschäftigten Fremdarbeitskräfte in Einzelunternehmen, 35,6 \% in juristischen Personen und 36,3\% in Personengesellschaften beschäftigt (BMVEL 2005: 53).

Die verschiedenen betriebswirtschaftlichen Ausrichtungen sind in der Stichprobe wie folgt vertreten: 30,5 \% der analysierten Betriebe sind Ackerbau-, 24,6 \% Verbund-, 17,3 \% Futterbau- und 7,7 \% Veredlungsbetriebe. 19,6 \% der Betriebe sind keiner der oben genannten betriebswirtschaftlichen Ausrichtungen eindeutig zuzuordnen. Ein Großteil dieser Kategorie entfällt auf Doppelnennungen. Am häufigsten wurden hier Acker- und Futterbau als wichtigste betriebswirtschaftliche Ausrichtungen genannt. In Deutschland waren im Jahr 2003 27,2 \% aller ständig beschäftigten familienfremden

\footnotetext{
${ }^{19}$ Teilnahme am EG-Kontrollverfahren nach den EG-Öko-Verordnungen 2092/91 bzw. 1804/99.

${ }^{20}$ Mit der Betriebsgröße in ha LF steigt auch der Anteil ökologisch wirtschaftender Betriebe an den Betrieben insgesamt. So haben die ökologisch wirtschaftenden Betriebe in der Größenklasse 200 ha und mehr LF einen Anteil von 6,0\% (ebenda).

21 Unter die Rubrik „sonstige Rechtsform“ fallen vor allem Betriebe, die Doppelnennungen von Rechtsformen vorgenommen haben. In diesem Fall wurden Teilbetriebe unter verschiedenen Rechtsformen geführt.
} 
Arbeitskräfte $^{22}$ in Ackerbau-, 27,7 \% in Futterbau-, 6,1 \% in Veredlungs- und 9,8 \% in Verbundbetrieben beschäftigt.

Alle Personen, die den Fragebogen beantworteten, befanden sich in Führungspositionen. In rund $95 \%$ der Fälle waren es Betriebsleiter bzw. Betriebsleiterinnen (8,5 \% der Fragebögen wurden von Frauen beantwortet). Der Bildungsgrad der befragten Personen ist sehr hoch. Nur $15 \%$ der kumulierten Häufigkeiten entfallen auf die Bildungsabschlüsse „landwirtschaftliche Lehre“, „staatlich geprüfter Wirtschafter“ und „Fachschule“. 68,8 \% der Befragten haben ein landwirtschaftliches Studium absolviert. Die Personen, die über keine landwirtschaftliche Ausbildung verfügen (3,1\%), gaben an, eine betriebswirtschaftliche Ausbildung bzw. ein Studium abgeschlossen zu haben. Der hohe Bildungsgrad bei den Betriebsleitern deckt sich mit Ergebnissen einer aktuellen Betriebsleiterbefragung zur Managementkapazität in Agrargenossenschaften und juristischen Personen Ostdeutschlands (TANNEBERGER UND JASTER 2006: 18). ${ }^{23}$ Interessant ist der höchst signifikante Unterschied zwischen dem Bildungsgrad der Betriebsleiter in den alten und neuen Bundesländern. Die Betriebsleiter der in der Stichprobe enthaltenen ostdeutschen Betriebe verfügen über einen höheren Bildungsgrad als die westdeutschen Betriebsleiter. RATHMANN ET AL. (2005: 21) konnten für Schleswig-Holstein zeigen, dass Betriebsleiter mit Fachhochschul- und Hochschulausbildung mit größerer Wahrscheinlichkeit Lohnarbeitskräfte einsetzen. Die Verfasser begründen dies mit dem Zusammenhang zwischen Qualifikation und den für die Leitung von Lohnarbeitsbetrieben notwendigen dispositiven Fähigkeiten.

Das durchschnittliche Alter der Betriebleiter liegt bei 48 Jahren, was auf erfahrene Betriebsleiter schließen lässt. Im Mittel bewirtschaften die Betriebsleiter ihre Betriebe seit 1991, dies untermauert die vorhergehende Schlussfolgerung.

Tabelle 13 zeigt die durchschnittliche Flächenausstattung und die Bestandesgrößen der analysierten Betriebe.

\footnotetext{
${ }^{22}$ Die betriebswirtschaftliche Ausrichtung „Gartenbau“ ist hier herausgerechnet.

${ }^{23} 80 \%$ der von TANNEBERGER UND JASTER (2006) befragten ostdeutschen Betriebsleiter verfügen über einen Hochschulabschluss.
} 
TABELLE 13: DURCHSCHNITTLICHE FLÄCHENAUSSTATTUNG UND BESTANDESGRÖßEN NACH BETRIEBSWIRTSCHAFTLICHER AUSRICHTUNG

\begin{tabular}{|c|c|c|c|c|}
\hline & $\begin{array}{c}\text { Alle } \\
\text { Betriebe }\end{array}$ & Ackerbaubetriebe & Futterbaubetriebe & Veredlungsbetriebe \\
\hline & \multicolumn{4}{|c|}{ Mittelwerte } \\
\hline Ackerfläche in ha & $\begin{array}{l}894,14 \\
\mathrm{n}=256\end{array}$ & $\begin{array}{c}781,11 \\
\mathrm{n}=79\end{array}$ & $\begin{array}{c}658,06 \\
\mathrm{n}=45\end{array}$ & $\begin{array}{c}403,27 \\
n=17\end{array}$ \\
\hline Grünland in ha & $\begin{array}{l}221,87 \\
\mathrm{n}=221\end{array}$ & $\begin{array}{c}92,95 \\
\mathrm{n}=60\end{array}$ & $\begin{array}{c}339,47 \\
\mathrm{n}=44\end{array}$ & $\begin{array}{c}333,48 \\
\mathrm{n}=6\end{array}$ \\
\hline Anzahl Milchkühe & $\begin{array}{c}340 \\
\mathrm{n}=143\end{array}$ & $\begin{array}{l}243 \\
n=9\end{array}$ & $\begin{array}{c}411 \\
n=37\end{array}$ & $\begin{array}{l}300 \\
n=1\end{array}$ \\
\hline Anzahl Mutterkühe & $\begin{array}{c}233 \\
\mathrm{n}=40\end{array}$ & $\begin{array}{c}76 \\
n=9\end{array}$ & $\begin{array}{c}304 \\
\mathrm{n}=10\end{array}$ & $\begin{array}{l}526 \\
\mathrm{n}=3\end{array}$ \\
\hline Mastschweineplätze & $\begin{array}{l}2.599 \\
\mathrm{n}=65\end{array}$ & $\begin{array}{c}2700 \\
n=3\end{array}$ & $\begin{array}{l}809 \\
\mathrm{n}=5\end{array}$ & $\begin{array}{l}6.637 \\
n=13\end{array}$ \\
\hline Zuchtsauenplätze & $\begin{array}{c}715 \\
\mathrm{n}=46\end{array}$ & $\begin{array}{c}5 \\
\mathrm{n}=1\end{array}$ & $\begin{array}{l}300 \\
n=1\end{array}$ & $\begin{array}{l}1.058 \\
\mathrm{n}=17\end{array}$ \\
\hline
\end{tabular}

Quelle: Eigene Erhebung, Betriebsleiterbefragung

In den befragten Ackerbaubetrieben werden durchschnittlich 781,11 ha Ackerfläche bewirtschaftet. Die durchschnittliche Ackerfläche aller in der Stichprobe enthaltenen Betriebe ist höher als die der spezialisierten Ackerbaubetriebe, da die LPGNachfolgeunternehmen über die meiste Ackerfläche verfügen, und häufig als Verbundbetriebe bewirtschaftet werden. Diese sind in der Tabelle nicht ausgewiesen. Dies erklärt auch die Differenz zwischen der Anzahl der Nennungen insgesamt und der Summe der Nennungen der Ackerbau-, Futterbau- und Veredlungsbetriebe. Die Veredlungsbetriebe verfügen im Mittel über 6.637 Mastschweineplätze bzw. 1.058 Plätze für Zuchtsauen. In den Futterbaubetrieben werden im Durchschnitt 411 Milchund 304 Mutterkühe gehalten. Durchschnittlich sind in den analysierten Betrieben 15,5 familienfremde Arbeitskräfte und 2,15 Familienarbeitskräfte beschäftigt. 
In der Abbildung 15 ist die Verteilung der Betriebe nach der Anzahl beschäftigter familienfremder Arbeitskräfte dargestellt. Danach werden in $45 \%$ der Betriebe 1-5 familienfremde Arbeitskräfte beschäftigt. Fast $46 \%$ der Betriebe beschäftigen sechs bis 20 familienfremde Arbeitskräfte. In über $9 \%$ der befragten Betriebe werden mehr als 40 Fremdarbeitskräfte beschäftigt.

\section{ABBILDUNG 15: FAMILIENFREMDE ARBEITSKRÄFTE IN DEN UNTERSUCHTEN BETRIEBEN}

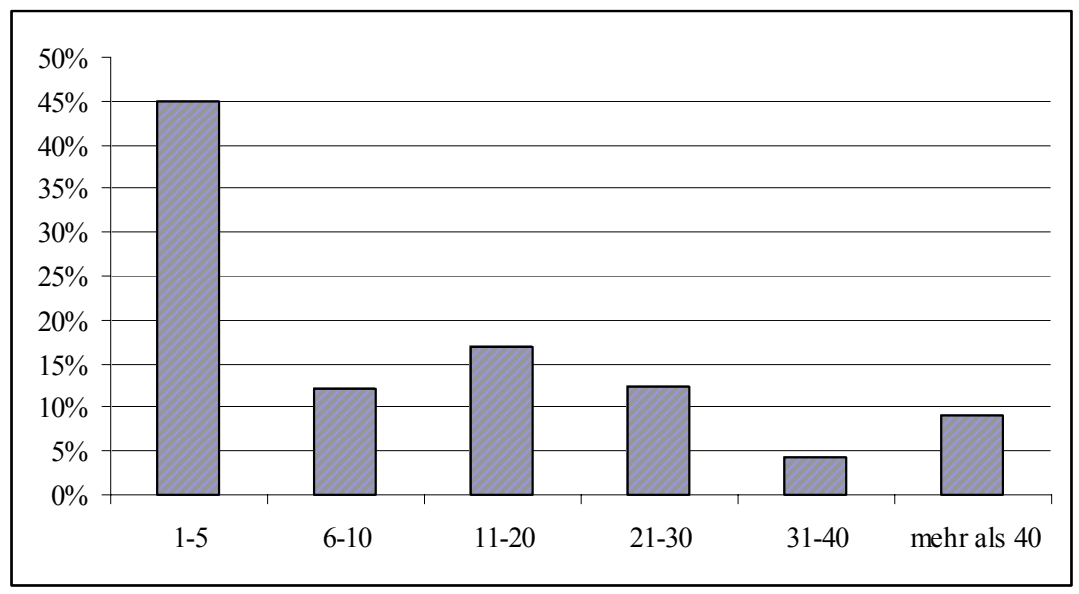

Quelle: Eigene Erhebung, Betriebsleiterbefragung

\section{Nutzung von Beratungsinstitutionen}

Die folgende Tabelle 14 zeigt, welche Institutionen die Beratung der analysierten Betriebe übernehmen. Die Agrarberatungsunternehmen könnten einen Einfluss auf die Wahl der Anreizsysteme haben, da z.B. bestimmte Entlohnungsschlüssel auch zum Beratungskonzept einer Organisation gehören können. Die Beratung auf dem Gebiet der Pflanzenproduktion übernehmen überwiegend die Offizialberater wie Landwirtschaftskammern, -ämter oder (dort wo vorhanden) die Pflanzenschutzämter. Private Beratungsbüros werden von über einem Drittel der Betriebsleiter für betriebswirtschaftliche Fragestellungen genutzt. Zwischen 18,8\% und $25 \%$ der Betriebsleiter kontaktieren Privatberater auch für produktionstechnische Fragestellungen. Die bedeutende Rolle der Ringberatung für produktionstechnische und betriebswirtschaftliche Fragestellungen (17,7-21,9 \%) ist zum Teil auf die regionalen Befragungsschwerpunkte zurückzuführen. Für produktionstechnische Probleme in der Tier- und Pflanzenproduktion werden aber auch häufig Firmenberater und andere Landwirte zu Rate gezogen. Von den sonstigen Beratungsträgern spielen die LMS (Landwirtschaftsberatung Mecklenburg-Vorpommern/Schleswig-Holstein) und der 
Genossenschaftsverband bei den befragten Betriebsleitern eine wichtige Rolle. Aber auch dies ist wiederum der regionalen Schwerpunktsetzung der Befragung geschuldet. Signifikante Unterschiede zwischen den Betrieben in den alten und neuen Bundesländern bestehen in der Bedeutung der Ringberatung. Betriebsleiter in den alten Bundesländern nutzen häufiger Ringberater für produktionstechnische und ökonomische Fragestellungen als die Betriebsleiter in den neuen Bundesländern.

TABELLE 14: NUTZUNG VON BERATUNGSTRÄGERN

\begin{tabular}{|l|c|c|c|c|}
\hline & \multicolumn{4}{|c|}{ Beratungsgebiet } \\
\hline \multicolumn{1}{|c|}{ Beratungsträger } & $\begin{array}{c}\text { Pflanzen- } \\
\text { produktion }\end{array}$ & Tierproduktion & $\begin{array}{c}\text { Betriebs- } \\
\text { wirtschaft }\end{array}$ & $\begin{array}{c}\text { Sonstige, z.B. } \\
\text { Steuer- } \\
\text { beratung, } \\
\text { Anträge, Biogas }\end{array}$ \\
\hline $\begin{array}{l}\text { Landwirtschafts- } \\
\text { kammer/-ämter/ } \\
\text { Pflanzenschutzamt }\end{array}$ & $54,2 \%$ & $18,1 \%$ & $16,9 \%$ & $5,4 \%$ \\
\hline $\begin{array}{l}\text { Private } \\
\text { Beratungsbüros }\end{array}$ & $24,2 \%$ & $18,8 \%$ & $33,5 \%$ & $6,2 \%$ \\
\hline Ringberatung & $19,6 \%$ & $21,9 \%$ & $17,7 \%$ & $2,3 \%$ \\
\hline Firmenberater & $43,1 \%$ & $33,5 \%$ & $6,5 \%$ & $3,1 \%$ \\
\hline $\begin{array}{l}\text { Andere Landwirte } \\
\text { Sonstige (u.a. } \\
\text { Genossenschafts- } \\
\text { verband, LMS, } \\
\text { Anbauverbände, } \\
\text { Bauernverband) }\end{array}$ & $24,6 \%$ & $19,2 \%$ & $8,1 \%$ & $4,6 \%$ \\
\hline
\end{tabular}

Quelle: Eigene Erhebung, Betriebsleiterbefragung, Mehrfachantworten sind möglich, N=252

\subsubsection{Stichprobenstruktur der Arbeitnehmerbefragung}

96,9 \% der Arbeitnehmer, die Angaben zum Bundesland gemacht haben, kommen aus Niedersachsen. Größtenteils sind die Mitarbeiter in Ackerbaubetrieben beschäftigt $(66,4 \%)$ und zu 93,4 \% übernehmen sie keine Betriebsleitungsfunktionen. Über 82,6 \% arbeiten nur im Ackerbau, 5,8 \% bezeichnen sich als Mitarbeiter in der Schweineproduktion und 3,3\% als Mitarbeiter in der Milchproduktion. Fast $80 \%$ der Mitarbeiter sind jedoch noch in anderen Betriebsbereichen im Einsatz. Besonders häufig 
sind die Mitarbeiter zusätzlich für die Maschinenreparaturen zuständig. Einige Mitarbeiter sind auch in der Saatzucht und der Saatgutaufbereitung tätig.

ABBILDUNG 16: HÖCHSTE BILDUNGSABSCHLÜSSE DER BEFRAGTEN MitARBEITER

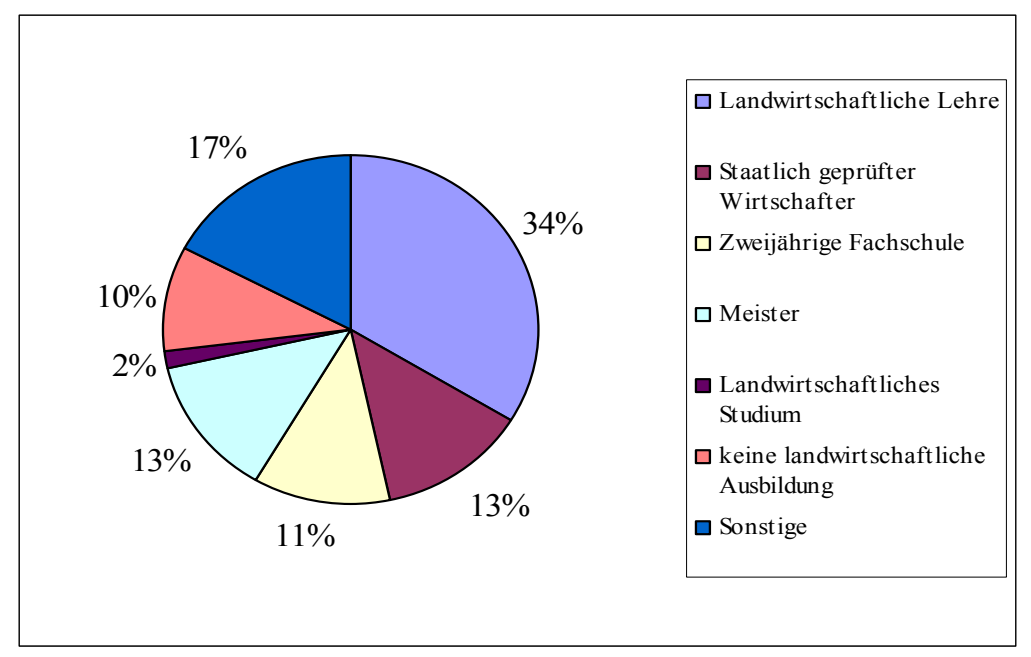

Quelle: Eigene Erhebung, Mitarbeiterbefragung

Abbildung 16 zeigt, wie sich die verschiedenen Bildungsabschlüsse auf die befragten Mitarbeiter verteilen. Fast $34 \%$ der Befragten verfügen über eine landwirtschaftliche Lehre als höchsten beruflichen Abschluss. $24 \%$ der befragten Mitarbeiter haben einen Fachschulabschluss. $13 \%$ haben erfolgreich die landwirtschaftliche Meisterschule besucht und $2 \%$ ein landwirtschaftliches Studium absolviert. $10 \%$ der Mitarbeiter haben keine landwirtschaftliche Ausbildung. Hierunter fallen ein studierter Betriebswirt, ein Kraftfahrzeugmechaniker oder andere Facharbeiter. Mitarbeiter, die die Antwortmöglichkeit „Sonstige“ (17 \%) gewählt haben, verfügen über eine landwirtschaftsnahe Ausbildung wie z.B. die Ausbildung zum Landmaschinenmechaniker, Gärtner oder landwirtschaftlich-technischen Assistenten (LTA).

Das Qualifikationsniveau macht deutlich, dass es sich bei den Befragten um gut ausgebildete Fachkräfte handelt. Es ist jedoch anzumerken, dass an den Winterschulungen in der Regel die Mitarbeiter teilnehmen, die Interesse an Weiterbildung haben, da sie einen Eigenanteil selbst entrichten müssen und - sofern sie nicht vom Betriebsleiter frei gestellt werden - Urlaub nehmen müssen. 


\subsection{Ergebnisse zu den Erfolgsbedingungen leistungsorientierter Entlohnung}

Im folgenden Kapitel werden die Ergebnisse der Arbeitgeber- und Arbeitnehmerbefragung $\mathrm{zu}$ den Erfolgsbedingungen leistungsorientierter Lohngestaltung dargestellt und der Bezug $\mathrm{zu}$ den theoretischen Hintergründen hergestellt. Zunächst werden die Ergebnisse der Faktorenanalyse zu den Erfolgsbedingungen leistungsorientierter Vergütung vorgestellt. Im Anschluss werden die einzelnen Faktoren, die den Erfolg eines leistungsorientierten Entgeltsystems bedingen, mit Hilfe von uni- und bivariaten Verfahren näher analysiert.

\subsubsection{Faktorenanalyse}

Bei der Mitarbeiterbefragung wurden in verschiedenen Fragen Items zur Tätigkeit, zur zielorientierten Steuerung und zur Lohngestaltung abgefragt. Die Variablen wurden mit Hilfe einer Faktorenanalyse zu vier Faktoren verdichtet. Die Faktorenanalyse dient zum einen der Dimensionsreduktion. Mit den daraus resultierenden zusammengefassten Faktoren können anschließend weitere Analysen vorgenommen werden. Zum zweiten vermittelt die Faktorenanalyse einen ersten Eindruck über den Zusammenhang zwischen verschiedenen Items.

Es wurde eine Hauptkomponentenanalyse verwendet, um eine Dimensionsreduktion vorzunehmen, die die Variablen so zusammenfasst, dass eine sie vereinende Bezeichnung gefunden werden kann. Außerdem wurde eine Varimax-Rotation durchgeführt. Die Varimax-Rotation stellt die Faktoren so zusammen, dass sie untereinander weitgehend unkorreliert sind. Das hat den Vorteil, dass die Faktoren für weitere Analysen weiterverwendet werden könnten, die eine Unkorreliertheit der Variablen voraussetzen (z.B. eine Clusteranalyse). Die durchgeführte Faktorenanalyse reduziert die Variablen auf vier Faktoren (vgl. 
Tabelle 15). 
TABELLE 15: ROTIERTE KOMPONENTENMATRIX ZU DEN ERFOLGSBEDINGUNGEN

\begin{tabular}{|c|c|c|c|c|}
\hline & \multicolumn{4}{|c|}{ Faktoren } \\
\hline & $\begin{array}{l}\text { Spaß an } \\
\text { der } \\
\text { Arbeit }\end{array}$ & $\begin{array}{l}\text { Erfolgreiche } \\
\text { Zielkommunikation }\end{array}$ & $\begin{array}{l}\text { Unzufriedenheit } \\
\text { mit der } \\
\text { Bezahlung }\end{array}$ & $\begin{array}{l}\text { Transparenz der } \\
\text { Lohngestaltung }\end{array}$ \\
\hline $\begin{array}{l}\text { "Die Arbeit in der } \\
\text { Landwirtschaft macht mir Spaß." }\end{array}$ & ,804 & & & \\
\hline $\begin{array}{l}\text { "Mein Arbeitseinsatz wird von } \\
\text { meinem Chef auch entsprechend } \\
\text { gewürdigt." }\end{array}$ & ,744 & & & \\
\hline $\begin{array}{l}\text { "Ich habe die Hilfsmittel und } \\
\text { Technik, die ich benötige, um } \\
\text { gute Arbeit zu leisten." }\end{array}$ & ,668 & & & \\
\hline $\begin{array}{l}\text { "Mein Chef bindet mich bei } \\
\text { betrieblichen Entscheidungen } \\
\text { ein." }\end{array}$ &, 530 & ,486 & & \\
\hline $\begin{array}{l}\text { "Mein Chef spricht regelmäßig } \\
\text { mit mir über die Ziele des } \\
\text { Unternehmens." }\end{array}$ & &, 869 & & \\
\hline $\begin{array}{l}\text { "Ich kann die Ziele des } \\
\text { Unternehmens klar benennen." } \\
\text { "Ich weiß, wie ich zur }\end{array}$ & &, 846 & & \\
\hline $\begin{array}{l}\text { Realisierung der } \\
\text { Unternehmensziele beitragen } \\
\text { kann." }\end{array}$ & & ,778 & & \\
\hline $\begin{array}{l}\text { "Ich fühle mich bei der } \\
\text { Bezahlung gegenüber meinen } \\
\text { Kollegen im Betrieb ungerecht } \\
\text { behandelt." }\end{array}$ & & &, 873 & \\
\hline $\begin{array}{l}\text { "In unserem Betrieb bekomme } \\
\text { ich für meine Tätigkeit weniger } \\
\text { Geld als in vergleichbaren } \\
\text { Betrieben." }\end{array}$ & & & ,797 & \\
\hline $\begin{array}{l}\text { "Auf Zuschläge und Prämien bin } \\
\text { ich finanziell angewiesen." }\end{array}$ & & & ,698 & \\
\hline $\begin{array}{l}\text { "Ich verstehe, wie Löhne und } \\
\text { Gehälter in unserem Betrieb } \\
\text { festgelegt werden." }\end{array}$ & & & & ,720 \\
\hline $\begin{array}{l}\text { "Zulagen, die ich für meine } \\
\text { Leistung erhalte, verbessern } \\
\text { meine Motivation." }\end{array}$ & & & & ,702 \\
\hline $\begin{array}{l}\text { "Mein Chef erläutert uns } \\
\text { ausführlich, wie Zulagen für } \\
\text { besondere Leistung zustande } \\
\text { kommen." }\end{array}$ & ,523 & & & ,620 \\
\hline Cronbachs Alpha & 0,695 & 0,775 & 0,731 & 0,687 \\
\hline
\end{tabular}

Quelle: Eigene Erhebung, Faktorladungen unter 0,35 sind in der Matrix unterdrückt, n=47

Das Maß der Stichprobeneignung wird mit dem Kaiser-Meyer-Olkin-Kriterium (KMO) angegeben. Der KMO-Wert beträgt in diesem Fall 0,728. Werte über 0,7 (BACKHAUS ET 
AL. 2000: 269) weisen auf eine ,ziemlich gute Eignung“ der Stichprobe hin. Die vier Faktoren erklären 70,25 \% der Varianz. Eine große Anzahl fehlender Werte bei den Statements, die sich auf die Bezahlung beziehen, führen $\mathrm{zu}$ einer deutlichen Verringerung des Stichprobenumfangs auf 47 Nennungen, mit denen die Faktorenanalyse durchgeführt wurde. BACKHAUS ET AL. (2003: 331) empfehlen, für die Faktorenanalyse nur Datensätze zu verwenden, deren Fallanzahl die Zahl der betrachteten Variablen um das mindestens dreifache übersteigt. Diesen Anforderungen genügt der vorliegende Datensatz.

Der erste Faktor kann als „Spaß an der Arbeit in der Landwirtschaft“ bezeichnet werden. Es zeigt sich ein starker positiver Zusammenhang zwischen der Freude an der Arbeit, der Würdigung des Arbeitseinsatzes durch den Chef, den Arbeitsmitteln, um gute Leistung auch erbringen $\mathrm{zu}$ können und der Einbindung in betriebliche Entscheidungen.

Im zweiten Faktor werden die Variablen zusammengefasst, die sich auf die Zielkommunikation im Betrieb beziehen. Dieser Faktor wird daher mit „Erfolgreiche Zielkommunikation“ tituliert. Eine erfolgreiche Kommunikation der Unternehmensziele ist eine wichtige Erfolgsbedingung für leistungsorientierte Entlohnung. Mitarbeitern, deren Chef regelmäßig mit ihnen über die Ziele im Unternehmen spricht, fällt es leichter, diese zu formulieren und zu ihrer Realisierung beizutragen.

Der dritte Faktor sammelt die Variablen, die mit der „Unzufriedenheit mit der Bezahlung“ im Zusammenhang stehen. Es besteht eine positive Wechselwirkung zwischen der Wahrnehmung von Fairnessdefiziten innerhalb und außerhalb der Betriebes und der persönlichen Bedeutung finanzieller Anreize.

Der letzte Faktor trägt den Namen „Transparenz der Lohngestaltung“. Analog zu den Erkenntnissen der Valenztheorien, steigt die Motivationswirkung von Leistungszulagen mit einer für die Mitarbeiter verständlichen Lohngestaltung.

Die Statements „Mein Chef bindet mich bei betrieblichen Entscheidungen ein“ und „Mein Chef erklärt uns ausführlich, wie Zulagen für besondere Leistungen zustande kommen" weisen hohe Ladungen auf zwei Faktoren auf. Sie wurden aus der Faktorenanalyse aufgrund ihres hohen Informationsgehalts nicht eliminiert. Es ist 
plausibel und theoretisch begründbar, dass die Einbindung in betriebliche Entscheidungen sowohl die Freude an der Arbeit fördert, als auch Ausdruck einer erfolgreichen Zielkommunikation ist. Die ausführlichere Erläuterung gewährter Leistungszulagen gegenüber den Mitarbeitern ist ein Zeichen für eine transparente Lohngestaltung, fördert aber gleichzeitig auch die Freude an der Arbeit. Der letztgenannte Zusammenhang ist aus der Perspektive der Lohngerechtigkeit zu begründen. Wenn den Mitarbeitern bewusst ist, wie Zulagen für besondere Leistung zustande kommen, sinkt nach Adams Equity Theory das Ungerechtigkeitsempfinden.

Durch die stark reduzierte Stichprobe stellt das Resultat der Faktorenanalyse nur ein erstes Zwischenergebnis dar, welches mit Hilfe von bivariaten Verfahren im Anschluss näher betrachtet werden soll. Offenbar gibt es nur einen geringen Zusammenhang zwischen der Freude an der Arbeit in der Landwirtschaft und der Zufriedenheit mit der Lohngestaltung und der Wertschätzung finanzieller Anreize.

\subsubsection{Motivation der Mitarbeiter in den untersuchten Betrieben aus Sicht der Betriebsleiter}

Die verschiedenen theoretischen Ansätze zur Gestaltung von Anreizsystemen zeigen, dass dem Mitarbeiter und seinen Präferenzen eine zentrale Rolle zukommt. In diesem Abschnitt ist zu klären, wie zufrieden die Arbeitgeber mit der Motivation ihrer Mitarbeiter sind und ob es Unterschiede zwischen den Betriebsleitern gibt. Die vorgestellten experimentellen Ergebnisse in Kapitel 3 und die Ausführungen zu den Erfolgsbedingungen leistungsorientierter Vergütung haben verdeutlicht, dass die intrinsische Motivation eine große Bedeutung hat, wenn es darum geht, die Wahrscheinlichkeit des Auftretens eines Verdrängungseffekt zu ermitteln. Um einen Einblick in die Motivation der Mitarbeiter in den untersuchten Betrieben zu erhalten, wurden die Betriebsleiter gebeten, eine Liste von Items mit Hilfe einer 5-stufigen Likertskala zu bewerten. Die Ergebnisse zeigt Abbildung 17. 


\section{ABBILDUNG 17: „MEINE MITARBEITER ERLEDIGEN IHRE ARBEIT GEWISSENHAFT UND ZU MEINER VOLLSTEN ZUFRIEDENHEIT“}

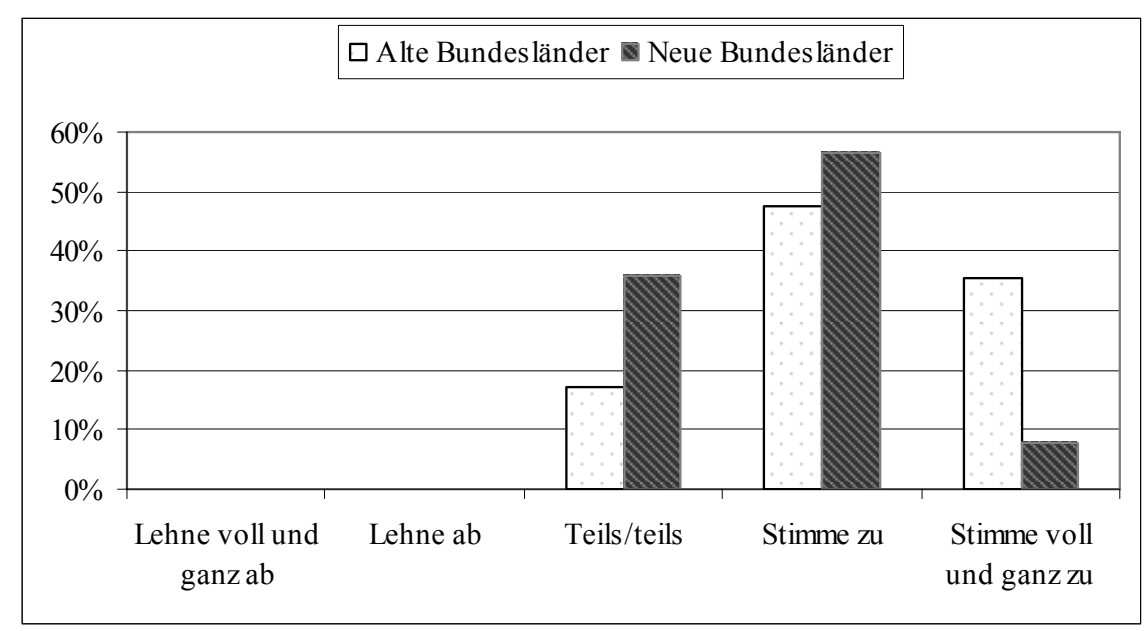

Quelle: Eigene Erhebung, fünfstufige Likertskala von -2 bis $+2, N=256$

Es wird deutlich, dass die Arbeitgeber im Großen und Ganzen mit der Arbeit ihrer Mitarbeiter sehr zufrieden sind. So entfallen $68 \%$ der gültigen Antworten auf die Kategorien „stimme zu“ und „stimme voll und ganz zu“. Keiner der Betriebsleiter, die das Statement beantwortet haben, lehnt dieses ab.

Ein Mittelwertvergleich zwischen den Betriebsleitern aus den neuen und alten Bundesländern ergibt einen höchst signifikanten Unterschied zwischen den beiden Gruppen. Die Betriebsleiter aus den alten Bundesländern bewerten das Statement durchschnittlich mit 1,19, d.h., sie sind besonders zufrieden mit der Arbeit ihrer Mitarbeiter. Die Betriebsleiter aus den neuen Bundesländern sind weniger zufrieden mit ihren Mitarbeitern als ihre Kollegen aus den alten Bundesländern. Ein möglicher Erklärungsgrund für das unterschiedliche Antwortverhalten könnte in der Betriebsgröße liegen, da durch das Statement alle Mitarbeiter bewertet werden müssen. Betriebe, die viele Mitarbeiter beschäftigen, müssen das Statement für alle Mitarbeiter bewerten. Da aber nicht immer alle Mitarbeiter gleich motiviert sind, wird die durchschnittliche Bewertung anders ausfallen als in einem Betrieb, in dem nur eine Lohnarbeitskraft beschäftigt ist. Unterschiede zwischen den Betriebsleitern könnten jedoch auch in unterschiedlichen Betriebsorganisationen, dem Lohnniveau oder Betriebsleitereigenschaften liegen. Dieser Frage wird im folgenden Abschnitt nachgegangen. 
Wie die Tabelle 16 zeigt, gibt es einen signifikanten bis hoch signifikanten Zusammenhang zwischen der Zufriedenheit der Betriebsleiter mit den Mitarbeitern und dem Lohnniveau in den untersuchten Betrieben. Der Zusammenhang ist in den Betrieben der alten Bundesländer ausgeprägter als in den neuen Bundesländern. Dies könnte sich auch aus den alternativen Beschäftigungsmöglichkeiten und dem Lohngefälle zwischen der Landwirtschaft und anderen Branchen erklären. Wenn es für die Mitarbeiter in der Region kaum andere Arbeitsplätze gibt, dann wird sich ein geringes Lohnniveau weniger stark auf die Motivation auswirken als in Regionen mit geringer Arbeitslosigkeit.

\section{TABELLE 16: KORRELATIONEN ZWISCHEN DER ZUFRIEDENHEIT MIT DEN MITARBEITERN UND VERSCHIEDENEN EINFLUSSGRÖßEN}

\begin{tabular}{|l|c|c|c|}
\hline & \multicolumn{2}{|c|}{$\begin{array}{c}\text { "Meine Mitarbeiter erledigen ihre Arbeit gewissenhaft und zu meiner } \\
\text { vollsten Zufriedenheit“ }\end{array}$} & NBL, $\mathrm{n}=184$ \\
\cline { 2 - 4 } & gesamt, $\mathrm{n}=256$ & ABL, $\mathrm{n}=59$ & $0,164^{*}$ \\
\hline $\begin{array}{l}\text { Bruttostundenlohn in } € / \mathrm{h} \text { ohne } \\
\begin{array}{l}\text { Zuschläge in der } \\
\text { Pflanzenproduktion }\end{array}\end{array}$ & $0,347^{* *}$ & $0,362^{*}$ & $-0,162^{*}$ \\
\hline $\begin{array}{l}\text { Anzahl der Lohnarbeitskräfte in } \\
\text { Personen }\end{array}$ & $-0,204^{* *}$ & $-0,070$ & \\
\hline
\end{tabular}

Quelle: Eigene Erhebung, Pearsons-Korrelationsindex ${ }^{24},{ }^{*} \mathrm{p}<0,05, * *<0,01$

Die Betriebsgröße hat einen geringeren Einfluss auf die Zufriedenheit der Betriebsleiter mit ihren Mitarbeiter als das Lohnniveau. Die Korrelation zwischen der Zufriedenheit mit den Mitarbeitern und der Anzahl der beschäftigten Mitarbeiter ist höher und signifikanter in den neuen als in den alten Bundesländern. Möglicherweise gibt es eine kritische Betriebsgröße bei der der Zusammenhang größer wird.

So wird in Abbildung 18 deutlich, dass die Leiter kleinerer Betriebe (gemessen an der Anzahl der Lohnarbeitskräfte) zufriedener mit ihren Mitarbeitern sind als die größerer Betriebe. Der Anteil der Betriebsleiter, die mit „teils/teils“ antworten, steigt mit zunehmender Anzahl der Lohnarbeitskräfte. Dieser Trend ist eindeutig im Bereich von

\footnotetext{
${ }^{24}$ Als Determinationsmaß wird der Pearson-Korrelationsindex (auch Produkt-Moment-Korrelationsindex) ausgewiesen, da es sich um zwei intervallskalierte Variablen handelt. Korrelationen zwischen ordinalen Variablen werden hingegen mit dem Spearman-Korrelationsindix bestimmt (BÜHNER 2004: 247 ff).
} 
1-20 Arbeitskräften. Auch geht mit der Anzahl der Lohnarbeitskräfte der Anteil der Betriebsleiter, die dem Statement voll zustimmen, zurück.

ABBILDUNG 18: ANZAHL DER LOHNARBEITSKRÄFTE UND ZUFRIEDENHEIT MIT DEN MITARBEITERN

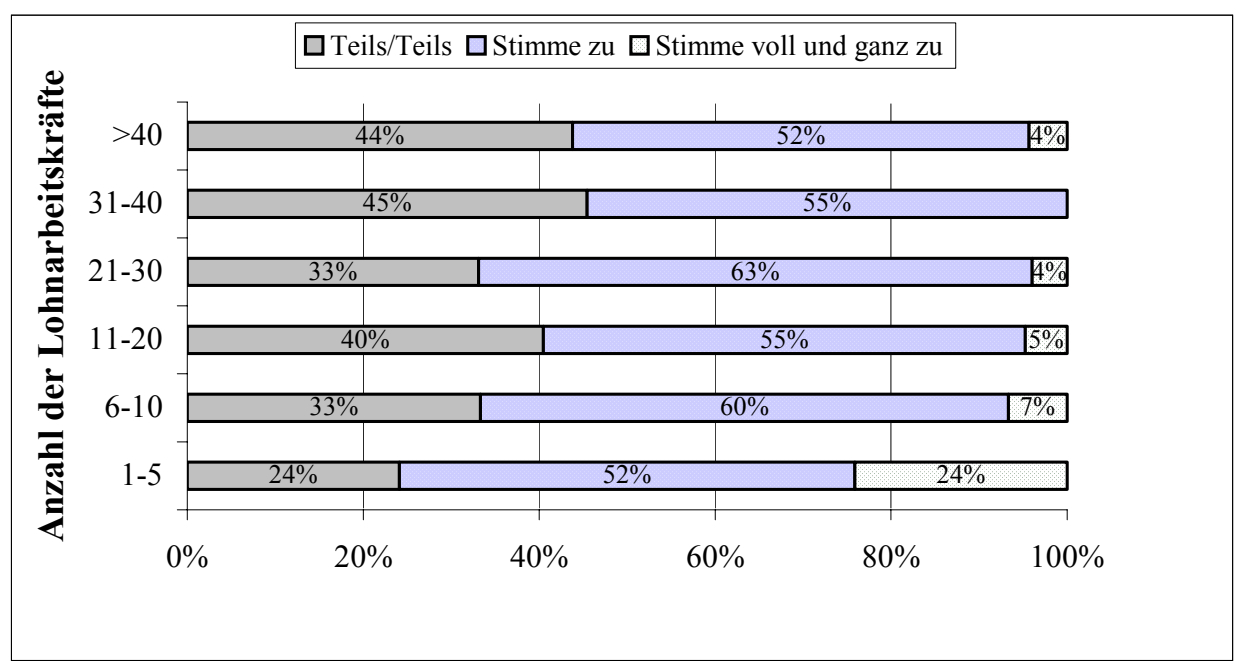

Quelle: Eigene Erhebung

Ein Ziel der Befragung ist es, einen Eindruck über die intrinsische Motivation und das Arbeitsverhalten der Mitarbeiter zu erhalten. Die Abbildung 19 verdeutlicht die Einschätzung der Betriebsleiter zur intrinsischen Motivation und zum Arbeitsverhalten ihrer Mitarbeiter. 
ABBILDUNG 19: POLARITÄTENPROFIL ZUR MOTIVATION UND DEM ARBEITSVERHALTEN

\begin{tabular}{|c|c|c|c|c|c|c|c|}
\hline & \multicolumn{2}{|c|}{ gesamt, $n=256$} & \multicolumn{2}{|c|}{$\begin{array}{l}\text { trifft } \\
\text { überhaupt } \\
\text { nicht zu }\end{array}$} & \multicolumn{3}{|c|}{$\begin{array}{l}\text { trifft voll und } \\
\text { ganz zu }\end{array}$} \\
\hline & $\sigma$ & $\mu$ & -2 & -1 & 0 & 1 & 2 \\
\hline $\begin{array}{l}\text { Meinen Mitarbeitern geht es nicht } \\
\text { nur ums Geld, ihnen macht die } \\
\text { Arbeit Spaß. }\end{array}$ & 0,71 & 0,694 & & & & & \\
\hline $\begin{array}{l}\text { Meine Mitarbeiter hängen an der } \\
\text { Arbeit in der Landwirtschaft. }\end{array}$ & 0,85 & 0,674 & & & & & \\
\hline $\begin{array}{c}\text { Meine Mitarbeiter sind bereit auch } \\
\text { mal länger zu arbeiten. }\end{array}$ & 1,50 & 0,577 & & & & & \\
\hline $\begin{array}{l}\text { Meine Mitarbeiter erledigen ihre } \\
\text { Aufgaben selbständig. }\end{array}$ & 0,78 & 0,708 & & & & & \\
\hline $\begin{array}{l}\text { Meine Mitarbeiter erledigen ihre } \\
\text { Arbeit gewissenhaft und zu meiner } \\
\text { vollsten Zufriedenheit. }\end{array}$ & 0,83 & 0,656 & & & & & \\
\hline
\end{tabular}

Quelle: Eigene Erhebung, neue Bundesländer: ........gestrichelte Linie, __ alte Bundesländer: dunkle Linie

Insgesamt überwiegt ein sehr positiver Eindruck über die intrinsische Motivation und das Arbeitsverhalten der Mitarbeiter. Sie führen Arbeiten selbständig aus und sind bereit länger zu arbeiten.

Die Arbeitgeber sind überwiegend der Meinung, dass es ihren Mitarbeitern nicht nur ums Geld geht, sondern dass ihnen die Arbeit Spaß macht. Außerdem haben sie den Eindruck, dass ihre Mitarbeiter an der Arbeit in der Landwirtschaft hängen. Die Betriebsleiter in den neuen Bundesländern unterscheiden sich im ersten Punkt jedoch signifikant von denen in den alten Bundesländern.

Es wird deutlich, dass die Betriebsleiter sich insgesamt sehr einig über die Bereitschaft ihrer Mitarbeiter zur Ableistung von Überstunden sind. Die Bereitschaft zu längeren Arbeitszeiten scheint für die Mitarbeiter selbstverständlich $\mathrm{zu}$ sein. Signifikante Unterschiede gibt es hier nicht zwischen den Betriebsleitern der alten und neuen Bundesländer sondern einzig zwischen den unterschiedlichen Wirtschaftsweisen (in Abbildung 19 nicht dargestellt). Die Betriebsleiter der ökologisch wirtschaftenden Betriebe stimmen diesem Statement weniger zu. Dies könnte auf einen unterschiedlichen Arbeitsethos oder auf die ohnehin hohe Arbeitsbelastung in ökologisch wirtschaftenden Betrieben hinweisen. Dies müsste in weitergehenden Analysen, die eine breitere Datenbasis umfassen, untersucht werden. 
Neben den Unterschieden zwischen den Betriebsleitern der alten und neuen Bundesländer in der Zufriedenheit mit den Mitarbeitern, wird deutlich, dass sich die Betriebsleiter auch in ihrer Einschätzung zur Arbeitseinstellung ihrer Mitarbeiter unterscheiden. Die Bereitschaft zu längeren Arbeitszeiten und die selbständige Arbeitsweise sind in den neuen Bundesländern weniger ausgeprägt als in den alten Bundesländern. In den Ergebnisworkshops wurde von Mitarbeiterseite angemerkt, dass manche Agrargenossenschaften Wochenendarbeit vermeiden, da sie aufgrund des überhöhten Personalbestandes Überstunden vermeiden wollen. In diesem Fall würde die Bereitschaft der Mitarbeiter zur Mehrarbeit auch durch den Betriebsleiter beeinflusst werden.

Aus den Einschätzungen der Betriebsleiter zur Motivation ihrer Mitarbeiter lassen sich für die leistungsorientierte Lohngestaltung die folgenden Schlussfolgerungen ziehen. Die Zufriedenheit der Betriebsleiter mit der Arbeitsleistung und Motivation ihrer Mitarbeiter ist zunächst eine positive Nachricht für die Landwirtschaft. Auch lässt sich daraus zunächst kein Handlungsbedarf ableiten. Die Betriebsleiter sind bereits zufrieden mit ihren Mitarbeitern. Das hohe Maß an intrinsischer Motivation würde gegen eine leistungsorientierte Entlohnung sprechen und das Augenmerk eher auf intrinsische Anreize wie die Anerkennung der Arbeit lenken. Denn die Gefahr, dass ein Verdrängungseffekt auftritt, ist dann besonders hoch, wenn die Mitarbeiter besonders intrinsisch motiviert sind.

\subsubsection{Intrinsische Motivation der befragten Arbeitnehmer}

Die positive Einschätzung der Betriebsleiter über die intrinsische Motivation der Mitarbeiter deckt sich mit den Ergebnissen der Arbeitnehmerbefragung. Die befragten Mitarbeiter bewerten das Statement „Die Arbeit in der Landwirtschaft macht mir Spaß“ auf einer Likert-Skala von -2 bis +2 durchschnittlich mit 1,71 .

Chi²-Tests zwischen dem Statement „Spaß in der Arbeit“ und der Beschäftigungsdauer (in Jahren) im Betrieb, der Anzahl der Beschäftigten und dem Bildungsgrad ergaben keine signifikanten Unterschiede zwischen den Mitarbeitern. 
Die Ausführungen zum Verdrängungseffekt zeigten, dass die Wechselwirkung zwischen extrinsischen Anreizen und intrinsischer Motivation positiv, neutral oder negativ sein können (vgl. Kapitel 3.3.2).

In der folgenden Tabelle 17 sind Korrelationsindizes zwischen dem Statement „Die Arbeit in der Landwirtschaft macht mir Spaß“ und Statements zur Motivationswirkung von Zulagen, Gerechtigkeitsempfinden gegenüber der Referenzgruppen und der Wertschätzung finanzieller Anreize dargestellt. Es wird deutlich, dass die intrinsische Motivation und eine positive Motivationswirkung von Zulagen unkorreliert sind. Der Zusammenhang zwischen dem Spaß an der Arbeit in der Landwirtschaft und der Wertschätzung finanzieller Anreize ist negativ, wenn auch nicht besonders hoch. Eine stärkere negative und signifikante Beziehung gibt es zwischen der intrinsischen Motivation und einem wahrgenommenen Ungerechtigkeitsempfinden in Bezug auf die Höhe der Bezahlung im Vergleich zu den Mitarbeitern im Betrieb und in vergleichbaren Betrieben. Dies bestätigt die Equity-Theorie von Adams, nach der ungerechte Behandlung im Vergleich zu Referenzpersonen zu einem Motivationsrückgang führt.

TABELLE 17: INTRINSISCHE MOTIVATION UND EXTRINSISCHE ANREIZE

\begin{tabular}{|l|c|c|c|c|}
\hline & $\begin{array}{c}\text { Positive } \\
\text { Motivationswirkung } \\
\text { von Zulagen }\end{array}$ & $\begin{array}{c}\text { Wahrgenommene } \\
\text { ungerechte } \\
\text { Bezahlung } \\
\text { gegenüber der } \\
\text { Referenzgruppe im } \\
\text { Betrieb }\end{array}$ & $\begin{array}{c}\text { Unterdurchschnittliche } \\
\text { Bezahlung im } \\
\text { Verhältnis zu } \\
\text { vergleichbaren } \\
\text { Betrieben }\end{array}$ & $\begin{array}{c}\text { Wertschätzung } \\
\text { finanzieller } \\
\text { Anreize }\end{array}$ \\
\hline $\begin{array}{l}\text { Intrinsische } \\
\text { Motivation }\end{array}$ & 0,036 & $-0,258^{*}$ & $-0,225^{*}$ & $-0,196$ \\
\hline
\end{tabular}

Quelle: Eigene Erhebung, Mitarbeiterbefragung, Pearsons-Korrelationsindex, ${ }^{*} \mathrm{p}<0,05$

Betrachtet man anschließend die Beziehung zwischen der Freude an der Arbeit in der Landwirtschaft und verschiedenen immateriellen Anreizen (Tabelle 18), so zeigt sich, dass die intrinsische Motivation der Mitarbeiter mit den immateriellen Anreizen „Anerkennung der Arbeit“, „Einbindung in betriebliche Entscheidungen“ und „Erreichbarkeit der vom Betriebsleiter gesetzten Unternehmensziele“ positiv korreliert ist. Die stärkste Beziehung besteht offensichtlich zwischen der Freude an der Arbeit und einer angemessenen Anerkennung der Arbeitsleistung. 
TABELLE 18: INTRINSISCHE MOTIVATION UND IMMATERIELLE ANREIZE

\begin{tabular}{|l|l|l|l|}
\hline & $\begin{array}{l}\text { Würdigung der } \\
\text { Arbeitsleistung durch den } \\
\text { Chef }\end{array}$ & $\begin{array}{l}\text { Einbindung bei } \\
\text { betrieblichen } \\
\text { Entscheidungen }\end{array}$ & $\begin{array}{l}\text { Erreichbarkeit der durch } \\
\text { den Betriebsleiter } \\
\text { gesetzten } \\
\text { Unternehmensziele }\end{array}$ \\
\hline $\begin{array}{l}\text { Intrinsische } \\
\text { Motivation }\end{array}$ & $0,460^{* *}$ & $0,346^{* *}$ & $0,363^{* *}$ \\
\hline
\end{tabular}

Quelle: Eigene Erhebung, Mitarbeiterbefragung, Pearsons-Korrelationsindex, ${ }^{*} \mathrm{p}<0,05,{ }^{*} \mathrm{p}<0,01$

Als Schlussfolgerung aus den Ausführungen zur intrinsischen Motivation der Mitarbeiter lässt sich ableiten, dass es bei den befragten Mitarbeiter offensichtlich ein hohes Maß an intrinsischer Motivation gibt. Die Ergebnisse der Befragung legen nahe, dass die Wechselwirkung von intrinsischer Motivation und extrinsischen Anreizen tendenziell eher negativ ist. Hingegen zeigen die Analysen, dass immaterielle Anreize zu einer stärkeren positiven Motivationswirkung als die extrinsischen Anreize führen. Dies würde die Wahrscheinlichkeit des Auftretens eines Verdrängungseffekts erhöhen. Die Ergebnisse bestätigen außerdem die Herzberg'sche Zweifaktorentheorie (vgl. Kapitel 3.2.1), nach der die Arbeitszufriedenheit im Wesentlichen vom Arbeitsinhalt und Anerkennung der Arbeitsleistung abhängt. Die Diskussion mit den befragten Mitarbeitern ergab, dass Feedback eine wichtige Ursache für Motivation und fehlende Rückkopplung ein bedeutender Grund für Demotivation ist.

\subsubsection{Zielorientierte Steuerung}

In Kapitel 3.4 wurde gezeigt, dass die zielorientierte Steuerung ein wichtiges Erfolgskriterium für leistungsorientierte Entlohnung ist. Die Festlegung klarer Ziele verbessert den Erfolg leistungsorientierter Entlohnung, da hierdurch die Mitarbeiter in die Lage versetzt werden, der Wahrscheinlichkeit, Ziele zu erreichen, eine realistische subjektive Einschätzung zu verleihen.

Abbildung 20 zeigt die Einschätzung der Mitarbeiter und Betriebsleiter zur zielorientierten Steuerung aus den beiden durchgeführten Befragungen. 
ABBILDUNG 20: EINSCHÄTZUNG DER BETRIEBSLEITER UND MITARBEITER ZUR ZIELKLARHEIT

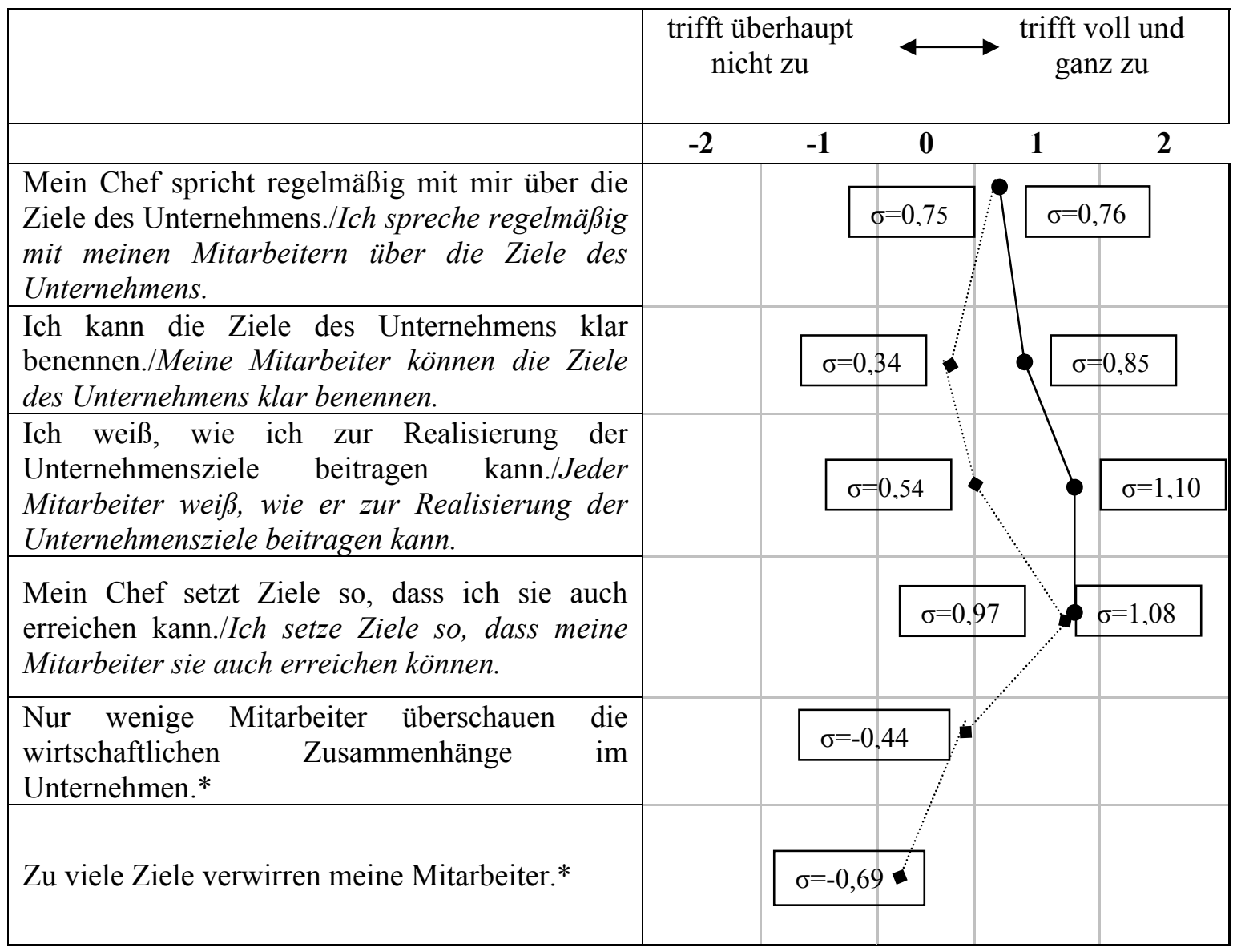

Quelle: Eigene Darstellung, Betriebleiter gestrichelt mit Rauten, Mitarbeiter, schwarze Linie, *Item wurde in der Mitarbeiterbefragung nicht abgefragt.

Es wird deutlich, dass die Mitarbeiter die Statements zur zielorientierten Steuerung im Mittel günstiger einschätzen als die Betriebsleiter. Übereinstimmungen finden sich nur in einem Statement, das sich auf die Kommunikation der Unternehmensziele bezieht.

Es gibt verschiedene Erklärungsmöglichkeiten für die Abweichungen im Antwortverhalten von Arbeitgebern und -nehmern:

1. Die Unterschiede können den explorativen Charakter der Untersuchung widerspiegeln, da Arbeitgeber und Arbeitnehmer nicht aus den gleichen Unternehmen stammen.

Ein Mittelwertvergleich zwischen den Arbeitgebern aus den alten und neuen Bundesländern, den verschiedenen betriebswirtschaftlichen Ausrichtungen, Rechtsformen und Betriebsgrößen zeigt aber den gleichen Trend wie das 
Polaritätenprofil. Die Einschätzung der Betriebsleiter ist immer ungünstiger als die der Mitarbeiter. T-Tests ergeben signifikante Unterschiede auf einen Niveau von $p<0,1$ zwischen den Betriebsleitern in den alten und neuen Bundesländern in den drei ersten Statements. Die Betriebsleiter aus den neuen Bundesländern kommunizieren nach ihrer Aussage regelmäßiger mit ihren Mitarbeitern über die Ziele des Unternehmens, schätzen aber die Mitarbeiter schlechter ein, was ihre Fähigkeiten zur Formulierung der Unternehmensziele betrifft.

2. Die Divergenz zwischen Arbeitgebern und -nehmern kann Ausdruck von unterschiedlicher Selbst- und Fremdwahrnehmung sein. Folgt man diesem Erklärungsansatz, so könnten die Antworten der Mitarbeiter als Selbstüberschätzung interpretiert werden oder die Einschätzung der Betriebsleiter als Unterschätzung ihrer Beschäftigten. PORTER UND LAWLER bezeichnen dies als unterschiedliche Rollenwahrnehmung, die den Grad der Leistungserfüllung bestimmt. Wenn die Einschätzung von Arbeitnehmern und Arbeitgebern hinsichtlich der Unternehmensziele stark voneinander abweichen, dann kann sich dies auf die Leistung der Mitarbeiter negativ auswirken, auch wenn sich diese sehr bemühen. Ihr Bemühen würde sich dann auf die falschen Ziele richten, die der Betriebsleiter nicht als leistungsrelevant einschätzt.

Eine Überprüfung dieser Interpretation ist aufgrund des Studiendesigns nicht möglich, da Betriebsleiter und Mitarbeiter nicht dem gleichen Betrieb entstammen.

3. Die Formulierung der Statements ist nicht eindeutig genug. Sie werden von Betriebsleitern und Mitarbeitern unterschiedlich interpretiert.

Diesem Erklärungsansatz folgend, könnten die Betriebsleiter die Ziele als langfristig und strategisch ansehen, während die Mitarbeiter die Unternehmensziele als kurzfristig und operativ interpretiert haben. Aufgrund der schriftlichen Befragungssituation ist dieser Sachverhalt ex post nicht mehr zu klären. Es empfiehlt sich aber in weiteren Studien, die Statements noch eindeutiger zu formulieren.

Betrachtet man die Beantwortung der Statements zur zielorientierten Steuerung von Arbeitnehmern und -gebern einmal isoliert voneinander, so ergibt sich für die leistungsorientierte Lohngestaltung in den Betrieben die folgende Schlussfolgerung: Die 
günstige Einschätzung der Mitarbeiter zu diesen Statements führt dazu, dass eine wichtige Erfolgsbedingung für Leistungslöhne in den Betrieben, in denen die befragten Arbeitnehmer arbeiten, erfüllt ist. Es ist jedoch auch an dieser Stelle darauf hinzuweisen, dass die befragten Mitarbeiter nicht den Durchschnitt der landwirtschaftlichen Lohnarbeitskräfte repräsentieren, sondern in Bezug auf Qualifikation und Motivation als überdurchschnittlich angesehen werden müssen. Ihnen sind die Unternehmensziele vermutlich klarer als weniger interessierten und engagierten Mitarbeitern.

Folgt man den Einschätzungen der Betriebsleiter zur zielorientierten Steuerung in den Betrieben, dann legen die Ergebnisse einen Verbesserungsbedarf nahe. Da die zielorientierte Steuerung in den Betriebsleiterbetrieben noch nicht optimal ist, kann diese Rahmenbedingung für ein leistungsabhängiges Vergütungssystem noch verbessert werden. In diesem Zusammenhang ist ein weiteres interessantes Ergebnis, dass die Betriebsleiter nicht der Meinung sind, zu viele Ziele würden ihre Mitarbeiter verwirren. Dies zeigt entweder ein positives Bild der Betriebsleiter über ihre Mitarbeiter oder ein mangelndes Bewusstsein für die Notwendigkeit der Formulierung weniger Ziele.

\subsubsection{Zum Stellenwert extrinsischer Anreize}

Eine weitere Erfolgsbedingung für leistungsorientierte Entlohnung ist die Wertschätzung finanzieller Anreize durch die Mitarbeiter, die sich aus dem Modell von PORTER UND LAWLER (vgl. Kapitel 3.2.4) ableitet.

Zunächst soll die relative Bedeutung der Lohngestaltung im Vergleich zu anderen Anreizsystemen dargestellt werden. Die Mitarbeiter wurden gebeten, eine Auswahl von zehn Anreizsystemen nach ihrer Wichtigkeit zu sortieren. ${ }^{25}$ Die Arbeitgeber wurden gefragt, ob die in der Auswahl enthaltenen immateriellen Anreizsysteme im Betrieb Anwendung finden.

Abbildung 21 zeigt die fünf wichtigsten Anreizsysteme aus Mitarbeitersicht. Für 54,2 \% der Mitarbeiter ist ein sicherer Arbeitsplatz der wichtigste Anreiz, eine hohe Arbeitsleistung $\mathrm{zu}$ erbringen, gefolgt vom guten Betriebsklima (42,5\%). Die

${ }^{25}$ Neben den in Abbildung 21 dargestellten fünf wichtigsten Anreizsystemen standen zur Auswahl: Betriebsfeste, Fort- und Weiterbildungsangebote, zusätzliche Sozialleistungen und individuelle Arbeitszeitgestaltung und Sonstiges. 
Lohngestaltung (Höhe und Art der Bezahlung) ist nur für $34 \%$ der Mitarbeiter das wichtigste Anreizsystem und rangiert an dritter Stelle. An vierter Stelle kommt der Arbeitsinhalt. Das fünftwichtigste Anreizsystem ist die Arbeit mit modernen Maschinen $(22,9 \%)$.

\section{ABBILDUNG 21: DIE FÜNF WICHTIGSTEN ANREIZSYSTEME AUS MitARBEITERSICHT}

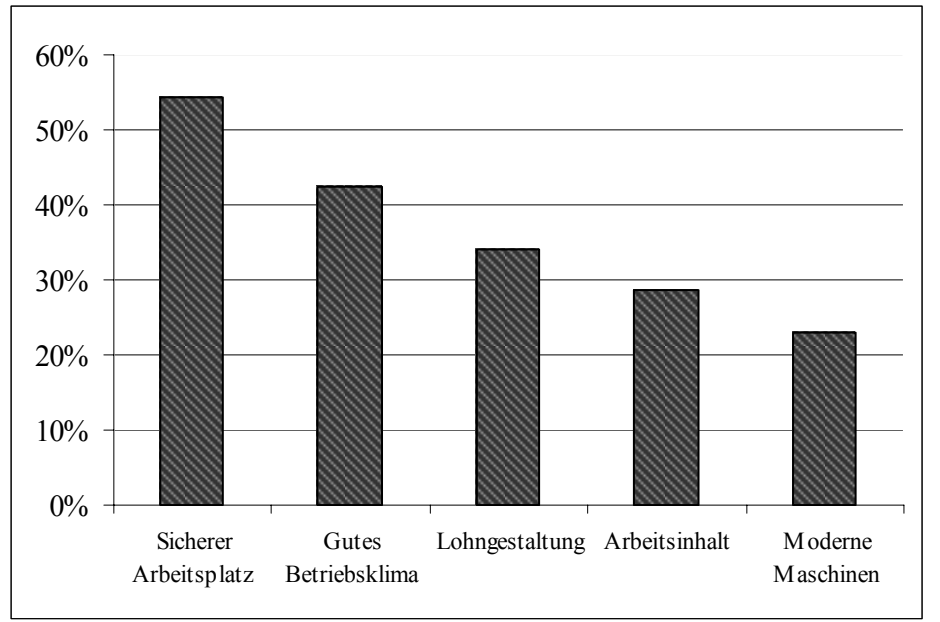

Quelle: Eigene Erhebung, Mitarbeiterbefragung

In der folgenden Tabelle 19 ist ausgewiesen, wie häufig die Betriebsleiter die von den Mitarbeitern in eine Rangfolge sortierten Anreizsysteme anbieten.

TABELLE 19: ANREIZSYSTEME IN DEN UNTERSUCHTEN BETRIEBEN

\begin{tabular}{|l|c|}
\hline Gutes Betriebsklima & $84,0 \%$ \\
\hline Sicherer Arbeitsplatz & $78,6 \%$ \\
\hline Moderne Maschinen & $71,2 \%$ \\
\hline Betriebsfeste & $71,2 \%$ \\
\hline $\begin{array}{l}\text { Individuelle Arbeitszeitgestaltung (z.B. Arbeitszeitausgleich, Abbummeln von } \\
\text { Überstunden, Teilzeit) }\end{array}$ & $65,4 \%$ \\
\hline Zusätzliche Sozialleistungen (z.B. Altersvorsorge) & $54,3 \%$ \\
\hline Gratifikationen (Geschenke, Zuwendungen, etc.) & $52,9 \%$ \\
\hline Fort- und Weiterbildungsangebote & $52,1 \%$ \\
\hline
\end{tabular}

Quelle: Eigene Erhebung, Betriebsleiterbefragung, Mehrfachantworten sind möglich

Das wichtigste Anreizsystem aus Mitarbeitersicht, den sicheren Arbeitsplatz, bieten $78,6 \%$ der befragten Betriebsleiter. $84 \%$ der Betriebsleiter glauben, dass sie über ein gutes Betriebsklima verfügen. $16 \%$ teilen diese Meinung nicht. $71,2 \%$ der 
Betriebsleiter haben moderne Maschinen und richten Betriebsfeste für ihre Mitarbeiter aus. Individuelle Arbeitszeitgestaltung ist in 64,5 \% der Betriebe möglich. In 54,3 \% der Betriebe erhalten die Mitarbeiter zusätzliche Sozialleistungen zum Beispiel zur Altersvorsorge. Über 50 \% der Betriebsleiter bieten ihren Mitarbeitern Gratifikationen und die Möglichkeit zur Fort- und Weiterbildung. Ein Mitarbeiter berichtete im Ergebnisworkshop, dass der Kindergartenplatz von seinem Betrieb bezahlt wird. Als sonstige Anreizsysteme wurden Deputate, Familienanschluss, Unterstützung im privaten Umfeld, Einbeziehung der Familie, Mitbewirtschaftung mitarbeitereigener Flächen, Unterstützung mit Maschinen, Einbeziehung beim Maschinenkauf oder bei Investitionsentscheidungen und ein monatliches Gespräch über aktuelle Probleme und Ziele im Betrieb genannt. Die Auswertungen zeigen, dass die überwiegende Zahl der Betriebsleiter die von den Mitarbeitern als wichtig betrachteten Anreizsysteme bieten, so dass die Bedürfnisse der Mitarbeiter mit dem Angebot der Betriebsleiter weitgehend übereinstimmen.

Einen weiteren Hinweis auf die Wertschätzung finanzieller Anreize liefert die folgende Abbildung 22.

\section{ABBILDUNG 22: WERTSCHÄTZUNG FINANZIELLER ANREIZE}

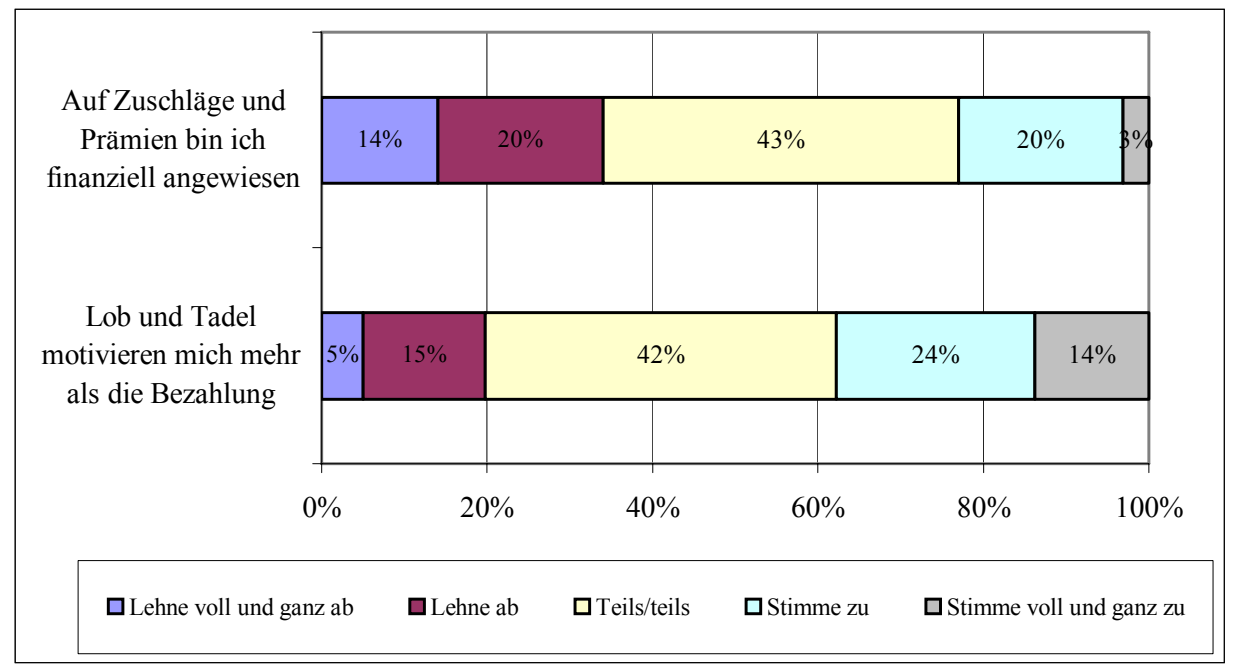

Quelle: Eigene Erhebung, Mitarbeiterbefragung

Es wird deutlich, dass die Mitarbeiter auf Zuschläge und Prämien nur zum Teil angewiesen sind. Durchschnittlich beantworten die befragten Mitarbeiter das Statement „Auf Zuschläge und Prämien bin ich finanziell angewiesen“ mit -0,23. Mehr Mitarbeiter 
lehnen das Statement ab als ihm zustimmen. Auch stehen die Mitarbeiter dem Statement „Lob und Tadel motivieren mich mehr als die Bezahlung“ ambivalent gegenüber und bewerten es im Mittel mit 0,26.

Die Beantwortung der Statements erlaubt folglich keine eindeutige Aussage hinsichtlich der Wertschätzung extrinsischer Anreize. Die Auswertungen erlauben jedoch den Schluss, dass Motivationsinstrumente wie die Sicherheit des Arbeitsplatzes, ein gutes Betriebsklima und andere immaterielle Anreize von größerer Bedeutung sind als die Entlohnung und vom überwiegenden Teil der Betriebsleiter geboten werden.

Damit leistungsorientierte Entlohnungssysteme auch verhaltensbeeinflussend wirken, sollte der Anteil am Gesamtlohn 15-30 \% ausmachen (GRIESEL 2004: 6). Von den Betriebsleitern, die leistungsorientierte Entlohnungssysteme anwenden, machten 68 Personen eine Angabe zur prozentualen Bedeutung des Vergütungssystems. Durchschnittlich hat der Leistungslohn einen Anteil von 15,7 \% am Gesamtlohn, der Median beträgt 12,5\% bei einem Minimalwert von $1 \%$ und einem Maximalwert von $70 \%$. D.h., der Leistungslohn dürfte im Durchschnitt von den Mitarbeitern wahrgenommen werden, da er einen merkbaren Anteil am Gesamtlohn einnimmt. 


\subsection{Lohngestaltung in den untersuchten Betrieben}

\subsubsection{Entlohnungssysteme bei den Mitarbeitern in der Produktion}

Für die unterschiedlichen Produktionsrichtungen wurden die in den Betrieben angewendeten Entlohnungssysteme erhoben (vgl. Tabelle 20).

TABelle 20: ENTLOHNUngSsysteme BeI DEN MitARBEITERn IN DER PFLANZEN- UND TIERPRODUKTION

\begin{tabular}{|l|c|c|c|}
\hline & $\begin{array}{c}\text { Ackerbau } \\
n=241\end{array}$ & $\begin{array}{c}\text { Milchproduktion } \\
n=139\end{array}$ & $\begin{array}{c}\text { Schweineproduktion } \\
n=70\end{array}$ \\
\hline Fester Stundenlohn/Gehalt & $94,2 \%$ & $92,1 \%$ & $91,4 \%$ \\
\hline $\begin{array}{l}\text { Höhergruppierung in } \\
\text { Lohnstufen in Abhängigkeit } \\
\text { von der Leistung }\end{array}$ & $26,6 \%$ & $27,3 \%$ & $20,0 \%$ \\
\hline Überstundenzuschläge & $35,3 \%$ & $23,7 \%$ & $27,1 \%$ \\
\hline $\begin{array}{l}\text { Zuschläge für Sonn- und } \\
\text { Feiertagsarbeit }\end{array}$ & $56,4 \%$ & $59,7 \%$ & $52,9 \%$ \\
\hline $\begin{array}{l}\text { Zulagen für } \\
\text { Arbeitserschwernis }\end{array}$ & $17,9 \%$ & $16,5 \%$ & nicht abgefragt \\
\hline Leistungszulagen & $15,4 \%$ & $36,0 \%$ & $7,2 \%$ \\
\hline Prämien & $16,2 \%$ & $23,2 \%$ & $24,3 \%$ \\
\hline Erfolgsbeteiligungen & $17,4 \%$ & & $26,1 \%$ \\
\hline
\end{tabular}

Quelle: Eigene Erhebung, Betriebsleiterbefragung, Mehrfachantworten möglich

Überwiegend werden die Mitarbeiter im Ackerbau sowie in der Milch- und Schweineproduktion durch einen festen Stundenlohn bzw. ein Gehalt entlohnt. Im Ackerbau ist der Anteil des Zeitlohns höher als in der Milch- und Schweineproduktion. In Kapitel 4 wurde deutlich, dass Zeitlohn und fixes Gehalt besonders vorteilhaft sind, wenn die Leistung der Mitarbeiter nicht zuverlässig messbar ist und es schwierig ist, objektive Leistungsindikatoren zu finden. In den Ausführungen zu den Besonderheiten der landwirtschaftlichen Produktion wurde gezeigt, dass der Einfluss stochastischer Größen auf den Produktionsprozess im Ackerbau besonders stark ist. So ist es folgerichtig, dass hier feste Entgeltsysteme überwiegen. Aber auch die Milch- und Schweineproduktion basieren auf biologischen Prozessen, die nicht vollständig automatisierbar sind. Außerdem hat die Leistung in den erhobenen 
Produktionsrichtungen vielfältige Dimensionen mit starken Interdependenzen. Gerade bei Tätigkeiten, die einer hohen Sorgfalt bedürfen, haben feste Entlohnungssysteme ihre Stärke. In der heutigen Landwirtschaft erfordern die meisten Arbeitsgänge in der Tierund Pflanzenproduktion eine sorgfältige Ausführung der Arbeit und sind zumeist verbunden mit Überwachungsaufgaben. Einfache manuelle Tätigkeiten, wie das Ausmisten oder Hoffegen, die nur einer begrenzten Arbeitsqualität bedürfen, sind in der Landwirtschaft inzwischen eher die Ausnahme.

Die große Bedeutung inputbasierter Entlohnungssysteme führt jedoch nicht dazu, dass in den untersuchten Betrieben leistungsorientierte Entlohnungssysteme keine Rolle spielen.

Leistungsorientierte Entlohnungssysteme wie Leistungszulagen, Prämien und reine Leistungslöhne (ohne Grundlohn bzw. Gehalt) spielen in den untersuchten Betrieben in der Tierproduktion eine bedeutendere Rolle als in der Pflanzenproduktion. Bei den Entgeltsystemen mit Leistungsbezug dominieren aber die Mischformen zwischen einem in der Regel anforderungsabhängigen Grundlohn und einer auf subjektiven Kriterien beruhenden Leistungszulage oder einem Prämienlohn, der auf objektiven Leistungsindikatoren beruht.

Leistungszulagen werden von 15,4 \% der befragten Betriebsleiter im Ackerbau gewährt. In der Milchproduktion sind es 16,5 \% der Betriebsleiter, die Leistungszulagen einsetzen. In einigen Betrieben erhalten die Arbeitnehmer Zulagen für wenige Krankheitstage oder Unfälle. In der Schweineproduktion spielt die Leistungszulage eine untergeordnete Rolle, sie wird nur von 7,2 \% der Betriebsleiter eingesetzt.

Der Anteil der Betriebsleiter, der Prämienlöhne einsetzt, unterscheidet sich stark zwischen den verschiedenen Produktionsrichtungen. So werden in $16,2 \%$ der analysierten Ackerbaubetrieben Prämienlöhne verwendet. 36 \% der Betriebsleiter in der Milchproduktion und 24,3\% der Betriebsleiter in der Schweineproduktion wenden Prämienlöhne an. Auf die verwendeten Leistungsindikatoren wird in Kapitel 5.4.3 näher eingegangen.

Die in den untersuchten Milchvieh und Schweine haltenden Betrieben angewendeten reinen Leistungslöhne sind Teamvergütungen. Hier werden die Mitarbeiter nach dem 
Endprodukt vergütet. In der Milchviehproduktion erhalten die Mitarbeiter in einem Betrieb monatlich 5 Cent pro Liter ermolkener Milch, die auf alle Mitarbeiter in der Milchproduktion nach geleisteten Arbeitsstunden verteilt wird.

Hinsichtlich der Verwendung von Leistungslöhnen lässt sich aus dem vorhandenen Datenmaterial nur eine tendenzielle Schlussfolgerung ziehen. Offensichtlich werden in der Milchproduktion in dieser Stichprobe am häufigsten leistungsorientierte Entlohnungssysteme eingesetzt. Hervorzuheben ist die bedeutende Rolle von Prämienlohnsystemen in der Milchproduktion. Den befragten Betriebsleitern gelingt es offenbar, trotz der bestehenden innerbetrieblichen Wechselwirkungen zwischen Milchproduktion und Futterbau geeignete Leistungsindikatoren für Prämienlöhne zu finden.

Auch in der Schweineproduktion ist dies noch eher als im Ackerbau möglich, wie die Ergebnisse zeigen. Wie auch in der Milchproduktion tendieren die Betriebsleiter dieses Betriebszweigs in der Verwendung von Leistungslöhnen stärker zu Prämienlöhnen als $\mathrm{zu}$ Leistungszulagen. Aus motivationstheoretischer Sicht ist die Verwendung von objektiven Leistungskriterien, auf denen Prämienlohnsysteme basieren, zu befürworten.

Das Verhältnis von Leistungszulagen zu Prämienlöhnen ist im Ackerbau im Vergleich zur Milch- und Schweineproduktion eher ausgewogen. Dies verdeutlicht die Schwierigkeit, im Ackerbau objektive, kardinal messbare Leistungskriterien zu finden.

Eine interessante Lösung, um die individuelle Arbeitsleistung in die Berechnung von Zeitlohn und Gehalt einzubeziehen, haben 20-30 \% der befragten Betriebsleiter in den verschiedenen Produktionsrichtungen gewählt: Wie in Kapitel 4 dargestellt wurde, beruht die Eingruppierung in Lohngruppen in den gängigen Arbeitsbewertungsverfahren auf der Messung der mit den verschiedenen Tätigkeiten verbundenen Anforderungen und notwendigen Qualifikation. Die Einstufung der Mitarbeiter in Lohngruppen nach ihrer Leistung entspricht dieser üblichen Vorgehensweise nicht. Die Leistungsbeurteilung in den analysierten Betrieben zur Einstufung in die verschiedenen Lohngruppen beruht eher auf subjektiven als objektiven Kriterien. So wird zum Beispiel die Sorgfalt bei der Arbeit oder die Arbeitsgeschwindigkeit als Bemessungsgrundlage herangezogen. Dies kann als eine Form der relativen Leistungsentlohnung in landwirtschaftlichen Betrieben interpretiert 
werden. Die Beförderungsmöglichkeiten sind in der Landwirtschaft nur sehr begrenzt. Entwicklungsmöglichkeiten, die sich im Lohn ausdrücken, können folglich nur über einen höheren Stundenlohn oder, dort wo sie anwendbar ist, durch eine hohe Leistungsvergütung zum Ausdruck kommen. Die Hochstufung auf ein höheres Stundenlohnniveau durch gute Leistung entspricht dem Charakter eines Turniers, denn nicht jeder Mitarbeiter wird jährlich ein höheres Lohnniveau erreichen. Zur Einordnung der subjektiven Leistung des Mitarbeiters wird der Betriebsleiter auch die Leistung der anderen Kollegen als Vergleichsgrundlage heranziehen.

Erfolgsorientierte Entlohnung ist zwar keine leistungsorientierte Entgeltform, sondern lediglich eine variable Entlohnungsform, da der Betriebserfolg nicht unmittelbar von der individuellen Arbeitsleistung der Mitarbeiter abhängt. Die Beteiligung der Mitarbeiter am Erfolg oder Misserfolg des Unternehmens wird von 17,4 \% (im Ackerbau) bis 26,3\% der Betriebsleiter (in der Schweineproduktion) vorgenommen. Wie auch bei den leistungsorientierten Entlohnungssystemen spielen erfolgsabhängige Entgelte bei den Mitarbeitern im Pflanzenbau eine geringere Rolle als in der Tierproduktion.

Die überdurchschnittliche Länge der Arbeitszeiten in der Landwirtschaft und der notwendige Arbeitseinsatz auch an Sonn- und Feiertagen spiegeln sich ebenfalls in Tabelle 20 wieder. In 40-50 \% der Fälle erhalten die Mitarbeiter Überstundenzuschläge. Außerdem werden in 53-60 \% der Betriebe, unabhängig von der Produktionsrichtung, Zuschläge für Sonn- und Feiertagsarbeit gewährt.

Zulagen für Arbeitserschwernis - z.B. für das Ausbringen von Pflanzenschutzmitteln sind in einigen Bundesländern (z.B. Schleswig-Holstein) tarifvertraglich vereinbart.

Die Bedeutung der verschiedenen Entlohnungsformen in den unterschiedlichen Betriebszweigen bestätigen im wesentlichen die Ergebnisse älterer Studien zu Entlohnungssystemen in der Landwirtschaft (DOLUSCHITZ ET AL. 1996, BECKMANN 2000, WÜLLMERS 1995).

Chi²-Tests zeigen signifikante Zusammenhänge zwischen der Rechtsform und der Anwendung leistungsorientierter Entlohnungsformen. In Tabelle 21 werden aus diesem Grunde exemplarisch die Häufigkeiten dieser Vergütungsformen in Einzelunternehmen 
und in der Rechtsform der eG aufgeführt, um die Hypothese $2 \mathrm{zu}$ prüfen, in der angenommen wurde, dass in LPG-Nachfolgeunternehmen leistungsorientierte Entlohnung eine größere Rolle als in Einzelunternehmen spielen.

TABELLE 21: RECHTSFORMUNTERSCHIEDE IN DER ENTLOHNUNGSFORM

\begin{tabular}{|l|c|c|}
\hline & Einzelunternehmen & $e G$ \\
\hline Leistungszulagen im Ackerbau & $\begin{array}{c}18,2 \% \\
\mathrm{n}=66\end{array}$ & $\begin{array}{c}16,4 \% \\
\mathrm{n}=55\end{array}$ \\
\hline Leistungszulagen in der & $\begin{array}{c}29,4 \% \\
\mathrm{n}=17\end{array}$ & $\begin{array}{c}36 \% \\
\mathrm{n}=50\end{array}$ \\
\hline Milchproduktion & $\begin{array}{c}13,6 \% \\
\mathrm{n}=66\end{array}$ & $\begin{array}{c}21,8 \% \\
\mathrm{n}=55\end{array}$ \\
\hline Prämienlöhne im Ackerbau & $\begin{array}{c}5,9 \% \\
\mathrm{n}=17\end{array}$ & $\begin{array}{c}24,0 \% \\
\mathrm{n}=50\end{array}$ \\
\hline Prämienlöhne in der & & \\
\hline
\end{tabular}

Quelle: Eigene Erhebung, Betriebsleiterbefragung

Es zeigt sich, dass im Ackerbau in den Einzelunternehmen etwas häufiger Leistungszulagen eingesetzt werden als in den eingetragenen Genossenschaften. Prämienlöhne werden im Ackerbau hingegen deutlich häufiger von den eingetragenen Genossenschaften verwendet.

In der Milchproduktion spielen hingegen Leistungszulagen und Prämien in den Einzelunternehmen eine geringere Rolle als in den Genossenschaften. Der Unterschied zwischen den Rechtsformen ist im Vergleich zum Ackerbau jedoch nicht signifikant.

\subsubsection{Leistungsindikatoren in den analysierten Betrieben}

Die befragten Betriebsleiter verwenden verschiedene Leistungsindikatoren zur Bemessung von Leistungszulagen oder Prämien. Die folgenden Tabellen (vgl. Tabelle 22 bis Tabelle 24) zeigen, welche Indikatoren im Ackerbau, in der Milchproduktion und in der Schweineproduktion eingesetzt werden. Eine ausführliche Diskussion der Leistungskennzahlen und Handlungsempfehlungen für die Praxis finden sich jedoch in Kapitel 6. Die Prozentangaben beziehen sich immer auf die Betriebsleiter, die leistungsorientierte Entlohnungssysteme im jeweiligen Betriebszweig anwenden. 
LANDWIRTSCHAFT

TABELLE 22: LEISTUNGSINDIKATOREN IM ACKERBAU

\begin{tabular}{|l|c|}
\hline Arbeitsleistung (z.B. Druschleistung, gepflügte Fläche/h) & $48,4 \%$ \\
\hline Sorgfältiger Umgang mit Maschinen & $35,5 \%$ \\
\hline Reparaturkosten & $29 \%$ \\
\hline Deckungsbeitrag des Betriebszweigs & $25,8 \%$ \\
\hline Maschinenkosten & $22,6 \%$ \\
\hline Hektarerträge & $21 \%$ \\
\hline Sparsamer Einsatz von Pflanzenschutzmitteln & $1,6 \%$ \\
\hline
\end{tabular}

Quelle: Eigene Erhebung, Betriebsleiterbefragung, Mehrfachantworten möglich, N=62

48,4 \% der Betriebsleiter machen die leistungsorientierte Entlohnung an der Quantität der Arbeitsleistung, wie z.B. der Druschleistung in $\mathrm{t} / \mathrm{h}$ oder der gepflügten Fläche/h fest.

Eine besondere Rolle spielen in den untersuchten Betrieben Ersparnisprämien. So orientieren sich bei der Bemessung von Prämien 22,6 \% der Betriebsleiter an den Maschinen- und $29 \%$ an den Reparaturkosten. D.h., wenn ein gewisses Budget an Maschinenunterhaltungskosten pro Jahr unterschritten wird, erhalten die Mitarbeiter einen Teil davon.

Auch der sparsame Einsatz von Diesel, gemessen in Litern, wird in einigen Betrieben zusätzlich belohnt. Ausführungen $\mathrm{zu}$ den Anwendungsvoraussetzungen dieses Leistungsindikators finden sich in Kapitel 6.2.1. In einigen Betrieben erhalten die Mitarbeiter ein so genanntes Werkzeuggeld. Beispielsweise erhalten die Mitarbeiter $40 €$ Werkzeuggeld bei vorhandener Grundausstattung. Wenn nichts verloren geht, dürfen sie das Werkzeuggeld behalten. Die Gewährung von Werkzeuggeld wird von einem mündlich befragten Betriebsleiter einer Agrargenossenschaft sehr kritisch gesehen. Seiner Meinung nach ist diese Art der Ersparnisprämie noch zu kompliziert zu berechnen und würde den Betriebserfolg nicht wesentlich beeinflussen. Es sei wichtig, auf die Stellen vor und nicht nach dem Komma zu achten (Betriebsleiter A). Dies verdeutlicht das nachfolgende Zitat:

„Wenn mein Angestellter im Milchviehstall sehr gute Leistung bringt, ist es mir egal, ob er dafür zwei Schippen und drei Besen verbraucht hat.“ (Betriebsleiter A). 
Zur Vermeidung von durch Nachlässigkeit entstandene Schäden erhalten die Mitarbeiter in einem Betrieb eine Prämie von $1.000 € / J a h r$. Arbeiten sie nachlässig, so wird der daraus resultierende Schaden von dieser Summe abgezogen.

Qualitätsprämien werden in den untersuchten Betrieben zum Beispiel für die Silagequalität oder die Anerkennung von Vermehrungskartoffeln gewährt. Das erste Beispiel verdeutlicht die Problematik der innerbetrieblichen Wechselbeziehungen zwischen verschiedenen Betriebszweigen. Durch die Berücksichtigung der Silagequalität wird im oben genannten Beispiel das Verantwortungsbewusstsein der Mitarbeiter in der Grundfutterbergung und -bereitung und das Bewusstsein für die Konsequenzen einer guten Futterqualität in der Milchviehhaltung gestärkt.

In einem Teil der analysierten Betriebe werden bei der Entlohnung von Führungskräften im Ackerbau der sparsame Einsatz von Pflanzenschutzmitteln und die Einhaltung agrotechnischer Termine ${ }^{26}$ zusätzlich honoriert.

Die befragten Betriebsleiter beteiligen ihre Mitarbeiter darüber hinaus am Erfolg des Betriebszweiges. Sei es durch Prämien, die sich am Hektar-, am Reinertrag oder Deckungsbeitrag des Betriebszweigs orientieren, oder durch zusätzliche Beiträge zur Altersversorgung, die vom Betriebsergebnis abhängen.

26 Die Einhaltung ,agrotechnischer“ Termine wurde von einem Betriebsleiter als weiterer Leistungsindikator genannt. Unter dem Begriff ist die termingerechte Durchführung pflanzenbaulicher Maßnahmen zu verstehen. 
LANDWIRTSCHAFT

TABELLE 23: LEISTUNGSKRITERIEN IN DER MILCHPRODUKTION

\begin{tabular}{|l|c|}
\hline Milchqualität & $77,6 \%$ \\
\hline Milchleistung & $65,7 \%$ \\
\hline Reproduktionsrate & $20,9 \%$ \\
\hline Zellzahlen & $47 \%$ \\
\hline Tierarztkosten & $14,9 \%$ \\
\hline Kälberverluste & $43,3 \%$ \\
\hline Zwischenkalbezeit & $11,9 \%$ \\
\hline Deckungsbeitrag des Betriebszweigs & $9 \%$ \\
\hline Grundfutterleistung & $1,5 \%$ \\
\hline
\end{tabular}

Quelle: Eigene Erhebung, Betriebsleiterbefragung, Mehrfachantworten möglich, N=67

In der Milchproduktion ist die Milchqualität das häufigste Leistungskriterium (77,6 \%), gefolgt von der Milchleistung (65,7 \%) und den Zellzahlen (47 \%).

Neben Milchqualität und -leistung ist die Höhe der Kälberverluste ein häufiges Kriterium zur Bemessung von Prämien, sie wurde von 43,3 \% der Betriebsleiter genannt.

Außerdem werden Fruchtbarkeitsparameter wie die Reproduktionsrate (20,9 \% der Betriebsleiter), die Zwischenkalbezeit (11,9 \%), der Besamungsindex, die Trächtigkeitsrate und die Brunstbeobachtung zur Bemessung von Prämien herangezogen. Die letzten drei Parameter nannten die Betriebsleiter unter der Antwortmöglichkeit „Sonstiges“

Als Leistungsindikator für die Tiergesundheit, der in die Bezahlung einfließt, wird die Höhe der Tierarztkosten von 14,9 \% der Betriebsleiter genannt.

Die Grundfutterleistung der Tiere spielt als Bemessungsgrundlage für Leistungslöhne keine bedeutende Rolle (1,5\%). Unter der Antwortkategorie „Sonstiges“ nannten die Betriebsleiter die Qualität der Fütterung gemessen am Harnstoffwert, Ordnung und Sauberkeit und saubere Siloanschnittsflächen. 
In einem Betrieb wird die Höhe der Prämie genauer spezifiziert: Die Mitarbeiter erhalten zum Grundlohn einen Zuschlag von einem Euro, der sich in Abhängigkeit von Totgeburten, Milchmenge, -qualität und Tierarztkosten ergibt.

$9 \%$ der Betriebsleiter nehmen den Deckungsbeitrag des Betriebszweiges als Grundlage einer zusätzlichen Entlohnung und einige Mitarbeiter werden auch am Betriebsergebnis beteiligt.

Wie auch im Ackerbau werden für die Mitarbeiter der Milchproduktion zum Teil Beiträge zur Altersversorgung in Abhängigkeit vom Betriebsergebnis geleistet.

TABELLE 24: LEISTUNGSKRITERIEN IN DER SCHWEINEPRODUKTION

\begin{tabular}{|l|c|}
\hline Aufgezogene Ferkel/Sau/Jahr & $83,3 \%$ \\
\hline Ferkelverluste & $30,4 \%$ \\
\hline Futterkosten & $13,0 \%$ \\
\hline Tägliche Zunahmen & $8,7 \%$ \\
\hline Magerfleischanteil & $4,3 \%$ \\
\hline
\end{tabular}

Quelle: Eigene Erhebung, Betriebsleiterbefragung, Mehrfachantworten möglich, N=24

In der Sauenhaltung ist die Anzahl der aufgezogenen Ferkel pro Sau und Jahr das häufigste Kriterium zur Bemessung von Leistungslöhnen. Dies nannten 83,3 \% der Betriebsleiter. Als weiterer Bezugszeitraum für die Anzahl der abgesetzten Ferkel wird noch der Monat oder das Quartal verwendet. Kürzere Zeiträume als Bemessungsgrundlage haben den Vorteil, dass Handlung und Ergebnis für die Mitarbeiter besser abzusehen sind, so dass Verhaltensveränderungen schneller vorgenommen werden können. Ein möglicher Nachteil ist, dass Schwankungen in der Leistung der Tiere sich zwar im Mittel des Jahres nivellieren, sie aber im betreffenden Monat bzw. dem Quartal direkt auf den Mitarbeiter wirken, auch wenn sie nicht durch eine schlechte Arbeitsleistung begründet sein müssen. Ein weiterer Parameter ist die Anzahl der verkauften Ferkel pro Sau und Jahr. Mit 30,4 \% der Nennungen folgen die Ferkelverluste als wichtiges Leistungskriterium. Als Kriterium für die Güte des Herdenmanagements werden die Umrauscher pro Sau und Quartal herangezogen. 
In der Mastschweinehaltung sind außerdem die Futterkosten von Bedeutung (13\% der befragten Schweinehalter) sowie der Magerfleischanteil und die Zunahmen pro Tag nach ausgestallten Abteilungen.

Die Anzahl der verkauften Schweine und die Erfüllung von QS sind ein weiteres Leistungskriterium in den untersuchten Betrieben.

Wie auch im Ackerbau und in der Milchproduktion wird ein Teil der Mitarbeiter am Betriebszweig- oder Gesamtbetriebsergebnis beteiligt.

\subsubsection{Entlohnungssysteme bei den Führungskräften}

Zwar spielen Führungskräfte in der Landwirtschaft eine zahlenmäßig untergeordnete Rolle, die Entlohnung von Führungskräften ist jedoch nicht nur von praktischer, sondern auch von theoretischer Bedeutung. Die Erkenntnisse aus der PrinzipalAgenten-Theorie (vgl. Kapitel 3.1.1) legen eine Risikobeteiligung der Führungskräfte nahe, um moral hazard zu vermeiden. Tabelle 25 zeigt die in den erhobenen Betrieben verwendeten Entlohnungssysteme für Führungskräfte.

TABELLE 25 : ENTLOHNUNGSSYSTEME BEI DEN FÜHRUNGSKRÄFTEN

\begin{tabular}{|l|c|}
\hline Fester Stundenlohn/Gehalt & $96,7 \%$ \\
\hline Gewinnbeteiligung & $24,4 \%$ \\
\hline Tantiemeregelung & $21,1 \%$ \\
\hline Höhergruppierung in Lohnstufen in Abhängigkeit von der Leistung & $12,2 \%$ \\
\hline Prämien, z.B. für die Entwicklung der Kosten oder der Milchleistung & $11,4 \%$ \\
\hline Kapitalbeteiligung & $4,1 \%$ \\
\hline
\end{tabular}

Quelle: Eigene Erhebung, Betriebsleiterbefragung, Mehrfachantworten möglich, N=123

Die Führungskräfte erhalten in 96,7 \% der analysierten Unternehmen einen festen Stundenlohn bzw. ein Gehalt. Dieser Anteil der Zeitentgelte ist sogar noch höher als in der Produktion. Prämienlöhne spielen hingegen bei der Entlohnung der Führungskräfte eine geringere Rolle als bei den Mitarbeitern in der Produktion, unabhängig von der Produktionsrichtung. 
$12,2 \%$ der befragten Betriebsleiter, die Führungskräfte beschäftigen, nehmen auch bei den Führungskräften eine Höhergruppierung in Lohnstufen in Abhängigkeit von der Leistung vor.

Das Ergebnis spiegelt die Problematik der Lohngestaltung von Führungskräften gut wider. Managementaufgaben umfassen vor allem kognitive Aufgaben, die nicht kardinal messbar sind. Die Einführung von Prämien für bestimmte messbare Teilbereiche des Managements könnte zu so genannten „Spill-over-Effekten“ führen (vgl. Kapitel 3.3.2).

Hingegen lassen sich Führungskräfte leichter am Erfolg beteiligen, da ihr tatsächlicher Einfluss auf den Betriebserfolg größer ist als bei den Arbeitern. So verwundert es auch nicht, dass 24,4\% der befragten Betriebsleiter ihre leitenden Angestellten am Gewinn beteiligen oder wie in $21,1 \%$ der Fälle andere Tantiemeregelungen gefunden wurden.

Eine Beteiligung der Führungskräfte am Kapital des Unternehmens wird jedoch nur bei $4,1 \%$ der analysierten Betriebe durchgeführt.

Neben den oben aufgeführten Antwortmöglichkeiten hatten die Betriebsleiter die Möglichkeit, noch weitere Entlohnungsformen für die von ihnen beschäftigten Führungskräfte zu nennen.

Ein Betriebsleiter leistet in Abhängigkeit vom Betriebsergebnis zusätzliche Beiträge zur Altersversorgung. Auch andere Betriebsleiter gewähren den Führungskräften eine zusätzliche Betriebsrente.

Ein weiterer Betriebsleiter nimmt eine Gehaltsanhebung bei den Führungskräften vor, wenn sich der Betrieb positiv entwickelt. Die Einschätzung der Betriebsentwicklung wird aber nach Aussage des Betriebsleiters auf subjektiver Basis vorgenommen. Auch werden von anderen Betriebsleitern ergebnisabhängige Sonderzahlungen gewährt.

In einem Betrieb erhalten die Führungskräfte, wenn sie einfachere Arbeiten erledigen müssen, hier wurde beispielhaft das Mistfahren angeführt, 1,5€ mehr als ein Arbeiter in der Produktion. Diese Maßnahme lässt sich motivationstheoretisch mit Adams Equity Theorie begründen. Die Führungskraft könnte sich nämlich, vorausgesetzt sie sieht ihre Qualifikation und Position als relevanten Input an, gegenüber den Arbeitern 
benachteiligt fühlen. Dies könnte in eine schlechtere Verrichtung der Arbeit münden, womit interpersonelle Gleichheit wieder hergestellt wäre, aber zugleich die Gesamtproduktivität des Betriebes zurückginge.

\subsubsection{Lohnhöhe in den analysierten Betrieben}

Im folgenden Abschnitt soll die Höhe der Entlohnung in den analysierten Betrieben untersucht werden. Die Tabelle 26 zeigt die durchschnittlichen Bruttostundenlöhne in den analysierten Betrieben.

TABELLE 26: DURCHSCHNITTLICHE BRUTTOLÖHNE (ARBEITNEHMERBRUTTO) IN DEN ANALYSIERTEN BETRIEBEN

\begin{tabular}{|l|c|c|c|c|}
\hline Bereich & $\begin{array}{c}\varnothing \text { Bruttostundenlohn } \\
\text { (ohne Zuschläge) in } \\
\epsilon / h\end{array}$ & $\begin{array}{c}\varnothing \text { Bruttostundenlohn } \\
\text { (mit Zuschlägen) in } \epsilon / h\end{array}$ & $\begin{array}{c}\varnothing \text { Bruttostunden- } \\
\text { lohn (ohne } \\
\text { Zuschläge) in } \epsilon / h \\
\text { ABL }\end{array}$ & $\begin{array}{c}\varnothing \\
\text { Bruttostunden- } \\
\text { lohn (ohne } \\
\text { Zuschläge) in } \epsilon / h \\
\text { NBL }\end{array}$ \\
\hline Leitung & 14,59 & 15,72 & 16,87 & 14,27 \\
\hline Verwaltung & $9,62^{27}$ & 10,38 & 9,7 & 9,63 \\
\hline $\begin{array}{l}\text { Pflanzen- } \\
\text { produktion }\end{array}$ & 8,54 & 9,24 & 10,87 & 8,02 \\
\hline $\begin{array}{l}\text { Tier- } \\
\text { produktion }\end{array}$ & 8,19 & 8,79 & 10,21 & 7,81 \\
\hline
\end{tabular}

Quelle: Eigene Erhebung, Betriebsleiterbefragung, Mehrfachantworten möglich

In der Leitung werden durchschnittlich 14,59 Euro pro Stunde ohne Leistungszuschläge oder andere Gratifikationen gezahlt. Mit Zuschlägen sind es 15,72 Euro. In den alten Bundesländern liegt die Vergütung für die Leitungsebene um durchschnittlich 2,6 Euro höher als in den Betrieben der neuen Bundesländer. Vergleicht man den in der Betriebsführung erzielbaren Lohn mit den in Kapitel 2.2.1 aufgeführten Vergleichslöhne von qualifizierten Arbeitern im Berg- oder Hoch- und Tiefbau, so wird deutlich, dass ein Betriebsleiter in der Landwirtschaft unwesentlich mehr verdient als ein Bauarbeiter. Diese Tatsache ist vor dem Hintergrund eines möglichen Führungskräftemangels in der Landwirtschaft sehr kritisch zu sehen.

In der Verwaltung liegen die durchschnittlichen Löhne inklusive aller Zuschläge durchschnittlich bei 10,38 Euro. Die Bruttolöhne ohne zusätzliche Zuschläge

27 Der durchschnittliche Bruttostundenlohn in der Verwaltung ist geringer als der entsprechende Durchschnitt aus den Kategorien alte und neue Bundesländer, da einige Betriebsleiter keine Angaben zum Bundesland gemacht haben. 
unterscheiden sich zwischen den Betrieben der alten und neuen Bundesländer auf Verwaltungsebene nur wenig.

Dies erklärt sich, wenn man einmal die gezahlten Löhne in der Verwaltung mit den Löhnen in der Pflanzen- und Tierproduktion vergleicht. Es zeigt sich, dass in den alten Bundesländern die mit Verwaltungsaufgaben beschäftigten Personen durchschnittlich weniger verdienen als die Mitarbeiter in der Pflanzen- oder Tierproduktion. In den neuen Bundesländern verdienen die Verwaltungskräfte hingegen mehr Geld als die Arbeiter in den Produktionsbereichen. Möglicherweise sind die Mitarbeiter in der Verwaltung in den alten und neuen Bundesländern mit unterschiedlichen Arbeiten betraut. So könnten in den Betrieben der alten Bundesländer die Tätigkeit in der Verwaltung mehr aus Büroservice- und Schreibtätigkeiten bestehen, während die Verwaltungsmitarbeiter in den neuen Bundesländern stärker mit Controlling-, Rechnungswesen- und Flächenmanagementaufgaben betraut sind. Die Datenlage lässt aber eine abschließende Beurteilung nicht zu.

In der Pflanzenproduktion haben die Mitarbeiter in den analysierten Betrieben ohne Zuschläge durchschnittlich 8,54 Euro erhalten, mit Zuschlägen waren es 9,24 Euro. Durchschnittlich erhalten die Beschäftigten in der Pflanzenproduktion in den alten Bundesländern 2,85 Euro mehr als in den neuen Bundesländern.

Mit einem durchschnittlichen Bruttolohn von 10,87 Euro pro Stunde in den alten Bundesländern liegen die Verdienste in der Pflanzenproduktion auf einem vergleichbaren Niveau wie die in Kapitel 2.2.1 dargestellten durchschnittlich bezahlten Stundenlöhne für Arbeiter in der Landwirtschaft.

Der durchschnittliche Stundenlohn der Mitarbeiter in der Pflanzenproduktion in den neuen Bundesländern liegt hingegen über den in der Agrarstatistik ausgewiesenen durchschnittlichen Stundenlöhnen (vgl. Kapitel 2.2.1).

Die Mitarbeiter der Tierproduktion werden in den analysierten Betrieben unabhängig von der Region am schlechtesten bezahlt. Sie erhalten inklusive aller Zuschläge im Durchschnitt der Betriebe 0,45 Euro weniger als die Mitarbeiter in der Pflanzenproduktion. In den alten Bundesländern beträgt die Lohnschere zwischen den beiden Arbeitsbereichen 0,66 Euro pro Stunde und in den neuen Bundesländern 0,21 
Euro. Die geringen Verdienstmöglichkeiten in der Tierproduktion sind gerade hinsichtlich der erschwerten Arbeitsbedingungen bedenklich. Ein Leiter einer Agrargenossenschaft kommentierte dieses während der Ergebnisworkshops und gab zu bedenken, dass die wirtschaftliche Lage vieler Tierproduktionsbetriebe die Anhebung der Löhne nicht erlaube. Oftmals stünden die Betriebsleiter vor den Alternativen Beibehaltung der Niedriglöhne oder Schließung der Tierproduktion beziehungsweise des Betriebes (Betriebsleiter B).

Ein anderer Agrargenossenschaftsleiter berichtete, dass er zum Zeitpunkt seines Berufseinstiegs den Lohnabstand zwischen Pflanzen- und Tierproduktion ebenfalls als ungerecht ansah und die Lohnhöhe in der Tierproduktion kritisch hinterfragte. Ein Kollege aus der Unternehmensführung riet ihm von einer Angleichung der Stundenlöhne ab. Eine solche Maßnahme würde Unmut bei den Traktoristen nach sich ziehen, da sich diese in der Betriebshierarchie den Mitarbeitern in der Tierproduktion überlegen fühlten. Eine Harmonisierung der Stundenlöhne käme einer Herabwürdigung der innerbetrieblichen Position des Traktoristen gleich.

Die Abbildung 23 zeigt den Schwankungsbereich der bezahlten Bruttostundenlöhne (ohne Leistungszuschläge, Erfolgsbeteiligungen bzw. Dividenden) in Euro pro Stunde in den alten und neuen Bundesländern mit den Minimal-, Durchschnitts- und Maximalwerten. Es wird deutlich, dass die Löhne deutlichen Schwankungen zwischen den Betrieben unterliegen.

\section{ABBILDUNG 23: BRUTTOLÖHNE UND IHR SCHWANKUNGSBEREICH}

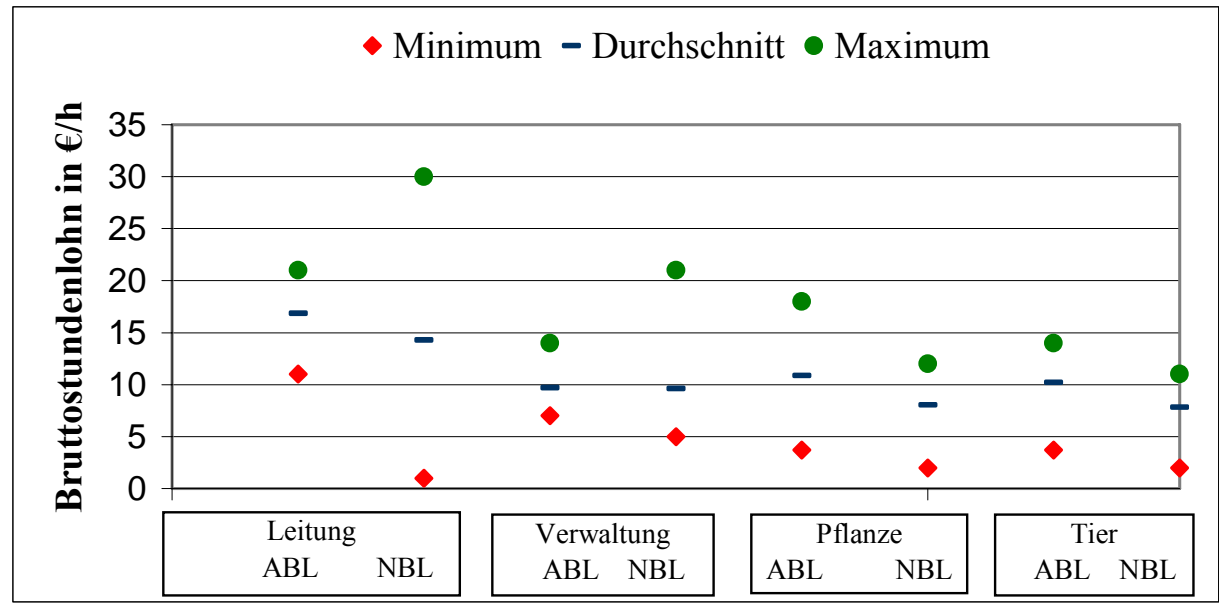

Quelle: Eigene Erhebung, Betriebsleiterbefragung 
Ein Mittelwertvergleich zeigt hoch bzw. höchst signifikante Unterschiede zwischen den Löhnen in der Tier- und Pflanzenproduktion und Betrieben verschiedener Rechtsform. Dieses Ergebnis könnte jedoch durch die Regionszugehörigkeit verzerrt sein, da die juristischen Personen vor allem in den neuen Bundesländern liegen. Insgesamt werden die Mitarbeiter in der Rechtsform der Einzelunternehmen durchschnittlich am höchsten bezahlt. Beschränkt man den Mittelwertvergleich auf die Betriebe in den neuen Bundesländern, so ist das Ergebnis weniger signifikant. In der Pflanzenproduktion werden die Mitarbeiter wieder in den Einzelunternehmen besser bezahlt als in den Personen- oder Kapitalgesellschaften. Es ist aber zu bedenken, dass die Mitarbeiter der Kapitalgesellschaften, die Anteilseigner sind, zusätzliches Einkommen durch Dividenden oder Werteerhöhungen erwirtschaften können. Die Arbeitskräfte in den Personengesellschaften erhalten mehr Lohn als in den juristischen Personen. In der Tierproduktion werden ebenfalls die Mitarbeiter der Einzelunternehmen am höchsten bezahlt, der Lohnunterschied zwischen Personen- und Kapitalgesellschaften ist jedoch nicht eindeutig, da er sehr betriebsabhängig ist. In der Tierproduktion bezahlen die Betriebsleiter der eG durchschnittlich mehr als in $\mathrm{GbR}$ und $\mathrm{GmbH}$, in der Pflanzenproduktion ist der Lohnabstand zu den Personengesellschaften und anderen juristischen Personen nicht besonders groß. Die Nachfolgeunternehmen der LPG haben außerdem gegenüber den wieder eingerichteten Betrieben den Nachteil, dass sie trotz eines zum Teil massiven Personalabbaus immer noch einen überhöhten Personalbestand haben, der sich auch auf die Lohnhöhe in den Betrieben auswirkt.

\subsection{Erfahrungen mit leistungsorientierter Lohngestaltung}

\subsubsection{Erfahrungen der Betriebsleiter}

Sowohl Arbeitgeber als auch Arbeitnehmer wurden zu ihren Erfahrungen mit leistungsorientierter Entlohnung befragt. Von den befragten Arbeitgebern haben 42,9 \% bereits Erfahrungen mit Leistungslöhnen gemacht.

\subsubsection{Ergebnisse der logistischen Regression}

Mit Hilfe verschiedener binärer Logit-Modelle soll der Einfluss unterschiedlicher unabhängiger Variablen auf die abhängige Variable „Erfahrung mit leistungsorientierter Entlohnung“ untersucht werden. Ziel der Logitanalyse ist $\mathrm{zu}$ modellieren, welche 
Variablen die Wahrscheinlichkeit beeinflussen, dass ein Betriebsleiter bereits Erfahrung mit leistungsorientierten Entlohnungssystemen gesammelt hat. Außerdem soll das Modell zeigen, in welche Richtung der Zusammenhang zwischen unabhängiger und abhängiger Variablen gilt.

Die logistische Regression ermöglicht den Einfluss von unabhängigen intervallskalierten, dichotomen und kategorialen (nach Transformation in dichotome Variable) Variablen auf eine dichotome (binär logistische Regression) oder polytom nicht-metrische abhängige Variable (multinomiale logistische Regression) zu messen (DIEKMANN 2004: 614).

Die abhängige Variable, die bei der binär logistischen Regression in der Regel die Werte „0“ oder „1“ hat, wird durch logarithmieren und weitere Umformungen so transformiert, dass sie einen theoretischen Wertebereich $-\infty$ bis $+\infty$ annehmen kann. Als abhängige Größe wird die Wahrscheinlichkeit des Übergangs zur Referenzgruppe $\mathrm{p}\left(\mathrm{y}_{\mathrm{i}}=1\right)$ betrachtet. SPSS sieht als Referenzgruppe immer die Gruppe mit der höchsten Codierung an. Im hier vorliegenden Logit-Modell ist die Zustimmung zur Frage „Haben Sie bereits Erfahrung mit leistungsorientierter Entlohnung gesammelt?“ die Referenzgröße.

Die Schätzung der $\beta$-Koeffizienten zur Trennung der zwei Ausprägungen der abhängigen Variablen wird mit der Maximum-Likelihood-Methode vorgenommen.

Im Untersuchungsmodell der empirischen Analyse wurde angenommen, dass verschiedene Einflussgrößen die Eignung bzw. die Wahl eines Anreizsystems begründen können.

Im Folgenden wird geprüft, welchen Einfluss verschiedene Variablen auf die Erfahrung mit leistungsorientierter Entlohnung haben. Hierzu zählen:

- Flächenausstattung und Tierbestände,

- Anzahl der Arbeitskräfte,

- Produktionsrichtung und -weise, Rechtsform, 
- Mitarbeitermotivation,

- Betriebsleitereigenschaften und -einstellungen,

- und soziodemographische Angaben der Betriebsleiter.

Die Logit-Modelle wurden durch die zweistufige Eliminierung von Ausreißern verbessert, deren Pearson-Residuen sich um mehr als zwei Standardabweichungen von den anderen Fällen unterschieden (FROMM 2005: 27).

Zunächst wird der Einfluss unterschiedlicher Betriebsgrößenkennzahlen auf die Erfahrung der Betriebsleiter mit leistungsorientierter Entlohnung untersucht. Die dazugehörigen Logit-Modelle sind in Tabelle 27 dargestellt.

Das Modell „Milchkühe und Acker“ zeigt, dass die Wahrscheinlichkeit, dass ein Betriebsleiter bereits Erfahrung mit leistungsorientierter Entlohnung gesammelt hat, steigt, wenn

- die Anzahl der Milchkühe und Rinder zunimmt und

- die Gesamtfläche des Betriebes steigt.

Hingegen sinkt die Wahrscheinlichkeit der Erfahrung mit leistungsorientierter Entlohnung mit steigender Ackerfläche.

Auch in Schweine haltenden Betrieben steigt die Wahrscheinlichkeit der Erfahrung mit leistungsorientierter Entlohnung mit steigender Bestandesgröße und Gesamtfläche des Betriebes. Der Einfluss der Größe des Mastschweinebestandes ist sogar hoch signifikant. Analog zum Modell „Milchkühe und Acker“ hat eine zunehmende Ackerfläche eine senkende Wirkung auf die Wahrscheinlichkeit mit leistungsorientierter Entlohnung. 
TABELLE 27: EINFLUSS DER BETRIEBS- UND BESTANDESGRÖßEN AUF DIE ERFAHRUNG MIT LEISTUNGSORIENTIERTER ENTLOHNUNG

\begin{tabular}{|c|c|c|}
\hline & $\begin{array}{c}\text { Modell „, Milchkühe und Acker“ } \\
n=135\end{array}$ & $\begin{array}{c}\text { Modell „Acker und Schweine“ } \\
n=32\end{array}$ \\
\hline & \multicolumn{2}{|c|}{ Regressionskoeffizienten $\beta$} \\
\hline Konstante & $-1,668 * * *$ & $-3,813 *$ \\
\hline Anzahl Milchkühe & ,002 & \\
\hline Anzahl Rinder & 001 & \\
\hline Mastschweineplätze & & $0,002^{* *}$ \\
\hline Zuchtsauen & & 0,002 \\
\hline Ackerfläche in ha &,$- 003 *$ & $-0,009^{*}$ \\
\hline Gesamtfläche in ha &, $003 * *$ &, $007 *$ \\
\hline Cox-Snell-Pseudo $r^{2}$ : & 0,223 & 0,478 \\
\hline Nagelkerkes $r^{2}$ : & 0,298 & 0,638 \\
\hline Prozentsatz der Richtigen & $72,6 \%$ & $81,3 \%$ \\
\hline
\end{tabular}

Quelle: Eigene Erhebung, Betriebsleiterbefragung, ohne Ausreißer

Die Güte der Modellanpassung bewegt sich mit Pseudo-r²-Werten zwischen 0,223 und 0,298 im Modell „Acker und Milchkühe“ und 0,478 und 0,638 im Modell „Acker und Milchkühe“ im akzeptablen bis guten Bereich (DIAZ-BONE UND KÜNEMUND 2003: 13). Dies verdeutlicht auch der gute Prognoseerfolg des Modells. Das erste Modell ordnet mit Hilfe der ausgewiesenen Parameter die Betriebsleiter zu 72,6 \% der richtigen Gruppe, das zweite Modell mit 81,3\%.

Die betriebswirtschaftliche Ausrichtung des Betriebes liefert keinen bedeutenden Erklärungsbeitrag zur Prognose der Wahrscheinlichkeit einer leistungsorientierten Entlohnung, aus diesem Grunde ist das Logit-Modell nicht aufgeführt. Dieses Ergebnis 
ist durchaus konsistent mit den obigen Modellen, die deutlich machen, dass die Betriebs- bzw. Bestandsgröße eine entscheidende Variable ist. Da in der betriebswirtschaftlichen Ausrichtung jedoch Betriebe mit unterschiedlichen Größen enthalten sind, liefert diese Variable keinen Beitrag zur Erklärung des Sachverhalts. Auch die soziodemographischen Variablen wie Bildungsgrad, Alter und Geschlecht des Betriebsleiters liefern keinen signifikanten und deutlichen Betrag auf die abhängige Variable.

Hingegen erhöht die Zugehörigkeit zur Rechtsform juristischer Personen die Wahrscheinlichkeit, dass der Betriebsleiter schon einmal Erfahrung mit leistungsorientierter Entlohnung gesammelt hat.

\section{Betriebsleitereigenschaften}

Wie Tabelle 28 zeigt, kann ein Teil der Varianz der Zustimmung zu den Erfahrungen mit leistungsorientierter Entlohnung durch weitere unabhängige Variablen erklärt werden. Hierzu wurden verschiedene Variablen schrittweise in das Modell aufgenommen. Es wurde der Algorithmus „Vorwärts schrittweise Likelihood-Quotient“ verwendet. D.h., es wird in jedem Schritt (nach Aufnahme der Konstanten) eine Variable aufgenommen, die den größten Teil der Varianz erklärt und das Wahrscheinlichkeitsverhältnis maximiert.

\section{TABELLE 28: BINÄRES LOGIT-MODELL ZUR ERFAHRUNG MIT LEISTUNGSORIENTIERTER ENTLOHNUNG}

\begin{tabular}{|c|c|c|}
\hline & Regressionskoeffizient $\beta$ & Effekt-Koeffizient $(\operatorname{Exp}(\beta))$ \\
\hline Konstante & $-0,703^{* *}$ & 0,495 \\
\hline Anzahl Fremdarbeitskräfte & $0,032 * *$ & 1,033 \\
\hline $\begin{array}{l}\text { „Gute Leistung honorieren } \\
\text { wir immer finanziell"“ }\end{array}$ & $0,528^{*}$ & 1,696 \\
\hline $\begin{array}{l}\text { „Leistungslohnsysteme } \\
\text { brauche ich in meinem } \\
\text { Betrieb nicht, da er } \\
\text { übersichtlich strukturiert } \\
\text { ist.“ }\end{array}$ & $-1,046^{* * *}$ & 0,351 \\
\hline $\begin{array}{l}\text { „Lob und Tadel haben } \\
\text { einen größeren } \\
\text { Motivationseffekt als das } \\
\text { Vergütungssystem.“" }\end{array}$ & $-0,613^{*}$ & 0,542 \\
\hline
\end{tabular}

Quelle: Eigene Berechnung, n=192, ohne Ausreißer 
In das Logit-Modell fließen 192 Fälle ein. Die Reduzierung im Vergleich zu den 260 Datensätzen, die aus der Befragung vorliegen, resultiert aus den Fällen mit fehlenden Werten sowie aus dem Ausschluss der Ausreißer.

Das binäre Logit-Modell zeigt, dass die Wahrscheinlichkeit, bereits Erfahrung mit leistungsorientierter Entlohnung gesammelt zu haben, mit der Anzahl der beschäftigten Lohnarbeitskräfte steigt. Der Zusammenhang ist hoch signifikant.

Von großer Bedeutung scheint die Einstellung des Betriebsleiters zu verschiedenen Formen der Mitarbeitermotivation zu sein. Umso mehr ein Betriebsleiter dem Statement „Gute Leistung belohnen wir immer finanziell“" zustimmt, desto wahrscheinlicher ist es, dass er bereits Erfahrung mit Leistungslohnsystemen gemacht hat. Die Variable könnte eine Tautologie zur abhängigen Variablen darstellen. Ein Betriebsleiter, der gute Leistung immer finanziell belohnt, muss aber nicht zwangsläufig durch Prämienlöhne oder Leistungszulagen entlohnen, er könnte dieses auch durch Effizienzlöhne ausdrücken oder nach Leistung befördern. Bei Betriebsleitern, die der Meinung sind, dass immaterielle Anreize, wie Lob oder Tadel, einen größeren Einfluss auf die Mitarbeitermotivation als materielle Anreize haben, ist die Wahrscheinlichkeit geringer, dass sie leistungsorientiert entlohnen.

Offensichtlich hat die Komplexität der Betriebsorganisation einen höchst signifikanten Einfluss auf die abhängige Variable. Die Wahrscheinlichkeit einer an die Leistung gebundenen Vergütung sinkt mit der Zustimmung zum Statement „Leistungslohnsysteme brauche ich in meinem Betrieb nicht, da er übersichtlich strukturiert ist.“

Als Konsequenz aus den Ergebnissen der logistischen Regression lässt sich ableiten, dass für die Wahrscheinlichkeit der Erfahrung mit leistungsorientierter Entlohnung weniger die Mitarbeitermotivation als die Einstellung der Betriebsleiter zu Anreizsystemen von Bedeutung ist. Das Ergebnis spiegelt die kontroversen Aussagen der verschiedenen Theorien zur Gestaltung von Anreizsystemen wider. So wie es in den unterschiedlichen ökonomischen Strömungen Anhänger und Gegner materieller Anreize gibt, gibt es auch Betriebsleiter, die diese befürworten oder ablehnen. Dementsprechend neigen Betriebsleiter, die positiv zu finanziellen Anreizen eingestellt sind, eher zur 
leistungsorientierten Entlohnung als Betriebsleiter, die immaterielle Anreize befürworten.

Die in der Tabelle 28 ausgewiesenen Gütemaße für die Modellanpassung, das CoxSnell-pseudo- ${ }^{2}$ und das Nagelkerke-r ${ }^{2}$, liegen bei 0,317 bzw. 0,428. Wie bereits im ersten Modell erwähnt, deutet dies auf eine akzeptable Modellanpassung hin. Dass jedoch kein besserer Wert erreicht wird, deutet darauf hin, dass in der Befragung Variablen nicht erfasst wurden, die die abhängige Variable noch besser erklären würden. Möglicherweise liegen diese im Bereich der Betriebsleiterpersönlichkeit, und hier im Besonderen im Führungsstil. Ein Indiz für die Bedeutung der Führungsqualität kann in zwei schriftlichen Kommentaren der befragten Betriebsleiter gesehen werden. Als Notiz neben der Frage, ob die Betriebsleiter bereits Erfahrung mit leistungsorientierter Entlohnung gesammelt hätten, wurden die folgenden Anmerkungen gemacht:

Betriebsleiter C: „Ja, da aber heutzutage Erfolge oder Misserfolge Ausdruck der Leitung sind, haben wir solche Systeme nicht in Anwendung“".

Betriebsleiter D: „Marx sagt: „Wie die Leitung, so die Leistung““

Offensichtlich sind Führungsstile ein weiteres wichtiges Forschungsfeld des Personalmanagements in der Landwirtschaft, das aber in dieser Arbeit nicht weiter behandelt werden kann.

\subsubsection{Clusteranalyse zu den Betriebsleitererfahrungen mit Leistungslöhnen}

In einem weiteren Schritt wurden die Betriebsleiter, die Aussagen zur leistungsorientierten Entlohnung gemacht hatten, mit Hilfe einer Clusteranalyse gruppiert. Das Ziel ist es, Informationen darüber zu erhalten, wie sich die Erfahrungen der Betriebsleiter in Bezug auf Leistungslöhne unterscheiden, und Einflussgrößen auf ein unterschiedliches Antwortverhalten herauszuarbeiten, um daraus Anwendungsempfehlungen für diese Entlohnungsform ableiten zu können.

Die gebildeten Gruppen sollen in sich homogen und untereinander möglichst heterogen sein. Voraussetzung für die Clusterbildung ist die Unkorreliertheit der in die Analyse 
eingehenden Faktoren. Zu diesem Zwecke wurden die Statements zu den Erfahrungen mit leistungsorientierter Entlohnung zunächst einer Faktorenanalyse unterzogen.

Es konnte zwar eine 4-Faktorlösung gefunden werden, die letzten beiden Faktoren beinhalteten jedoch nur je ein Item. Aus diesem Grunde wurde auf die Einbeziehung der Faktoren als clusterbildende Variablen verzichtet und es wurden vier unkorrelierte Items zur Clusterbildung verwendet.

Zur Eliminierung von Ausreißern wurde zunächst die Methode des nächstgelegenen Nachbars verwendet. Die Clusterbildung erfolgt mit der Ward-Methode, die zu einer plausiblen 3-Clusterlösung führte, die die 88 Nennungen verhältnismäßig gleichmäßig verteilt. Die Tabelle 29 zeigt, in welchen Punkten sich die Cluster in den clusterbildenden Variablen unterscheiden:

TABELLE 29: CLUSTERBESCHREIBUNG

\begin{tabular}{|c|c|c|c|c|}
\hline & $\begin{array}{c}\text { Cluster } 1 \\
n=33\end{array}$ & $\begin{array}{c}\text { Cluster } 2 \\
n=24\end{array}$ & $\begin{array}{c}\text { Cluster } 3 \\
n=31\end{array}$ & $\begin{array}{c}\text { Skalierung } \\
\text { der Variable }\end{array}$ \\
\hline \multicolumn{5}{|c|}{ Clusterbildende Variablen } \\
\hline $\begin{array}{l}\text { „Leistungsorientierte } \\
\text { Entlohnungssysteme führen } \mathrm{zu} \text { einer } \\
\text { besseren Motivation meiner } \\
\text { Mitarbeiter.“* }\end{array}$ & $\begin{array}{c}0,91 \\
(\sigma=0,631)\end{array}$ & $\begin{array}{c}1 \\
(\sigma=0,722)\end{array}$ & $\begin{array}{c}0,61 \\
(\sigma=0,761)\end{array}$ & \multirow{4}{*}{ 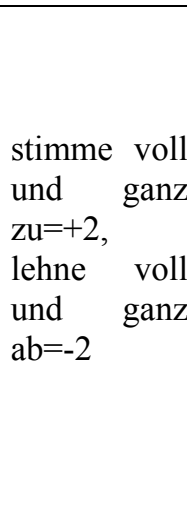 } \\
\hline $\begin{array}{l}\text { „Durch das leistungsorientierte } \\
\text { Entlohnungssystem fühlen sich meine } \\
\text { Mitarbeiter zu sehr kontrolliert.“**** }\end{array}$ & $\begin{array}{c}-1,12 \\
(\sigma=0,331)\end{array}$ & $\begin{array}{c}0,25 \\
(\sigma=0,442)\end{array}$ & $\begin{array}{c}-0,87 \\
(\sigma=0,670)\end{array}$ & \\
\hline $\begin{array}{l}\text { „Leistungsbeurteilungen setzen klare } \\
\text { Maßstäbe voraus.“* }\end{array}$ & $\begin{array}{c}1,24 \\
(\sigma=0,561)\end{array}$ & $\begin{array}{c}1 \\
(\sigma=0,417)\end{array}$ & $\begin{array}{c}1,32 \\
(\sigma=0,475)\end{array}$ & \\
\hline $\begin{array}{l}\text { „Ich achte darauf, dass die } \\
\text { Einkommensunterschiede } \\
\text { meinen Mitarbeitern nicht } \mathrm{zu} \text { hoch } \\
\text { werden.“*** }\end{array}$ & $\begin{array}{c}1,06 \\
(\sigma=0,348)\end{array}$ & $\begin{array}{c}0,38 \\
(\sigma=0,770)\end{array}$ & $\begin{array}{c}-0,74 \\
(\sigma=0,682)\end{array}$ & \\
\hline
\end{tabular}

Quelle: Eigene Erhebung

\section{Cluster 1: „Uneingeschränkte Befürworter“ $(n=33)$}

Das erste Cluster lässt sich mit der Bezeichnung „Uneingeschränkte Befürworter“ leistungsorientierter Entlohnungssysteme beschreiben. Die in diesem Cluster enthaltenen Betriebsleiter sind überwiegend der Meinung, dass leistungsorientierte Entlohnungssysteme zu einer besseren Motivation ihrer Mitarbeiter führen. Sie lehnen in hohem Maße das Statement ab, dass leistungsorientierte Entlohnungssysteme von den Mitarbeitern als starke Fremdkontrolle empfunden werden könnte. Diese Betriebsleiter 
sind in hohem Maße der Meinung, dass klare Maßstäbe bei der Lohnfindung eine wichtige Voraussetzung für leistungsorientierte Entgeltsysteme sind, und achten darauf, dass zwischen den Mitarbeitern die Einkommensunterschiede nicht zu groß werden. Diese Antwort mag zunächst nicht plausibel erscheinen, denn wenn ein Betriebsleiter leistungsabhängig belohnt, führt dies automatisch $\mathrm{zu}$ Einkommensunterschieden zwischen den Mitarbeiter. Der Anspruch der Lohngerechtigkeit als wichtiger Erfolgsbedingung für leistungsorientierte Entlohnung beinhaltet aber, dass die Einkommensunterschiede durch die unterschiedliche Leistung erklärbar sind. Nur wenn das Verhältnis von Input $\mathrm{zu}$ Output im Vergleich $\mathrm{zu}$ einer Referenzperson nachvollziehbar ist, wird das Entlohnungssystem als fair wahrgenommen und kann auch hohe Motivation induzieren. Große Einkommensunterschiede können zum Beispiel zwischen verschiedenen Hierarchieebenen entstehen. Eine starke Überbezahlung von Führungskräften wäre zum Beispiel ein Anlass für Ungerechtigkeitsempfinden zwischen den Mitarbeitern.

\section{Cluster 2: „Kritische Befürworter“6 $(n=24)$}

Das Cluster 2 ist durch eine ambivalente Einstellung zur leistungsorientierten Entlohnung geprägt. Zwar ist hier die größte Zustimmung zur positiven Motivationswirkung dieser Entlohnungsform zu finden. Die zum zweiten Cluster gehörenden Betriebsleiter sind sich jedoch der möglichen Nachteile leistungsorientierter Entlohnung durchaus bewusst. Von den anderen beiden Clustern unterscheidet sich diese Gruppe vor allem dadurch, dass sie dem Statement „Wahrnehmung von Fremdkontrolle“ eher zustimmen. Wie im Cluster 1 ist nach Ansicht der Betriebsleiter des zweiten Clusters die Formulierung klarer Maßstäbe für den Erfolg des leistungsorientierten Lohnsystems von Bedeutung.

\section{Cluster 3: „Lohndifferenzierer“ $(n=31)$}

Im Vergleich zu den ersten beiden Clustern stimmen die Betriebsleiter des Clusters 3 einer positiven Wirkung leistungsorientierter Entlohnung am wenigsten zu. In Bezug auf die möglichen Nachteile leistungsorientierter Entlohnung gleichen sie in ihrem Antwortverhalten stark dem Cluster 1. Die Betriebsleiter in Cluster 1 und 2 stimmten dem Statement „Ich achte darauf, dass Einkommensunterschiede zwischen meinen Mitarbeitern nicht zu groß werden“ zu. Die zum dritten Cluster gehörenden Landwirte 
lehnen diese Aussage eher ab. Cluster 3 zeigt die höchste Zustimmung zur Notwendigkeit klarer Maßstäbe bei der Lohngestaltung.

Im Folgenden soll beleuchtet werden, wie sich die Cluster in Bezug auf weitere mögliche Einflussgrößen unterscheiden. Zunächst sollen die betriebsstrukturellen Unterschiede dargelegt werden (Tabelle 30).

TABELLE 30: BETRIEBSSTRUKTUR DER CLUSTER

\begin{tabular}{|l|c|c|c|}
\hline & $\begin{array}{c}\text { Uneingeschränkte } \\
\text { Befürworter } \\
n=33\end{array}$ & $\begin{array}{c}\text { Kritische Befürworter } \\
n=24\end{array}$ & $\begin{array}{c}\text { Lohndifferenzierer } \\
n=31\end{array}$ \\
\hline $\begin{array}{l}\text { Durchschnittliche } \\
\text { Ackerfläche* }\end{array}$ & 988,58 & 928,00 & 1375,48 \\
\hline $\begin{array}{l}\text { Durchschnittliche } \\
\text { Anzahl Milchkühe* }\end{array}$ & 316 & 280 & 549 \\
\hline $\begin{array}{l}\text { Durchschnittlicher } \\
\text { Anteil Pachtfläche an } \\
\text { Gesamtfläche** }\end{array}$ & $76,9 \%$ & $65,31 \%$ & $85,09 \%$ \\
\hline Fremdarbeitskräfte & 18,13 & 14,25 & 29,33 \\
\hline $\begin{array}{l}\text { Anteil Standort ABL } \\
\text { des Betriebes** }\end{array}$ & $9,7 \%$ & $34,8 \%$ & $10 \%$ \\
\hline $\begin{array}{l}\text { Wirtschaftsweise } \\
\text { (Anteil ökologische } \\
\text { Wirtschaftsweise in } \\
\text { Prozent)* }\end{array}$ & $0 \%$ & $20,8 \%$ & $3,2 \%$ \\
\hline \multicolumn{2}{|l|}{ Signifikanzniveaus: ***: $\mathrm{p}<0,001, * *: \mathrm{p}<0,05, *: \mathrm{p}:<0,1$} \\
\hline
\end{tabular}

Quelle: Eigene Erhebung, Betriebsleiterbefragung

Die Cluster unterscheiden sich deutlich hinsichtlich der Betriebstruktur. Es sind nur die betriebsstrukturellen Parameter ausgewiesen, die einen signifikanten Einfluss auf das Antwortverhalten haben und von denen innerhalb der Cluster eine geringe Anzahl fehlender Werte vorlagen.

Die zum Cluster 1 gehörenden Betriebsleiter, die sich durch eine uneingeschränkte Befürwortung leistungsorientierter Entlohnungssysteme auszeichnen, bewirtschaften in Bezug auf die Ackerfläche, Milchkühe und Fremdarbeitskräfte größere Betriebe als die Betriebsleiter des zweiten Clusters. Im Vergleich zum dritten Cluster sind die Betriebe um fast $30 \%$ kleiner hinsichtlich der durchschnittlichen Ackerfläche und es werden auch nur $57 \%$ der Milchkühe des Clusters 3 gehalten.

Der Pachtanteil unterscheidet sich ebenfalls zwischen den Clustern. Er liegt in Cluster 2 am niedrigsten, gefolgt von Cluster 1 und Cluster 3. Dieses Ergebnis ist konsistent mit der Betriebsgröße und dem Standort der untersuchten Betriebe. Hier unterscheidet sich 
Cluster 2 erheblich von den anderen Clustern: Fast $35 \%$ der Betriebe, deren Leiter zu den kritischen Befürwortern gehören, liegen in den alten Bundesländern.

Ein weiterer interessanter, signifikanter Unterschied zwischen den drei Clustern liegt in der Wirtschaftsweise. 20,8 \% der zu Cluster 2 gehörenden Betriebsleiter bewirtschaften ihren Betrieb ökologisch. Von den ökologisch wirtschaftenden Betriebsleitern gehören 83,3 \% dem Cluster 2 an. Möglicherweise zeichnet sich hier eine unterschiedliche Mentalität der Betriebsleiter von ökologischen und konventionellen Betrieben ab.

Aus den betriebsstrukturellen Unterschieden wird deutlich, dass die Betriebsleiter, die kleinere Betriebe bewirtschaften, eher $\mathrm{zu}$ den Befürwortern dieses Lohnsystems gehören als die Betriebsleiter von Großbetrieben. Die Zuordnung als kleinerer oder größerer Betrieb ist jedoch relativ zu bewerten, denn im statistischen Durchschnitt muss man die analysierten Betriebe ungeachtet der Clusterzugehörigkeit als landwirtschaftliche Großbetriebe bzw. Agrarunternehmen bezeichnen.

\section{ABBILDUNG 24: RECHTSFORMUNTERSCHIEDE ZWISCHEN DEN CLUSTERN}

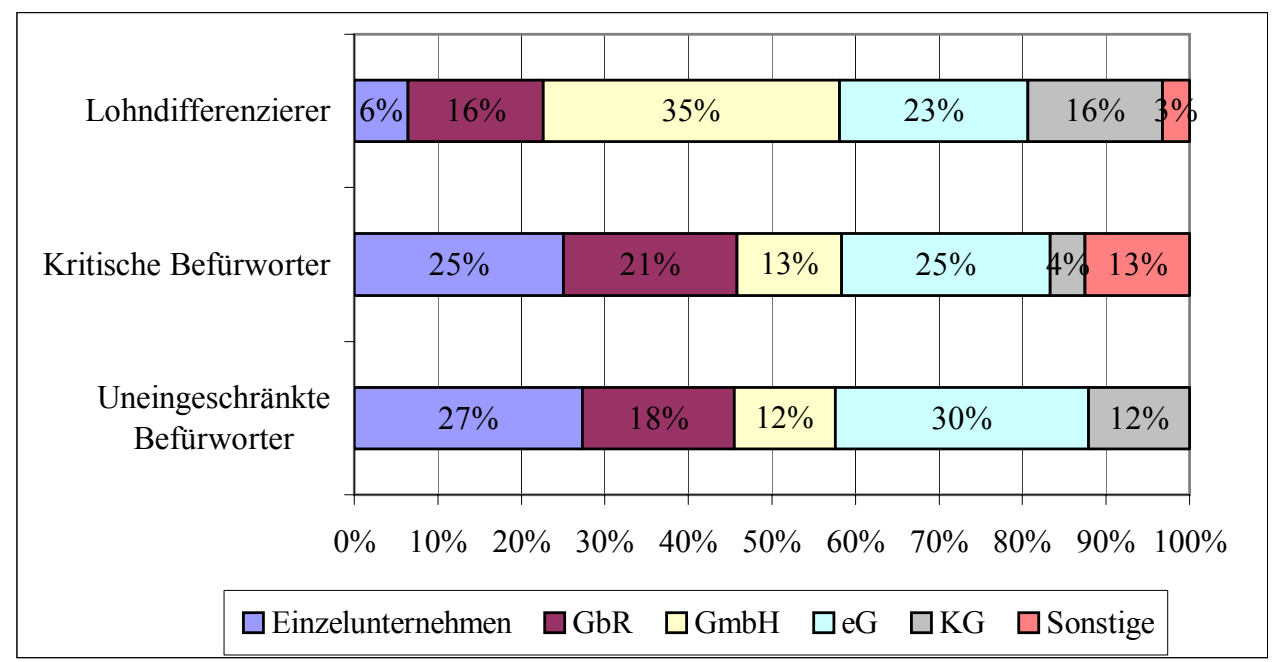

Quelle: Eigene Erhebung, Betriebsleiterbefragung

Abbildung 24 zeigt die relativen Häufigkeiten der verschiedenen Rechtsformen in den untersuchten Clustern. Sie spiegelt die unterschiedlichen Betriebsgrößen in den Clustern wider.

Die Cluster der Befürworter leistungsorientierter Entlohnung (Cluster 1 und 2), sind überwiegend von Betriebsleitern der Rechtsform Einzelunternehmen und 
Personengesellschaften geprägt. Die Einzelunternehmen sind am häufigsten im Cluster vertreten, gefolgt von Cluster 2. Auch die GbR findet sich vermehrt in den befürwortenden Clustern.

Es wird deutlich, dass die juristischen Personen im Cluster Lohndifferenzierer einen bedeutenderen Anteil als in den anderen beiden Clustern haben. Die Einzelunternehmen spielen in diesem Cluster die geringste Rolle. Im dritten Cluster sind vor allem die Betriebsleiter juristischer Personen enthalten. Dementsprechend beschäftigen sie auch im Durchschnitt mehr Lohnarbeitskräfte als die anderen Cluster. Betriebsleiter, die aufgrund des Vorhandenseins verschiedener Hierarchieebenen die Löhne differenzieren müssen, versuchen vielleicht durch eine transparente Lohnfindung potentiellem Ungerechtigkeitsempfinden unter den Mitarbeitern entgegenzuwirken. Es ist hinzuzufügen, dass die Betriebsleiter der Agrargenossenschaften wahrscheinlich über die längste Erfahrung mit leistungsorientierter Entlohnung verfügen. Die Rechtsform hat außerdem einen Einfluss auf die Möglichkeit, ein solches Entgeltsystem überhaupt zu implementieren. Ein befragter Geschäftsführer einer Agrargenossenschaft merkte an, dass die Akzeptanz von Leistungslöhnen im Aufsichtsrat der Genossenschaft nicht gegeben ist und diese Systeme auf Wunsch des Aufsichtsrates abgeschafft wurden. Dies verdeutlicht den Einfluss des Rechts auf unternehmerische Mitbestimmung der Mitarbeiter (vgl. Kapitel 2.3.2.2) auf die Gestaltung von Entlohnungssystemen. 


\section{ABBILDUNG 25: BETRIEBSLEITEREINSTELLUNGEN ZU ANREIZSYSTEMEN}

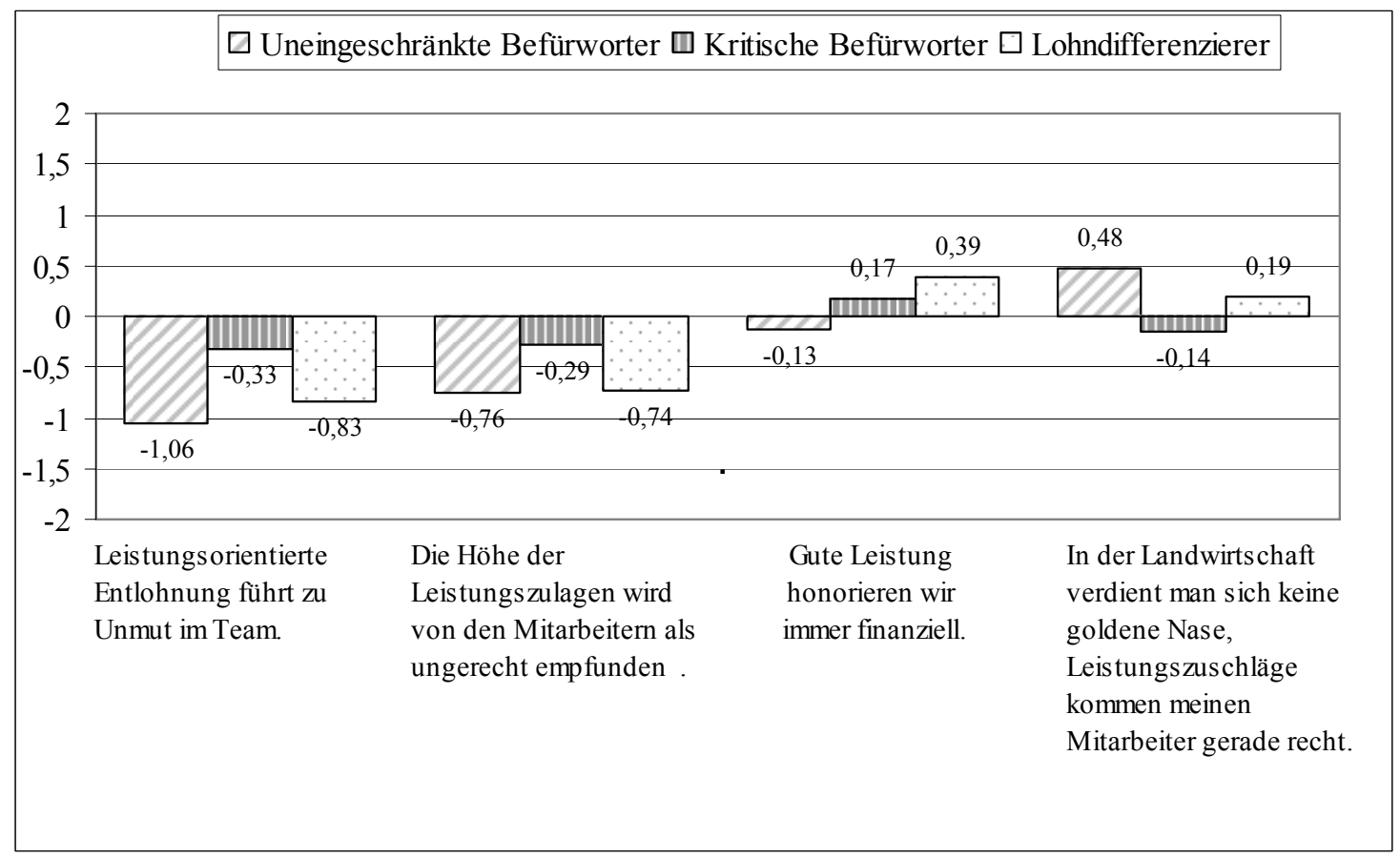

Quelle: Eigene Erhebung, Betriebsleiterbefragung

Abbildung 25 veranschaulicht die Unterschiede im Antwortverhalten der Betriebsleiter $\mathrm{zu}$ verschiedenen Statements zur Gestaltung von Anreizsystemen. Es wurden nur Statements dargestellt, in denen sich die Cluster signifikant voneinander unterscheiden. Es zeigt sich wieder, das Cluster 2 am kritischsten hinsichtlich der Nachteile leistungsorientierter Entlohnungssysteme ist. Zwar lehnen die Betriebsleiter dieses Clusters die Statements „Leistungsorientierte Entlohnung führt zu Unmut im Team“ und „Die Höhe der Leistungszulagen wird von den Mitarbeitern als ungerecht empfunden“ ab, die Ablehnung ist im Vergleich zu den anderen beiden Clustern nur schwach. Folglich stimmen diese Betriebsleiter den möglichen negativen Wirkungen von Leistungslöhnen auf das Betriebsklima und die Lohngerechtigkeit am ehesten zu. Interessanterweise stimmt dieses Cluster dem Statement „Gute Leistung belohnen wir immer finanziell““ eher zu, während Cluster 1, das leistungsorientierte Entlohnung uneingeschränkt befürwortet, das gleiche Statement leicht ablehnt. Die Betriebsleiter des ersten Clusters honorieren gute Leistungen trotz der nach ihrer Meinung guten Motivationswirkung von Leistungslöhnen nicht nur finanziell. Die Wertschätzung finanzieller Anreize durch die Mitarbeiter wird von den Betriebsleitern unterschiedlich gesehen. Cluster 1 und Cluster 3 stimmen eher $\mathrm{zu}$ und Cluster 2 eher ab. Die 
Betriebsleiter des zweiten Clusters zahlen auch durchschnittlich die höchsten Bruttostundenlöhne in Pflanzen- und Tierproduktion. Die Unterschiede im Lohnniveau sind zwischen den Clustern jedoch nicht signifikant.

Aus den Ergebnissen der Clusteranalyse lässt sich das folgende Zwischenfazit ziehen: Die positive Motivationswirkung von Leistungslöhnen wird von den Betriebsleitern insgesamt bestätigt. Die Leiter der besonders großen Betriebe der Stichprobe beurteilen die Motivationswirkung jedoch am kritischsten. Dieses ist die Gruppe der Betriebsleiter, die am häufigsten Erfahrung mit Leistungslöhnen gemacht haben. Negative Wirkung auf das Betriebsklima beobachten die Betriebsleiter eher nicht, das gleiche gilt für ein mögliches Ungerechtigkeitsempfinden in Bezug auf die Höhe der Leistungszulagen. Motivationsdefizite, die durch ein Ungerechtigkeitsempfinden der Mitarbeiter hervorgerufen werden, sind also tendenziell nicht zu beobachten. Die Ausführungen zum Verdrängungseffekt haben gezeigt, dass die Wahrnehmung von Fremdkontrolle durch leistungsorientierte Entlohnung eine positive Motivationswirkung verdrängen kann. Die Betriebsleiter antworten hier in unterschiedliche Richtungen. Die Betriebsleiter, die zum insgesamt kritischsten Cluster hinsichtlich der möglichen Nachteile dieser Entlohnungsform gehören, stimmen hier eher zu. Differenzierte Handlungsempfehlungen für die Leistungsentlohnung in unterschiedlichen Rechtsformen lassen sich aus den Ergebnissen der Clusteranalyse nicht direkt ableiten. Wie die logistische Regression zeigt auch die Clusteranalyse, dass sich die Betriebsleiter in ihrer Einstellung $\mathrm{zu}$ Leistungslöhnen unterschieden. Für die Großbetriebe lässt sich festhalten, dass in diesen die Erfüllung der notwendigen Erfolgsbedingungen für leistungsorientierte Entlohnungssysteme die größte Herausforderung stellt.

\subsubsection{Erfahrungen der Arbeitnehmer mit leistungsorientierter Entlohnung}

Nachdem die Einstellung der Betriebsleiter zur leistungsorientierten Entlohnung dargelegt wurde, ist es interessant, die Perspektive der Mitarbeiter zu betrachten.

$33 \%$ der befragten Arbeitnehmer haben bereits Erfahrungen mit leistungsorientierter Entlohnung gesammelt. 64,2 \% wurden noch nicht nach ihrer Leistung entlohnt und 2,4 \% der Mitarbeiter haben keine Angaben zu dieser Frage gemacht. 
Die Abbildung 26 zeigt, dass die Mitarbeiter, die bereits Erfahrungen mit leistungsorientierter Entlohnung gesammelt haben, ganz überwiegend zufrieden mit dieser Entlohnungsart sind. Knapp $27 \%$ dieser Mitarbeiter sind unentschlossen und $2,4 \%$ nur teilweise damit zufrieden.

\section{ABBILDUNG 26: ZUFRIEDENHEIT DER MITARBEITER MIT LEISTUNGSLÖHNEN}

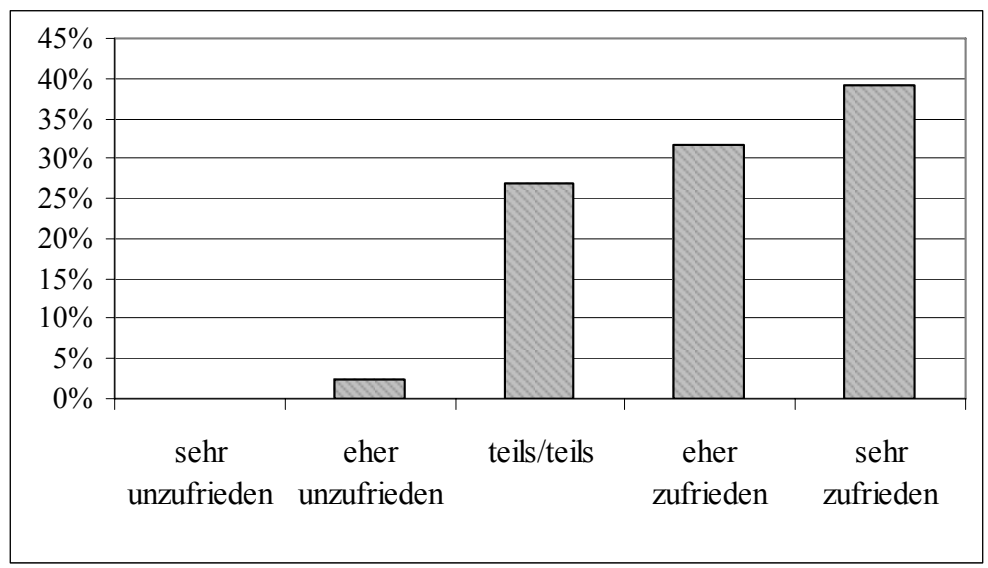

Quelle: Eigene Erhebung, Mitarbeiterbefragung, $\mathrm{N}=41$

Die Motivationswirkung von Leistungslöhnen auf die befragten Mitarbeiter ist ebenfalls positiv (vgl. Abbildung 27). Rund $73 \%$ der gültigen Antworten entfallen auf die

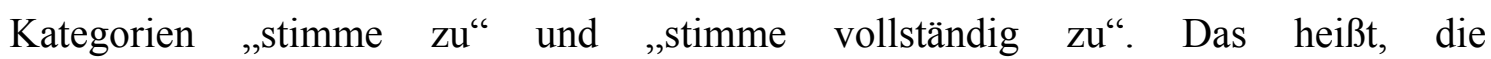
Mitarbeiterbefragung zeigt ein ähnliches Bild wie die Betriebsleiterbefragung hinsichtlich einer positiven Motivationswirkung leistungsorientierter Entlohnungssysteme. 
ABBILDUNG 27: „ZULAGEN, DIE ICH FÜR MEINE LEISTUNG ERHALTE, ERHÖHEN MEINE MOTIVATION“"

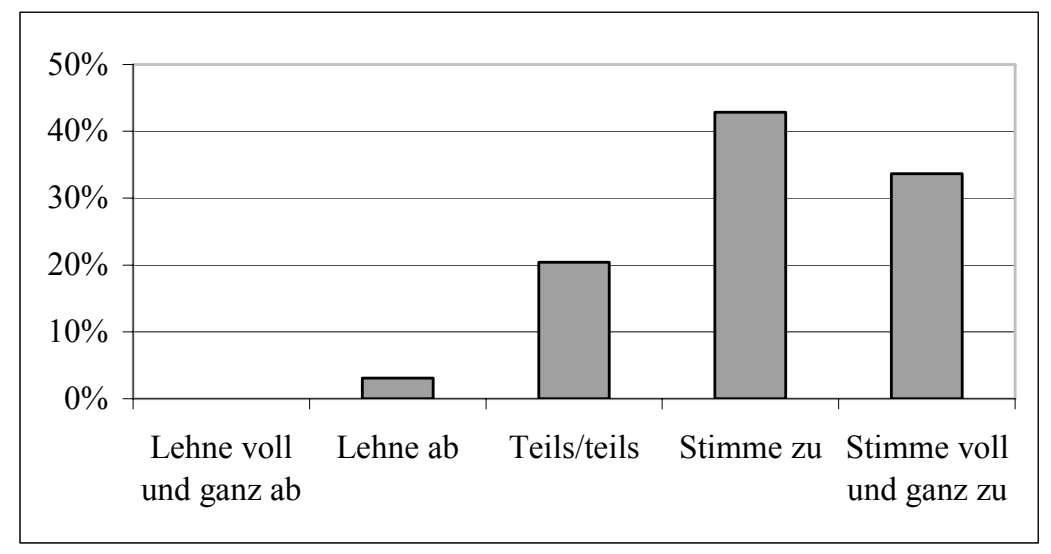

Quelle: Eigene Erhebung, Mitarbeiterbefragung, N=98

Die nächste Abbildung 28 zeigt, ob die befragten Mitarbeiter Fairnessdefizite bei der Entlohnung im Vergleich zu ihren Kollegen im Betrieb empfinden. Die Mitarbeiter, die die Frage beantwortet haben, und die Mitarbeiter, die bereits Erfahrung mit leistungsorientierter Entlohnung gesammelt haben, sind in der Grafik gegenüber gestellt.

ABBILDUNG 28: „ICH FÜHLE MICH BEI DER BEZAHLUNG GEGENÜBER MEINEN KOLLEGEN IM BETRIEB UNGERECHT BEHANDELT“

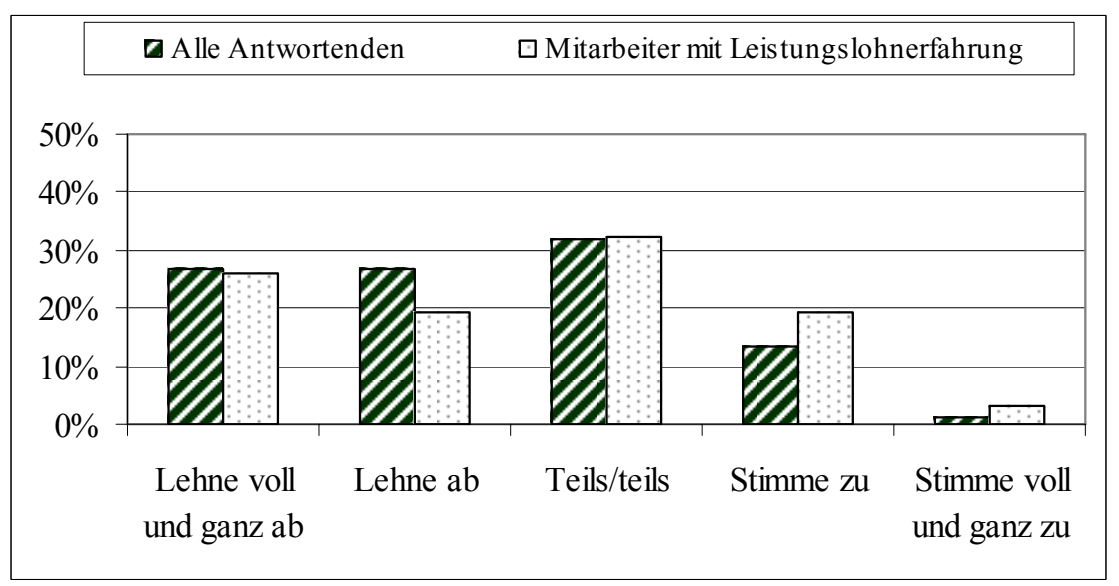

Quelle: Eigene Erhebung, Mitarbeiterbefragung, N=75 bzw. N=31 
Es zeigt sich ein tendenziell heterogenes Bild unabhängig von der Auswahl der Mitarbeitergruppe. Das Statement wird insgesamt von den Mitarbeitern eher abgelehnt, wobei die Mitarbeiter, die bereits über Erfahrungen mit Leistungsentgelten verfügen, dem Statement demnach vergleichsweise mehr Zustimmung geben. Sie empfinden häufiger Ungerechtigkeit gegenüber ihren Kollegen in Bezug auf die Bezahlung.

Die Diskussion der Ergebnisse mit den befragten Mitarbeitern während der Ergebnisworkshops legen jedoch die Vermutung nahe, dass die Frage nach der Erfahrung mit Leistungslöhnen zum Teil falsch aufgefasst wurde, denn viele Mitarbeiter zählen Gratifikationen, die aufgrund eines guten Betriebsergebnisses gewährt werden, zu den Leistungslöhnen. In der Diskussion wurde außerdem deutlich, dass eine Beteiligung der Mitarbeiter am Unternehmenserfolg oder Zulagen, die auf einem guten Betriebsergebnis beruhen, als gerechter und nachvollziehbarer angesehen werden als Prämienlohnsysteme. Dies betrifft vor allem die Mitarbeiter, die bisher im Zeitlohnsystem arbeiten.

Es wurde auch explizit der mögliche Verdrängungseffekt von den Mitarbeitern angesprochen. Die Notwendigkeit, Leistungslohnsysteme einzuführen, sehen die Mitarbeiter nicht, da ihre ohnehin sehr gute Motivation verdrängt werden könnte. An dieser Stelle ist nochmals darauf hinzuweisen, dass es sich bei den befragten Arbeitnehmern nicht um eine repräsentative Stichprobe der in der Landwirtschaft beschäftigten Lohnarbeitskräfte handelt. Die hohe Motivation wird nicht für alle Arbeitnehmer in der Landwirtschaft zutreffen. Außerdem kann sich in dieser Aussage auch die Orientierung am Status Quo widerspiegeln. Da viele der befragten Mitarbeiter im Zeitlohnsystem arbeiten, hängen sie vermutlich am bekannten Vergütungssystem. Während des Workshops wurden auch die von den Betriebsleitern verwendeten Leistungsindikatoren ausführlich diskutiert. Die Mitarbeiter sind der Meinung, dass keiner der vorgestellten Parameter dem Anspruch der Objektivität genüge tut.

Mitarbeiterbefragungen zum Thema leistungsabhängige Vergütung wurden auch vom Personaldienstleister Randstad unter 900 Arbeitnehmern durchgeführt (F.A.Z. 2005: 59). Die Ergebnisse dieser Befragung zeigen, dass $49 \%$ der befragten Mitarbeiter sich keine leistungsorientierte Bezahlung wünschen. $42 \%$ der Befragten würden ihr Verhalten durch ein Leistungslohnsystem nicht ändern. 


\subsection{Zusammenfassung der Ergebnisse hinsichtlich der Arbeitshypothesen}

In diesem Kapitel werden die Ergebnisse der empirischen Analyse in Bezug auf die aufgestellten Hypothesen zusammengefasst. Die Hypothesen eins bis drei widmen sich dem Einfluss des betrieblichen Arbeitsumfelds auf die Wahl des materiellen Anreizsystems und die Hypothesen vier bis sechs dem Mitarbeiter.

\section{Hypothesen zur Einflussgröße „Betriebliches Arbeitsumfeld“}

H1: Leistungsorientierte Entlohnungssysteme spielen in der Tierproduktion eine größere Rolle als im Ackerbau.

Die empirischen Ergebnisse bestätigen, dass Leistungslöhne in der Tierproduktion insgesamt eine größere Rolle als im Ackerbau spielen. Aufgrund der Schwierigkeit, objektive und kardinal messbare Leistungsindikatoren im Ackerbau zu finden, haben Leistungszulagen eine im Verhältnis zum Prämienlohn wichtigere Bedeutung im Ackerbau als in den analysierten Betriebszweigen der Tierproduktion. Die univariaten Auswertungen zur Bedeutung verschiedener Lohnformen in den Betriebszweigen werden durch die logistische Regressionsanalyse gestützt. So wirkt sich eine steigende Bestandesgröße und Bedeutung der Tierproduktion positiv auf die Wahrscheinlichkeit der Erfahrung mit Leistungslöhnen aus, während eine größere Relevanz des Ackerbaus (gemessen an der Ackerfläche) eine negative Wirkung auf die Zielgröße hat.

H2: In den neuen Bundesländern finden leistungsorientierte Entlohnungssysteme häufiger Anwendung als in den alten Bundesländern.

Mit Hilfe der logistischen Regression wurde der Einfluss verschiedener Variablen auf die Erfahrung der Betriebsleiter mit leistungsorientierter Entlohnung ermittelt. Im Vorfeld durchgeführte $\mathrm{Chi}^{2}$-Tests ergaben einen signifikanten Unterschied zwischen dem Betriebssitz und der Erfahrung mit leistungsorientierter Entlohnung. Wenn die Betriebsleiter in den neuen Bundesländern wirtschaften, verfügen sie häufiger über Erfahrung mit Leistungslöhnen. Dieses Ergebnis lässt sich auf verschiedene Zusammenhänge zurückführen. Es lässt sich pauschal nicht schlussfolgern, dass eine Herkunft aus den neuen Bundesländern und damit eine mögliche Tradition in der DDR üblicher Vergütungsformen der Grund für den festgestellten Unterschied ist. Die befragten Betriebsleiter aus den neuen Bundesländern sind eine heterogene Gruppe, die 
sich aus LPG-Nachfolgern, ortsansässigen Wiedereinrichtern und Alteigentümern zusammensetzt. Einen Hinweis auf eine gewisse DDR-Tradition bieten Rechtsformunterschiede in der Häufigkeit verwendeter Entlohnungsformen. Vergleicht man die Gruppe der Einzelunternehmen mit den eingetragenen Genossenschaften, so zeigt sich, dass in der zweiten Gruppe variable Vergütungsformen in den unterschiedlichen Produktionsrichtungen deutlich häufiger eingesetzt werden. Bei der Berechnung des logistischen Regressionsmodells ist es möglich, die wichtigsten Variablen, die somit den größten Beitrag zur Erklärung der abhängigen Variablen liefern, durch einen Algorithmus auswählen zu lassen. Da die uni- und bivariaten Verfahren den Einfluss des Bundeslandes und der Rechtsform auf das Vergütungssystem nahe legten, wurden sie neben verschiedenen anderen Variablen zur Auswahl gestellt. Es ergab sich aber, dass die Anzahl der Mitarbeiter die entscheidende Variable ist, und nicht das Bundesland oder die Rechtsform. Die vorgestellten Beziehungen zwischen den dargestellten unabhängigen und der abhängigen Variablen sind also wohlmöglich Scheinzusammenhänge, die durch die Betriebsgröße erklärt werden.

H3: Je mehr Mitarbeiter beschäftigt sind, desto höher ist die Wahrscheinlichkeit des Einsatzes leistungsorientierter Entgeltsysteme.

Die logistische Regression zeigt den Betriebsgrößeneinfluss auf die Wahrscheinlichkeit des Einsatzes leistungsorientierter Entgeltsysteme. Die Anzahl der beschäftigten Lohnarbeitskräfte ist neben den Betriebsleitereinstellungen zu Anreizsystemen die wichtigste erklärende Variable, wodurch die dritte Hypothese bestätigt wird. Die Bestätigung der Hypothese ist konsistent mit der ökonomischen Theorie, nach der die individuelle Arbeitsleistung mit ansteigender Gruppengröße schwieriger zu messen ist, wodurch moral hazard begünstigt wird. 
Hypothesen zur Einflussgröße „Mitarbeiter“

H4: Die Mitarbeiter in der Landwirtschaft schätzen ihren Arbeitsplatz

Sowohl die Betriebsleiter- als auch die Mitarbeiterbefragung zeigen, dass die Mitarbeiter am Arbeitsplatz Landwirtschaft hängen. Bei der Diskussion dieses Ergebnisses mit den Mitarbeitern wurden mehrere Eigenschaften ihrer Tätigkeit als Grund für die große Arbeitszufriedenheit genannt. Hierzu zählen das Arbeiten in der Natur, die abwechslungsreichen Aufgaben, die durch die klimatischen Rahmenbedingungen beeinflusst werden, das direkte Feedback durch die Arbeit selbst und die weitgehend selbständige Arbeit, deren Takt nicht durch eine Maschine vorgegeben ist. Die langen und nicht planbaren Arbeitszeiten führen nach Ansicht der befragten Mitarbeiter dazu, dass Arbeitskräfte in der Landwirtschaft verbleiben, die sich für die Tätigkeit berufen fühlen. Die Implikationen dieser bestätigten Hypothese für die Gestaltung von Lohnsystemen werden in Kapitel 6 diskutiert.

H5: Die Wertschätzung finanzieller Anreize ist bei den Arbeitnehmern in der Landwirtschaft hoch.

Die Ergebnisse der Befragungen ermöglichen keine eindeutige Bestätigung der Hypothese. Finanzielle Anreize sind für die Mitarbeiter nicht das wichtigste Anreizsystem und rangieren hinter der Sicherheit des Arbeitsplatzes und dem Betriebsklima. Die Mitarbeiter bestätigten in den Ergebnisworkshops diese Reihenfolge, wiesen jedoch explizit auf das niedrige Lohnniveau hin, das nach ihrer Meinung in keinem angemessenen Verhältnis zur Arbeitsbelastung stünde. Aus dieser Aussage lässt sich eine Wertschätzung finanzieller Anreize ableiten.

H6: Die Hierarchieebene der Mitarbeiter hat einen Einfluss auf die Wahl des Anreizsystems.

Leistungsorientierte Vergütungsformen, die auf kardinal messbaren Indikatoren beruhen, werden häufiger in der Produktion verwendet als auf Führungsebene. Dieses Ergebnis verdeutlicht die Schwierigkeit der Leistungsbemessung bei Führungskräften. Wie prognostiziert, spielen Erfolgsbeteiligungen der Führungskräfte in den analysierten Betrieben eine wichtigere Rolle als in der Produktion, da sie einen direkteren Einfluss auf den Unternehmenserfolg als die Mitarbeiter in der Produktion haben. 


\section{$6 \quad$ Handlungsempfehlungen für die landwirtschaftliche Praxis}

In diesem Kapitel werden Handlungsempfehlungen zur Gestaltung von Anreizsystemen und insbesondere zum Einsatz leistungsorientierter Entlohnungssysteme in der Landwirtschaft aus den theoretischen und empirischen Ergebnissen der Arbeit abgeleitet. Wie bereits zu Beginn der Arbeit angeführt, beschränken sich die empirischen Ergebnisse vorrangig auf die Produktionsrichtungen Ackerbau, Schweineproduktion (Mast und Sauenhaltung) und Milchproduktion. Die Untersuchung leistungsorientierter Entlohnungssysteme im Obst-, Gemüse-, Weinbau wird nicht aufgegriffen.

\subsection{Leitfaden zur leistungsorientierten Lohngestaltung}

Die Handlungsempfehlungen für die landwirtschaftliche Praxis orientieren sich an dem in Abbildung 29 dargestellten Leitfaden. Dieser wird zunächst in Kürze vorgestellt. Im Anschluss werden die einzelnen Entscheidungsschritte bei der Implementierung leistungsorientierter Lohnsysteme detaillierter anhand der theoretischen Vorüberlegungen und empirischen Ergebnisse diskutiert.

Bei materiellen Anreizsystemen hat der Betriebsleiter die Wahl zwischen Zeitlohn- und Leistungslohnsystemen. Erfolgs- und Kapitalbeteiligungen sollen aus Vereinfachungsgründen in diesem Leitfaden zum Leistungslohn gezählt werden.

Entscheidet sich der Betriebsleiter für ein Leistungslohnsystem, so ist zu prüfen, ob die Effektivität extrinsischer Anreize gegeben ist. Ist dies nicht der Fall, so sollte der Betriebsleiter auf Zeitlohnsysteme ausweichen. Außerdem muss der Betriebsleiter überlegen, ob eine zielorientierte Steuerung im Betrieb möglich ist (siehe Kapitel 4.2). Ist die zielorientierte Steuerung nicht möglich und aufgrund der betrieblichen Gegebenheiten auch nicht zu verbessern, dann ist ein Zeitlohnsystem anzuwenden.

Sind die Erfolgsbedingungen leistungsorientierter Vergütung erfüllt, so werden im nächsten Schritt die Leistungsindikatoren festgelegt. Tritt nach der Implementierung des leistungsorientierten Entlohnungssystems der gewünschte Erfolg nicht ein, kann der Betriebsleiter auf den Zeitlohn oder immaterielle Anreizsysteme zurückgreifen. 
ABBILDUNG 29: LEITFADEN ZUM EINSATZ LEISTUNGSORIENTIERTER ENTLOHNUNG

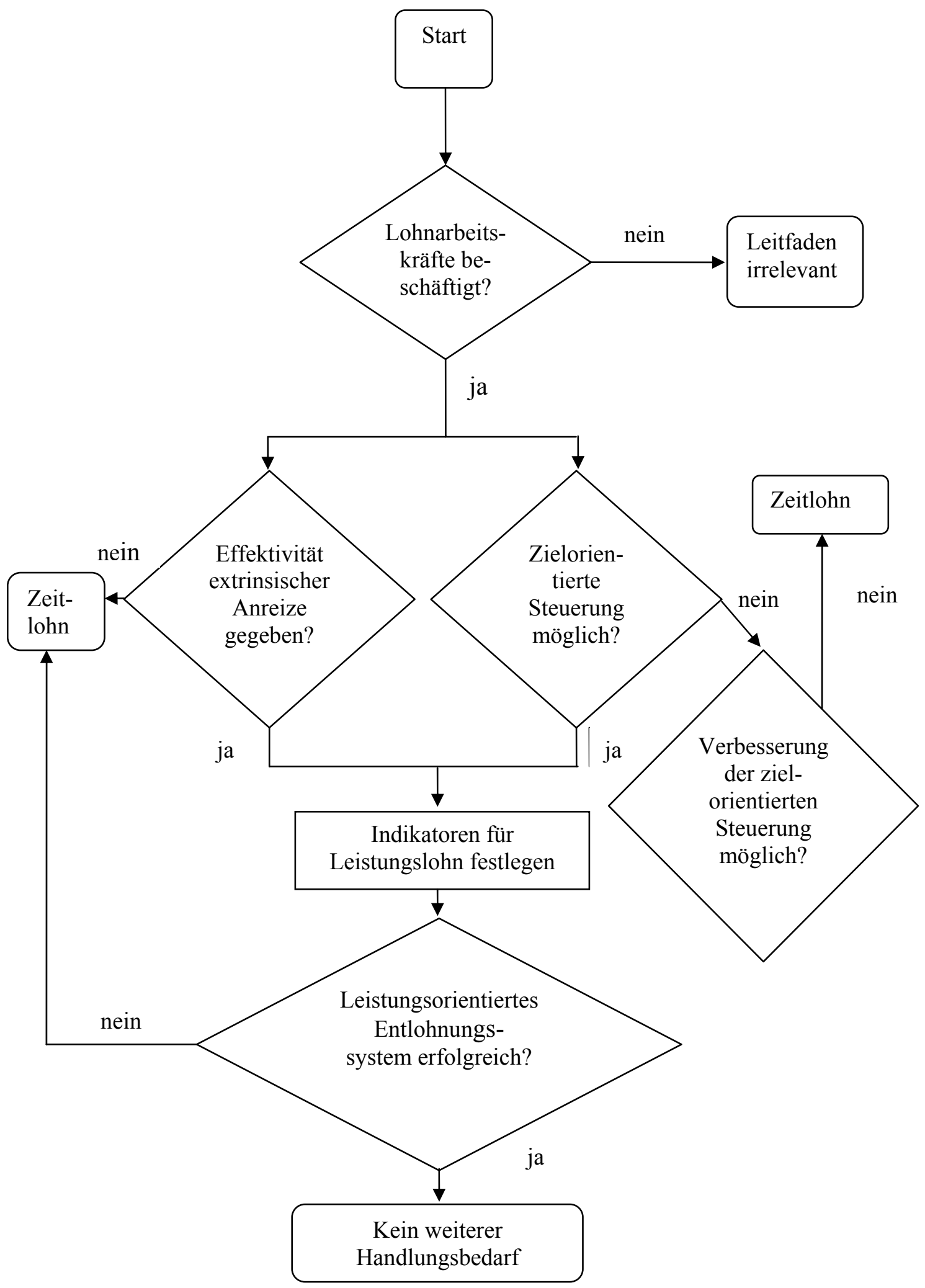

Quelle: DAVIER ET AL. 2006: 50 


\subsubsection{Zur Effektivität extrinsischer Anreize in der Landwirtschaft}

Die Effektivität extrinsischer Anreize ist ein wichtiges Erfolgskriterium für die leistungsorientierte Lohngestaltung und wird von verschiedenen Einflussfaktoren bestimmt. Eine allgemeingültige Handlungsempfehlung, die für alle betrieblichen Situationen gilt, ist deshalb nicht ableitbar. Letztendlich liegt es in der Ermessenssache und der persönlichen Einschätzung einer jeden Führungskraft, diese Faktoren für den eigenen Betrieb abzuschätzen. Gestützt durch die theoretischen und empirischen Ergebnisse kann diese Entscheidung jedoch vereinfacht werden.

Eine leistungsorientierte Entlohnung kann nur der Mitarbeitermotivation zuträglich sein, wenn der betreffende Mitarbeiter finanzielle Anreize auch schätzt. Dies ist eine Konsequenz aus den Valenztheorien der Motivation. Aus der Perspektive der Maslowschen Bedürfnishierarchie ist zunächst sicherzustellen, dass die Mitarbeiter durch die Entlohnung ihren Lebensunterhalt auch decken können, um existentielle Bedürfnisse wie Wohnen und Essen bestreiten zu können. Diese Grundbedürfnisse werden in den Mischformen leistungsorientierter Vergütung wie dem Grundlohn mit Leistungszulage oder Prämie in der Regel durch eine feste Basisvergütung abgegolten. Für Mitglieder der Tarifparteien regeln die Tarifverträge die Grundsicherung durch einen tariflichen Grundlohn.

Aus der Sicht der Motivationstheorie von Herzberg bieten finanzielle Anreize keinen Beitrag zur Motivation der Mitarbeiter, sondern dienen nur als Hygienefaktor, d.h. sie können letztendlich nur Unzufriedenheit mit dem Arbeitsplatz vermeiden. Diese Schlussfolgerung wird durch die empirischen Ergebnisse der Mitarbeiterbefragung gestützt. Es zeigt sich ein signifikant positiver Zusammenhang zwischen der Freude an der Arbeit und dem Arbeitsinhalt, der Würdigung der Arbeitsleistung durch den Chef und der Einbindung in betriebliche Entscheidungen. Hingegen beeinflussen extrinsische Anreize kaum die Motivation der Mitarbeiter.

Dass finanzielle Anreize hinsichtlich ihrer Motivationswirkung bei den befragten Mitarbeitern nicht an erster Stelle stehen, zeigt das Ergebnis der Befragung. Ein sicherer Arbeitsplatz und ein gutes Betriebsklima haben hier eine höhere Bedeutung. Jedoch wurde bei der Diskussion der Ergebnisse mit den Mitarbeitern darauf hingewiesen, dass dies möglicherweise nur bei Mitarbeitern der Fall ist, deren Lohn den Grundbedarf bereits abdeckt. Eine Schlussfolgerung, die auch Maslows Bedürfnishierarchie nahe 
legt, nach der eine Bedürfnisebene nur so lange verhaltensbestimmend ist, bis sie befriedigt ist. Diese Vermutung der Mitarbeiter wird jedoch durch ein Befragungsergebnis der SÄCHSISCHEN LANDESANSTALT FÜR LANDWIRTSCHAFT widerlegt, nach dem Mitarbeiter juristischer Personen, die durchschnittlich geringere Einkommensmöglichkeiten als Einzelunternehmen bieten, gute Verdienstmöglichkeiten erst an vierter Stelle hinsichtlich einer positiven Motivationswirkung nennen (SÄChSISCHE LANDESANSTALT FÜR LANDWIRTSCHAFT 2002: 32). Auch landwirtschaftliche Berater mit Erfahrungen in der Führung von landwirtschaftlichen Großbetrieben empfehlen, finanzielle Anreize nicht über zu bewerten, da sie auf Dauer ein schlechtes Betriebsklima nicht kompensieren könnten (KoESLING 2006: 26).

Neben der Wertschätzung finanzieller Anreize ist es motivationstheoretisch von großer Bedeutung, dass die Mitarbeiter die zum Erhalt der Belohnung notwendige Leistung auch erbringen können. D.h., die Betriebsleiter müssen realistische Zielvorgaben formulieren oder durch Weiterqualifikation der Mitarbeiter dazu beitragen, dass diese die Ziele auch erreichen können. Je nachdem wie die Mitarbeiter ihre persönliche Rolle im Unternehmen und ihre eigenen Fähigkeiten wahrnehmen, fällt ihr Urteil darüber aus, ob sie ein durch die Betriebsleitung gesetztes Ziel auch als erreichbar einschätzen.

Experimentelle Ergebnisse und die theoretischen Ausführungen zur Bedeutung intrinsischer Motivation zeigen, dass extrinsische Anreize unter Umständen intrinsische Motivation verdrängen werden. Die Wechselwirkung von intrinsischer und extrinsischer Motivation kann positiv, neutral oder negativ sein und wird von vielen Faktoren beeinflusst, so dass eine Aussage über den Gesamteffekt leistungsorientierter Entlohnung nur schwer zu treffen ist.

Als einfache Formel kann festgehalten werden, dass intrinsische Motivation nur dort zerstört werden kann, wo sie vorhanden ist. Die Ergebnisse von Betriebsleiter- und Mitarbeiterbefragung und die Diskussionen während der Ergebnisworkshops zeigen aber, dass die Motivation der Mitarbeiter in der Landwirtschaft in der durch die Befragung repräsentierten Stichprobe als sehr hoch angesehen werden muss. Ein Ergebnis, das auch mit dem positiven Empfinden der Tätigkeit in der Landwirtschaft zusammenhängt. Eine abwechslungsreiche und flexible Aufgabe in der Natur macht den Arbeitsplatz für die befragten Mitarbeiter besonders attraktiv und auch die Betriebsleiter, die einen breiten Querschnitt über die Lohnarbeitsbetriebe in den alten 
und neuen Bundesländern widerspiegeln, bestätigen eine hohe Motivation ihrer beschäftigten Lohnarbeitskräfte, was gegen eine leistungsorientierte Entlohnung spricht, da diese hohe intrinsische Motivation verdrängt werden könnte.

Die Wahrscheinlichkeit, dass durch ein leistungsorientiertes Entlohnungssystem intrinsische Motivation verdrängt wird, steigt, wenn die Mitarbeiter das Vergütungssystem als Fremdkontrolle und Mangel an Anerkennung interpretieren. Die befragten Betriebsleiter, die bereits Erfahrung mit dieser Vergütungsform gesammelt haben, unterscheiden sich hinsichtlich dieser möglichen Risiken und lassen sich nach ihren unterschiedlichen Erfahrungen mit leistungsorientierter Entlohnung klassifizieren. Eine Gruppe von Betriebsleitern, die im Vergleich zu den beiden anderen Gruppen durch kleinere Betriebe mit einem höheren Eigentumsanteil und weniger Mitarbeitern charakterisiert ist, ist eher kritisch hinsichtlich der möglichen Verhaltenswirkungen leistungsorientierter Entlohnung. Diese Gruppe von Betriebsleitern hat ihren Betriebssitz häufig in den alten Bundesländern und betreibt vielfach ökologischen Landbau.

Eine besondere Rolle spielt außerdem Fairness für die Motivation der Mitarbeiter. Fühlen sich die Mitarbeiter durch das Entlohnungssystem ungerecht behandelt, so ist eine positive Motivationswirkung in Frage gestellt. Menschen orientieren sich in ihrem Urteil hinsichtlich der Lohngerechtigkeit an Referenzpersonen im und außerhalb des Betriebes, von denen sie denken, dass sie ähnliche Fähigkeiten einbringen.

Die befragten Mitarbeiter fühlen sich im Mittel gerecht behandelt gegenüber ihren Kollegen im Betrieb und im Vergleich zu anderen Betrieben. Es handelt sich jedoch vor allem um Mitarbeiter, die im Zeitlohnsystem arbeiten. Ein kritischer Punkt ist hier vor allem die Bemessungsgrundlage der Leistungslöhne. In der Diskussion mit den Mitarbeitern über die von den befragten Betriebsleitern eingesetzten Leistungsindikatoren wurde deutlich, dass faktisch alle Indikatoren als zu störanfällig gegenüber Einflüssen wie der Witterung oder der Leistung der Kollegen eingeschätzt wurden, um eine gerechte Basis für eine leistungsorientierte Entlohnung darzustellen.

Die Wahrnehmung von Gerechtigkeit kann durch die Betriebsleiter verbessert werden, indem klargemacht wird, wie Unterschiede in der Entlohnung zustande kommen. Empirische Ergebnisse zeigen außerdem, dass sich Menschen in ihrer Bewertung von Situationen am Status Quo orientieren. Führt man ein anderes Lohnsystem ein, so wird 
die neue Situation häufig mit der alten Situation, an die man gewöhnt ist, verglichen. Da man einmal lieb gewonnenes ungern verliert, neigen viele Menschen zur Verlustaversion. Neue Systeme lassen sich folglich mit neuen Mitarbeitern leichter einführen als mit alten. Da jedoch ein Betrieb nur bei Neugründungen oder bei einem Betriebsleiterwechsel in dieser günstigen Situation ist, gilt es in allen anderen Betrieben, den Menschen zu verdeutlichen, dass die alte Situation nicht mehr zur Disposition steht. Außerdem folgt aus der Orientierung am Status Quo und der Verlustaversion, dass es schwierig ist, bei Einführung eines Leistungslohnsystems einen Teil des bisherigen Lohnes als variabel zu erklären. Betriebsleitern ist $\mathrm{zu}$ empfehlen, den bisherigen Lohn als Ausgangspunkt zu nehmen und den variablen Anteil bei Erreichen bestimmter Leistungen zusätzlich zu gewähren. Wie die Ergebnisdiskussion zeigte, ist die erste Variante für die befragten Mitarbeiter völlig unakzeptabel und würde zur Demotivation führen. Diese Feststellung deckt sich auch mit den Erfahrungen der SÄCHSISCHEN LANDESANSTALT FÜR LANDWIRTSCHAFT (2002: 40).

\subsubsection{Möglichkeiten der zielorientierten Steuerung in den Betrieben}

Kann ein Betriebsleiter die Effektivität extrinsischer Anreize für seine Mitarbeiter bestätigen, so ist zu klären, ob auch die Voraussetzungen für eine zielorientierte Steuerung gegeben sind.

Ein wichtiges Element der zielorientierten Steuerung ist die Klarheit über die Unternehmensziele. Die empirische Analyse hat gezeigt, dass sich die Einschätzung der Betriebsleiter und Mitarbeiter in Bezug auf die Zielklarheit unterscheidet. Die Mitarbeiter sind überwiegend der Meinung, dass sie sich über die Ziele des Unternehmens klar sind, während die befragten Betriebsleiter diese Aussage für ihre Mitarbeiter nicht unbedingt teilen. Dies verdeutlicht die Notwendigkeit, mit den Mitarbeitern über die angestrebten Ziele zu sprechen.

Sind die Oberziele der Unternehmensleitung definiert und an die Mitarbeiter im Rahmen von regelmäßigen Gesprächen weiter getragen, so gilt es für die erfolgreiche Einführung leistungsorientierter Vergütungssysteme, die Ziele auf der jeweiligen Hierarchieebene des Betriebes zu definieren. Ein Vorteil vieler landwirtschaftlicher Betriebe sind einfache Organisationsformen mit flachen Hierarchien. Wenn beispielsweise als Oberziel im Unternehmen die Gewinnmaximierung festgelegt wird, so gilt es, Kennziffern auf Ebene der Produktion zu finden, die mit der Erreichung des 
Oberziels kompatibel sind. In der Erfüllung der Erfolgsbedingung der Zielkompatibilität besteht in landwirtschaftlichen Betrieben eine große Herausforderung, wie die Diskussion möglicher Leistungsparameter in den unterschiedlichen Betriebszweigen zeigen wird. Denn eine Erhöhung von Produktivitätskennzahlen, wie zum Beispiel der Hektarerträge, geht häufig mit einer erhöhten Intensivierung der Produktion einher. Dies kann dazu führen, dass durch ein Leistungslohnsystem, das sich allein am Outputniveau orientiert, Fehlanreize gesetzt werden, die dazu führen, dass das Oberziel des Unternehmens nicht erreicht wird. Die Praxis zeigt, dass Betriebsleiter versuchen, solchen Fehlanreizen durch die Kopplung des Leistungsindikators an weitere Ziele und Bedingungen entgegen zu wirken. Die Festlegung mehrerer Ziele ist jedoch im Hinblick auf den potentiellen Erfolg des Leistungslohnsystems kontraproduktiv, da mit steigender Zielanzahl die Abschätzung der Zielwirkungen der verschiedenen Parameter schwieriger wird. Es wird daher empfohlen, maximal vier bis fünf Leistungsparameter festzulegen.

\subsection{Indikatoren für Leistungslöhne in der Landwirtschaft}

Im folgenden Abschnitt werden mögliche Leistungsindikatoren für verschiedene landwirtschaftliche Betriebszweige vorgestellt. Für jeden Betriebszweig werden die Leistungskriterien entlang des Produktionsprozesses und auf der Aufwands- und Ertragsseite vorgestellt. Für die Tierproduktion werden Leistungsindikatoren am Beispiel der unterschiedlichen Verfahrensschritte oder, dort wo es sinnvoller erscheint, anhand von produktionstechnischen Kennzahlen aufgezeigt. Zusammengefasst aus den vorherigen Kapiteln sollten die Leistungskriterien die folgenden Anforderungen erfüllen. Sie müssen

- objektiv messbar,

- dem Mitarbeiter eindeutig zuzuordnen,

- einfach,

- kostenrelevant,

- und mit wenig Kontrollaufwand verbunden sein. 
Die Besonderheiten der landwirtschaftlichen Produktion führen dazu, dass es faktisch unmöglich ist, diese Erfolgsbedingungen alle zu erfüllen. Es werden deshalb die betrieblichen Voraussetzungen aufgeführt, die eine Annäherung an die oben genannten Erfolgsbedingungen ermöglichen. Jeder Betriebsleiter möge vorab prüfen, ob diese Bedingungen auch gegeben sind und die Implementierung eines Leistungslohnsystems wirklich notwendig ist.

\subsubsection{Leistungsindikatoren für den Ackerbau}

Die Tabelle 31 zeigt mögliche Leistungsindikatoren im Ackerbau entlang des Produktionsprozesses auf. Produktionsverfahren im Ackerbau beginnen mit der Aussaat und enden in der Regel mit der Bodenbearbeitung. Es ist zu diskutieren, ob die Bodenbearbeitung am Anfang des Produktionsprozesses oder am Ende steht. Aufgrund der zunehmenden Bedeutung von Direktsaatverfahren wird sie im zeitlichen Ablauf als letzter Verfahrensschritt aufgeführt, da die Bodenbearbeitung gegebenenfalls auch entfallen kann. Die Tabelle ist wie folgt gegliedert. Für jeden Arbeitsschritt ist ein Indikator aufgeführt, der als Basis für ein Leistungslohnsystem verwendet werden könnte. Es werden dann die für die Verwendung des Indikators notwendigen Kontrollen, betrieblichen Voraussetzungen und potentiellen Fehlanreize aufgeführt. Eine Einschätzung der zur Kontrolle der Leistungsindikatoren notwendigen Transaktionskosten und die Störanfälligkeit der Arbeitsqualität erfolgt im Anschluss. Die Leistungsindikatoren werden dann nach ihrer Vorzüglichkeit rangiert. 
TABELLE 31: LEISTUNGSINDIKATOREN FÜR DEN ACKERBAU NACH ARBEITSSCHRITTEN

\begin{tabular}{|c|c|c|c|c|c|c|c|}
\hline \multirow{2}{*}{$\begin{array}{l}\text { Arbeits- } \\
\text { schritt }\end{array}$} & \multirow[t]{2}{*}{ Indikator } & \multirow[t]{2}{*}{ Notwendige Kontrollen } & \multirow{2}{*}{$\begin{array}{c}\text { Betriebliche } \\
\text { Voraussetzungen }\end{array}$} & \multirow{2}{*}{$\begin{array}{l}\text { Mögliche Fehlanreize } \\
\text { (exemplarisch) }\end{array}$} & \multicolumn{2}{|c|}{ Bewertung von } & \multirow{2}{*}{$\begin{array}{l}\text { Vorzüglich- } \\
\quad \text { keit }\end{array}$} \\
\hline & & & & & $\begin{array}{l}\text { Höhe der } \\
\text { Transaktions- } \\
\text { kosten }\end{array}$ & $\begin{array}{l}\text { Störanfällig- } \\
\text { keit der } \\
\text { Arbeits- } \\
\text { qualität }\end{array}$ & \\
\hline Aussaat & $\begin{array}{l}\text { ha/Zeiteinheit bei } \\
\text { vorgesehener Qualität }\end{array}$ & $\begin{array}{l}\text { Saattiefe, } \\
\text { Fahrgeschwindigkeit, } \\
\text { ausgebrachte } \\
\text { Saatmenge/ha }\end{array}$ & $\begin{array}{l}\text { Erfassung der Fahr- } \\
\text { geschwindigkeit, der } \\
\text { geleisteten ha/Zeiteinheit } \\
\text { pro Fahrer }\end{array}$ & $\begin{array}{l}\text { Falsche Fahrgeschwindigkeit, } \\
\text { Saattiefe, verstopfte Särohre, } \\
\text { falsche Maschineneinstellung }\end{array}$ & $\uparrow$ & $\uparrow$ & 6 \\
\hline Düngung & $\begin{array}{l}\text { Ausgebrachte } \\
\text { Düngermenge bei } \\
\text { mittlerer Hof-Feld- } \\
\text { Entfernung }\end{array}$ & $\begin{array}{l}\text { Kontrolle der vor- } \\
\text { gesehenen Dünger- } \\
\text { mengen/Schlag, } \\
\text { Fahrgeschwindigkeit, } \\
\text { Einstellung des } \\
\text { Düngerstreuers } \\
\end{array}$ & $\begin{array}{l}\text { Erfassung der Fahr- } \\
\text { geschwindigkeit, der } \\
\text { geleisteten ha/Zeiteinheit } \\
\text { pro Fahrer }\end{array}$ & $\begin{array}{l}\text { Überlappungen am } \\
\text { Vorgewende, ungenaue } \\
\text { Verteilung der Menge auf dem } \\
\text { Schlag }\end{array}$ & $\rightarrow$ & $\downarrow$ & 4 \\
\hline $\begin{array}{l}\text { Pflanzen- } \\
\text { schutz }\end{array}$ & $\begin{array}{l}\text { Ausgebrachte } \\
\text { Pflanzenschutzmengen } \\
\text { bei mittlerer Hof-Feld- } \\
\text { Entfernung pro } \\
\text { Zeiteinheit }\end{array}$ & $\begin{array}{l}\text { Kontrolle des } \\
\text { vorgesehenen } \\
\text { Düsendrucks, Wasser- } \\
\text { menge, Mitteleinsatz, } \\
\text { Fahrgeschwindigkeit, } \\
\text { Wetter }\end{array}$ & $\begin{array}{l}\text { Erfassung der Fahr- } \\
\text { geschwindigkeit, der } \\
\text { geleisteten ha/Zeiteinheit } \\
\text { pro Fahrer }\end{array}$ & $\begin{array}{l}\text { Vernachlässigung der } \\
\text { korrekten Befüllung und } \\
\text { Dosierung, schnelles Fahren } \\
\text { auf der Straße }\end{array}$ & $\uparrow$ & $\uparrow$ & 5 \\
\hline Ernte & Tonnage $/ \mathrm{h}$ & $\begin{array}{l}\text { Messung der } \\
\text { Druschleistung und } \\
\text {-qualität }\end{array}$ & $\begin{array}{l}\text { Automatische } \\
\text { Mengenerfassung, Daten } \\
\text { über erzielbare } \mathrm{t} / \mathrm{h} \text { in } \\
\text { Abhängigkeit vom } \\
\text { Ertragsniveau und den } \\
\text { Erntebedingungen }\end{array}$ & $\begin{array}{l}\text { Zu hohe Schnitthöhe, } \\
\text { Schneidwerkverluste, } \\
\text { Überlastung der Maschine, } \\
\text { Vernachlässigung der } \\
\text { Druschqualität (Bruchkorn) }\end{array}$ & $\downarrow$ & $\rightarrow$ & 1 \\
\hline Transport & Kopplung an Tonnage $/ \mathrm{h}$ & $\begin{array}{l}\text { Zuordnung der } \\
\text { transportierten Tonnage }\end{array}$ & Wiegen der Anhänger & $\begin{array}{l}\text { Schnelles Fahren (höherer } \\
\text { Verschleiß), Überladung }\end{array}$ & $\downarrow$ & $\rightarrow$ & 2 \\
\hline $\begin{array}{l}\text { Boden- } \\
\text { bearbeitung }\end{array}$ & $\begin{array}{l}\text { ha/Zeiteinheit bei } \\
\text { mittlerer Hof-Feld- } \\
\text { Entfernung und } \\
\text { vorgegebener } \\
\text { Bearbeitungstiefe }\end{array}$ & $\begin{array}{l}\text { Kontrolle von } \\
\text { Bearbeitungstiefe, } \\
\text { Fahrgeschwindigkeit }\end{array}$ & $\begin{array}{l}\text { Erfassung der } \\
\text { Fahrgeschwindigkeit, der } \\
\text { geleisteten ha/Zeiteinheit } \\
\text { pro Fahrer }\end{array}$ & $\begin{array}{l}\text { Zu geringe Bearbeitungstiefe, } \\
\text { Unterlassung von } \\
\text { Maschinenwartung und } \\
\text {-nacheinstellung }\end{array}$ & $\downarrow$ & $\rightarrow$ & 3 \\
\hline
\end{tabular}

Quelle: Eigene Darstellung 
Im Produktionsschritt der Aussaat wäre es in der Praxis denkbar, eine Prämie für die erreichten „ha/Zeiteinheit““ zu gewähren. Diese Entlohnung würde sogar einem Zeitakkord entsprechen, wenn man den Mitarbeiter nur nach der Menge der geleisteten Hektar im Abrechnungszeitraum (Woche oder Monat) entlohnen würde.

Dieses System wurde in der ehemaligen DDR in den landwirtschaftlichen Produktionsgenossenschaften angewendet. Dort gab es umfangreiche Normenkataloge, mit Richtwerten zur Bestimmung der Normleistung bei der Arbeit mit einer bestimmten Maschinenausstattung (GABLER 1995: 159 ff.). Von diesem System wurde aufgrund der Notwendigkeit der ständigen Normanpassung und der Berücksichtigung unterschiedlicher Bodenverhältnisse in einigen Betrieben nach der Wende wieder Abstand genommen (TANNEBERGER 1997: 18). Schon in der DDR-Zeit war diese Form der Entlohnung in der Pflanzenproduktion wegen der oben aufgeführten Schwierigkeiten auch bei den Arbeitgebern nicht sehr beliebt (GABLER 1995: 160).

Da bei der Aussaat jedoch nicht nur die Mengenleistung von Bedeutung ist, sondern die Qualität der Aussaat einen starken Einfluss auf den Ertrag hat, ist es dringend notwendig, auch die vorgesehene Qualität zu bestimmen. Hierzu gehört die Festlegung der Fahrgeschwindigkeit, die bei mechanischen Standarddrillmaschinen einer Maximalgeschwindigkeit entsprechen würde. Bei Mulch- oder Direktsaatdrillmaschinen ist hingegen häufig auch eine Minimalgeschwindigkeit für die Saatqualität von Bedeutung. Gibt man die Fahrgeschwindigkeit vor und berücksichtigt Wende-, Weg-, Befüll- und Kontrollzeiten, so ist der Spielraum des Mitarbeiters sehr eng. Der Parameter ist außerdem störanfällig gegenüber Flüchtigkeitsfehlern. Auch ist es möglich, dass die Mitarbeiter auf Kontrollen an der Drillmaschine oder eine zwischenzeitliche Anpassung der Maschineneinstellung an die Bodengegebenheiten verzichten, um die Hektarleistung zu steigern. Der Einsatz dieses Parameters ist unter den angeführten Schwierigkeiten überhaupt nur möglich, wenn durch einen Bordcomputer wichtige Parameter wie die Erfassung der tatsächlichen Fahrgeschwindigkeit, die geleisteten ha pro Zeiteinheit und die Ausbringung der geplanten Saatmenge pro ha automatisch festgehalten werden. Anderenfalls müssten diese entscheidenden Daten vom Betriebleiter zusätzlich ermittelt werden. Dies würde zu hohen Transaktionskosten führen, die die Anwendung eines Leistungslohnsystems in diesem Fall nicht sinnvoll erscheinen lassen. Auch beim Einsatz einer modernen Datenerfassung kommt der Betriebsleiter hingegen nicht umhin, die Aussaatqualität 
anhand einer Feldbegehung zu prüfen. Dabei wird jedoch noch nicht deutlich, ob ein Särohr nicht einmal verschlossen war, denn der Betriebsleiter wird kaum jede Reihe prüfen können. Ähnliche Prämien für die geleistete Arbeit pro Zeiteinheit wären für Bodenbearbeitung, Düngung und Pflanzenschutz denkbar, sofern die in Tabelle 31 aufgeführten Kontrollen und Voraussetzungen gegeben sind.

Die Datenerfassung über moderne Bordcomputer ermöglicht die Dokumentation sämtlicher Tätigkeiten auf dem Schlag und verringert damit den Kontrollaufwand für die oben genannten Kriterien. Die schlagbezogene Datenerfassung registriert auch Unterbrechungen sowie Fahrten zwischen den einzelnen Schlägen und Transporte. Schlagbezogene Daten wie die ausgebrachte Menge (z.B. ausgebrachte Düngermenge pro ha) und die benötigte Zeit werden ebenfalls erfasst (AGROCOM 2006). Diese technischen Möglichkeiten bieten dem Betriebsleiter deutliche Erleichterungen bei der Kontrolle der oben genannten Arbeitsschritte. So kann zum Beispiel festgestellt werden, ob der Fahrer während der Ausbringung zwischenzeitlich abgestiegen ist, was bei der Kontrolle der Saattiefe wünschenswert ist. $\mathrm{Ob}$ er dabei tatsächlich Kontrollen durchgeführt hat, ist hingegen nicht überprüfbar. Praxiserfahrungen zeigen, dass die Dokumentation sämtlicher Arbeitsvorgänge zwar betriebswirtschaftliche Analysen erleichtern, jedoch ein hoher Datenerfassungsaufwand und eine permanente Pflege des Datenbestandes notwendig sind (KÜBLER 2007). Auch wenn der technische Fortschritt bei modernstem EDV-Einsatz in der Landwirtschaft die Kontrollen verringert, verbleibt für Aussaat und Pflanzenschutz immer noch die Notwendigkeit der Vorortkontrolle der tatsächlichen Arbeitsqualität. Bei der Düngung ist der Kontrollaufwand hingegen geringer, da vom Bordcomputer die wesentlichen Kennziffern, nämlich die „ausgebrachte Menge pro ha“ mit der hierfür benötigten Zeit, automatisch geliefert werden. Überschneidungen, z.B. am Vorgewende, können die Aussagefähigkeit des Parameters jedoch verzerren. Es ist außerdem fraglich, wie sinnvoll ein solches Kriterium ist, wenn es wesentlich durch die Maschineneinstellung vorgegeben ist und sich der Handlungsspielraum des Fahrers im Wesentlichen auf die Schnelligkeit bei der Befüllung des Düngerstreuers und der Dauer des Übersetzens zwischen verschiedenen Schlägen beschränkt.

In Bezug auf die Störanfälligkeit der beschriebenen Kriterien lässt sich festhalten, dass Aussaat und Pflanzenschutz wesentlich störanfälliger hinsichtlich der Arbeitsqualität sind als die Düngerausbringung. Die Saatqualität kann durch das Leistungskriterium 
negativ beeinflusst werden und es kann letztendlich nur im Nachhinein festgestellt werden kann, wie gut die Arbeitsqualität war. Die Nachkontrolle hat nur eine beschränkte Aussagefähigkeit hinsichtlich der Arbeitsqualität, da der Feldaufgang auch noch von weiteren Faktoren wie Temperatur und Niederschlag nach der Saat bestimmt werden kann. Im Bereich der Pflanzenschutzausbringung ist nicht festzustellen, ob für jede Spritze die Spritzbrühe korrekt angesetzt wurde, da dieser Parameter nicht automatisch erfasst wird. Für die Qualität der Arbeit ist dies jedoch ein entscheidendes Kriterium, da auch Flüchtigkeitsfehler starke negative Auswirkungen auf weitere produktionstechnische Schritte und vor allem die Wirtschaftlichkeit haben können.

Ein mögliches objektiv messbares Kriterium bei der Ernte ist die erzielte „Druschmenge pro Druschstunde in $\mathrm{t} / \mathrm{h}$ “. Da die zu erzielende Tonnage/h neben der Kapazität der Maschinen von der Feuchtigkeit des Druschgutes, dem Ertrag und weiteren Erntebedingungen wie Lager oder Strohreife abhängt, bedarf es einer ausgefeilten Datenbasis für die Berechnung einer Normleistung. Da häufig der Abtransport die Druschleistung pro Stunde auch beeinflusst, könnte die Bezahlung der Abfahrer an die Tonnage/h gebunden werden. In einem Praxisbeispiel, das in einer landwirtschaftlichen Fachzeitschrift aufgeführt ist, schlägt ein Betriebsleiter vor, den Lohn der Abfahrer an die von den Mähdreschfahrern gedroschene Menge zu binden. Die Mähdrescherfahrer erhalten einen leistungsabhängigen Stundenlohn, der sich an der geernteten Tonnage pro Stunde orientiert, und die Abfahrer erhalten $80 \%$ des erzielten Druschlohns. Dadurch werden sie angehalten, Standzeiten zu vermeiden (TANNEBERGER 1997: 18).

Der Kontrollaufwand durch den Betriebsleiter und die dadurch resultierende Transaktionskostenbelastung sind bei der Ausstattung der Mähdrescher mit modernen Bordcomputern vergleichsweise gering. Die Störanfälligkeit des Parameters bewegt sich im Vergleich zu den oben genannten Leistungsindikatoren im mittleren Bereich. Die Aussagefähigkeit des Leistungsparameters hängt jedoch stark von den zur Einordnung notwendigen Betriebsdaten ab, die eine objektive Einschätzung erst ermöglichen.

Erfahrungen mit leistungsabhängiger Entlohnung bei der Bodenbearbeitung in Russland, die auf rein quantitativen Maßstäben wie den bearbeiteten Hektar pro Tag beruhen, zeigen, dass es unabdingbar ist, Qualitätsmerkmale wie die Bearbeitungstiefe in das Vergütungsschema mit aufzunehmen. Um eine hohe Anzahl bearbeiteter Fläche $\mathrm{zu}$ erreichen, vernachlässigen nach der Erfahrung von KOLWALCZYK UND 
SCHMIDT (2006: f.) die Mitarbeiter die richtige Arbeitstiefe, eine gute Einebnung und Stroheinarbeitung. Die Übertragbarkeit von Erfahrungen mit bestimmten Leistungsindikatoren aus anderen Ländern ist jedoch nur bedingt aussagefähig, da soziokulturelle Unterschiede, die sich im Arbeitsverhalten niederschlagen können, berücksichtigt werden müssen.

Leistungsindikatoren anhand von Kosten- und Leistungspositionen des Betriebszweigs Ackerbau, die der Mitarbeiter durch seinen individuellen Leistungsbeitrag beeinflussen kann, sind in der nachfolgenden Tabelle 32 aufgeführt.

TABELLE 32: LEISTUNGSINDIKATOREN FÜR DEN ACKERBAU ANHAND VON KOSTENLEISTUNGSPOSITIONEN

\begin{tabular}{|c|c|c|c|c|c|}
\hline \multirow{2}{*}{$\begin{array}{l}\text { Kosten-/ } \\
\text { Leistungs- } \\
\text { position }\end{array}$} & \multirow[t]{2}{*}{ Indikator } & \multirow{2}{*}{$\begin{array}{l}\text { Notwendige } \\
\text { Kontrollen }\end{array}$} & \multirow{2}{*}{$\begin{array}{l}\text { Betriebliche } \\
\text { Voraussetzungen }\end{array}$} & \multicolumn{2}{|c|}{ Bewertung von } \\
\hline & & & & $\begin{array}{c}\text { Höhe der } \\
\text { Transaktions- } \\
\text { kosten }\end{array}$ & $\begin{array}{l}\text { Störanfällig- } \\
\text { keit des } \\
\text { Leistungs- } \\
\text { indikators }\end{array}$ \\
\hline $\begin{array}{l}\text { Markt- } \\
\text { leistung }\end{array}$ & $\begin{array}{l}\text { Entwicklung der } \\
\text { Hektarerträge } \\
\text { gegenüber einem } \\
\text { Normwert }\end{array}$ & $\begin{array}{l}\text { Individueller } \\
\text { Leistungsbeitrag }\end{array}$ & $\begin{array}{l}\text { Horizontaler und } \\
\text { vertikaler Betriebs- } \\
\text { vergleich }\end{array}$ & $\downarrow$ & $\uparrow$ \\
\hline $\begin{array}{l}\text { Maschinen- } \\
\text { unterhaltung }\end{array}$ & $\begin{array}{l}\text { Unterschreitung } \\
\text { der durch- } \\
\text { schnittlichen } \\
\text { Maschinen- } \\
\text { unterhaltungs- } \\
\text { kosten }\end{array}$ & $\begin{array}{l}\text { Korrektur um nicht } \\
\text { zurechen- } \\
\text { bare Schadens- } \\
\text { fälle }\end{array}$ & $\begin{array}{l}\text { Zuordnung der } \\
\text { Maschinen zu } \\
\text { einem } \\
\text { Schlepperfahrer, } \\
\text { Verfügbare Daten } \\
\text { aus BV }\end{array}$ & $\rightarrow$ & $\uparrow$ \\
\hline Treibstoffe & $\begin{array}{l}\text { Unterschreitung } \\
\text { des durch- } \\
\text { schnittlichen } \\
\text { Treibstoff- } \\
\text { verbrauchs in } \\
\text { Litern }\end{array}$ & $\begin{array}{l}\text { Korrekturab- } \\
\text { schläge in nassen } \\
\text { Jahren }\end{array}$ & $\begin{array}{l}\text { Zuordnung der } \\
\text { Maschinen zu } \\
\text { einem } \\
\text { Schlepperfahrer, } \\
\text { verfügbare Daten } \\
\text { aus BV }\end{array}$ & $\rightarrow$ & $\downarrow$ \\
\hline
\end{tabular}

Quelle: Eigene Darstellung

Die Marktleistung setzt sich zusammen aus der Menge marktfähiger Ware und dem Produktpreis. Es empfiehlt sich nicht, die Mitarbeiter an den Preisschwankungen teilhaben zu lassen, da die Mitarbeiter in der Produktion den Produktpreis kaum beeinflussen können. Angestellte Betriebsleiter haben in höherem Maße Einfluss auf die erzielten Marktpreise, jedoch ist auch hier der Einfluss der Entwicklung an den Agrarmärkten bedeutender als der Betriebsleitereinfluss. Es ist aber möglich, die Entlohnung der Mitarbeiter an die Entwicklung der Hektarerträge in Relation zu einem zuvor festgelegten Normwert zu knüpfen, der sich auf Daten stützt, die aus horizontalen und vertikalen Betriebsvergleichen bezogen werden können. Die Transaktionskosten 
zur Bemessung dieses Leistungsindikators sind gering, der individuelle Leistungsbeitrag der einzelnen Mitarbeiter zur Erzielung hoher Hektarerträge hängt jedoch stark von der Betriebsorganisation $\mathrm{ab}$. Der Leistungsindikator ist sehr störanfällig gegenüber Witterungs- und Standorteinflüssen.

Außerdem könnten Mitarbeiter Ersparnisprämien bei der Maschinenunterhaltung und dem Treibstoffverbrauch erhalten. Diese Ersparnisprämien werden in der Praxis zum Teil auch angewendet, wie die empirischen Ergebnisse zeigen (vgl. Kapitel 5.4.2). Bei Unterschreitung der durchschnittlichen Maschinenunterhaltungskosten können die Mitarbeiter eine Prämie erhalten. Diese kann auch als Gruppenprämie gewährt werden, was den Vorteil hat, dass die Mitarbeiter gegenseitig darauf achten, ob auch sorgsam mit den Maschinen umgegangen wird. Der Nachteil ist, dass Trittbrettfahrereffekte auftreten können. Wird die Prämie als Einzelprämie gewährt, so müssen verschiedene betriebliche Voraussetzungen gegeben sein. Zum einen ist es notwendig, dass jedem Mitarbeiter eine eigene Maschine zugeordnet wird. Zum zweiten müssen in diesem Fall Vergleichswerte für die jeweilige Maschine vorliegen. Darüber hinaus muss der Maschinenbestand immer ein konstantes Durchschnittsalter haben, da die Maschinenunterhaltungskosten mit dem Alter der Maschinen ansteigen und Vergangenheitswerte dann nicht als Vergleichsbasis herangezogen werden können.

Eine weitere wichtige Kostenposition im Ackerbau, die vom Fahrer deutlich durch sein Fahrverhalten beeinflusst werden kann, ist der Kraftstoffverbrauch. Nach MANN (2006: 30) lassen sich bei guter Schulung der Mitarbeiter und optimalem Maschinensatz $15 \%$ der Kraftstoffkosten sparen, ohne Leistung einzubüßen. Die Transaktionskosten zur Kontrolle des Treibstoffverbrauchs sind, im Vergleich zu den zuvor genannten Indikatoren, in Abhängigkeit von den betrieblichen Gegebenheiten als gering bis mittelhoch einzuschätzen. Der Kontrollaufwand ist gering, wenn für jedes Jahr und jede Zug- bzw. Erntemaschine der Treibstoffverbrauch insgesamt und die geleisteten Betriebsstunden bereits erfasst werden. Höher ist er hingegen, wenn diese Erfassung aufgrund des Leistungslohnsystems erfolgen muss. Störanfällig ist der Indikator bei schwierigen Bodenverhältnissen in einem nassen Jahr und wenn sich der Einsatzbereich der Zugmaschine im Laufe der Jahre verändert. Da der Treibstoffverbrauch von den Mitarbeiteren bei der Bodenbearbeitung auch durch eine geringere Bearbeitungstiefe reduziert werden kann (DLG 2007: 6 f.), liegt hier eine potentielle Quelle für 
Fehlanreize vor. Die Bearbeitungstiefe müsste vom Betriebsleiter ggf. zumindest stichprobenartig kontrolliert werden.

Die Ausführungen $\mathrm{zu}$ den in Tabelle 31 aufgeführten Leistungsindikatoren verdeutlichen, dass die Akkordfähigkeit (vgl. Kapitel 4.2.3) der Produktionsverfahren im Ackerbau in der Regel nicht gegeben ist. Dies erklärt auch die geringe Bedeutung von Leistungslöhnen im Vergleich zu Zeitlöhnen in den untersuchten Betrieben (vgl. Kapitel 5.4.1). Die Transaktionskosten für die Kontrolle der Leistungsindikatoren durch den Betriebsleiter sind zum Teil erheblich, so dass in den meisten Fällen der Einsatz eines leistungsabhängigen Lohnsystems nicht wirtschaftlich sein wird. Der Einsatz des Precision Farmings macht die Anwendung der beschriebenen Leistungsindikatoren überhaupt erst möglich. Ohne moderne Datenerfassungen müsste der Betriebsleiter die für die Beurteilung der Arbeitsleistung notwendigen Parameter, wie z.B. die Fahrgeschwindigkeit, selbst erfassen. Aber auch mit dem Einsatz modernster Technik ist es nicht möglich, alle entscheidenden Arbeitsschritte zu überprüfen. Wenn es in der Zukunft denkbar ist, dass von technischer Seite alle für das Leistungslohnsystem notwendigen Kontrollen und Maschineneinstellungen im Ackerbau durchgeführt werden könnten, dann würden die Transaktionskosten der Betriebsleitung sinken. Hingegen wäre das Vergütungssystem dann durch hohe Investitionskosten belastet. Darüber hinaus wäre dann die Frage, wodurch sich Leistungsunterschiede bei den Mitarbeitern überhaupt noch ausdrücken könnten, da die Arbeitsleistung weitgehend durch die Maschine bestimmt wäre. Außerdem kann die Verwendung der beschriebenen Leistungsindikatoren $\mathrm{zu}$ zahlreichen Fehlanreizen führen, die sich in allen Produktionsschritten in einer schlechten Arbeitsqualität und einem hohen Maschinenverschleiß ausdrücken.

Die in Tabelle 32 dargestellten Parameter für Leistungslöhne, die sich an Kosten- und Leistungspositionen orientieren, sind zum Teil mit geringeren Transaktionskosten verbunden und implizieren weniger Fehlanreize.

Im Hinblick auf die theoretischen Vorüberlegungen lässt sich festhalten, dass die Verwendung besonders störanfälliger Leistungsindikatoren (z.B. die Entwicklung der Hektarerträge) dazu führen kann, dass die Bemessungsgrundlage des Leistungslohnsystems von den Mitarbeitern als ungerecht angesehen wird. Nach dem Motivationsmodell von PORTER UND LAWLER (vgl. Kapitel 3.2.4) würde dann die aus 
der individuellen Arbeitsleistung resultierende Belohnung aufgrund der wahrgenommenen Fairnessdefizite eine geringere Valenz haben, d.h., mittelfristig wäre das Leistungslohnsystem für die Mitarbeiter immer weniger attraktiv.

Aus den Ausführungen zu den möglichen Leistungsindikatoren im Ackerbau wird außerdem deutlich, dass diese zahlreiche Fehlanreize induzieren können. Es liegt daher nahe, eine Kombination verschiedener Indikatoren als Bemessungsgrundlage zu wählen und je nach ihrer ökonomischen Bedeutung zu gewichten. Diese Vorgehensweise führt aber unmittelbar zu komplexeren Abrechnungsmodalitäten, die für die Mitarbeiter weniger transparent als einfache Systeme sind. Die Erfolgsbedingung der zielorientierten Steuerung (vgl. Kapitel 3.4) würde dadurch leiden.

Betriebliches Wachstum im Ackerbau führt dazu, dass mehrere Mitarbeiter in den Produktionsprozess eingreifen. Es hängt von der Betriebsorganisation und der Anzahl der Mitarbeiter ab, welche Bedeutung leistungsorientierte Entlohnung im Ackerbau bei wachsenden Betriebsgrößen zukünftig haben wird. Die empirische Analyse zeigt, dass mit zunehmender Anzahl von Mitarbeitern die Betriebsleiter häufiger Erfahrung mit Leistungslöhnen gesammelt haben. In Betrieben mit einer sehr großen Anzahl von Beschäftigten im Ackerbau, die so organisiert sind, dass jedem Mitarbeiter eine eigene Maschine zugeordnet ist, ist die Anwendung einiger aufgeführter Leistungsindikatoren (z.B. die Unterschreitung der mehrjährigen Maschinenunterhaltungskosten) leichter als in Betrieben mit wenigen Mitarbeitern, die verschiedene Maschinen benutzen. In Lohnarbeitsbetrieben mit nur einem Mitarbeiter ist die Kontrolle der Bemessungsgrundlage hingegen mit geringeren Transaktionskosten verbunden als in größeren Betrieben, so dass hier keine allgemeingültige Aussage zur zukünftigen Bedeutung von Leistungslöhnen im Ackerbau getroffen werden kann. Es ist aber zu erwarten, dass selbst bei Einsatz des modernsten technischen Fortschritts die Störanfälligkeit der beschriebenen Leistungsindikatoren im Ackerbau nicht eliminiert werden kann, so dass der Zeitlohn in diesem Betriebszweig weiterhin die wichtigste Rolle spielen wird. 


\subsubsection{Leistungsindikatoren für die Milchproduktion}

Im Folgenden werden mögliche Leistungsindikatoren in der Milchproduktion anhand der verschiedenen Verfahrensschritte aufgezeigt. Die Leistungsindikatoren werden entlang des Lebens einer Milchkuh erläutert. Es sind nur solche Indikatoren aufgeführt, die kardinal messbar sind. Beispiele für subjektive Leistungskriterien in der Milchviehhaltung finden sich in Kapitel 5.4.2. Im Vergleich $\mathrm{zu}$ den Indikatoren im Ackerbau sind in der Tabelle 33 die notwendigen Kontrollmaßnahmen nicht aufgeführt, da die verwendeten Indikatoren mit Hilfe der üblichen Herdenmanagementprogramme, Aufzeichnungen und Auswertungen des Landeskontrollverbandes leicht berechnet werden können oder automatisch geliefert werden. Einzig die exakte Zuordnung des Grundfutters zu den Bereichen Kälber- und Jungviehaufzucht, Milchproduktion und Trockensteller und damit die Ermittlung der Grundfutterleistung ist in Betrieben schwieriger, die (noch) nicht mit dem Futtermischwagen füttern.

Vollkostenkalkulationen in der Milchproduktion zeigen, dass aus der Perspektive der Kostenrelevanz die Grund- und Kraftfutterkosten, die Personalkosten und die Remontierungskosten von besonderer Bedeutung sind (GOTTENSTRÄTER 2003: 14). Die Personalkosten kann der einzelne Mitarbeiter nur indirekt beeinflussen. Sie resultieren aus dem vereinbarten Lohn inklusive eventueller Leistungszuschläge und der dazugehörigen Sozialabgaben. Beeinflussbar sind nur die benötigten Arbeitskraftstunden pro Kuh, die aber vor allem durch die Betriebsorganisation und die Verfahrenstechnik bestimmt werden.

In der folgenden Tabelle 33 sind mögliche Leistungsindikatoren in der Milchproduktion dargestellt, die einen bedeutenden Einfluss auf die Wirtschaftlichkeit dieses Betriebszweigs haben und unter den Restriktionen und Besonderheiten des landwirtschaftlichen Produktionsprozesses als weitgehend objektiv messbar gelten können. 
TABELLE 33: MÖGLICHE LEISTUNGSINDIKATOREN IN DER MILCHPRODUKTION

\begin{tabular}{|c|c|c|c|c|c|c|}
\hline \multirow{2}{*}{$\begin{array}{l}\text { Verfahrens- } \\
\text { schritt }\end{array}$} & \multirow[t]{2}{*}{ Indikator } & \multirow{2}{*}{$\begin{array}{l}\text { Betriebliche Voraus- } \\
\text { setzungen }\end{array}$} & \multirow{2}{*}{$\begin{array}{c}\text { Mögliche } \\
\text { Fehlanreize }\end{array}$} & \multicolumn{2}{|c|}{ Bewertung von } & \multirow{2}{*}{$\begin{array}{l}\text { Vorzüglich- } \\
\text { keit }\end{array}$} \\
\hline & & & & $\begin{array}{c}\text { Höhe der } \\
\text { Transaktionskosten }\end{array}$ & $\begin{array}{c}\text { Stör- } \\
\text { anfälligkeit des } \\
\text { Leistungsindikators }\end{array}$ & \\
\hline Kalbung & Kälberverluste in \% & $\begin{array}{l}\text { Kein unverschuldeter } \\
\text { Krankheitseinbruch im Bestand }\end{array}$ & $\begin{array}{l}\text { Unnötige prophylaktische } \\
\text { tierärztliche } \\
\text { Behandlungen }\end{array}$ & $\downarrow$ & $\uparrow$ & 3 \\
\hline Aufzucht & Erstkalbealter in Monaten & $\begin{array}{l}\text { Kontrolle des notwendigen } \\
\text { Lebendgewichts bei } \\
\text { Erstbesamung }\end{array}$ & $\mathrm{Zu}$ frühe Besamung & $\downarrow$ & $\uparrow$ & 6 \\
\hline Fütterung & $\begin{array}{l}\text { Kraftfuttereinsatz in } \mathrm{kg} / \mathrm{kg} \\
\text { Milch, } \\
\text { Grundfutterleistung in } \mathrm{kg}\end{array}$ & $\begin{array}{l}\text { Zuordnung der verbrauchten } \\
\text { Futtermengen zu den } \\
\text { Tiergruppen, } \\
\text { Mitarbeiter in der Milch- } \\
\text { produktion müssen auch für den } \\
\text { Futterbau zuständig sein }\end{array}$ & $\begin{array}{l}\text { Der Milchleistung nicht } \\
\text { angepasster } \\
\text { Kraftfuttereinsatz }\end{array}$ & $\begin{array}{l}\rightarrow \\
\downarrow\end{array}$ & $\begin{array}{l}\rightarrow \\
\uparrow\end{array}$ & 2 \\
\hline Milchproduktion & $\begin{array}{l}\text { Milchleistung in kg ECM, } \\
\text { Zellzahlen, } \\
\text { Milchgüteklasse }\end{array}$ & $\begin{array}{l}\text { Erfassung der Indikatoren durch } \\
\text { Milchkontrolle, } \\
\text { Herdenmanagementprogramme, } \\
\text { Buchführung }\end{array}$ & $\begin{array}{l}\text { Zu hoher } \\
\text { Kraftfuttereinsatz, } \\
\text { kürzere Nutzungsdauer }\end{array}$ & $\downarrow$ & $\uparrow$ & 1 \\
\hline $\begin{array}{l}\text { Abstand zwischen } \\
\text { Kalbungen }\end{array}$ & $\begin{array}{l}\text { Zwischenkalbezeit in } \\
\text { Tagen, } \\
\text { Besamungsindex }\end{array}$ & $\begin{array}{l}\text { Mitarbeiter hat Verantwortung } \\
\text { für Herdenmanagement } \\
\text { Eigenbestandsbesamung }\end{array}$ & Verkürzung der Laktation & $\downarrow$ & $\rightarrow$ & 4 \\
\hline Remontierung & Remontierungsrate in \% & $\begin{array}{l}\text { Mitarbeiter hat Verantwortung } \\
\text { für Herdenmanagement }\end{array}$ & $\begin{array}{l}\text { Remontierungsrate wird } \\
\text { künstlich zu niedrig } \\
\text { gehalten, optimale } \\
\text { Nutzungsdauer der Kuh } \\
\text { wird überschritten }\end{array}$ & $\downarrow$ & $\uparrow$ & 5 \\
\hline
\end{tabular}

Quelle: Eigene Darstellung 
Die „Kälberverluste in Prozent““ können als ein Parameter zur Bemessung von Leistungslöhnen dienen. Mitarbeiter, die eine Prämie für geringe Kälberverluste im Vergleich zu einem Normwert, der auf betrieblichen Erfahrungen oder Betriebszweigabrechnungen basiert, erhalten, sollen dadurch motiviert werden, die Betreuung der Kälber zu verbessern. Der Parameter ist störanfällig gegenüber Krankheitseinbrüchen im Bestand. Im Hinblick auf das Erfolgskriterium Zielkompatibilität können unter Umständen Zielkonflikte mit dem Oberziel Gewinnmaximierung auftreten, wenn die Tierarzt- bzw. Medikamentenkosten durch die Anwendung des Parameters steigen. Dieses Problem kann dann auftreten, wenn die Mitarbeiter die Verantwortung für den Betriebszweig Milchproduktion haben und damit auch die Befugnis haben, den Tierarzt zu bestellen.

Als Erfolgskennziffer für den Bereich Aufzucht ist das „Erstkalbealter in Monaten“ von Bedeutung. Betriebswirtschaftliche Auswertungen zeigen, dass pro Monat spätere Kalbung durchschnittlich zwischen 30 und $50 €$ Aufzuchtkosten einzukalkulieren sind (GOTTENSTRÄTER 2003: 14, RINDERREPORT RHEINLAND-PFAlZ 2007, LANDWIRTSCHAFTSKAMMER SCHLESWIG-HOLSTEIN 2005a: 18). Wird aus dem eigenen Bestand remontiert, so könnte das Erstkalbealter in Monaten als Leistungskriterium herangezogen werden. Es eignet sich jedoch nur für verantwortliche Leitungskräfte und nicht für Arbeiter in der Produktion ohne Verantwortungsbereich, da es unter anderem von der Intensität der Kälberaufzucht bestimmt wird und deshalb nur von Mitarbeitern maßgeblich beeinflusst werden kann, die Verantwortung für das Herdenmanagement tragen. Außerdem ist darauf $\mathrm{zu}$ achten, dass das frühere Erstkalbealter mit einer Intensivierung der Aufzucht einhergeht. Erreichen die Färsen zum Zeitpunkt der Erstbesamung nicht das notwendige Lebendgewicht, dann wäre eine Reduzierung des Erstkalbealters kontraproduktiv für die Wirtschaftlichkeit des Betriebszweigs. Die Erfolgsbedingung der Zielkompatibilität (vgl. Kapitel 3.4) wäre nicht mehr gegeben. Die befragten Betriebsleiter setzen das Erstkalbealter nicht als Leistungsindikator ein (vgl. Kapitel 5.4.2). Dies könnte ein Hinweis darauf sein, dass entweder die betrieblichen Voraussetzungen für die Verwendung dieses Parameters nicht gegeben sind oder dass er als zu störanfällig angesehen wird.

Für die Wirtschaftlichkeit der Milchviehhaltung sind aber auch die Effizienz des Kraftfuttereinsatzes und die Grundfutterleistung von entscheidender Bedeutung. Die „Kraftfuttereffizienz“ kann in der Menge Kraftfutter (in g oder kg) gemessen werden, 
die notwendig ist, $\mathrm{um}$ ein $\mathrm{kg}$ Milch $\mathrm{zu}$ erzeugen. Auswertungen der Milchviehspezialberatung Rheinland-Pfalz zeigen, dass die $25 \%$ besseren Betriebe weniger Kraftfutter pro Kuh, dafür aber etwas teureres Kraftfutter einsetzen (RINDERREPORT RHEINLAND-PFAlz 2007). Der Kontrollaufwand und die Störanfälligkeit dieses Parameters sind als mittelhoch einzuschätzen. Eine mögliche Schwachstelle dieses Indikators ist der Fehlanreiz Kraftfutter zu sparen und gleichzeitig weniger Milch zu produzieren. Es muss folglich sichergestellt werden, dass von den Mitarbeitern auch eine Steigerung der Milchleistung angestrebt wird. Horizontale und vertikale Betriebsvergleiche können zur sachgerechten Interpretation der erreichten Kennzahl beitragen. Ein effizienter Kraftfuttereinsatz in der Milchviehhaltung steht in enger Verbindung zur Grundfutterleistung.

Die „Grundfutterleistung“ ist die Menge Milch, die aus dem Grundfutter ermolken wird. Sie wird durch das genetische Potenzial der Kuh (Großrahmigkeit, Grundfutteraufnahmevermögen), das Fütterungsmanagement und die Grundfutterqualität (Energiedichte, Trockenmassegehalt, Schmackhaftigkeit) wesentlich beeinflusst. Die Grundfutterleistung kann leicht gemessen werden. Sie ist jedoch aufgrund der Vielzahl der sie beeinflussenden Faktoren störanfällig. Dieser Parameter kann nicht eingesetzt werden, wenn die für die Milchproduktion verantwortlichen Mitarbeiter nicht für die Futterwerbung zuständig sind. In landwirtschaftlichen Betrieben, in denen die für den Ackerbau verantwortlichen Mitarbeiter ebenfalls für den Futterbau zuständig sind, ist der Einsatz dieses Leistungskriteriums deshalb nicht sinnvoll. Diese Einschränkung verdeutlicht, warum die Grundfutterleistung als Leistungskriterium in den befragten Betrieben nur eine geringe Rolle spielt.

Das genetische Potenzial zur Milchleistungssteigerung liegt nach GOTTENSTRÄTER (2003: 13) bei circa $1.000 \mathrm{~kg}$ pro Kuh. Um einen Anreiz zum Ausschöpfen dieser Reserven zu geben, kann die „Milchleistung“ als Entlohnungsparameter herangezogen werden. Die empirische Analyse zeigt, dass die Milchleistung in den analysierten Betrieben häufig als Bemessungsgrundlage für Leistungslöhne herangezogen wird. Qualitätsindikatoren wie die „,erreichte Milchgüteklasse“ oder die „Zellzahlen“ sollten mit berücksichtigt werden, denn sie haben einen hohen Informationsgehalt über die Arbeitsqualität der Mitarbeiter in Bezug auf die Eutersauberkeit. Zwar sind Qualitätsparameter nicht unbeeinflusst von Krankheitseinbrüchen in der Herde; sie 
können aber dazu beitragen, dass das Bewusstsein der Mitarbeiter für die Bedeutung der Milchqualität geschärft wird und Eutererkrankungen frühzeitig erkannt werden. Der Kontrollaufwand für diese Parameter ist gering, da die notwendigen Daten durch die Milchkontrolle und Milchgeldabrechnung automatisch zur Verfügung stehen. Die Zuordnung der Leistung zu den einzelnen Mitarbeitern ist dann schwierig, wenn verschiedene Mitarbeiter für die gleichen Aufgaben zuständig sind, zum Beispiel wenn im Schichtsystem gearbeitet wird. Aus diesem Grund wenden Betriebe, die ihre Mitarbeiter in der Milchproduktion leistungsorientiert entlohnen, häufig eine Gruppenvergütung an, wie die empirischen Ergebnisse zeigen (vgl. Kapitel 5.4.1).

Eine weitere wichtige Determinante für die Wirtschaftlichkeit der Milchproduktion ist die „Zwischenkalbezeit“. Sie gibt Hinweise auf die Qualität der Brunstbeobachtung. Zwar sinken die Fruchtbarkeitsleistungen bei Hochleistungsherden insgesamt ab, trotzdem zeigen Betriebsvergleiche auch bei ähnlichen Milchleistungen deutliche Unterschiede zwischen den Betrieben. Nach Ergebnissen der Milchviehspezialberatung Rheinland-Pfalz erzielten die $25 \%$ besseren Betriebe im Wirtschaftsjahr 2004/2005 eine Zwischenkalbezeit von 402 Tagen während die $25 \%$ schlechteren Betriebe im gleichen Zeitraum eine Zwischenkalbezeit von 414 Tagen erreichten (RINDERREPORT RHEINLAND-PFALZ 2007: 41). Als weiteres Kriterium kann der „Besamungsindex“ dienen. Er bietet sich vor allem für Besamungstechniker und Eigenbestandsbesamer an. Der Kontrollaufwand beider Parameter ist gering und die Störanfälligkeit als durchschnittlich einzuschätzen. Die Störanfälligkeit wird nicht als niedrig eingeschätzt, da die Fruchtbarkeitsleistung von verschiedenen Faktoren beeinflusst wird.

Die „Remontierungsrate in \%“ gibt Auskunft über die Nutzungsdauer der Milchkuh. Betriebsvergleiche zeigen, dass viele Milchkühe eine zu kurze Lebensleistung erreichen und kaum die Kosten für die Ersatzfärse erwirtschaften (LANDWIRTSCHAFTSKAMMER Schleswig-Holstein 2005a: 25). Die Nutzungsdauer der Milchkuh wird durch die Faktoren Fütterung, Haltung (Kuhkomfort) und Herdenmanagement bestimmt (ebenda: 25). Wird die Remontierungsrate als Leistungsindikator herangezogen, so ist darauf zu achten, dass der betreffende Mitarbeiter diese Faktoren auch beeinflussen kann. Dies ist vorrangig bei Herdenmanagern der Fall.

Die Erörterung der Vor- und Nachteile der Leistungsindikatoren in der Milchproduktion verdeutlicht, dass die einzelnen Verfahrensschritte und ihre produktionstechnischen und 
wirtschaftlichen Kennzahlen starken Wechselwirkungen unterliegen. Im Ackerbau konnte jeder Arbeitschritt als eigenständiger Arbeitsgang angesehen werden, obwohl auch hier Interdependenzen bestehen. Für die leistungsorientierte Entlohnung in der Milchproduktion lässt sich daraus schlussfolgern, dass diese als eine Einheit gesehen werden muss und sich folglich die Vergütung nach Leistung entweder an einem aggregierten Parameter wie der Milchleistung und -qualität orientieren, oder mehrere Indikatoren als Bemessungsgrundlage zusammengefasst werden sollten. Die Verwendung mehrerer Indikatoren führt jedoch zu höheren Transaktionskosten und negativen Wirkungen auf die Erfolgsbedingung der zielorientierten Steuerung, wie bereits für den Ackerbau erläutert wurde.

Betriebliches Wachstum und Precision Agriculture werden auch in der Milchproduktion die Rahmenbedingungen für leistungsabhängige Vergütungsformen verändern. Mit zunehmender Anzahl von Mitarbeitern wird die Messung der individuellen Arbeitsleistung komplexer. Die Ausführungen zum Prinzipal-Agenten-Problem (Kapitel 3.1.1) zeigten, dass dadurch der Anreiz zum „,bummeln“ bei den einzelnen Mitarbeitern erhöht wird. Die Wahrscheinlichkeit, dass ein Betriebsleiter leistungsabhängig entlohnt, um dieses zu verhindern, steigt folglich. Da die Bemühung des Einzelnen in großen Milchviehanlagen schwer messbar ist oder durch den Einsatz der Kollegen überlagert werden kann, ist es wahrscheinlich, dass bei steigender Betriebsgröße die Gruppenvergütung eine größere Bedeutung erlangt. Die Vor- und Nachteile von Teamvergütungen wurden in Kapitel 4.2.4 dargestellt, praktisch hat diese Vergütungsform bereits Relevanz, wie die Ergebnisse der Betriebsleiterbefragung zeigen.

Der Einsatz moderner Melk- und Fütterungstechnik trägt schon heute zur automatischen Erfassung wichtiger produktionstechnischer Kennzahlen bei. Technische Unterstützung in den Melkständen, hier ist z.B. die automatische Abnahme der Melkzeuge zum Ende des Melkvorgangs zu nennen, entlastet die melkende Person und erleichtert den Arbeitsprozess. Trotz des technischen Fortschritts während des Melkens verbleiben wichtige Vorbereitungs- (z.B. Euterreinigung) und Kontrollarbeiten (Eutergesundheit) in der Hand des Personals, so dass die Leistung der Beschäftigten nach wie vor einen großen Einfluss auf die Milchleistung und -qualität hat. Melkroboter entbinden Betriebsleiter und Lohnarbeitskräfte von zeitlich festgelegten Melkzeiten und schaffen damit mehr Flexibilität im Arbeitsprozess. Mit dem Einsatz automatischer Melksysteme 
ändert sich das Aufgaben- und Anforderungsprofil für die Beschäftigten in der Milchproduktion erheblich. Für die Überwachung des erfolgreichen Melkvorgangs sind mehr technische Fähigkeiten und kontrollierende Fähigkeiten notwendig. Die bisherige Verbreitung von Melkrobotern zeigt, dass diese vorrangig in Ländern mit Familienarbeitsverfassung eingesetzt werden (HARMS 2004: 17). Mit zunehmender Betriebsgröße ist folglich nicht zu erwarten, dass automatische Melksysteme eine große Bedeutung erlangen. Dies zeigt auch eine Untersuchung zu den Wachstumsoptionen landwirtschaftlicher Familienbetriebe im Kontext technologischen Fortschritts. Der überwiegende Teil der in dieser Untersuchung befragten wachstumswilligen Landwirte lehnte den Einsatz von Melkrobotern ab (SCHAPER 2006: 121, DAVIER ET AL. 2006: 34).

\subsubsection{Leistungsindikatoren für die Sauenhaltung und Schweinemast}

In diesem Abschnitt sollen Leistungsindikatoren für die Schweineproduktion ausgewiesen werden, die als Bemessungsgrundlage für eine leistungsorientierte Entlohnung herangezogen werden können.

\subsubsection{Leistungsindikatoren für die Sauenhaltung}

Zunächst werden verschiedene Parameter für die Sauenhaltung aufgezeigt. Im Anschluss daran wird auf mögliche Parameter für die Schweinemast eingegangen. 
TABELLE 34: LEISTUNGSINDIKATOREN FÜR DIE SAUENHALTUNG

\begin{tabular}{|c|c|c|c|c|c|c|}
\hline \multirow{2}{*}{$\begin{array}{l}\text { Verfahrens- } \\
\text { schritt }\end{array}$} & \multirow[t]{2}{*}{ Indikator } & \multirow{2}{*}{$\begin{array}{l}\text { Betriebliche Voraus- } \\
\text { setzungen }\end{array}$} & \multirow[t]{2}{*}{ Mögliche Fehlanreize } & \multicolumn{2}{|c|}{ Bewertung von } & \multirow{2}{*}{$\begin{array}{l}\text { Vorzüglich- } \\
\quad \text { keit. }\end{array}$} \\
\hline & & & & $\begin{array}{l}\text { Höhe der } \\
\text { Transaktions- } \\
\text { kosten } \\
\end{array}$ & $\begin{array}{c}\text { Stör- } \\
\text { anfälligkeit des } \\
\text { Leistungsindikators } \\
\end{array}$ & \\
\hline Abferkeln & $\begin{array}{l}\text { lebendig geborene } \\
\text { Ferkel pro Sau }\end{array}$ & keine & $\begin{array}{l}\text { Zu hohe Remontierung, da die } \\
\text { Wurfgrößen von Jungsauen größer } \\
\text { sind als bei Altsauen }\end{array}$ & $\downarrow$ & $\uparrow$ & 2 \\
\hline Aufzucht & $\begin{array}{l}\text { Ferkelverluste in } \% \\
\text { aufgezogene Ferkel pro } \\
\text { Sau und Jahr }\end{array}$ & $\begin{array}{l}\text { Kein unverschuldeter } \\
\text { Krankheitseinbruch im Bestand }\end{array}$ & $\begin{array}{l}\text { Unnötige prophylaktische } \\
\text { tierärztliche Behandlungen } \\
\mathrm{Zu} \text { hohe Remontierungsrate }\end{array}$ & $\begin{array}{l}\downarrow \\
\downarrow\end{array}$ & $\begin{array}{l}\uparrow \\
\uparrow\end{array}$ & 1 \\
\hline Fütterung & $\begin{array}{l}\text { Ferkelfutter in } \mathrm{kg} / \text { Ferkel } \\
\text { Sauenfutter in } \mathrm{kg} / \mathrm{Sau}\end{array}$ & $\begin{array}{l}\text { Betriebliche Aufzeichnungen } \\
\text { der verbrauchten Mengen }\end{array}$ & $\begin{array}{l}\text { Futtermenge an den Bedarf von } \\
\text { Ferkeln und Sauen nicht angepasst }\end{array}$ & $\vec{\downarrow}$ & $\vec{\uparrow}$ & 5 \\
\hline Wurffrequenz & $\begin{array}{l}\text { Zwischenwurfzeit in } \\
\text { Tagen }\end{array}$ & $\begin{array}{l}\text { Betriebliche Auswertung der } \\
\text { Zwischenwurfzeit durch } \\
\text { Herdenmanagementprogramme } \\
\text { (Sauenplaner) }\end{array}$ & $\begin{array}{l}\text { Zu kurze Zwischenwurfzeit, } \\
\text { Verkürzung der Laktation }\end{array}$ & $\downarrow$ & $\rightarrow$ & 3 \\
\hline Remontierung & Remontierungsrate in $\%$ & $\begin{array}{l}\text { Mitarbeiter hat Verantwortung } \\
\text { für Herdenmanagement } \\
\text { Eigenbestandsbesamung }\end{array}$ & $\begin{array}{l}\text { Remontierungsrate wird künstlich } \\
\text { zu niedrig gehalten, optimale } \\
\text { Nutzungsdauer der Sau wird } \\
\text { überschritten }\end{array}$ & $\downarrow$ & $\uparrow$ & 4 \\
\hline
\end{tabular}

Quelle: Eigene Darstellung 
Entscheidend für den Erfolg des Betriebszweigs Sauenhaltung ist die Kennzahl „aufgezogene Ferkel pro Sau und Jahr“". Sie ist Ausdruck für die Qualität der Produktionstechnik und die Leistungsfähigkeit des Tierbestandes (LANDWIRTSCHAFTSKAMMER SCHLESWIG-HOLSTEIN 2005a: 62) und kann als aggregierter Parameter für eine leistungsorientierte Entlohnung dienen. Die große Bedeutung dieser Schlüsselkennzahl belegt auch die durchgeführte empirische Analyse (vgl. Kapitel 5.4.2). Sie wird von verschiedenen Faktoren beeinflusst, die sich zum Teil auch als Kennzahlen für eine variable Entlohnung eignen und in Abhängigkeit von den betrieblichen Schwachstellen als Indikator ausgewählt werden können (vgl. Tabelle 34).

Zunächst einmal ist die Kennzahl „Anzahl der lebendig geborenen Ferkel je Wurf“ entscheidend. Dieser Parameter ist leicht kontrollierbar, jedoch relativ störanfällig, da er bestimmt wird von der Anzahl der Ferkel, die geboren werden und von der Anzahl der Ferkel, die bei der Geburt sterben. Das Alter der Sau, das genetische Potenzial des Tierbestandes und die Fütterung beeinflussen die Anzahl der Ferkel pro Trächtigkeit. Die Anzahl der Ferkel, die perinatal sterben, wird von der Betreuungsintensität während des Ferkelns beeinflusst (LANDWIRTSCHAFTSKAMMER SCHLESWIG-HOLSTEIN 2005a: 62). Nach Ergebnissen der LANDWIRTSCHAFTSKAMMER SCHLESWIG-HOLSTEIN wird die Anzahl aufgezogener Ferkel pro Sau und Jahr von der Wurfgröße weniger stark als von den Ferkelverlusten in \% bis zum Absetzen beeinflusst (ebenda: 62).

Den größten Einfluss auf die oben genannte Kennziffer hat jedoch die Wurffrequenz. Hier zeigen sich noch deutliche Unterschiede zwischen den Sauenbetrieben (ebenda: 62). Durch eine Verkürzung der Zwischenwurfzeit kann in den weniger erfolgreichen Betrieben die Anzahl der abgesetzten Ferkel pro Sau entscheidend erhöht werden, so dass dieser Parameter ebenfalls ein geeigneter Parameter für eine leistungsorientierte Entlohnung sein kann. Die Vielzahl der Faktoren, die die Erfolgskennzahl der aufgezogenen Ferkel pro Jahr beeinflusst, verdeutlicht die Störanfälligkeit dieses Leistungsindikators. Eine Praxisempfehlung lautet daher für den Einsatz von Leistungslöhnen in der Schweineproduktion, Leistungszulagen und Prämien bei Krankheitseinbrüchen auf einen Zeitraum von drei bis vier Monaten vor dem Krankheitseinbruch zu beziehen (GRIESEL 2004: 6).

Auf der Kostenseite beeinflussen die Futterkosten die Produktionskosten stark. Mit dem Produktivitätsanstieg in den Betrieben nimmt auch der Futterverbrauch in kg pro Ferkel 
und Sau tendenziell zu. Jedoch verbrauchen die erfolgreicheren Betriebe nicht unbedingt mehr Futter für Ferkel und Sauen, so dass die Futtereffizienz ebenfalls ein Kriterium für die Qualität des Managements und der Produktionstechnik ist (LANDWIRTSCHAFTSKAMMER SCHLESWIG-HOLSTEIN 2005a: 64).

Die Bestandsergänzung ist, wie auch in der Milchproduktion, eine weitere wichtige Kennzahl für die Leistung des Betriebszweigs Sauenhaltung. Die erfolgreichen Betriebe der Betriebszweigauswertung der Landwirtschaftskammer Schleswig-Holstein wiesen im WJ 2003/2004 eine um durchschnittlich drei Prozentpunkte geringere Remontierungsrate als die weniger erfolgreichen Betriebe auf (ebenda: 67). Eine Erhöhung der aufgezogenen Ferkel pro Sau und Jahr führt folglich nicht zwangsläufig zu einem Anstieg der Remontierungsrate. GRIESEL (2004: 7) empfiehlt, die Remotierungsrate in ein leistungsabhängiges Vergütungsschema einzubeziehen, wenn deutlich wird, dass ein Mitarbeiter versucht, die Anzahl der aufgezogenen Ferkel pro Sau und Jahr mit Hilfe einer überhöhten Remontierung zu erreichen.

\subsubsection{Leistungsindikatoren für die Schweinemast}

Die direktkostenfreie Leistung in der Schweinemast als Kennziffer für die Wirtschaftlichkeit des Betriebszweigs Schweinemast unterliegt zwischen den Jahren starken Schwankungen. Sie wird besonders stark von der Höhe der Mastschweine- und Ferkelpreise beeinflusst. Eine Beteiligung der Mitarbeiter an der direktkostenfreien Leistung unterwirft auch die Einkommen der Mitarbeiter starken, zum großen Teil marktgegebenen Einflüssen. Zwischen den Betrieben zeigen sich deutliche Unterschiede in den biologischen und ökonomischen Leistungen, die auf produktionstechnische und unternehmerische Einflüsse zurückzuführen sind (LANDWIRTSCHAFTSKAMMER SCHLESWIG-HOLSTEIN 2005a: 72-73).

In der folgenden Tabelle 35 sind die Parameter aufgeführt, die im Laufe des Produktionsprozesses die direktkostenfreie Leistung stark beeinflussen und die als Indikator für eine leistungsorientierte Entlohnung herangezogen werden können. 
TABELLE 35: LEISTUNGSINDIKATOREN FÜR DIE SCHWEINEMAST

\begin{tabular}{|l|l|c|c|}
\hline \multirow{2}{*}{ Verfahrensschritt } & \multicolumn{1}{|c|}{ Indikator } & \multicolumn{2}{|c|}{ Bewertung von } \\
\cline { 3 - 4 } & & $\begin{array}{c}\text { Höhe der } \\
\text { Transaktionskosten }\end{array}$ & $\begin{array}{c}\text { Stör- } \\
\text { anfälligkeit des } \\
\text { Leistungsindikators }\end{array}$ \\
\hline Fütterung & Tägliche Zunahmen in g & $\downarrow$ & $\rightarrow$ \\
\hline $\begin{array}{l}\text { Verkaufte } \\
\text { Schweine/Betrieb }\end{array}$ & Mastschweineverluste in \% & $\downarrow$ & $\uparrow$ \\
\hline $\begin{array}{l}\text { Schlachtkörper- } \\
\text { qualität }\end{array}$ & Magerfleischanteil in \% & $\downarrow$ & $\downarrow$ \\
\hline
\end{tabular}

Quelle: Eigene Darstellung

Als Indikator für die Qualität der Produktionstechnik und des Herdenmanagements können die täglichen Zunahmen dienen. Nach Berechnungen der LANDWIRTSCHAFTSKAMMER SCHLESWIG-HOLSTEIN drücken sich hohe tägliche Zunahmen in einer guten Futterverwertung und einem geringeren Futterverbrauch je kg Zuwachs aus. Die erfolgreichen Betriebe unterscheiden sich von den weniger erfolgreichen Betrieben durch bis zu 5 Cent pro $\mathrm{kg}$ Zuwachs niedrigere Futterkosten (ebenda: 73). Der Kontrollaufwand zur Bemessung des Parameters ist gering. Die Störanfälligkeit ist mittelhoch, da die täglichen Zunahmen von mehreren Faktoren abhängen und beispielsweise technische Probleme mit der Temperatur und Lüftung im Stall, die nicht durch den Mitarbeiter verursacht sein müssen, sich direkt in der Leistung widerspiegeln. Für die Anzahl der verkauften Schweine pro Betrieb sind bei einer gegebenen Ausstattung an Mastschweineplätzen neben den täglichen Zunahmen, die die Mastdauer beeinflussen, die Mastschweineverluste von Bedeutung. Die Mastschweineverluste sind ein einfach zu messender Leistungsindikator, der jedoch störanfällig gegenüber Krankheitseinbrüchen und der Ferkelherkunft ist. Im Sinne der Zielkompatibilität können bei alleiniger Verwendung dieses Parameters Zielkonflikte mit der Gewinnmaximierung eintreten, wenn hierdurch ein Fehlanreiz zu überhöhten Tierarzt- und Medikamentenkosten gegeben wird.

Die erfolgreichen und weniger erfolgreichen Betriebe unterscheiden sich im erzielten Verkaufspreis. Die Preisdifferenz wird hier vor allem durch die Absatzmenge und einen unterschiedlich hohen Magerfleischanteil begründet (LANDWIRTSCHAFTSKAMMER SCHLESWiG-Holstein 2005a: 72). Auf die Absatzmenge hat der Mitarbeiter nur einen geringen Einfluss, da sie im Betrieb vorgegeben ist. Den Magerfleischanteil als 
Leistungsindikator für die Vergütung zugrunde zu legen, ist problematisch, da die genetische Herkunft der Tiere einen großen Einfluss auf den Magerfleischanteil hat.

\subsubsection{Zusammenfassende Beurteilung der Leistungsindikatoren für die Schweinehaltung}

Aus den Ausführungen zu den möglichen Leistungsindikatoren in der Sauenhaltung und in der Schweinemast wird deutlich, dass ein Leistungslohnsystem nur für Mitarbeiter mit Verantwortung für das Herdenmanagement und die Produktionstechnik geeignet ist.

Steigende Tierbestände in der Sauenhaltung und Schweinemast werden dazu führen, dass in den Großbetrieben mehrere Mitarbeiter für die Betreuung der Tiere zuständig sein müssen, um den Arbeitsbedarf zu bewältigen. Die Auswertungen der SÄCHSISCHEN LANDESANSTALT FÜR LANDWIRTSCHAFT (2005: 21) zeigen, dass in sächsischen Großbetrieben durchschnittlich 0,62 AKh pro Mastschwein und 18,3 AKh pro Sau ab der Erstbelegung im Jahr benötigt werden. Eine Arbeitskraft kann - unter Berücksichtung einer jährlich verfügbaren Arbeitszeit von $1.900 \mathrm{AKh}-$ folglich ungefähr 1.261 Mastschweine und 111 Sauen betreuen. Daraus lässt sich für die analysierten Veredlungsbetriebe der Arbeitgeberbefragung (vgl. Kapitel 5.2.1) schlussfolgern, dass durchschnittlich 5,5 Arbeitskräfte in der Mastschweinehaltung und knapp fünf Arbeitskräfte in der Sauenhaltung beschäftigt sind. Dies führt zu ähnlichen Herausforderungen an die Lohngestaltung wie in der Milchproduktion, d.h. Gruppenvergütungen werden eine zunehmende Bedeutung erlangen. Größere betriebliche Einheiten können aber auch eine stärkere Arbeitsteilung nach sich ziehen. Wenn mehr Leitungskräfte in der Tierproduktion eingesetzt werden, steigen die Chancen für leistungs- und erfolgsorientierte Entlohnungssysteme, da Mitarbeiter mit Verantwortung für das Herdenmanagement die oben beschriebenen Kennziffern stärker beeinflussen können als Mitarbeiter mit rein ausführenden Aufgabenfeldern.

Die aktuelle Auswertung der DLG-Spitzenbetriebe der Sauenhaltung zeigt einen deutlichen Trend zur Wachstumsbereitschaft, vor allem in den alten Bundesländern. In den nächsten fünf Jahren möchten die Betriebsleiter aus den südwestdeutschen Bundesländern eine Stallkapazität von durchschnittlich 312 Sauenplätzen erreicht haben. Die nordwestdeutschen Landwirte wollen zum gleichen Zeitpunkt im Mittel über 404 Sauenplätze verfügen und die ostdeutschen Mitglieder des DLG-Forums 
Spitzenbetriebe streben eine Konsolidierung ihres Sauenbestandes bei Verbesserung der biologischen Leistungen an (ZIRON 2007).

In Bezug auf den Einsatz technischen Fortschritt kann festgehalten werden, dass die Automatisierung der Fütterung in der Schweinehaltung mit wachsenden Tierbeständen einhergeht. Der züchterische Fortschritt gemeinsam mit automatisierter Fütterung senkt den Einflussbereich der Mitarbeiter in der Schweinehaltung und hier insbesondere in der Schweinemast. Betreuungsaufgaben, wie z.B. die Geburtenüberwachung, der Wurfausgleich in der Ferkelproduktion und Maßnahmen zur Sicherung der Tiergesundheit werden aber auch in Zukunft nicht durch Maschinen ersetzt werden können. Sie bieten auch zukünftig den Ansatzpunkt für Leistungslohnsysteme.

\subsubsection{Leistungsindikatoren für die Biogas- und Stromproduktion}

Die Bereitstellung regenerativer Energie entwickelt sich für viele landwirtschaftliche Betriebe $\mathrm{zu}$ einem wichtigen Betriebszweig, dessen Betrieb mit neuen Herausforderungen verbunden ist. Aus diesem Grund soll exemplarisch für die Biogaserzeugung die Möglichkeit einer leistungsorientierten Entlohnung der Mitarbeiter diskutiert werden. Dabei werden auch Indikatoren zur Bemessung eines leistungsabhängigen Arbeitsentgelts aufgezeigt.

Biogasanlagen werden in Deutschland häufig als landwirtschaftlicher Nebenbetrieb oder als eigenständige Gesellschaft betrieben (DLG 2006: 21). Zur Verrichtung der täglichen Arbeit werden entweder Lohnarbeits- oder Familienarbeitskräfte eingesetzt. Betreiben mehrere Landwirte gemeinsam eine Biogasanlage, so erfüllen die Landwirte, die für die tägliche Arbeit an der Biogasanlage verantwortlich sind, die Funktion von Lohnarbeitskräften und werden von der Betreibergesellschaft auch entsprechend entlohnt. Neben einer festen Vergütung kommt folglich auch bei dieser Personengruppe eine leistungsorientierte Vergütung in Frage. Vorab ist $\mathrm{zu}$ sagen, dass ein Leistungslohnsystem erst eingeführt werden kann, wenn die Biogasanlage hochgefahren ist, ein kontinuierlicher Volllastbetrieb erreicht worden ist und technische Anlaufschwierigkeiten überwunden sind.

Aus der Neuheit dieses Betriebszweiges resultiert die Schwierigkeit, dass es bisher kaum Daten über die in der Praxis erreichten Kennzahlen und ökonomischen Ergebnisse 
gibt (ebenda: 8). Belastbare Betriebsvergleiche müssen erst mit der Zeit gewonnen werden.

Die Perspektive einer leistungsorientierten Entlohnung in Biogasanlagen ist insofern günstig, als dass der Produktpreis in Cent/kWh nicht vom Markt bestimmt wird, sondern durch das Erneuerbare-Energien-Gesetz vom 1. August 2004 gesetzlich festgeschrieben ist. Die Mitarbeiter sind dadurch nicht von Preisschwankungen betroffen, so dass auch Erfolgsbeteiligungen in Frage kommen können. Außerdem ist es dadurch für die Betriebsleiter leichter, eine Wirtschaftlichkeitsberechnung des Leistungslohnsystems durchzuführen, da der Produktpreis ex ante feststeht.

In Analogie zu den Empfehlungen der DLG-Arbeitsgruppe Biogas soll der Betriebszweig Biogas die Verfahrensschritte Biogaserzeugung, -verwertung und Gärrestverwertung umfassen. Da es beim Betrieb einer Biogasanlage unterschiedliche Schnittstellen zwischen der Substraterzeugung und -verwertung gibt, soll als Annahme gelten, dass die Substrate dem Mitarbeiter, der die Biogasanlage betreut, geliefert werden.

In Tabelle 36 sind wichtige Erfolgskennziffern der Biogaserzeugung aufgeführt, die im Fermenter und/oder im Blockheizkraftwerk gemessen werden können und als Indikator für ein Leistungslohnsystem dienen können.

TABELLE 36: MÖGLICHE LEISTUNGSINDIKATOREN IN BIOGASANLAGEN

\begin{tabular}{|l|l|c|c|}
\hline Verfahrensschritt & \multicolumn{1}{|c|}{ Indikator } & \multicolumn{2}{|c|}{ Bewertung von } \\
\cline { 3 - 4 } & & \multicolumn{1}{|c|}{$\begin{array}{c}\text { Höhe der } \\
\text { Transaktions- } \\
\text { kosten }\end{array}$} & $\begin{array}{c}\text { Stör- } \\
\text { anfälligkeit des } \\
\text { Leistungsindikators }\end{array}$ \\
\hline \multirow{2}{*}{ Fermentierung } & $\begin{array}{l}\text { Soll-Ist Biogas Nm }{ }^{3} \text { Vergleich } \\
\text { geplant/gemessen } \\
\text { Methanausbeute in \% } \\
\text { Gehalt an } \mathrm{H}_{2} \mathrm{~S}\end{array}$ & $\downarrow$ & $\uparrow$ \\
& $\begin{array}{l}\downarrow \\
\text { BHKW }\end{array}$ & $\downarrow$ & $\uparrow$ \\
\hline
\end{tabular}

Quelle: Eigene Darstellung

Auf der Ebene der Fermentierung sind die Methanausbeute in \% und die erzeugte Menge Biogas in $\mathrm{Nm}^{3}$ pro Jahr entscheidende Kennziffern. Als Leistungsindikatoren könnten die „Abweichung von der geplanten Biogasmenge in $\mathrm{Nm}^{3}$ pro Jahr oder Monat“ und die „Methanausbeute in Prozent" verwendet werden. Diese Kennziffern können leicht gemessen werden, so dass der Kontrollaufwand für die Parameter als 
niedrig eingeschätzt werden kann. Die Methanausbeute wird von verschiedenen Faktoren beeinflusst. Beispielhaft sind der Wassergehalt des Substrates, die Gärtemperatur, die Verweilzeit, die Substrataufbereitung und der Grad des Substrataufschlusses zu nennen (SchatTAUER UND WEILAND 2005: 25 ff.). Da die erzeugte Biogasmenge und der Methangehalt von der Substratqualität abhängen, unterliegen die genannten Parameter Einflüssen, die nicht durch den Anlagenbetreuer verändert werden können. Die komplexen Wechselwirkungen zwischen den verschiedenen chemischen Verbindungen und biologischen Komponenten im Gärgemisch machen die genannten Parameter störanfällig. Dies sei exemplarisch am Schwefelgehalt verdeutlicht. Die Reinheit des erzeugten Biogas hängt u.a. vom Schwefelwasserstoffgehalt des Gärsubstrats ab (ebenda: 31). Ein zu hoher Gehalt an Schwefelwasserstoff hemmt den Abbauprozess und kann zu Korrosionsschäden führen. Schwefel ist für die Methan bildenden Bakterien aber auch ein essentielles Spurenelement und kann in Form von Sulfidionen schädliche Schwermetalle binden und ausfällen (ebenda: 28). An der Biogasbildung sind verschiedene säurebildende Bakterien maßgeblich beteiligt. Der Anteil der Essigsäure- und Methanbildner hat einen starken Einfluss auf die Methanbildung und wird auch durch den Betreuer der Biogasanlage beeinflusst. Die Säurenzusammensetzung des Fermentergemischs wäre zwar einfach zu messen und könnte sich daher als Leistungsindikator anbieten, es gibt jedoch einige Punkte, die dagegen sprechen. Da die Bakterienarten in enger Symbiose miteinander leben, kann das biologische Gleichgewicht im Fermenter durch Eingriffe leicht gestört werden. Außerdem hängt die Bakterienzusammensetzung stark vom Substrat ab. Eine Festlegung der optimalen Zusammensetzung im Vorhinein würde also homogene Substrate voraussetzen. Homogene Substrate werden in der Praxis nicht durchgängig erreichbar sein. Der entsprechend Entlohnte müsste dann auch für die Beschaffung hochqualitativer Substrate verantwortlich sein.

Auf der Ebene des Blockheizkraftwerkes (BHKW) kann die Leistung des Anlagenbetreuers durch den „eingespeisten Strom in $\mathrm{kWh}_{\mathrm{el}}$ pro Jahr“" gemessen werden. Als aggregierter Parameter für die Leistung der Biogasanlage eignet sich dieser Parameter gut, da er durch die oben aufgeführten Kennziffern direkt beeinflusst wird. Es ist zu bedenken, dass die tatsächlich erzielte Leistung der Biogasanlage von den Planwerten in der Anfangszeit stark abweichen kann, da im Regelfall erst Erfahrungen mit der Biogasgewinnung und den Wechselwirkungen zwischen den verschiedenen 
Bakterien und Inhaltstoffen im Fermenter gewonnen werden müssen. Dies kann zu einer starken Volatilität des Einkommens des Anlagenbetreuers führen, wenn dieser anhand der oben genannten Leistungsindikatoren leistungsorientiert entlohnt wird. Nach Untersuchungen der Bundesforschungsanstalt für Landwirtschaft, erreicht ein Drittel der betrachteten 59 Anlagen noch nicht einmal $70 \%$ der elektrischen Auslastung (NEUMANN 2006: 130).

Für die leistungsorientierte Entlohnung für die Betreuer von Biogasanlagen bietet die Zukunft die Perspektive, dass verlässlichere Daten zur Bestimmung der vorgeschlagenen Bemessungsgrundlagen zur Verfügung stehen werden. Die steigende Anzahl an Biogasanlagen wird die Basis für aussagefähige Betriebsvergleiche verbessern. Dadurch wird die Objektivität und Gerechtigkeit leistungsabhängiger Vergütungssysteme verbessert werden.

\subsection{Exemplarische betriebswirtschaftliche Auswirkungen von Leistungslöhnen in verschiedenen Betriebszweigen}

Nach dem für ausgewählte Betriebszweige die Vor- und Nachteile einer Reihe von Leistungsindikatoren erläutert wurde, soll in diesem Abschnitt die Wirkung von Leistungslöhnen auf den Erfolg des Betriebszweigs anhand verschiedener Beispiele veranschaulicht werden. Es werden für die Betriebszweige Ackerbau, Milchproduktion, Sauenhaltung und Biogasproduktion unterschiedliche Situationen dargestellt. Diese beruhen auf den folgenden Annahmen:

Die Ausgangssituation weist ein Produktivitätsniveau von $100 \%$ aus. Dieses wird als die Normalleistung definiert. Die Daten für die Simulation der Ausgangssituation beruhen auf den Durchschnittsbetrieben der Betriebszweigabrechnung unterschiedlicher Beratungsinstitutionen, da keine mehrjährigen Vergleichsdaten vorlagen. Da keine vergleichbaren Betriebszweigabrechnungen für die Biogasproduktion vorlagen, wurde in diesem Betriebszweig für die Simulation der Ausgangssituation der DLGBeispielsbetrieb der „Betriebszweigabrechnung Biogas“ (DLG 2006) zur Hilfe genommen. Für die Praxis lautet aber die Empfehlung, sich für die Festlegung der Normalleistung am mehrjährigen Betriebsdurchschnitt zu orientieren.

Im nächsten Schritt wird die Auswirkung einer Produktivitätssteigerung von $5 \%$ gegenüber der Normalleistung auf den Gewinn des Betriebszweigs simuliert. Die 
Produktivitätssteigerung soll durch ein Leistungslohnsystem bewirkt werden. Die Höhe der Produktivitätssteigerung ist hypothetisch, da es keine empirischen Daten darüber gibt, wie stark sich ein Anreizsystem auf die Produktivität in der Landwirtschaft auswirkt.

Als Parameter wird ein einfach zu messender Leistungsindikator zugrunde gelegt. Da die verwendeten Leistungsindikatoren in den Betriebszweigen ohnehin gemessen werden, wird angenommen, dass in diesem Fall die Transaktionskosten für die Kontrolle der tatsächlich erreichten Leistung zu vernachlässigen sind.

Es wird ferner angenommen, dass den Mitarbeitern eine Prämie in Höhe von $10 \%$ eines festen Grundlohns gewährt wird, wenn sie eine Produktivitätssteigerung von $5 \%$ erreichen. Es werden keine Abzüge für Leistungen unterhalb des Normalwerts (100\%) vorgenommen, da diese aufgrund der grundsätzlichen Störanfälligkeit biologischer Produktivitätskennziffern zu einem starken Motivationseinbruch führen können. Die Prämie ist mit $10 \%$ des Ausgangsgehalts niedrig angesetzt, wenn man berücksichtigt, dass motivationstheoretisch erst Prämien ab $10 \%$ des Gesamtlohns verhaltenswirksam werden (vgl. hierzu die Empfehlungen der SÄCHSISCHEN LANDESANSTALT FÜR LANDWIRTSCHAFT 2002: 39).

Die dritte dargestellte Situation ist das Produktivitätsniveau von $95 \%$. In diesem Fall erhalten die Mitarbeiter nur ihren Grundlohn. Dieses Produktivitätsniveau könnte zum Beispiel eintreten, wenn durch das Leistungslohnsystem ein Motivationsrückgang induziert wird, falls beispielsweise das Vergütungssystem von den Mitarbeitern als ungerecht empfunden wird oder intrinsische Motivation verdrängt wird.

Es werden alle Kosten außer den Personalkosten unabhängig vom simulierten Outputniveau konstant gehalten, da es keine Veränderungen im Produktionsverfahren gibt. Inwieweit diese Annahme zutreffend ist, wird bei der Analyse der unterschiedlichen Betriebszweige diskutiert. 


\subsubsection{Exemplarische betriebswirtschaftliche Auswirkungen eines Leistungslohnsystems im Ackerbau}

Als erstes Beispiel soll eine Leistungssteigerung bei einem Mitarbeiter im Betriebszweig Ackerbau simuliert werden, die sich in einer Steigerung des Hektarertrages (in Getreideeinheiten) um $5 \%$ gegenüber dem vierjährigen Betriebsdurchschnitt ausdrückt. Die Ausgangsdaten liefert der Durchschnitt der Marktfruchtbetriebe der LANDWIRTSCHAFTSKAMMER SCHLESWIG-HOLSTEIN für den Zeitraum des Wirtschaftsjahres 2003-2004 (2005: 8). Wie bereits erwähnt, soll die Produktivitätssteigerung durch eine Prämie in Höhe von $10 \%$ des Lohnes bei Normalleistung induziert werden.

Das Naturalertragsniveau in der Ausgangssituation beträgt $81,74 \mathrm{dt}$ GE/ha (LANDWIRTSCHAFTSKAMMER SCHLESWIG-HolsteIn 2005b: 12). Im Durchschnittsbetrieb wurde eine Marktleistung von 1.113,36 Euro/ha erreicht. Eine Dezitonne Getreideeinheit hatte damit einen Wert von 13,62 Euro. Die Summe der Leistungen insgesamt wird in der Ist-Situation mit 1.605 Euro ausgewiesen. Die Differenz zwischen der Marktleistung und der Summe der Leistungen des Betriebszweigs ergibt sich aus den Direktzahlungen und den sonstigen Leistungen. Die Direktzahlungen sind in der Betriebszweigabrechnung der Landwirtschaftskammer Schleswig-Holstein nicht ausgewiesen. Sie wurden aus den Angaben über die durchschnittliche Fruchtfolge (ebenda: 6) ${ }^{28}$ und den im Jahr 2003 in Schleswig-Holstein bezahlten Preisausgleichszahlungen der EU berechnet (MINISTERIUM FÜR LANDWIRTSCHAFT, UMWELT UND LÄNDLICHE RÄUME DES LANDES SCHLESWIGHOLSTEIN 2007).

\footnotetext{
28 Neben den dominierenden Früchten Winterweizen, Winterraps und Wintergerste wurden im WJ 2003/2004 in Schleswig-Holstein durchschnittlich 5,2 \% der Ackerfläche mit sonstigen Früchten angebaut. Hierzu gehören z.B. Roggen und Zuckerrüben. Es wird angenommen, dass 2,5\% der Ackerfläche mit Roggen oder Triticale oder bestellt waren, für die die Getreideprämie von 429,03 Euro gewährt wurde.
} 
TABELLE 37: KOSTEN UND NUTZEN DES LEISTUNGSLOHNSYSTEMS IM ACKERBAU

Leistungsniveau

\begin{tabular}{|c|c|c|c|c|}
\hline & \\
\hline & & $105 \%$ & $100 \%$ & $95 \%$ \\
\hline & GE/ha & 85,82 & 81,74 & 77,65 \\
\hline & Preis/GE & 13,62 & 13,62 & 13,62 \\
\hline Marktleistungen & $€ /$ ha & $1.169,03$ & $1.113,36$ & $1.057,69$ \\
\hline Direktzahlungen & $€ /$ ha & 430,64 & 430,64 & 430,64 \\
\hline sonstige Leistungen & $€ /$ ha & 61,00 & 61,00 & 61,00 \\
\hline Summe Leistungen & $€ /$ ha & $1.660,67$ & $1.605,00$ & $1.549,33$ \\
\hline Direktkosten & $€ /$ ha & 384,00 & 384,00 & 384,00 \\
\hline Lohnkosten & $€ /$ ha & 212,30 & 193,00 & 193,00 \\
\hline sonstige Arbeitserledigungskosten & $€ /$ ha & 396,00 & 396,00 & 396,00 \\
\hline Summe Arbeitserledigungskosten & $€ /$ ha & 608,30 & 589,00 & 589,00 \\
\hline Gebäude-, Flächen- und sonstige Kosten & $€ /$ ha & 104,00 & 104,00 & 104,00 \\
\hline Reinertrag pro Hektar & $€ /$ ha & 564,37 & 528,00 & 472,33 \\
\hline Lohnkostendifferenz des Leistungslohnsystems & $€ /$ ha & 19,30 & 0 & 0 \\
\hline $\begin{array}{l}\text { Indirekte Kosten des Leistungslohnsystems } \\
\text { (Verdrängungseffekt) }\end{array}$ & $€ /$ ha & 0 & 0 & $-55,67$ \\
\hline Nutzen des Leistungslohnsystems & $€ /$ ha & 55,67 & 0 & 0 \\
\hline Gesamteffekt des Leistungslohnsystems in Euro/ha & $€ /$ ha & 36,37 & 0,00 & $-55,67$ \\
\hline Effizienz des Leistungslohnsystems bei $105 \%$ Leistung & 1:x & 2,88 & 0 & 0 \\
\hline
\end{tabular}

Quelle: Eigene Berechnungen, Ist-Situation basierend in Anlehnung an Daten der LANDWIRTSCHAFTSKAMMER SCHLESWIG-HOLSTEIN (2005b: 6 ff.).

Die Summe der Direkt-, Arbeits- und sonstigen Kosten wird in den verschiedenen simulierten Produktivitätsniveaus aus Vereinfachungsgründen konstant gehalten, da sich das Produktionssystem durch das Leistungslohnsystem nicht ändern soll, sondern lediglich die Arbeitsleistung des/der Mitarbeiter variiert wird. Zwar gilt in der Landwirtschaft das Prinzip des abnehmenden Grenzertrags, die Auswertungen der Landwirtschaftskammer Schleswig-Holstein zeigen aber, das sich das obere Quartil der ausgewerteten Betriebe von den mittleren $50 \%$ der Betriebe nur um 5 Euro/ha unterscheiden. Da das untere Quartil eine deutlich ungünstigere Kostenstruktur als die mittlere und beste Erfolgsgruppe aufweist, kann nicht abgeleitet werden, dass steigende Erträge zu höherem Input führen. Es zeigt sich sogar, dass die oberen 25 \% der Betriebe mit geringeren Direktkosten und Abschreibungen bewirtschaftet werden 
(LANDWIRTSCHAFTSKAMMER SCHLESWIG-HOLSTEIN 2005b: 10). Offenbar drückt ein steigendes Ertragsniveau eine effizientere Wirtschaftsweise aus.

Durch das Leistungslohnsystem steigen die Lohnkosten um 19,30 Euro pro Hektar, wenn die vorgesehene Produktivitätssteigerung erreicht wird. Der steigende Naturalertrag führt bei der in Tabelle 37 ausgewiesenen Preiskonstellation zu einem Nutzen des Vergütungssystems von 55,67 Euro. Der Saldo aus Nutzen und Kosten beträgt 36,37 Euro pro Hektar. Die Durchschnittsproduktivität der Leistungsentlohnung beträgt 1:2,88. Durchschnittlich wird also mit einem Euro Prämie der Gewinn des Betriebszweigs um 2,88 Euro gesteigert. Wie in Kapitel 3.3.2 ausgeführt wurde, kann ein Leistungslohnsystem auch zum Motivationsrückgang führen. Wenn dieser Fall eintreten und zu einem hypothetischen Produktivitätsrückgang von $5 \%$ gegenüber der Normalleistung führen würde, würde der Gewinn des Betriebszweigs Ackerbau um 55,67 Euro zurückgehen.

\subsubsection{Exemplarische betriebswirtschaftliche Auswirkungen eines Leistungslohnsystems in der Milchproduktion}

Im Folgenden wird gezeigt, wie sich eine Produktivitätssteigerung von $5 \%$ in der durchschnittlichen Milchleistung - gemessen in $\mathrm{kg}$ pro Kuh und Jahr - auf die Wirtschaftlichkeit des Betriebszweigs (in Euro pro Kuh) auswirkt. Die Produktivitätssteigerung soll durch eine Prämie von $10 \%$ des Gehalts induziert werden, die bei der Erfüllung der geplanten Milchleistungssteigerung von $5 \%$ gewährt wird.

Als Ist-Situation wird der Durchschnittsbetrieb der Betriebszweigabrechnung Rind der Landwirtschaftskammer Schleswig-Holstein für das Wirtschaftsjahr 2003/2004 zugrunde gelegt (vgl. Tabelle 38). In diesem Betrieb werden durchschnittlich 80 Milchkühe mit einer Milchleistung von $7.972 \mathrm{~kg}$ gehalten. Quotenkosten durch den Anstieg der Milchleistung sollen in dem Szenario nicht berücksichtigt werden. Analog $\mathrm{zu}$ den Berechnungen für den Betriebszweig Ackerbau werden ceteris paribus alle Kosten außer den Löhnen konstant gehalten und auf der Ertragsseite alle Leistungen außer dem Milcherlösen unverändert gelassen. Intuitiv erscheint diese Vorgehensweise als zu einfach, da mit steigender Milchleistung auch der Input steigen müsste. Die Auswertungen der Landwirtschaftskammer Schleswig-Holstein zeigen aber, dass mit steigender Milchleistung die Direktkosten pro kg erzeugter Milch sinken und dadurch die Direktkosten insgesamt pro Kuh trotz der höheren Milchleistung nicht höher als in 
der Ausgangssituation sind (LANDWIRTSCHAFTSKAMMER SCHLESWIG-HolsteIn 2005a: 12). Zwar steigen die Kraftfutterkosten pro kg erzeugte Milch an, dafür sinken aber die Grundfutterkosten, da Grundfutter effizienter eingesetzt wird. Auch die Arbeitserledigungs- und Gemeinkosten sinken pro kg Milch.

TABELLE 38: KoSTEN UND NUTZEN DES LEISTUNGSLOHNSYSTEMS IN DER MILCHPRODUKTION

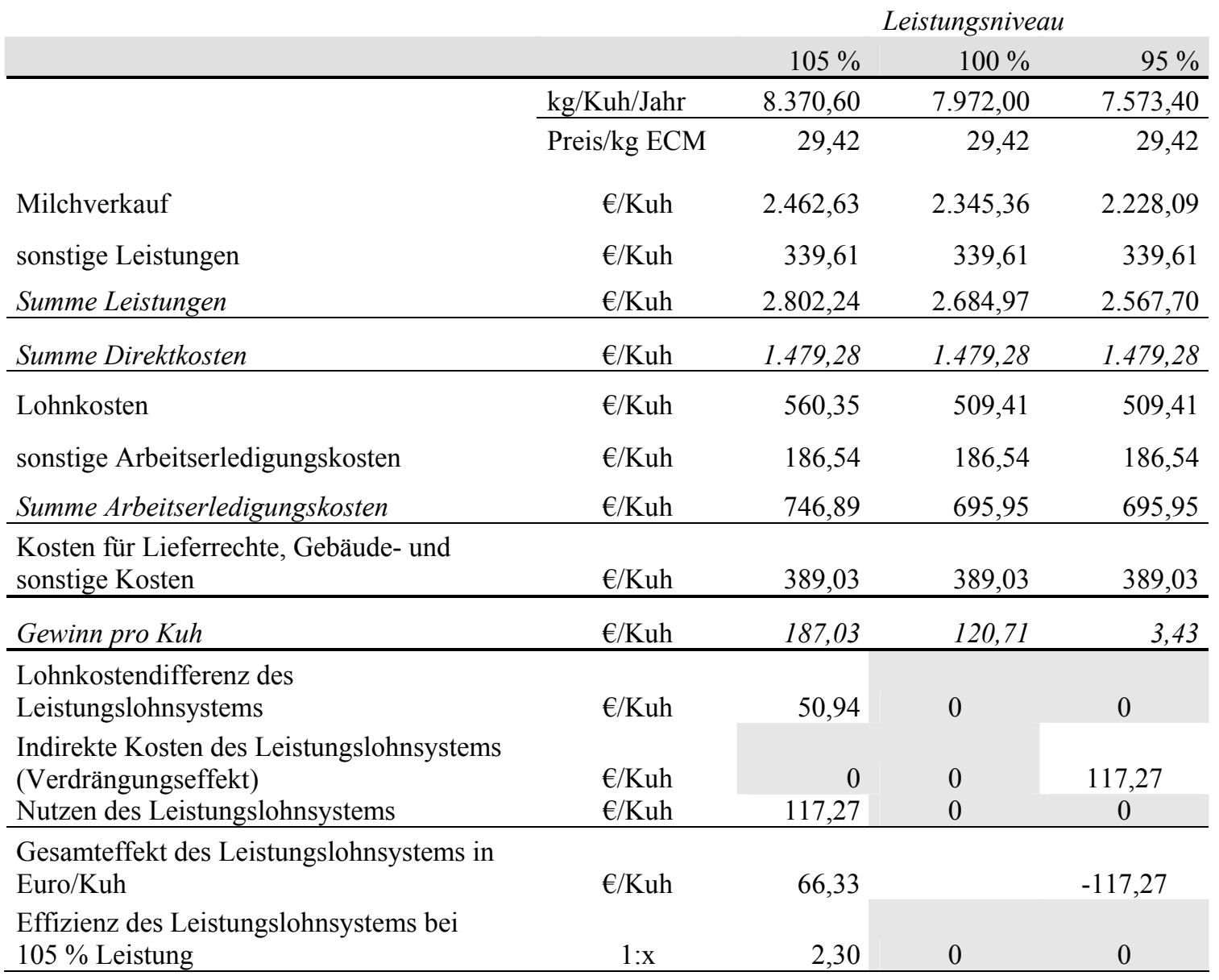

Quelle: Eigene Berechnungen, Ist-Situation basierend auf Daten der LANDWIRTSCHAFTSKAMMER SCHLESWIG-HOLSTEIN 2005a: 12

Steigt die Produktivität des Betriebszweigs um $5 \%$ durch das Leistungslohnsystem, dann wären für die Bezahlung der Leistungsprämie 50,94 Euro pro Kuh aufzuwenden. Die Milchleistungssteigerung würde dem Betriebszweig einen zusätzlichen Erlös von 117,27 Euro pro Kuh einbringen. Abzüglich des Mehraufwands an Personalkosten würde sich ein Gesamteffekt von 66,33 Euro pro Kuh ergeben. Die Durchschnittsproduktivität des Leistungslohnsystems beträgt in diesem Fall 1:2,30. Sie wäre damit etwas geringer als im Ackerbau. Dies liegt an der Arbeitsintensität der Milchproduktion und den hohen Lohnkostenbelastungen des Betriebszweigs. Die Lohnkosten haben im oben aufgeführten Beispielsbetrieb einen Kostenanteil von $20 \%$ 
in der Ausgangssituation, der durch das Leistungslohnssystem auf $21 \%$ steigt. Im Ackerbau hatten die Lohnkosten einen Anteil von $18 \%$ in der Ausgangssituation.

\subsubsection{Exemplarische betriebswirtschaftliche Auswirkungen eines Leistungslohnsystems in der Sauenhaltung}

Der Einfluss der Mitarbeiter auf biologische Leistungskennzahlen ist in der Sauenhaltung weitaus größer als in der Schweinemast. Die Auswirkung produktionstechnischer Unterschiede wird in der Schweinemast stark von den Entwicklungen an den In- und Outputmärkten überlagert (ebenda: 73). Hinzukommt, dass z.B. die Schweinemäster im Kammergebiet Schleswig-Holstein biologische Kennziffern (z.B. der Magerfleischanteil, die täglichen Zunahmen) erreichen, die innerhalb der letzten Wirtschaftjahren nicht mehr variieren, also ein Plateau erreicht haben (LANDWIRTSCHAFTSKAMMER SCHLESWIG-HolSTEIN 2007). In diesen Fällen sind weiteren Bemühungen zur Verbesserung der genannten Kennzahlen aus biologischzüchterische Grenzen gesetzt. Aus diesen Gründen wird im Folgenden die Gewährung einer Prämie auf die Erhöhung der Kennzahl aufgezogene Ferkel pro Sau und Jahr und die betriebswirtschaftlichen Auswirkungen dieses Prämienlohnsystems diskutiert.

Die Kalkulationen basieren auf Betriebszweigauswertungen der SÄCHSISCHEN LANDESANSTALT FÜR LANDWIRTSCHAFT (2005) für das Wirtschaftsjahr 2003/2004. Die für den Erhalt der Prämie notwendige Leistungssteigerung soll $5 \%$ betragen. Dies entspricht bei einer Normalleistung von 21,4 aufgezogenen Ferkeln pro Sau und Jahr einer Steigerung auf 22,47 Ferkel pro Sau und Jahr. Wieder soll der Leistungsanreiz durch die Auszahlung einer Prämie äquivalent $\mathrm{zu}$ einer zehnprozentigen Gehaltserhöhung gewährt werden.

Außer den Lohnkosten werden alle weiteren Kosten konstant gehalten, da sich das Produktionssystem nicht ändern soll. Die Auswertungen der LANDWIRTSCHAFTSKAMMER SCHLESWIG-HOLSTEIN (2005a: 67, 2007: 8) zeigen, dass sich die erfolgreichen von den durchschnittlichen Betrieben in der Höhe der Direktkosten nur geringfügig unterscheiden. Die sonstigen Arbeitserledigungskosten, Gebäude- und weiteren Gemeinkosten sind in der Betriebszweigabrechnung der Landwirtschaftskammer Schleswig-Holstein nicht ausgewiesen. Die Auswertung der Sächsischen Landesanstalt für Landwirtschaft für das Wirtschaftsjahr 2003/2004 umfasst leider nur sechs Sauenanlagen mit eigener Reproduktion. In den besseren 
Betrieben wird eine höhere Leistung mit geringeren Direktkosten, etwas höheren Arbeitserledigungskosten, deutlich geringeren Gebäude- und sonstigen Kosten erzielt. Aus den vorliegenden Auswertungen lässt sich ableiten, dass noch deutliche Reserven im Herdenmanagement liegen, die eine Produktivitätssteigerung auch ohne höheren Input oder sogar mit Kosteneinsparungen möglich machen.

Die Tabelle 39 zeigt die Auswirkungen des Leistungslohnsystems unter den oben definierten Annahmen.

TABELLE 39: KOSTEN UND NUTZEN DES LEISTUNGSLOHNSYSTEMS IN DER FERKELPRODUKTION

\begin{tabular}{|c|c|c|c|c|}
\hline & & \multicolumn{3}{|c|}{ Leistungsniveau } \\
\hline & & $105 \%$ & $100 \%$ & $95 \%$ \\
\hline & Ferkel/Sau/Jahr & 22,47 & 21,40 & 20,33 \\
\hline & Preis/Ferkel & 39,58 & 39,58 & 39,58 \\
\hline Ferkelverkauf & $€ /$ Sau & 889,36 & 847,01 & 804,66 \\
\hline sonstige Leistungen & $€ /$ Sau & 157,57 & 157,57 & 157,57 \\
\hline Summe Leistungen & $€ /$ Sau & 1046,93 & 1004,582 & 962,23 \\
\hline Direktkosten & $€ /$ Sau & 701,03 & 701,03 & 701,03 \\
\hline Lohnkosten & $€ /$ Sau & 195,95 & 178,14 & 178,14 \\
\hline sonstige Arbeitserledigungskosten & $€ /$ Sau & 71,77 & 71,77 & 71,77 \\
\hline Summe Arbeitserledigungskosten & $€ /$ Sau & 267,72 & 249,91 & 249,91 \\
\hline Gebäude-, und sonstige Kosten & $€ /$ Sau & 130,93 & 130,93 & 130,93 \\
\hline Gewinn pro Sau & $€ /$ Sau & $-52,75$ & $-77,29$ & $-119,64$ \\
\hline $\begin{array}{l}\text { Lohnkostendifferenz des } \\
\text { Leistungslohnsystems } \\
\text { Indirekte Kosten des } \\
\text { Leistungslohnsystems } \\
\text { (Verdrängungseffekt) }\end{array}$ & $€ /$ Sau & 17,81 & & $-42,35$ \\
\hline Nutzen des Leistungslohnsystems & $€ /$ Sau & 42,35 & 0 & 0 \\
\hline $\begin{array}{l}\text { Gesamteffekt des Leistungslohnsystems } \\
\text { in Euro/Sau }\end{array}$ & $€ /$ Sau & 24,54 & 0,00 & $-42,35$ \\
\hline $\begin{array}{l}\text { Effizienz des Leistungslohnsystems bei } \\
105 \% \text { Leistung }\end{array}$ & $1: \mathrm{x}$ & 2,38 & & \\
\hline
\end{tabular}

Quelle: Eigene Berechnungen, Ist-Situation basierend auf Daten der SÄCHSISCHEN LANDESANSTALT FÜR LANDWIRTSCHAFT 2005: 42

Es zeigt sich, dass durch das Vergütungssystem ein positiver wirtschaftlicher Effekt von 24,53 Euro pro Sau erreicht werden kann. Dieser Gesamteffekt des Vergütungssystems setzt sich aus den gegenüber der Normalleistung erhöhten Lohnkosten von 17,81 Euro 
und einer Umsatzerhöhung von 42,35 Euro zusammen. Pro eingesetzten Euro würde sich bei einer Steigerung der Produktivität um $5 \%$ die Wirtschaftlichkeit der Sauenhaltung um 2,38 Euro verbessern. Bei einem Produktivitätsrückgang um $5 \%$ würde der Gesamteffekt bei -42,35 Euro liegen.

\subsubsection{Exemplarische betriebswirtschaftliche Auswirkungen eines Leistungslohnsystems für Biogasanlagen}

Der DLG-Beispielbetrieb, auf dessen Betriebszweigabrechnung die folgende Kalkulation basiert, ist eine GbR, die eine 190 kW Biogasanlage betreibt. Die Substrate werden von den Gesellschaftern gekauft. Die Anlage wird von einem Beschäftigten und einem Gesellschafter geleitet. Im DLG-Beispiel wird angenommen, dass die Biogasanlage $89 \%$ der Nennleistung erreicht. Die Simulation startet also bereits auf einem sehr hohen Outputniveau. Die Marktleistung errechnet sich aus der Vergütung für den eingespeisten Strom, bestehend aus Grundvergütung, NAWARO- und KWKBonus sowie aus dem Verkauf der erzeugten Abwärme an einen Ferkelerzeuger (DLG 2006: 39-43).

In Abweichung vom DLG-Beispiel werden in den Kalkulationen der Personalaufwand und der Lohnansatz für den mitarbeitenden Gesellschafter zusammengefasst. Es wird angenommen, dass es sich um eine Lohnarbeitskraft handelt, die die für die Betreibung der Biogasanlage notwendigen 1.500 Arbeitsstunden erbringt. Wie auch in den zuvor aufgeführten Beispielen soll der Motivationsanreiz durch eine Prämie in Höhe von 10 \% des Lohnes hervorgerufen werden, die bei einer Steigerung von $5 \%$ der Leistung gemessen in der Menge eingespeisten Stroms in $\mathrm{kWh}_{\mathrm{el}}$ - gewährt wird. Analog zu den zuvor dargestellten Simulationen werden nur das Outputniveau und die Personalkosten variiert, alle weiteren Kosten werden konstant gehalten. 
TABELLE 40: KOSTEN UND NUTZEN DES LEISTUNGSLOHNSYSTEMS IN DER BIOGASPRODUKTION

\begin{tabular}{|c|c|c|c|c|}
\hline & & \multicolumn{3}{|c|}{ Leistungsniveau } \\
\hline & & $105 \%$ & $100 \%$ & $95 \%$ \\
\hline & $\mathrm{kwh}_{\mathrm{el}}$ & 1.537 .906 & 1.464 .672 & 1.391 .438 \\
\hline & $\begin{array}{l}\text { Preis (inkl. Boni } \\
\text { in Cent) } / \mathrm{kwh}_{\mathrm{el}}\end{array}$ & 18,17 & 18,17 & 18,17 \\
\hline Stromverkauf, Boni & $€ /$ Anlage & $279.437,45$ & $266.130,90$ & $252.824,36$ \\
\hline sonstige Leistungen & $€ /$ Anlage & $17.246,56$ & $17.246,56$ & $17.246,56$ \\
\hline Summe Leistungen & $€ /$ Anlage & $296.684,01$ & $283.377,46$ & $270.070,92$ \\
\hline Summe Direktkosten & $€ /$ Anlage & $109.088,09$ & $109.088,09$ & $109.088,09$ \\
\hline Summe Anlagenkosten & $€ /$ Anlage & $45.882,45$ & $45.882,45$ & $45.882,45$ \\
\hline Lohnkosten & $€ /$ Anlage & $21.175,00$ & $19.250,00$ & $19.250,00$ \\
\hline Zündöl und Schmierkosten & $€ /$ Anlage & $14.207,32$ & $14.207,32$ & $14.207,32$ \\
\hline Stromzukauf & $€ /$ Anlage & $17.136,66$ & $17.136,66$ & $17.136,66$ \\
\hline sonstige Betriebskosten & $€ /$ Anlage & $8.519,00$ & $8.519,00$ & $8.519,00$ \\
\hline Summe Betriebskosten & $€ /$ Anlage & $61.037,98$ & $59.112,98$ & $59.112,98$ \\
\hline $\begin{array}{l}\text { Summe Gebäude- und } \\
\text { Grundstückskosten }\end{array}$ & $€ /$ Anlage & $26.399,22$ & $26.399,22$ & $26.399,22$ \\
\hline $\begin{array}{l}\text { Kosten für Maschinen und sonstige } \\
\text { Kosten }\end{array}$ & $€ /$ Anlage & 9188,77 & 9188,77 & 9188,77 \\
\hline Gewinn pro Anlage & $€ /$ Anlage & $54.276,27$ & $42.894,72$ & $29.588,18$ \\
\hline $\begin{array}{l}\text { Lohnkostendifferenz des } \\
\text { Leistungslohnsystems }\end{array}$ & $€ /$ Anlage & $1.925,00$ & 0 & 0 \\
\hline $\begin{array}{l}\text { Indirekte Kosten des } \\
\text { Leistungslohnsystems } \\
\text { (Verdrängungseffekt) }\end{array}$ & $€ /$ Anlage & 0 & 0 & $-13.306,55$ \\
\hline $\begin{array}{l}\text { Nutzen des Leistungslohnsystems } \\
\text { Gesamteffekt des }\end{array}$ & $€ /$ Anlage & $13.306,55$ & 0 & 0 \\
\hline $\begin{array}{l}\text { Leistungslohnsystems in } \\
\text { Euro/Anlage }\end{array}$ & $€ /$ Anlage & $11.381,55$ & 0,00 & $-13.306,55$ \\
\hline $\begin{array}{l}\text { Effizienz des } \\
\text { Leistungslohnsystems bei } 105 \%\end{array}$ & & & & \\
\hline Leistung & $1: \mathrm{x}$ & 6,91 & 0 & 0 \\
\hline
\end{tabular}

Quelle: Eigene Berechnungen, Ist-Situation basierend auf Daten der DLG 2006: 41

Durch das Leistungslohnsystem ergeben sich bei der vorgesehenen Produktivitätssteigerung zusätzliche Lohnkosten von 1.925 Euro pro Jahr. Es ergäbe ich ein zusätzlicher Nutzen von 13.306,55 Euro durch die Mehrlieferung an elektrischer Energie. Der Wärmeverkauf der Anlage würde konstant bleiben, da im DLGBeispielsbetrieb auch in der Ausgangssituation nur ein Teil der Abwärme verkauft werden kann. Bei einer Produktivitätssteigerung von $5 \%$ würde der Gesamteffekt des Vergütungssystem 11.381,55 Euro betragen. Bei einem Produktivitätsrückgang um 5 \% wäre ein negativer Effekt von -13.306,55 Euro $\mathrm{zu}$ erwarten. Die Durchschnittsproduktivität der Vergütungssystems würde im günstigen Fall 1:6,91 betragen. Da die Lohnkosten nur einen Anteil von rund $8 \%$ an den Gesamtkosten 
haben, würde die Wirtschaftlichkeit des Betriebszweiges durch das Vergütungssystem stark steigen, sofern kein Verdrängungseffekt auftritt.

Abschließend ist zu sagen, dass die Festsetzung der Normleistung auf $89 \%$ der Nennleistung der Biogasanlage sehr hoch gegriffen ist und exemplarisch zu sehen ist. In vielen Biogasanlagen werden $89 \%$ der Nennleistung - besonders in der Anfangsphase nicht erreicht. Es empfiehlt sich daher, zunächst verlässliche Daten über das tatsächlich erreichbare Leistungsniveau in der Biogasanlage zu ermitteln, bevor ein leistungsorientiertes Vergütungssystem eingeführt wird.

\subsection{Perspektiven der leistungsorientierten Entlohnung in der Landwirtschaft}

Als Fazit aus den Kalkulationen zur Wirkung von Leistungslöhnen lässt sich festhalten, dass durch das Prämienlohnsystem die Wirtschaftlichkeit der betrachteten Betriebszweige steigen würde, wenn sich die gewünschte Anreizwirkung einstellt. Zwar nehmen die Personalkosten insgesamt zu, dies wird aber durch den Mehrertrag in allen Betriebszweigen kompensiert. Die Effizienz des Vergütungssystems hängt vom Anteil der Lohnkosten an den Gesamtkosten und der tatsächlich erreichten Produktivitätssteigerung ab. Die angeführten Beispiele haben einen rein exemplarischen Charakter, da die potentielle Produktivitätssteigerung vom jeweiligen Ausgangsniveau abhängt. In Betrieben mit bereits sehr hohen biologischen oder technologischen Leistungen ist eine weitere Steigerung um $5 \%$ weniger realistisch als in Betrieben mit unterdurchschnittlichen Leistungen. Ferner hängt es vom Mitarbeiter ab, welche Wirkung das Vergütungssystem hat. So lässt sich festhalten, dass die Vorteile des Vergütungssystems eher in Betrieben mit einer wenig intrinsisch motivierten Belegschaft und durchschnittlichen bis unterdurchschnittlichen biologischen bzw. technologischen Leistungen eher zum Tragen kommen.

Es zeigt sich aber auch, dass Leistungslöhne im Vergleich zu immateriellen Anreizsystemen ein teures Motivationsinstrument sind, wenn die gleiche Leistungssteigerung möglicherweise durch andere Maßnahmen erreicht werden könnte.

Die sich für die leistungsorientierte Entlohnung in der Landwirtschaft ergebenden Perspektiven müssen sich an den zuvor genannten Rahmenbedingungen messen lassen. Die Befragung hat gezeigt, dass Leistungslöhne in der Landwirtschaft derzeit vor allem 
von Betrieben mit mehreren Lohnarbeitskräften und bevorzugt in der Tierproduktion eingesetzt werden. Mit dem fortschreitenden Strukturwandel wird die Bedeutung des Personalmanagements weiter zunehmen und Betriebsleiter werden sich vermehrt der Frage einer optimalen Gestaltung von Anreizsystemen widmen.

Die Motivationswirkung von Leistungslöhnen beruht auf der Einhaltung verschiedener Erfolgbedingungen. Einige dieser Erfolgsbedingungen liegen heute und auch zukünftig in der Hand des Betriebsleiters, dies betrifft insbesondere die Festlegung und Kommunikation erreichbarer Zielgrößen als Bemessungsgrundlage. Ein steigendes Angebot an Unternehmerschulungen im Bereich der so genannten „soft skills“ lässt auf eine steigende Kompetenz der Betriebsleiter hoffen.

Es ist davon auszugehen, dass sich die Persönlichkeitsstruktur der Mitarbeiter in der Landwirtschaft in Zukunft kaum ändern wird. Das attestierte hohe Maß an intrinsischer Motivation bei den Mitarbeitern in der Landwirtschaft wird wohl auch zukünftig bestehen bleiben. Denn vergleichsweise lange und unregelmäßige Arbeitszeiten und ein niedriges Lohnniveau werden auch mittel- bis langfristig Charakteristika des Arbeitsplatzes in der Landwirtschaft bleiben. Es werden sich folglich vor allem die Menschen entscheiden in der Landwirtschaft zu arbeiten, die die Vorteile dieses Berufs wie Flexibilität und ein Arbeiten in der Natur schätzen. Einschränkend ist aber anzumerken, dass die befragten Mitarbeiter nicht unbedingt repräsentativ für die Grundgesamtheit der in der Landwirtschaft beschäftigten Lohnarbeitskräfte sind. Weitere Arbeiten sollten eine repräsentativere Stichprobe anstreben, um mehr Rückschlüsse auf die Grundgesamtheit ziehen zu können.

Cross-Compliance-Auflagen und ein steigendes technisches Niveau der landwirtschaftlichen Maschinen machen es zukünftig immer notwendiger, dass die eingesetzten Arbeitskräfte über eine gute Fachausbildung verfügen. Es ist davon auszugehen, dass gerade diese Fachkräfte einen hohen Anspruch an die Führungsqualität ihres Vorgesetzten haben und eine entsprechende Motivation für ihre Tätigkeit mitbringen, da sie sonst alternativ einen Arbeitsplatz außerhalb der Landwirtschaft gewählt hätten.

Die in Kapitel 3 dargestellten theoretischen Ansätze unterschieden sich hinsichtlich des Menschenbildes und den Empfehlungen zur optimalen Gestaltung von Anreizsystemen. Eines ist ihnen jedoch gemeinsam: Leistungslöhne müssen auf einer kardinal 
messbaren, von den Beteiligten beobachtbaren Bemessungsgrundlage fußen, damit sie die gewünschte Leistungssteigerung bewirken. In diesem Punkt bietet die Landwirtschaft mit ihren Besonderheiten keine ideale Voraussetzung für Leistungslohnsysteme. Vor allem in der Pflanzenproduktion ist es schwierig, eine Bemessungsgrundlage zu finden, die den genannten Bedingungen entspricht.

Es ist unzweifelhaft, dass die Kontrollmöglichkeiten durch die zunehmende Bedeutung des Precision Farming erweitert werden und der Kontrollaufwand für die unterschiedlichen Produktionsschritte sinken wird. Dies würde die Verbreitung leistungsorientierter Entlohnungssysteme begünstigen. Die Möglichkeiten des Precision Farming in Bezug auf die Überwachung der Arbeitsabläufe werden von den befragten Mitarbeitern jedoch kritisch gesehen. Sie sprachen in diesem Zusammenhang vom „gläsernen Mitarbeiter“, dessen Handlungsspielräume bei Ausnutzung aller technischen Möglichkeiten eingeschränkt würden. Hier deutet sich eine zukünftige Ursache für Motivationsrückgänge an. Die befragten Mitarbeiter verbinden ihren Arbeitsplatz bisher mit abwechslungsreichen und selbstbestimmten Aufgaben, was zu hoher intrinsischer Motivation führt. Dieses Ergebnis deckt sich auch mit den in Kapitel 2.1 vorgestellten Ausführungen zum Flow-Erlebnis in der Landwirtschaft. Durch den Einsatz modernster Technik könnte sich dieses Erleben des Arbeitsplatzes ändern, wenn diese zur Kontrolle der Mitarbeiter eingesetzt würde. Eine steigende Wahrnehmung von Fremdkontrolle durch die Mitarbeiter, diese steht hinter dem Bild des „gläsernen Mitarbeiters“, kann intrinsische Motivation verdrängen, wie in Kapitel 3.3.2 deutlich wurde. Man könnte die Aussage der betreffenden Mitarbeiter im Ergebnisworkshop auch als potentielle Technikablehnung älterer Schlepperfahrer interpretieren. Es zeigte sich aber in den Diskussionen eine große Begeisterung für technische Innovationen, und die Personen, die Diskussion um den Einsatz des Precision Farming dominierten, waren junge Mitarbeiter.

Als Alternativen zu leistungsabhängigen Vergütungsregelungen, die den Besonderheiten der Landwirtschaft möglicherweise besser gerecht werden und mit geringen Transaktionskosten behaftet sind, bieten sich Effizienzlohnsysteme und Turnierentlohnungen an.

Die Gewährung einer im Vergleich zu anderen landwirtschaftlichen Betrieben überdurchschnittlichen Belohnung ist ein einfaches Mittel, um fähige Mitarbeiter 
anzuziehen und zu halten. Zwar besteht kein unmittelbarer Zusammenhang zwischen Leistung und Lohn, durch die steigenden Kosten eines möglichen Arbeitsplatzverlusts ist aber vor allem im Niedriglohnsektor Landwirtschaft mit einer positiven Motivationsund Selektionswirkung zu rechnen. Zwar sind die befragten Mitarbeiter intrinsisch motiviert, sie verwiesen jedoch in den Diskussionen immer wieder auf die nach ihrer Meinung unadäquate Lohnhöhe.

Turnierentlohnungen als eine Form der relativen Leistungsbewertungen könnten in der Landwirtschaft sowohl auf Produktions- als auch auf Führungsebene eingesetzt werden. Sie eignen sich besonders dann, wenn die kardinale Leistungsbemessung an ihre Grenzen gerät. Da sie negative Auswirkungen auf das Teamverhalten hat und in der Landwirtschaft verschiedene Mitarbeiter in den Produktionsprozess eingreifen, ist es nicht empfehlenswert, die Mitarbeiter eines Betriebes gegeneinander in den Wettbewerb zu bringen. Es wäre aber denkbar, Zusatzprämien für eine relative Mehrleistung im Vergleich zu ähnlichen Betrieben zu gewähren. Betriebsvergleiche legen die Stärken und Schwächen von Betrieben mit unterschiedlicher Betriebsstruktur offen und können als Grundlage zur Leistungsbemessung dienen. Werden die Leistungslöhne anhand der Entwicklung des Leistungsparameters (z.B. der Hektarerträge) im Vergleich zu ähnlichen Betrieben bemessen, so hat dies den Vorteil, dass der Einfluss stochastischer Größen, wie der Witterung, gemindert wird. Das Entlohnungsschema wird dadurch gerechter und die Mitarbeiter können leichter den Zusammenhang zwischen Anstrengung und Leistung herstellen, da es z.B. um schlechte Niederschläge im Erntejahr bereinigt wird. Wichtig ist es aber, den Mitarbeiter die Betriebsvergleiche zu zeigen, damit deutlich wird, auf welchen erzielten Parametern die Leistungsentlohnung beruht.

Die Ausführungen zeigen, dass auch unter dem Einsatz modernsten technischen Fortschritts die leistungsorientierte Entlohnung in der Landwirtschaft mit großen Herausforderungen für die Betriebsleitung behaftet ist. Auch für nachfolgende Arbeiten besteht noch Forschungsbedarf. Die vorliegende Arbeit verfolgt verschiedene theoretische Ansätze zur leistungsorientierten Entlohnung, die durch den explorativen Charakter der Untersuchung begründet sind. Folgende Arbeiten sollten sich auf einen theoretischen Ansatz konzentrieren, um diesen detaillierter untersuchen zu können. Fußend auf den Ergebnissen der empirischen Analyse könnten zum Beispiel Experimente $\mathrm{zu}$ den individual- und sozialpsychologischen Theorien mit 
landwirtschaftlichen Arbeitnehmern durchgeführt werden, die die Konstrukte „intrinsische Motivation“, „Fairness“, „Reziprozität“ und „Verlustaversion“ näher untersuchen. 


\section{Zusammenfassung}

Durch die zunehmende Bedeutung von Lohnarbeitskräften in der deutschen Landwirtschaft gewinnen personalwirtschaftliche Fragestellungen für Betriebsleiter und andere Interessenten, wie z.B. landwirtschaftliche Berater, an Relevanz. Ein zentrales Forschungsgebiet des Personalmanagements ist die Motivation von Mitarbeitern. Anreizsysteme sollen durch ihre immaterielle oder materielle Ausgestaltung zur Erhöhung der Mitarbeiter beitragen. Die vorliegende Arbeit befasst sich mit den Gestaltungsmöglichkeiten materieller Anreizsysteme und der Frage, unter welchen Bedingungen leistungsorientierte Entlohnungssysteme in der Landwirtschaft Anwendung finden können und welche Erfahrungen die betroffenen Personen mit dieser Vergütungsform gesammelt haben. Sie basiert auf einer schriftlichen Betriebsleiter- und Mitarbeiterbefragung.

Das zweite Kapitel ist den Rahmenbedingungen der leistungsorientierten Entlohnung in der Landwirtschaft gewidmet. Zunächst werden die Besonderheiten der Landwirtschaft beschrieben, die sie von anderen Wirtschaftszweigen unterscheidet. Die Abhängigkeit von der Natur, die Bodengebundenheit der Produktion und die daraus resultierenden Flexibilitätserfordernisse im Arbeitsprozess beeinflussen die Möglichkeiten einer leistungsorientierten Lohngestaltung in der Landwirtschaft.

Der nächste Abschnitt dieses Kapitels ist der Beschäftigungssituation in der Landwirtschaft gewidmet. Ein niedriges Lohnniveau und vergleichsweise lange und unregelmäßige Arbeitszeiten sind wichtige Determinanten für die Beschäftigung in der Landwirtschaft. Es wird außerdem deutlich, dass sich die Relevanz der familienfremden Arbeitskräfte zwischen den alten und neuen Bundesländern unterscheidet. Im früheren Bundesgebiet herrscht nach wie vor die Arbeitsverfassung des Familienbetriebs vor. Der Anteil der Lohnarbeitskräfte an den Beschäftigten in der Landwirtschaft nimmt jedoch durch den fortschreitenden Strukturwandel zu. In den neuen Bundesländern dominiert hingegen die Lohnarbeitsverfassung. Die meisten familienfremden Arbeitskräfte sind, trotz der starken Umstrukturierungsmaßnahmen nach der Wende, in den Betrieben der Rechtform juristischer Personen beschäftigt. Ein möglicher Fachkräftemangel, der von einigen Experten prognostiziert wird, ist nach Einschätzung der befragten Betriebsleiter eher in den alten als in den neuen Bundesländern zu 
erwarten. Hier sind es vor allem die Veredlungsbetriebe, in denen zukünftig ein Engpass an qualifizierten Facharbeitskräften prognostiziert wird.

Personalwirtschaftliche Maßnahmen werden beeinflusst von den arbeitsrechtlichen Rahmenbedingungen unter denen sie Anwendung finden. Diese werden im letzten Teil des zweiten Kapitels kurz skizziert.

Die optimale Gestaltung von Anreizsystemen und die Einsatzmöglichkeiten leistungsorientierter Entlohnungssysteme werden in der ökonomischen Literatur zum Teil sehr kontrovers diskutiert. Die Aussagen verschiedener theoretischer Strömungen sind in diesem Punkt sehr widersprüchlich und führen $\mathrm{zu}$ keinem einheitlichen Ergebnis. Drei ökonomische Richtungen bilden den theoretischen Hintergrund der empirischen Analyse. Die Grundzüge dieser theoretischen Ansätze und ihre Implikationen für die Gestaltung von Anreizsystemen werden im dritten Kapitel vermittelt.

Neoklassischen Forschungsansätzen liegt das Menschenbild des rational handelnden eigennützigen homo oeconomicus zugrunde. Modifikationen lassen begrenzte Rationalität und Informationsasymmetrien zu und führen zum Menschenbild der Neuen Institutionenökonomie. Unter den institutionenökonomischen Ansätzen ist die Prinzipal-Agenten-Theorie (PA) wohl einer der prominentesten ökonomischen Ansätze zur Lösung von Anreizproblemen. Sie stellt der Betriebswirtschaft ein logisches Modell zur optimalen Lohngestaltung zur Verfügung und überzeugt durch ihre mathematisch präzise Lösung. Sie befürwortet den Einsatz materieller Anreize. Die optimale Vergütungsform hängt vor allem von der Risikoaversion der beteiligten Akteure ab. Aus der Transaktionskostentheorie lässt sich für eine leistungsorientierte Lohngestaltung folgern, dass die Messbarkeit des Arbeitseinsatzes eine wichtige Erfolgsbedingung ist.

Personalökonomische Modelle wie die Effizienzlohntheorie von SHAPIRO UND STIGLITZ bauen auf die gleichen Grundannahmen wie die des PA-Ansatzes (z.B. Annahmen zur Risikoaversion der Vertragsparteien, Nutzenniveau) auf. Effizienzlohntheoretiker betrachten ein überdurchschnittliches Lohnniveau in Unternehmen als Motivationsinstrument, das durch verschiedene Erklärungsmodelle begründet werden kann. 
Die Aussagen der individualpsychologischen Motivationstheorien zum Stellenwert verschiedener Anreizsysteme hängen vom jeweiligen theoretischen Konzept ab. Zu den Inhaltstheorien zählt Herzbergs Zweifaktoren-Theorie. HERZBERG unterscheidet so genannte Hygienefaktoren von Motivatoren. Hygienefaktoren, hierzu zählt u.a. die Bezahlung, können einzig Unzufriedenheit verhindern, sie leisten jedoch keinen Beitrag zur Motivationsförderung. Diese kann nur durch die Motivatoren erreicht werden, dieses sind Faktoren, die sich auf den Arbeitsinhalt beziehen. Dass faire Behandlung im Vergleich zu einer Referenzgruppe einen großen Einfluss auf den Erfolg von Entlohnungssystemen hat, findet sich in zahlreichen Beiträgen zum Thema Personalmotivation wieder und hat auch in neoklassisch geprägte Monographien Eingang gefunden. Die Bedeutung distributiver Gerechtigkeit für die Personalmotivation basiert auf Adams' Equity Theorie, die den Inhaltstheorien der Personalmotivation zuzuordnen ist.

Nach dem Modell von PORTER UND LAWLER sind für den Erfolg eines Anreizsystems die Wertschätzung des Anreizes durch den Mitarbeiter und die subjektive Wahrscheinlichkeit durch Anstrengung eine versprochene Belohnung auch zu erreichen von zentraler Bedeutung. Persönliche Fähigkeiten und die Rollenwahrnehmung der Mitarbeiter spielen eine Rolle in der Frage, inwieweit Anstrengung auch zur erwünschten Belohnung führt.

Spätestens seit der Verleihung des Nobelpreises für Wirtschaftswissenschaften an den Psychologen Daniel Kahneman ist die Bedeutung sozialpsychologischer Erkenntnisse für die Gestaltung von Anreizsystemen in der ökonomischen Forschung weitgehend anerkannt. Die Erklärung für viele Verhaltensanomalien - aus neoklassischer Sicht liegt in der Orientierung der Menschen an einem neutralen Referenzpunkt. Verluste im Vergleich zum Referenzpunkt werden dabei stärker bewertet als mögliche Gewinne (Verlustaversion). Die Verlustaversion ist eine wichtige Implikation der Prospect Theory, einer Entscheidungstheorie für Entscheidungen unter Unsicherheit, mit der die Psychologen Daniel Kahneman und Amos Tversky eine Alternative zur von NeumannMorgenstern'schen bieten. Die Orientierung am Referenzpunkt führt zu empirisch robusten Verhaltensabweichungen vom Rationalprinzip wie dem Endowment Effect und dem Status-Quo-Bias. 
Experimentelle Ökonomen konnten außerdem zeigen, dass intrinsische Motivation durch extrinsische Motivation verdrängt werden kann und soziale Normen und Reziprozität für das Verhalten von Menschen nicht zu unterschätzen sind.

Im letzten Teil des Kapitels wird ein Modell zu den Erfolgsbedingungen leistungsorientierter Entlohnung vorgestellt, das auf den vorgestellten theoretischen Ansätzen basiert.

Das Kapitel 4 zeigt verschiedene Gestaltungsmöglichkeiten materieller Anreizsysteme auf. Die Charakteristika und Einsatzbedingungen input- und outputbasierter Entlohnungssysteme werden dargestellt, um die Ergebnisse der empirischen Analyse in Bezug auf die Verwendung der Entlohnungssysteme einordnen zu können.

Konzeption, Durchführung und Ergebnisse der empirischen Analyse werden im fünften Kapitel dargestellt. Die empirische Analyse beruht auf einer Befragung von 260 Betriebsleitern landwirtschaftlicher Lohnarbeitsbetriebe im gesamten Bundesgebiet und von 124 Mitarbeitern in Niedersachsen. Sie wurden als schriftliche Befragungen konzipiert, um eine breite Erhebung des Status Quo zu ermöglichen. In Hinblick auf die im dritten Kapitel vorgestellten Erfolgsbedingungen leistungsorientierter Entlohnung zeigt sich, dass die befragten Mitarbeiter über eine hohe intrinsische Motivation verfügen. Finanzielle Anreize werden von den Mitarbeitern geschätzt, sie sind aber nicht das wichtigste Motivationsinstrument. Wichtiger sind ein sicherer Arbeitsplatz und ein gutes Betriebsklima. Die Faktorenanalyse und Korrelationsanalysen zeigen, dass die Freude an der Arbeit bei den befragten Mitarbeitern besonders durch ein Gefühl der Einbindung in betriebliche Geschehnisse, Feedback und eine Würdigung der Arbeitsleistung hervorgerufen wird. Dieses Ergebnis stützt Herzbergs Zweifaktorentheorie. Die hohe Motivation der Mitarbeiter wird auch in der Betriebsleiterbefragung bestätigt.

Die Analyse der verwendeten Entlohnungssysteme verdeutlicht, dass leistungsorientierte Entlohnungssysteme in der Landwirtschaft insgesamt eine geringe Rolle spielen. Sie werden häufiger in der Tierproduktion als in der Pflanzenproduktion angewendet, da es im Ackerbau besonders schwierig ist, einfache und objektiv messbare Leistungsindikatoren zu finden. Die empirische Analyse zeigt außerdem, dass die Wahrscheinlichkeit, dass ein Betriebsleiter bereits Erfahrung mit leistungsorientierter Entlohnung gemacht hat, durch verschiedene Einflussfaktoren 
bestimmt wird. Hierzu zählen die Betriebsgröße und die Betriebsleitereinstellung zur Vorzüglichkeit verschiedener Anreizsysteme. Mit steigender Betriebsgröße, gemessen in der Größe der Tierbestände und Anzahl der Fremdarbeitskräfte wird es schwieriger, den individuellen Arbeitseinsatz der Mitarbeiter zu bestimmen, so dass das PrinzipalAgenten-Problem zum Tragen kommt. Leistungsorientierte Entlohnung kommt häufiger zum Einsatz. Die empirischen Ergebnisse sind in diesem Punkt konsistent mit der ökonomischen Theorie.

Mit Hilfe der Clusteranalyse wurden drei Gruppen von Betriebsleitern identifiziert, die bereits Erfahrung mit leistungsorientierter Entlohnung gesammelt haben und sich in verschiedenen Punkten unterscheiden. Das erste Cluster zeichnet sich durch eine sehr positive Einstellung zur leistungsorientierten Entlohnung aus. Die möglichen Nachteile dieser Entlohnungsform, wie die Wahrnehmung von Fremdkontrolle oder eine negative Auswirkung auf die Stimmung im Team werden von den Betriebsleitern dieses Clusters nicht bestätigt. Diese Betriebsleiter bewirtschaften überwiegend Einzelunternehmen oder Personengesellschaften konventioneller Wirtschaftsweise. Das zweite Cluster hat eine ähnliche Betriebstruktur in Bezug auf die Größe und Rechtsform wie das erste Cluster. Die Betriebsleiter beurteilen Leistungslohnsysteme aber wesentlich kritischer als die erste Gruppe. Interessanterweise finden sich in diesem Cluster eine hohe Anzahl ökologisch wirtschaftender Betriebe. Das dritte Cluster besteht vor allem aus den Betriebsleitern der juristischen Personen und unterscheidet sich von den beiden anderen Clustern durch die geringste Zustimmung zur Motivationswirkung leistungsabhängiger Vergütung. Diese Betriebsleiter differenzieren die Einkommen ihrer Mitarbeiter außerdem stärker als die anderen Betriebsleiter.

Die Erfahrung der Mitarbeiter mit Leistungslöhnen ist eher positiv. Dieses Ergebnis ist jedoch mit Vorsicht zu interpretieren, da bei den Ergebnisworkshops deutlich wurde, das viele Mitarbeiter den Unterschied zwischen leistungs- und erfolgsorientierter Entlohnung nicht kennen und alle Indikatoren, die die Betriebsleiter zur Leistungsbemessung einsetzen als mehr oder weniger willkürlich und unfair einschätzen. 
Handlungsempfehlungen und Perspektiven für die leistungsabhängige Vergütung in der Landwirtschaft werden im sechsten Kapitel abgeleitet. Zunächst wird ein Leitfaden vorgestellt, an dem sich Betriebsleiter, die ermitteln wollen, ob leistungsorientierte Entlohnung für sie in Frage kommt, orientieren können. Im Anschluss werden mögliche Leistungsindikatoren im Ackerbau, in der Milch- und Schweineproduktion sowie bei der Biogas- und Stromerzeugung vorgestellt und diskutiert. Im folgenden Abschnitt wird für die oben genannten Betriebszweige eine Sensitivitätsanalyse betriebswirtschaftlicher Erfolgskennzahlen durchgeführt. Es wird jeweils die Auswirkung einer fünfprozentigen Leistungssteigerung, die durch einen Leistungslohn von $10 \%$ zusätzlich zum Grundlohn induziert werden soll, auf die Wirtschaftlichkeit des Betriebszweigs diskutiert. Es wird deutlich, dass unter den definierten, stark vereinfachenden Annahmen, das angenommene Leistungslohnsystem immer wirtschaftlich ist, obwohl die Lohnstückkosten steigen. Im letzten Teil des Kapitels werden die Perspektiven für eine leistungsabhängige Entlohnung in der Landwirtschaft abgeleitet. Grundsätzlich ist davon auszugehen, dass die Messbarkeit des individuellen Leistungsbeitrags der Mitarbeiter durch die „Precision Agriculture“ zunehmen wird. Stochastische Einflüsse, wie z. B. die Witterung werden aber weiterhin dazu führen, dass Leistungslöhne nur unter sehr einschränkenden Voraussetzungen in der Landwirtschaft angewendet werden können. 


\section{Literaturverzeichnis}

Adams, S. (1965): Inequity in social exchange. Advances in experimental social psychology; New York.

Anders, H. (2002): Strukturwandel in der Landwirtschaft Thüringens - Teil 2: Beschäftigte in den landwirtschaftlichen Betrieben. In: Statistisches Monatsheft, Thüringer Landesanstalt für Statistik, 9. Jahrgang, Heft 7, S. 3846.

Agrocom (2006): Eine Handheld-Anwendung - vielfache Funktionalitäten. Download vom 18.12.2006 unter http://www.agrocom.de/index.php?a=524

Ait Abdelmalek, A. (2000): L'exploitation familiale agricole: entre permanence et évolution. In: Economie Rurale, Band 255-256, Januar/April 2000, S. 40-52.

Akerlof, G. A. und Yellen, J. L. (1990): The fair wage-effort hypothesis and unemployment. In: The Quarterly Journal of Economics, Band 105, Heft 2, S. 255-283.

Allen, D. W. und Lueck, D. (2003): The nature of the farm: contracts, risk, and organization in agriculture; Cambridge.

Andermann, G. und Schmitt, G. (1996): Die Bestimmungsgründe der Beschäftigung in der Landwirtschaft - Eine quantitative Analyse des Arbeitskräftebestandes der Landwirtschaft im früheren Bundesgebiet 1971 bis 1991. Mitteilungen aus der Arbeitsmarkt- und Berufsforschung, 29. Jahrgang, Heft 4, S. 630655.

Backes-Gellner, U. et al. (2001): Personalökonomik - Fortgeschrittene Anwendungen für das Management; Stuttgart.

Backhaus et al. (2000): Multivariate Analyseverfahren, 9. Auflage; Berlin.

Balmann, A. et al. (1996): Beschäftigungswirkungen der Umstrukturierung der ostdeutschen Landwirtschaft, Working Paper 23, Humboldt-Universität zu Berlin.

Becker, F. G. (1995): Anreizsysteme als Führungsinstrumente. In: A. Kieser et al.: Handwörterbuch der Führung; Stuttgart, Sp. 34-45.

Beckmann, V. (2000): Transaktionskosten und institutionelle Wahl in der Landwirtschaft: zwischen Markt, Hierarchie und Kooperation; Berlin.

Billikopf, G. (2003): Labor Management in Agriculture: Cultivating Personnel Productivity? Modesto. 
Bitsch, V. (2003): Management of Human Resources in Agriculture. In: Balmann, A. und Lissitsa, A. (Hrsg.): Large Farm Management - Studies on the Agricultural and Food Sectors in Central and Eastern Europe, Bergen/Dumme, S. 91-111.

Blanc, M. und Perrier-Cornet, P. (1999): Emploi agricole: les cadres d'analyse à l'épreuve des dynamiques actuelles. In: Economie Rurale September/Oktober 1999, S. 8-14.

BMVEL (verschiedene Jahrgänge): Statistisches Jahrbuch über Ernährung, Landwirtschaft und Forsten, Münster-Hiltrup.

Bofinger, P. und Schmidt, R. (2003): Nobelpreis für Wirtschaftswissenschaften 2002 an Daniel Kahneman und Vernon L. Smith. In: Wirtschaftswissenschaftliches Studium, Band 32, Heft 2, S. 107-111.

Bokelmann, W. (2000): Personalmanagement. In: Odening, M. und Bokelmann, W. (Hrsg.): Agrarmanagement; Stuttgart.

Bolton, G. und Ockenfels, A. (2000): ERC - A Theory of Equity, Reciprocity and Competition. In: American Economic Review, Band 90, Heft 1, S. 166-193.

Brandes, W. et al. (1997): Produktions- und Umweltökonomik Band I; Stuttgart.

Brandes, W. (1995): Antwort auf Günther Schmitt - eine Polemik. In: Agrarwirtschaft, 44. Jahrgang, Heft 2, S. 120-123.

Brandes, W. und Odening, M. (1992): Investition, Finanzierung und Wachstum in der Landwirtschaft; Stuttgart.

Budde, J. und Wielenberg, S. (1997): Rank-Oder-Tournaments als Entlohnungsschemata. In: Zfbf, 49. Jahrgang, 11/1997, S. 923-950.

Bühner, M. (2004): Einführung in die Test- und Fragebogenkonstruktion; München.

Campbell, C. M. und Kamlani, K. (1997): The reasons for wage rigidity: evidence from a survey of firms. In: The Quarterly Journal of Economics, Band 112, Heft 3, S. 759-789.

Dahms, V. (1998): Beschäftigungsentwicklung in der Land- und Forstwirtschaft Mecklenburg-Vorpommerns - Ergebnisse des IAB-Betriebspanels 1997, SÖSTRA Sozialökonomische Strukturanalysen e.V.; Berlin.

Davier, Z. von et al. (2006): Organisatorische und technologische Innovationen in der Landwirtschaft. Schriftenreihe der landwirtschaftlichen Rentenbank, Band 21; Frankfurt am Main.

Debertin, D. L. (1986): Agricultural Production Economics; New York. 
Deci, E. L. et al. (1999): A meta-analytic review of experiments examing the effect of extrinsic rewards on intrinsic motivation. In: Psychological Bulletin, Band 125, Heft 6, S. 627-668.

Diaz-Bone, R. und H. Künemund (2003): Einführung in die binäre logistische Regression, Mitteilungen aus dem Schwerpunkt Methodenlehre, Heft Nr. 56, Institut für Soziologie der Freien Universität Berlin.

Diekmann (2004). Empirische Sozialforschung - Grundlagen, Methoden, Anwendungen; Reinbek bei Hamburg.

Dippmann, L. (2004): So organisieren Sie Arbeitszeit. In: DLG-Mitteilungen, 12/2004, S. 32-35.

DLG (2007): Dieseleinsparung in der Pflanzenproduktion, Merkblatt 339; Frankfurt am Main.

DLG (2006): Betriebszweigabrechnung für Biogasanlagen: Einheitliche Abrechnung und Erfolgskennzahlen für Biogasanlagen aller Rechtsformen; Frankfurt am Main.

Doluschitz, R. (2001): Personalmanagement und Landwirtschaft; Bonn.

Doluschitz, R. et al. (1996): Neue Managementformen und -inhalte für Unternehmen des Produktionsbereichs im betriebsstrukturellen Anpassungsprozeß der Landwirtschaft in Mecklenburg-Vorpommern. Institut für Landwirtschaftliche Betriebslehre der Universität Hohenheim; Stuttgart.

Eisenführ, F. und Theuvsen, L. (2004): Einführung in die Betriebswirtschaftslehre; 4. Auflage, Stuttgart.

Elschen, R. (1991): Gegenstand und Anwendungsmöglichkeiten der Agency-Theorie. In: ZfBf, 43. Jahrgang, 11/1991, S. 1002-1012.

Evers, H. (1995): Entgeltpolitik für Führungskräfte. In: Kieser, A. (Hrsg.): Handwörterbuch der Führung, 2. Auflage, Stuttgart, Sp. 297-306.

Fasterding, F. und Rixen, D. (2005): Analyse der Beschäftigungsmöglichkeiten im Agrarsektor Deutschlands und Beschäftigungseffekte agrarpolitischer Maßnahmen. Arbeitsberichte des Bereichs Agrarökonomie der Bundesforschungsanstalt für Landwirtschaft, 5/2005; Braunschweig.

Fasterding, F. (2003): Fachkräftemangel in der Landwirtschaft? In: B\&B agrar, 12/2003, S. 350-353.

F.A.Z. (2007): Wenn Geburtstage sich nicht lohnen, Ausgabe vom 24.03.2007, S. C1. 
F.A.Z. (2005): Variable Vergütung bei Arbeitnehmern unbeliebt, Ausgabe vom 10.09.2005, S. 59.

F.A.Z. (2003): Ohne Preis kein Fleiß - Personalökonomie: Leistung muss sich lohnen. Ausgabe vom 1.08.2003, S. 17.

Fehr, E. und Gächter, S. (2000): Fairness and retaliation: the economics of reciprocity. In: Journal of Economic Perspectives, Band 14, Heft 3, S. 159-181.

Fehr, E. und Goette, L. (2005): Do workers work more if wages are high? Evidence from a randomized field experiment. Working Paper Nr. 125 des Instituts für Empirische Wirtschaftsforschung der Universität Zürich, September 2005.

Fehr, E. und Schmidt, K. (2001): Theories of Fairness and Reciprocity - Evidence and Economic Applications, Download vom 13.02.04 unter http://www.iew.unizh.ch/wp/iewwp075.pdf

Festinger, L. (1957): A theory of cognitive dissonance; Evanston.

Fock, T. und Fechner, J. (2003): Der Fachkräfte- und Bildungsbedarf in Brandenburg bis 2015. In: B\&B agrar, 6/2003, S. 184-186.

Fock, T. et al. (1996): Ermittlung des zukünftigen Arbeitskräftebedarfs in der Agrarwirtschaft Mecklenburg-Vorpommerns und Konsequenzen für die Berufsbildung, Bericht der Fachhochschule Neubrandenburg, 1996.

Franke, G. (1993): Agency-Theorie. In: W. Wittmann: Enzyklopädie der Betriebswirtschaftslehre, Handwörterbuch der Betriebswirtschaft, Band 1, Sp. 37-49. Stuttgart.

Frey, B. S. und Jegen, R. (2000): Motivation crowding theory: a survey of empirical evidence, revised version, Working Paper No. 49, Download vom 19.08.2006 unter http://e-collection.ethbib.ethz.ch/ecolpool/incoll/incoll_562.pdf.

Frey, B. S. und Oberholzer-Gee, F. (1997): The cost of price incentives: an empirical analysis of motivation crowding-out. In: American Economic Review, Band 87, Heft 4, S. 746-755.

Frey, B. S. und Osterloh, M. (1997): Sanktionen oder Seelenmassage? Motivationale Grundlagen der Unternehmensführung. In: DBW, Band 57, Heft 3, S. 307321.

Frey, B. S. et al. (2001): Grenzen variabler Leistungslöhne - Die Rolle intrinsischer Motivation. In: Jost, P.-J. (Hrsg.): Die Prinzipal-Agenten-Theorie in der Betriebswirtschaftslehre; Stuttgart, S. 561-577. 
Friedrichs, J. (1985): Methoden empirischer Sozialforschung, 13. Auflage; Opladen.

Fromm, S. (2005): Binäre logistische Regression - Eine Einführung für Sozialwissenschaftler mit SPSS für Windows. In: Schulze, G. und Akremi, L. (Hrsg.): Bamberger Beiträge zur empirischen Sozialforschung, Nr. $11 / 2005$.

Gabler, D. (1995): Entwicklungsabschnitte der Landwirtschaft in der ehemaligen DDR; Berlin.

Gächter, S. (2003): Unvollständige Verträge und Effizienzlöhne im Experiment. In: Held, M. (Hrsg.): Experimente in der Ökonomik, Marburg, S. 39-67.

Gottensträter, A. (2003): Gute Vererber lohnen sich. In: DLG-Mitteilungen, 10/2003, S. $12-15$.

Griesel, Heiko (2004): Mitarbeiter fair entlohnen. In: Land und Forst, 11/2004 vom 11.3.2004, S. 6-7.

Harms, J. H. (2004): Untersuchungen zum Einsatz verschiedener Varianten des Tierumtriebs in automatischen Melksystemen (Einboxenanlagen); München.

Heller, G. (2003): Mit Klischees aufräumen! Die Vergütung in Agrarunternehmen sollte überlegt gestaltet werden. In: Neue Landwirtschaft, 4/2003, S. 16-18.

Herzberg, F. (1987): One more time: How do you motivate employees? In: Harvard Business Review, Band 65, Heft 5, S. 109-120.

Hess, S. (2002): The employment of seasonal farm workers from Central and Eastern European countries in German agriculture, Masterarbeit, Universität Göttingen.

Jensen, M. C. und Meckling, W. H. (1976): Theory of the Firm: Managerial behavior, agency costs and ownership structure. In: Journal of Financial Economics, Band 3, S. 305-360.

Jost, P.-J. (2001): Die Prinzipal-Agenten-Theorie in der Betriebswirtschaftslehre; Stuttgart.

Kahneman, D. und Knetsch, J. L. (1992): The Endowment Effect, Loss Aversion, and Status Quo Bias. In: R. H. Thaler (Hrsg.): The Winner's Curse - Paradoxes and Anomalies of Economic Life, S. 63-78; New York.

Kahneman, D. et al. (1991): Anomalies: The Endowment Effect, Loss Aversion and Status Quo Bias. In: Journal of Economic Perspectives, Band 5, Heft 1, S. 193-206. 
Knetsch, J. L. und Sinden, J. A. (1984): Willingness to Pay and Compensation Demanded: Experimental Evidence of an Unexpected Disparity in Measures of Value. In: The Quarterly Journal of Economics, Band 99, Heft 3, S. 507521.

Knoeber, C. R. und Thurman, W. N. (1995): "Don't Count Your Chickens...": Risk and Risk Shifting in the Broiler Industry." In: American Journal of Agricultural Economics, Band 77, Heft 3, S. 486-496.

Knoeber, C.R. und Thurman, W. N. (1994): Testing the theory of tournaments: an empirical analysis of broiler production. In: Journal of Labor Economics, Band 12, Heft 2, S. 155-179.

Koesling, T. (2006): Mitarbeiter werden oft entweder über- oder unterfordert. In: DLGMitteilungen, 7/2006, S. 26.

Koschwitz, D. und Dippmann, L. (1997): Mit wenig Aufwand viel erreichen? Leistungsorientierte Lohnsysteme in Agrarunternehmen. In: Neue Landwirtschaft, 4/97, S. 18-21.

Kowalczyk, C. und Schmidt, D. (2006): Top-Betrieb, ,halber Ertrag“. In: DLGMitteilungen, 10/2006.

Kromka, F. (2004): Das Flow-Erlebnis in der Landarbeit: warum Landwirte lieber und länger arbeiten als Nichtlandwirte. Zeitschrift für Agrargeschichte und Agrarsoziologie, Band 52, Heft 2, S. 78-102.

Kübler, H. (2007): Bits und Bytes zwischen Acker und PC, Folienvortrag vom 10.01.2007, DLG-Wintertagung 2007 in München. Download vom 06.05.2007 unter http://download.dlg.org/pdf/wita2007/PR_Kuebler.pdf.

Kuhlmann, F. (2003): Betriebslehre der Agrar- und Ernährungswirtschaft, 2. Auflage; Frankfurt am Main.

Krüger, H. (2005): Der landwirtschaftliche Arbeitsmarkt im Bundesgebiet West. Ergebnisse aus der Analyse der Zahlen der Bundesagentur für Arbeit. Präsentiert auf der Sitzung des Fachausschuss "Arbeitnehmer im Agrarbereich" des Verbands der Landwirtschaftskammern e.V. am 28.04.2005 in Wehnen.

LANDWIRTSCHAFTSKAMMER SCHLESWIG-HolsteIn (2007): Schweinereport 2007. Download vom 28.04.2007 unter http://www.lwksh.de/fileadmin/user_upload/Downloads/Schriftenreihe/Schweinereport_200 6.pdf. 
LANDWIRTSCHAFTSKAMmer SChLeSWIG-HOlsteIN (2005a): Tierreport 2004,

Mitteilungen der Landwirtschaftskammer Schleswig-Holstein, Heft 580.

LANDWIRTSCHAFTSKAMMER SCHLESWIG-Holstein (2005b): Marktfruchtreport 2005, Mitteilungen der Landwirtschaftskammer Schleswig-Holstein, Heft 582.

Langbehn, C. (1994): Produktionsstrukturen und Unternehmensformen in der Landwirtschaft: Auch fünf Jahre nach der Wende hält die Diskussion an. Agrarwirtschaft, 43. Jahrgang, Heft 11, S. 381-382.

Laux, H. (1988): Grundprobleme der Ermittlung optimaler erfolgsabhängiger Anreizsysteme. In: ZfB, Band 58, Heft 1, S. 24-36.

Laux, H. und Liermann, F. (2003): Grundlagen der Organisation: die Steuerung von Entscheidungen als Grundproblem der Betriebswirtschaftslehre; Berlin/ Heidelberg.

Lazear, E. P. (2000a): The Future of Personnel Economics. In: The Economic Journal, Band 110, November 2000, S. F611-639.

Lazear, E. P. (2000b): Performance Pay and Productivity. In: American Economic Review, Band 90, Nr. 50, 1346-1361.

Mann, K.-H. (2006): Zu teures Lieblingskind. In: DLG-Mitteilungen, 5/2006, S. 28-30.

Mann, K.-H., et al. (2006): Ohne Mitarbeiter über den Winter. In: DLG-Mitteilungen, 8/2006, S. 30-33.

Martens, K.P. (1993): Arbeitsrecht und Unternehmung. In: Wittmann et al. (Hrsg.): Handwörterbuch der Betriebswirtschaft, Sp. 151-170.

Maslow, A. H. (1996): Motivation und Persönlichkeit; Hamburg.

Milgrom, P. und Roberts, J. (1992): Economics, Organization and Management; New Jersey.

Ministerium für Landwirtschaft, Umwelt und ländliche Räume des Landes SchleswigHolstein (2007): Agrarbericht des Landes Schleswig-Holstein Fördermittel, Preisausgleichszahlungen der EU für die Landwirtschaft 2002 und 2003, Download vom 29.04.2007 unter http://www.umwelt.schleswigholstein.de/servlet/is/38121/ausgleich.htm.

Moretti, E. und Perloff, J. M. (2002): Efficiency Wages, Deferred Payments, and Direct Incentives in Agriculture. In: American Journal of Agricultural Economics, Band 84, Heft 4, S. 1144-1155.

Müller, C. (1995): Agency-Theorie und Informationsgehalt: der Beitrag des normativen Prinzipal-Agenten-Ansatzes zum Erkenntnisfortschritt der 
Betriebswirtschaftslehre. In: Die Betriebswirtschaft, Band 55, Heft 1, S. 6176.

Nause, G. (2003): Zur Entwicklung der in den landwirtschaftlichen Betrieben Deutschlands beschäftigten Arbeitskräfte 1991 bis 2001. In: Statistisches Bundesamt Wirtschaft und Statistik, Heft 4/2003, S. 301-313.

Neumann, H. (2006): Biogas: Fütterungsberatung bringt mehr Gas und Geld. In: top agrar, 4/2006, S. 130-132.

Odening, M. (1994): Zur Bedeutung von Prinzipal-Agenten-Modellen in der agrarökonomischen Forschung. In: Agrarwirtschaft, 43. Jahrgang, Heft 7, S. 271-278.

Otsuka, K. et al. (1992): Land and labor contracts in agrarian economies: theories and facts. In: Journal of Economic Literature, Band 30, Heft 4, S. 1965-2018.

Picot, A. (1991): Ökonomische Theorien der Organisation - Ein Überblick über neuere Ansätze und deren betriebswirtschaftliches Anwendungspotential. In: Ordelheide, D. (Hrsg.): Betriebswirtschaftslehre und ökonomische Theorie, S. 143-160; Stuttgart.

Pollak, R. A. (1985): A Transaction Cost Approach to Families and Households. In: Journal of Economic Literature, Band 23, Heft 2, S. 581-608.

Porter, L. W. und Lawler, E. E. (1968): Managerial attitudes and performance; Homewood.

Rahmann, G. et al. (2004): Bundesweite repräsentative Erhebung und Analyse der verbreiteten Produktionsverfahren, der realisierten Vermarktungswege und der wirtschaftlichen sowie sozialen Lage ökologisch wirtschaftender Betriebe und Aufbau eines bundesweiten Praxis-Forschungs-Netzes, Bericht, Institut für ökologischen Landbau und Institut für Betriebswirtschaft, Bundesforschungsanstalt für Landwirtschaft (FAL).

Rathmann, C. et al. (2005): Arbeitsmarktpartizipation landwirtschaftlicher Haushalte: eine empirische Analyse für Schleswig-Holstein. Beitrag der GEWISOLATagung 2005 in Göttingen vom 6.10.2005. Download vom 07.05.2007 unter http://www.uni-goettingen.de/de/sh/28441.html.

Reisch, E. (1993): Landwirtschaft. In: Wittmann, W. (Hrsg.): Enzyklopädie der Betriebswirtschaftslehre, Band 1, Sp. 2529-2545; Stuttgart.

Reisch, K. (1992): Zeit- und Pauschallohn. In: Gaugler, E. und Weber, W. (Hrsg.): Handwörterbuch des Personalwesens, 2. Auflage, Sp. 2359-2369; Stuttgart. 
Remminghorst, K. (2000): Unternehmensformen in der Landwirtschaft - eine vergleichende empirische Analyse; Kiel.

Richardi, R. (2006): Einführung. In: DTV (Hrsg.): Arbeitsgesetze: mit den wichtigsten Bestimmungen zum Arbeitsverhältnis, Kündigungsrecht, Arbeitsschutzrecht, Berufsbildungsrecht, Tarifrecht, Betriebsverfassungsrecht, Mitbestimmungsrecht und Verfahrensrecht, Textausgabe; München.

Rinderreport Rheinland-Pfalz (2007): BZA Rind 2006. Betriebszweigauswertung der Milchviehberatungsringe in Rheinland-Pfalz, Arbeitsgemeinschaft der Milchviehberatungsringe Rheinland-Pfalz (Hrsg.); Mainz.

Roumasset, J. (1995): The nature of the agricultural firm. In: Journal of Economic Behavior \& Organization, Band 26, Heft 2, S. 161-177.

Sächsische Landesanstalt für Landwirtschaft (2005): Schweine-Report 2004. Wirtschaftlichkeitsbericht zur sächsischen Schweineproduktion; Leipzig.

Sächsische Landesanstalt für Landwirtschaft (2002): Personalmanagement in landwirtschaftlichen Unternehmen - Managementunterlage; Leipzig.

Samuelson, W. und Zeckhauser, R. (1988): Status quo bias in decision making. In: Journal of risk and uncertainty, Band 1, Heft 1, S. 7-59.

Schaper, C. (2006): Wachstumsoptionen landwirtschaftlicher Familienbetriebe - eine empirische Analyse im Kontext des technologischen Fortschritts und Arbeitskrafteinsatzes in der Landwirtschaft, Masterarbeit, Universität Göttingen.

Schattauer, A. und Weiland, P. (2005): Grundlagen der anaeroben Fermentation. In: Fachagentur Nachwachsende Rohstoffe e.V. (Hrsg.): Handreichung Biogasgewinnung und -erzeugung, Gülzow.

Schmitt, G. (1995): Anmerkungen und Erwiderung zum Beitrag „Entwicklung und Entwicklungstendenzen auf der Ebene des Einzelbetriebes“ von Wilhelm Brandes. In: Agrarwirtschaft, 44. Jahrgang, Heft 2, S. 117-120.

Scholz, Christian (2000): Personalmanagement: informationsorientierte und verhaltenstheoretische Grundlagen; 5. Auflage, München.

Shapiro, C. und Stiglitz, J. E. (1984): Equilibrium Unemployment as a Worker Discipline Device. In: American Economic Review, Band 74, Heft 3, S. 433 444. 
Sheldon, G. (2007): Unterlagen zur Lehrveranstaltung Arbeitsmarktökonomie im Wintersemester 2006/2007, Forschungsstelle Arbeitsmarkt- und Industrieökonomik, Universität Basel, Download vom 12.12.2006 unter: http://www.wwz.unibas.ch/fai/pages/arbeitsmarktoekonomik/entlohnung.pdf

Skott, P. (2005): Fairness as a source of hysteresis in employment and relative wages. In: Journal of Economic Behavior \& Organization, Band 57, Heft 3, S. 305331.

Statistisches Bundesamt (verschiedene Jahrgänge): Fachserie 3, Reihe 2.2 (bis 1997) und Reihe 2.18 (ab 1997); Wiesbaden.

Statistisches Bundesamt (verschiedene Jahrgänge): Fachserie 16, Reihe 2.1 „Löhne und Gehälter, Produzierendes Gewerbe“.

Stabler, J.C. und Olfert, M. R. (2000): Public policy in the 21st century: is prairie agriculture becoming like any other industry? Does it matter? In: Canadian journal of agricultural economics, Band 48 (2000), Heft 4, S. 385-395.

Stiglitz, J. E. (2002): Information and the Change in the Paradigm in Economics. In: American Economic Review, Band 92, Heft 3, S. 460-501.

Stiglitz, J. E. (1987): The causes and consequences of the dependence of quality on price. In: Journal of Economic Literature, Band 25, Heft 1, S. 1-48.

Stouffer, S. A. und Osborn, F. (1949): The American soldier: combat and its aftermath; Princeton.

Tanneberger, T. und Jaster, K. (2006): Wieviel Management braucht der Betrieb? In: Neue Landwirtschaft, 11/2006, S. 14-18.

Tanneberger, T. (1997): Vergütungssysteme in der Praxis. In: Neue Landwirtschaft, 5/1997, S. 18-21.

Theuvsen, L. (2003): Erfolgsbedingungen leistungsorientierter Entgeltsysteme. In: Die Verwaltung, Band 36, Heft 4, S. 483-500.

Tolstoj, L.: Anna Karenina, 1. Auflage, Insel-Taschenbuch-Verlag; Frankfurt am Main.

Wiener, B. et al. (2004): Abschätzung des Bedarfs landwirtschaftlicher Fachkräfte unter Berücksichtigung der demografischen Entwicklung (Schwerpunkt neue Bundesländer), Bericht Nr. 04-2, Zentrum für Sozialforschung Halle e.V..

Wolff, B. und Lazear, E. P. (2001): Einführung in die Personalökonomik; Stuttgart. 
Wüllmers, L. (1995): Motivationsstrategien in landwirtschaftlichen Großunternehmen mit Lohnarbeitsverfassung - eine empirische Untersuchung in Sachsen und Thüringen, Diplomarbeit, Universität Göttingen.

Yellen, J. L. (1984): Efficiency wage of models of unemployment. In: American Economic Review, Band 74, Heft 2, S. 200-205.

Ziron, M. (2007): Erfolgsfaktoren von Spitzenbetrieben in der Schweineproduktion, Folienvortrag vom 10.1.2007, DLG-Wintertagung 2007 in München Download vom 06.05.2007 unter http://download.dlg.org/pdf/wita2007/PR_Ziron.pdf.

\section{Mündliche und schriftliche Auskünfte:}

Betriebsleiter A: Experteninterview vom 01.12.2006 mit dem Geschäftsführer einer Agrargenossenschaft in Sachsen-Anhalt.

Betriebsleiter B: Wortbeitrag des Geschäftsführers einer Agrargenossenschaft in Thüringen während der Ergebnisdiskussion

Betriebsleiter C: Schriftliche Anmerkung im Betriebsleiterfragebogen.

Betriebsleiter D: Schriftliche Anmerkung im Betriebsleiterfragebogen.

Fechner (2006): Telefonische Auskunft vom 01.12.2006 von Dr. Jürgen Fechner, Fachgebietsleiter Agrarökonomie, Landesamt für Verbraucherschutz, Landwirtschaft und Flurneuordnung des Landes Brandenburg.

Heil (2007): Schriftliche Auskunft vom 19.4.2007 von Sigrun Heil, Pressesprecherin der IG Bauen-Agrar-Umwelt.

Weidel (2006): Schriftliche Auskunft vom 10.2.2006 von Hans-Peter Weidel, Regionalsekretär für die Regionen Nord und Niedersachsen-Bremen der IG Bauen-Agrar-Umwelt. 


\section{Verzeichnis der verwendeten Rechtsquellen:}

Arbeitszeitgesetz (ArbZG): Arbeitszeitgesetz vom 06.06.1994 (BGBl. I, S. 1170/1171) zuletzt geändert durch Artikel 229 der Verordnung vom 31.10.2006 (BGBl. I S. 2407)

Betriebsverfassungsgesetz (BetrVG): Betriebsverfassungsgesetz vom 15.01.1972 (BGBl. I, S. 13), zuletzt geändert durch Artikel 221 der Verordnung vom 31.10.2006 (BGB1. I, S. 2407, S. 2434)

Bürgerliches Gesetzbuch (BGB): In der Fassung vom 02.01.2002 (BGBl. I, S. 42), zuletzt geändert durch das Gesetz vom 22.12.2006 (BGB1. I, S. 3416)

Drittelbeteiligungsgesetz (DrittelbG): Gesetz über die Drittelbeteiligung der Arbeitnehmer im Aufsichtsrat vom 18.05.2004 (BGB1. 2004, S. 974), zuletzt geändert durch Artikel 19 des Gesetze zur Einführung der Europäischen Genossenschaft und zur Änderung des Genossenschaftsrechts vom 14.08.2006 (BGB1. 2006 I., S. 1911)

Entgelttarifvertrag für die Beschäftigten der Rinderzucht Schleswig-Holstein eG vom 16.01.2002.

Erneuerbare-Energien-Gesetz (EEG): Gesetz zur Neuregelung des Rechts der Erneuerbaren Energien im Strombereich vom 21.07. 2004 (BGB1. I, S. 1918)

Tarifvertragsgesetz (TVG): Tarifvertragsgesetz in der Fassung der Bekanntmachung vom 25.08.1969 (BGB1. I, S. 1323), zuletzt geändert durch Artikel 223 der Verordnung vom 31.10.2006 (BGB1. I S. 2407)

Tarifvertrag über die Vergütung von Außendienstmitarbeitern der Rinderzucht Schleswig-Holstein eG vom 16.06.2003

Teilzeit- und Befristungsgesetz (TzBfG): Teilzeit- und Befristungsgesetz vom 21.12.2000 (BGBl. I, S. 1966), zuletzt geändert durch Artikel 2 des Gesetzes vom 24.12.2003 (BGB1. I, S. 3002)

Lohntarifvertrag vom 01.01.2000 für die Melker in Schleswig-Holstein

Lohntarifvertrag für die Landarbeiter in Schleswig-Holstein vom 22.04.2006 


\section{Anhang Tabellenband}

\section{Georg-August-Universität Göttingen}

Arbeitsbereich Angewandte landwirtschaftliche Betriebslehre

Prof. Dr. Enno Bahrs

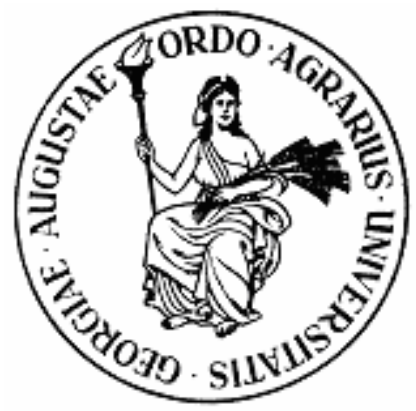

Ergebnisband zum Forschungsprojekt „Anreizsysteme für Fremdarbeitskräfte in der Landwirtschaft - eine empirische Analyse“"

M. Sc. Zazie v. Davier

Department für Agrarökonomie und Rurale Entwicklung

Platz der Göttinger Sieben 5

37073 Göttingen

Email: zdavier@uni-goettingen.de 


\section{TEIL I \\ Ergebnisse der Betriebsleiterbefragung}




\section{Ausgewählte Ergebnisse der Betriebsleiterbefragung in Kürze:}

- 260 Betriebsleiter aus verschiedenen Bundesländern; Schwerpunkt Mecklenburg-Vorpommern, Thüringen, Schleswig-Holstein, Niedersachsen

- Durchschnittlich haben die Betriebe 894,1 ha Ackerfläche, 2.599 Mastschweine- und 715 Zuchtsauenplätze, halten 340 Milchkühe und 373 Rinder.

- In den Betrieben sind durchschnittlich 2,15 Familienarbeitskräfte und 15,43 Lohnarbeitskräfte beschäftigt.

- Die Betriebsleiter sind überwiegend sehr zufrieden mit dem Arbeitseinsatz und der Motivation ihrer Mitarbeiter.

- Die Betriebsleiter entlohnen ihre Mitarbeiter vorwiegend durch einen festen Stundenlohn oder durch ein Gehalt.

- Die durchschnittlichen Bruttolöhne liegen in der Pflanzenproduktion bei 8,54 Euro/h, in der Tierproduktion bei 8,19 Euro/h. Das Lohngefälle zwischen den alten und neuen Bundesländern beträgt in der Produktion circa 2,50 Euro.

$>$ Das Lohnniveau in der Landwirtschaft ist im Vergleich zu anderen Branchen niedrig.

- Leistungs- und erfolgsabhängige Vergütungen spielen in der Tierproduktion eine größere Rolle als im Ackerbau.

- Leistungsabhängige Vergütung sollte möglichst auf objektiv messbaren Kriterien beruhen, dies ist im Ackerbau besonders schwierig.

- Der variable Vergütungsanteil liegt durchschnittlich bei $14,83 \%$.

$>$ Der variable Anteil sollte 10-30 \% betragen, damit die Mitarbeiter den Leistungslohn überhaupt wahrnehmen.

- Die Betriebsleiter verwenden unterschiedliche subjektive und objektive Leistungsindikatoren.

$>$ Leistungslöhne sollten auf möglichst wenigen (max. 4-5) Parametern beruhen, damit der Ursache-Wirkungszusammenhang für die Mitarbeiter überschaubar bleibt

- Leistungsindikatoren sollten im Einflussbereich der Mitarbeiter liegen, um eine Motivationswirkung zu erzielen.

- Die Erfahrungen der Betriebsleiter mit Leistungslöhnen sind unterschiedlich.

- Die Leiter der ökologisch wirtschaftenden Betriebe bestätigen zwar ihre Motivationswirkung, sehen aber Leistungslöhne in Bezug auf mögliche Nachteile am kritischsten.

- Die Betriebsleiter der juristischen Personen, die über die meiste Erfahrung mit Leistungslöhnen verfügen, stimmen einer positiven Motivationswirkung nur zum Teil zu. 


\section{Häufigkeitsauszählungen und Mittelwerte des Betriebsleiterfragebogens}

1. Wie viel Fläche in ha LF bewirtschaften Sie?

\begin{tabular}{|l|c|c|}
\hline Flächenart: & Nennungen & ha (Mittelwert) \\
\hline Ackerland: & $\mathrm{n}=256$ & 894,17 \\
\hline Grünland: & $\mathrm{n}=221$ & 221,87 \\
\hline Wald: & $\mathrm{n}=135$ & 38,79 \\
\hline Gesamt & $\mathrm{n}=257$ & 1097,57 \\
\hline
\end{tabular}

2. Wie setzt sich der Eigentums- und Pachtanteil an Boden in Ihrem Betrieb zusammen?

\begin{tabular}{|l|c|c|}
\hline Boden: & Nennungen & ha (Mittelwert) \\
\hline Fläche insgesamt: & $\mathrm{n}=254$ & 1073,17 \\
\hline Davon Eigentumsfläche: & $\mathrm{n}=243$ & 257,73 \\
\hline Davon Pachtfläche: & $\mathrm{n}=243$ & 835,43 \\
\hline
\end{tabular}

\section{Wie viel Vieh halten Sie im Jahresdurchschnitt?}

\begin{tabular}{|l|c|c|}
\hline Tierart: & Nennungen: & Anzahl: Mittelwert \\
\hline Milchkühe: & $\mathrm{n}=143$ & 340 \\
\hline Nachzucht (Rinder): & $\mathrm{n}=159$ & 373 \\
\hline Bullen/Mastrinder: & $\mathrm{n}=77$ & 199 \\
\hline Mutterkühe: & $\mathrm{n}=40$ & 233 \\
\hline Mutterschafe: & $\mathrm{n}=7$ & 475 \\
\hline & & Plätze: Mittelwert \\
\hline Mastschweine: & $\mathrm{n}=65$ & 2599 \\
\hline Sauen: & $\mathrm{n}=46$ & 715 \\
\hline Ferkelaufzucht: & $\mathrm{n}=7$ & 1194 \\
\hline
\end{tabular}




\section{Nehmen Sie Beratung in Anspruch? Wenn ja, um welche Art von Beratung} handelt es sich (Mehrfachantworten sind möglich)? $\mathbf{n = 2 6 0}$

$3,5 \%$ Nein

$96,2 \% \mathrm{Ja}$, und zwar durch:

$0,3 \%$ keine Angabe

\begin{tabular}{|l|c|c|c|c|}
\hline Beratungsträger: & \multicolumn{3}{|c|}{ Beratung auf dem Gebiet der ... } \\
\hline & $\begin{array}{c}\text { Pflanzen- } \\
\text { produktion }\end{array}$ & $\begin{array}{c}\text { Tier- } \\
\text { produktion }\end{array}$ & $\begin{array}{c}\text { Betriebs- } \\
\text { wirtschaft }\end{array}$ & $\begin{array}{c}\text { Sonstige } \\
\text { z.B. } \\
\text { Steuerberatung, } \\
\text { Anträge, Biogas }\end{array}$ \\
\hline $\begin{array}{l}\text { Landwirtschaftskammer/-ämter } \\
\text { Pflanzenschutzamt }\end{array}$ & $54,2 \%$ & $18,1 \%$ & $16,9 \%$ & $5,4 \%$ \\
\hline Private Beratungsbüros & $24,2 \%$ & $18,8 \%$ & $33,5 \%$ & $6,2 \%$ \\
\hline Ringberatung & $19,6 \%$ & $21,9 \%$ & $17,7 \%$ & $2,3 \%$ \\
\hline Firmenberater & $43,1 \%$ & $33,5 \%$ & $6,5 \%$ & $3,1 \%$ \\
\hline Andere Landwirte Genossenschafts- & $24,6 \%$ & $19,2 \%$ & $8,1 \%$ & $4,6 \%$ \\
\hline $\begin{array}{l}\text { Sonstige (u.a. Gerband, LMS, Anbauverbände, } \\
\text { verband, } \\
\text { Bauernverband) }\end{array}$ & $5,4 \%$ & $6,9 \%$ & $13,1 \%$ & $4,6 \%$ \\
\hline
\end{tabular}

5. Bitte geben Sie an, inwieweit die folgenden Aussagen auf Sie persönlich zutreffen. $\mathbf{n}=\mathbf{2 6 0}$

\begin{tabular}{|c|c|c|c|c|c|c|}
\hline & $\begin{array}{l}\text { Stimme } \\
\text { voll und } \\
\text { ganz zu }\end{array}$ & $\begin{array}{l}\text { Stimme } \\
\mathrm{zu}\end{array}$ & $\begin{array}{l}\text { Teils/ } \\
\text { teils }\end{array}$ & $\begin{array}{l}\text { Lehne } \\
\mathrm{ab}\end{array}$ & $\begin{array}{l}\text { Lehne } \\
\text { voll } \\
\text { und } \\
\text { ganz ab }\end{array}$ & $\begin{array}{l}\text { Mittel- } \\
\text { wert }\end{array}$ \\
\hline $\begin{array}{l}\text { Für mich ist die Zeit am } \\
\text { Schreibtisch genauso sinnvoll } \\
\text { wie die tägliche Arbeit im } \\
\text { Betrieb. }\end{array}$ & $48,1 \%$ & $31,2 \%$ & $17,3 \%$ & $1,5 \%$ & $0,4 \%$ & 1,27 \\
\hline $\begin{array}{l}\text { Ich riskiere auch mal Dinge, die } \\
\text { eventuell scheitern können. }\end{array}$ & $11,2 \%$ & $31,5 \%$ & $39,6 \%$ & $13,8 \%$ & $1,2 \%$ & 0,39 \\
\hline $\begin{array}{l}\text { Ich bin an technologischen } \\
\text { Neuerungen in meinem Betrieb } \\
\text { sehr interessiert. }\end{array}$ & $29,6 \%$ & $51,9 \%$ & $16,5 \%$ & $0,4 \%$ & & 1,13 \\
\hline $\begin{array}{l}\text { Jeder Landwirt sollte seine } \\
\text { betriebswirtschaftlichen } \\
\text { Kennzahlen aus dem Stehgreif } \\
\text { kennen. }\end{array}$ & $35,4 \%$ & $46,9 \%$ & $14,6 \%$ & $1,9 \%$ & & 1,17 \\
\hline $\begin{array}{l}\text { Ich bin draußen effektiver als am } \\
\text { Schreibtisch. }\end{array}$ & $3,8 \%$ & $13,1 \%$ & $45,0 \%$ & $16,9 \%$ & $10,0 \%$ & $-0,06$ \\
\hline $\begin{array}{l}\text { Um meine Zukunft mache ich } \\
\text { mir keine Sorgen, da sich gute } \\
\text { Unternehmen durchsetzen } \\
\text { werden. }\end{array}$ & $14,6 \%$ & $36,9 \%$ & $37,7 \%$ & $8,1 \%$ & $1,5 \%$ & 0,56 \\
\hline $\begin{array}{l}\text { Ich verbringe sehr viel Zeit mit } \\
\text { der zukünftigen Planung und } \\
\text { Ausrichtung meines Betriebes. }\end{array}$ & $13,8 \%$ & $49,2 \%$ & $32,7 \%$ & $3,1 \%$ & $0,4 \%$ & 0,74 \\
\hline
\end{tabular}


6. Wie schätzen Sie die Zukunft Ihres landwirtschaftlichen Betriebes ein? n=260

\begin{tabular}{|l|c|c|c|c|c|c|}
\hline In Bezug auf... & $\begin{array}{c}\text { sehr opti- } \\
\text { mistisch }\end{array}$ & $\begin{array}{c}\text { opti- } \\
\text { mistisch }\end{array}$ & $\begin{array}{c}\text { weder } \\
\text { optimistisch } \\
\text { noch } \\
\text { pessimistisch }\end{array}$ & $\begin{array}{c}\text { pessi- } \\
\text { mistisch }\end{array}$ & $\begin{array}{c}\text { sehr } \\
\text { pessi- } \\
\text { mistisch }\end{array}$ & $\begin{array}{c}\text { Mittel- } \\
\text { wert }\end{array}$ \\
\hline $\begin{array}{l}\text { W. die allgemeine } \\
\text { Ihettbewerbsfähigkeit }\end{array}$ & $6,2 \%$ & $65,4 \%$ & $22,7 \%$ & $4,3 \%$ & $0,8 \%$ & 0,67 \\
\hline $\begin{array}{l}\ldots \text { die Wachstumsmög- } \\
\text { lichkeiten Ihres } \\
\text { Betriebes. }\end{array}$ & $3,1 \%$ & $31,9 \%$ & $48,8 \%$ & $14,6 \%$ & $0,8 \%$ & 0,22 \\
\hline $\begin{array}{l}\ldots \text { den wirtschaftlichen } \\
\text { Erfolg Ihres Betriebes. }\end{array}$ & $1,9 \%$ & $60,0 \%$ & $31,2 \%$ & $6,2 \%$ & $0 \%$ & 0,58 \\
\hline $\begin{array}{l}\text { Bis zu 100\% fehlende Prozente: Keine Angaben } \\
\text { Mittelwertberechnung: hier sind die Betriebsleiter herausgerechnet, die keine Angaben gemacht haben, } \\
\text { Skalierung von +2, sehr optimistisch“ bis -2, ,sehr pessimistisch“ }\end{array}$ \\
\hline
\end{tabular}

7. Wie viele Familienangehörige und Fremdarbeitskräfte arbeiten auf Ihrem Betrieb? (Bitte die Anzahl an Personen angeben!)

\begin{tabular}{|l|l|l|l|}
\hline Art der Arbeitskräfte & \multicolumn{1}{|c|}{ Insgesamt } & \multicolumn{1}{|c|}{$\begin{array}{c}\text { Davon } \\
\text { Vollzeit }\end{array}$} & $\begin{array}{c}\text { Davon } \\
\text { Teilzeit }\end{array}$ \\
\hline Familienarbeitskräfte (Anzahl Personen) & $2,15(\mathrm{n}=148)$ & $1,77(\mathrm{n}=132)$ & $1,12(\mathrm{n}=74)$ \\
\hline Fremdarbeitskräfte (Anzahl Personen) & $15,47(\mathrm{n}=249)$ & $13,63(\mathrm{n}=245)$ & $3,96(\mathrm{n}=127)$ \\
\hline Davon Auszubildende & $2,69(\mathrm{n}=168)$ & $2,73(\mathrm{n}=163)$ & $1,875(\mathrm{n}=4)$ \\
\hline
\end{tabular}

8. Wie verteilen sich prozentual Ihre Arbeitsschwerpunkte im Betrieb? Bitte geben Sie den prozentualen Wert so an, dass die Summe $100 \%$ ergibt.

\begin{tabular}{|l|c|}
\hline Betriebsbereich: & Prozent (Mittelwerte) \\
\hline Produktionstechnik - Pflanze & 34,68 \\
\hline Produktionstechnik - Tier & 37,40 \\
\hline Verwaltungstätigkeiten (Büro, Einkauf, Betriebsorganisation) & 30,62 \\
\hline Sonstiges......................................... & 15,59 \\
\hline Sonstiges...................................... & 13,74 \\
\hline
\end{tabular}

9. Wie schätzen Sie den zukünftigen Fachkräftebedarf in der Landwirtschaft für Ihre Region ein? n=260*, *Bis zu 100\% fehlende Prozente: Keine Angaben

$\begin{array}{ccccc}\text { stark sinkend } & \text { etwas sinkend } & \text { gleich bleibend } & \text { etwas steigend } & \text { stark steigend } \\ 4,2 \% & 33,5 \% & 28,8 \% & 26,5 \% & 5,4 \%\end{array}$


10. Wie schätzen Sie den zukünftigen Fachkräftebedarf in der Landwirtschaft für Ihren Betrieb ein? $n=260$ *

$\begin{array}{ccccc}\text { stark sinkend } & \text { etwas sinkend } & \text { gleich bleibend } & \text { etwas steigend } & \text { stark steigend } \\ 1,2 \% & 26,5 \% & 45,0 \% & 20,8 \% & 4,2 \%\end{array}$

*Bis zu 100\% fehlende Prozente: Keine Angaben

11. Wie hoch ist der Bruttostundenlohn (Arbeitnehmerbrutto) Ihrer Mitarbeiter?

\begin{tabular}{|l|c|c|c|c|}
\hline Bereich & $\begin{array}{c}\varnothing \text { Bruttostundenlohn } \\
\text { (ohne Zuschläge) in } \\
€ / \mathrm{h}\end{array}$ & $\begin{array}{c}\varnothing \\
\text { Bruttostundenlohn } \\
\text { (mit Zuschlägen) in } \\
€ / \mathrm{h}\end{array}$ & $\begin{array}{c}\varnothing \\
\text { Bruttostundenlohn } \\
\text { (ohne Zuschläge) in } \\
€ / \mathrm{h} \mathrm{ABL}\end{array}$ & $\begin{array}{c}\varnothing \text { Bruttostunden- } \\
\text { lohn (ohne } \\
\text { Zuschläge) in } € / \mathrm{h} \\
\text { NBL }\end{array}$ \\
\hline Leitung & 14,59 & 15,72 & 16,87 & 14,27 \\
\hline Verwaltung & 9,62 & 10,38 & 9,7 & 9,63 \\
\hline $\begin{array}{l}\text { Pflanzen- } \\
\text { produktion }\end{array}$ & 8,54 & 9,24 & 10,87 & 8,02 \\
\hline $\begin{array}{l}\text { Tier- } \\
\text { produktion }\end{array}$ & 8,19 & 8,79 & 10,21 & 7,81 \\
\hline Sonstiges & & & & \\
\hline
\end{tabular}

\section{Die folgende Frage bezieht sich auf die Motivation Ihrer Mitarbeiter. Stimmen Sie} diesen Aussagen zu oder lehnen Sie diese ab? $\mathbf{n}=\mathbf{2 6 0}$

\begin{tabular}{|c|c|c|c|c|c|c|}
\hline & $\begin{array}{l}\text { Stimme } \\
\text { voll und } \\
\text { ganz zu }\end{array}$ & $\begin{array}{c}\text { Stimme } \\
\mathrm{zu}\end{array}$ & $\begin{array}{l}\text { Teils/ } \\
\text { teils }\end{array}$ & $\begin{array}{c}\text { Lehne } \\
\text { ab }\end{array}$ & $\begin{array}{c}\text { Lehne voll } \\
\text { und ganz } \\
\text { ab }\end{array}$ & Mittelwert \\
\hline $\begin{array}{l}\text { Die Mitarbeiter in meinem } \\
\text { Betrieb erledigen Ihre Arbeit } \\
\text { gewissenhaft und } \mathrm{zu} \text { meiner } \\
\text { vollsten Zufriedenheit. }\end{array}$ & $13,5 \%$ & $53,5 \%$ & $31,5 \%$ & & & 0,82 \\
\hline $\begin{array}{l}\text { Meinen Mitarbeitern geht es } \\
\text { nicht nur ums Geld, ihnen } \\
\text { macht die Arbeit Spaß. }\end{array}$ & $11,2 \%$ & $47,3 \%$ & $37,3 \%$ & $1,9 \%$ & & 0,69 \\
\hline $\begin{array}{l}\text { Meine Mitarbeiter hängen an } \\
\text { der Arbeit in der } \\
\text { Landwirtschaft. }\end{array}$ & $14,2 \%$ & $56,2 \%$ & $26,2 \%$ & $1,5 \%$ & & 0,85 \\
\hline $\begin{array}{l}\text { Meine Mitarbeiter sind bereit } \\
\text { auch mal länger zu arbeiten. }\end{array}$ & $51,4 \%$ & $44,4 \%$ & $2,7 \%$ & $0,4 \%$ & & 1,47 \\
\hline $\begin{array}{l}\text { Meine Mitarbeiter erledigen } \\
\text { ihre Aufgaben selbstständig. }\end{array}$ & $15,0 \%$ & $46,5 \%$ & $36,2 \%$ & $0,8 \%$ & & 0,77 \\
\hline
\end{tabular}


13. Wie beurteilen Sie die folgenden Aussagen zu Ihren Unternehmenszielen? Bitte geben Sie wieder den Grad Ihrer Zustimmung bzw. Ablehnung an! n=260

\begin{tabular}{|c|c|c|c|c|c|c|}
\hline & $\begin{array}{l}\text { Stimme } \\
\text { voll und } \\
\text { ganz zu }\end{array}$ & $\begin{array}{c}\text { Stimme } \\
\mathrm{zu}\end{array}$ & $\begin{array}{l}\text { Teils/ } \\
\text { teils }\end{array}$ & $\begin{array}{l}\text { Lehne } \\
\text { ab }\end{array}$ & $\begin{array}{l}\text { Lehne } \\
\text { voll } \\
\text { und ganz } \\
\text { ab }\end{array}$ & $\begin{array}{l}\text { Mittel- } \\
\text { wert }\end{array}$ \\
\hline $\begin{array}{l}\text { Ich spreche regelmäßig } \\
\text { mit meinen Mitarbeitern } \\
\text { über die Ziele des } \\
\text { Unternehmens. }\end{array}$ & $12,3 \%$ & $53,1 \%$ & $29,6 \%$ & $3,5 \%$ & & 0,75 \\
\hline $\begin{array}{l}\text { Meine Mitarbeiter } \\
\text { können die Ziele des } \\
\text { Unternehmens klar } \\
\text { benennen. }\end{array}$ & $3,5 \%$ & $31,9 \%$ & $58,1 \%$ & $4,6 \%$ & $0,4 \%$ & 0,34 \\
\hline $\begin{array}{l}\text { Nur wenige Mitarbeiter } \\
\text { überschauen die } \\
\text { wirtschaftlichen } \\
\text { Zusammenhänge im } \\
\text { Unternehmen. }\end{array}$ & $1,5 \%$ & $10,4 \%$ & $38,5 \%$ & $39,6 \%$ & $8,5 \%$ & 0,45 \\
\hline $\begin{array}{l}\text { Jeder Mitarbeiter weiß, } \\
\text { wie er zur Realisierung } \\
\text { der Unternehmensziele } \\
\text { beitragen kann. }\end{array}$ & $4,2 \%$ & $49,6 \%$ & $39,2 \%$ & $5,0 \%$ & $0,4 \%$ & 0,54 \\
\hline $\begin{array}{l}\text { Ich setze Ziele so, dass } \\
\text { meine Mitarbeiter sie } \\
\text { möglichst erreichen } \\
\text { können. }\end{array}$ & $12,7 \%$ & $71,2 \%$ & $13,5 \%$ & $1,2 \%$ & & 0,97 \\
\hline $\begin{array}{l}\mathrm{Zu} \text { viele Ziele verwirren } \\
\text { meine Mitarbeiter. }\end{array}$ & $2,3 \%$ & $9,6 \%$ & $23,1 \%$ & $43,5 \%$ & $18,8 \%$ & $-0,69$ \\
\hline
\end{tabular}


14. Die nächsten Fragen beziehen sich auf das in Ihrem Betrieb angewendete Vergütungssystem. Bitte füllen Sie nur die Teilfragen aus, die sich auf Ihre Betriebszweige beziehen. Mehrfachantworten sind möglich.

a)

Wie werden die Mitarbeiter im Ackerbau derzeit entlohnt? n=241

\begin{tabular}{|l|c|}
\hline Fester Stundenlohn/Gehalt & $94,2 \%$ \\
\hline Höhergruppierung in Lohnstufen in Abhängigkeit von der Leistung & $26,6 \%$ \\
\hline Zuschläge für Überstunden & $35,3 \%$ \\
\hline Zuschläge für Sonn- und Feiertagsarbeit & $56,4 \%$ \\
\hline Zulagen für Arbeitserschwernis (z.B. Pflanzenschutzmaßnahmen) & $17,9 \%$ \\
\hline $\begin{array}{l}\text { Sie erhalten Leistungszulagen (z.B. für sorgfältigen Umgang mit Maschinen, } \\
\text { Arbeitseinsatz), wenn ich mit ihnen zufrieden bin. }\end{array}$ & $15,4 \%$ \\
\hline Sie erhalten Prämien, wenn sie bestimmte Leistungskriterien erfüllen. & $16,2 \%$ \\
\hline Erfolgsbeteiligungen & $17,4 \%$ \\
\hline $\begin{array}{l}\text { Sonstige: 1000€/Jahr Prämie, davon werden durch Nachlässigkeit verschuldete Schäden abgezogen } \\
\text { (außerhalb des Lohns), 40€ Werkzeuggeld bei vorhandener Grundausstattung, Beiträge zur } \\
\text { Altersvorsorgung in Abhängigkeit vom Betriebsergebnis, Ernteprämie, Weihnachtsgeld, Bei } \\
\text { Unterschreitung eines gewissen Budgets für Unterhaltung von Maschinen und Geräten erhalten die } \\
\text { Mitarbeiter einen Anteil davon, Zu- und Abschläge für besonderen Einsatz, Zuzahlung bei Arbeit und } \\
\text { Krankheit und Unfall }\end{array}$ \\
\hline
\end{tabular}

Wie werden die Mitarbeiter in der Milchproduktion derzeit entlohnt? $n=139$

\begin{tabular}{|l|c|}
\hline Fester Stundenlohn/Gehalt & $92,1 \%$ \\
\hline Höhergruppierung in Lohnstufen in Abhängigkeit von der Leistung & $27,3 \%$ \\
\hline Zuschläge für Überstunden & $23,7 \%$ \\
\hline Zuschläge für Sonn- und Feiertagsarbeit & $59,7 \%$ \\
\hline $\begin{array}{l}\text { Sie erhalten Leistungszulagen (z.B. für sorgfältigen Umgang mit Maschinen, } \\
\text { Arbeitseinsatz), wenn ich mit ihnen zufrieden bin. }\end{array}$ & $16,5 \%$ \\
\hline Sie erhalten Prämien, wenn sie bestimmte Leistungskriterien erfüllen. & $36,0 \%$ \\
\hline Erfolgsbeteiligungen & $23,2 \%$ \\
\hline $\begin{array}{l}\text { Sonstige: 100 \% Leistungsvergütung als Teamvergütung, 4,5 Ct/kg Milch, verteilt auf alle } \\
\text { Beschäftigten in der Milchproduktion, eventuell Beiträge zur Altersvorsorgung in Abhängigkeit vom } \\
\text { Betriebsergebnis, Grundlohn plus 1€ Zuschlag max. der sich aus Totgeburten, Milchmenge, S- } \\
\text { Qualität und Tierarztkosten zusammensetzt; Gruppenprämie 0,5€/l ermolkene Milch/Monat bei } \\
\text { entsprechender Qualität - Aufteilung nach geleisteten Arbeitsstunden (ohne Krankheit und Urlaub); } \\
\text { Zuzahlung bei Arbeit und Krankheit und Unfall, Weihnachtsgeld }\end{array}$ \\
\hline
\end{tabular}


c) Wie werden die Mitarbeiter in der Schweineproduktion derzeit entlohnt? $\quad n=70$

\begin{tabular}{|l|c|}
\hline Fester Stundenlohn/Gehalt & $91,4 \%$ \\
\hline Höhergruppierung in Lohnstufen in Abhängigkeit von der Leistung & $20,0 \%$ \\
\hline Zuschläge für Überstunden & $27,1 \%$ \\
\hline Zuschläge für Sonn- und Feiertagsarbeit & $52,9 \%$ \\
\hline $\begin{array}{l}\text { Sie erhalten Leistungszulagen (z.B. für sorgfältigen Umgang mit Maschinen, } \\
\text { Arbeitseinsatz), wenn ich mit ihnen zufrieden bin. }\end{array}$ & $7,2 \%$ \\
\hline Sie erhalten Prämien, wenn sie bestimmte Leistungskriterien erfüllen. & $24,3 \%$ \\
\hline Erfolgsbeteiligungen & $26,1 \%$ \\
\hline $\begin{array}{l}\text { Sonstige: 100\% Leistungsvergütung als Teamvergütung in Sauenanlage und Flatdeck, Vergütung nach } \\
\text { Endprodukt (Teamvergütung), Zunahmen pro Tag nach ausgestalten Abteilungen }\end{array}$ \\
\hline
\end{tabular}

d) Falls es in Ihrem Betrieb Führungskräfte gibt (z.B. Verwalter) welche Vergütungsregelungen wurden für diese gefunden? $n=123$

\begin{tabular}{|c|c|}
\hline Fester Stundenlohn/Gehalt & $96,7 \%$ \\
\hline Höhergruppierung in Lohnstufen in Abhängigkeit von der Leistung & $12,2 \%$ \\
\hline Tantiemeregelung & $21,1 \%$ \\
\hline Gewinnbeteiligung & $24,4 \%$ \\
\hline Kapitalbeteiligung & $4,1 \%$ \\
\hline Prämien, z.B. für die Entwicklung der Kosten oder der Milchleistung & $11,4 \%$ \\
\hline \multicolumn{2}{|c|}{$\begin{array}{l}\text { Sonstige: eventuell Beiträge zur Altersvorsorgung in Abhängigkeit vom Betriebsergebnis, bei } \\
\text {,normalen Arbeiten“ z.B. Güllefahren liegt der Lohn 1,5€ über dem normalen Arbeiter, Gehalts- } \\
\text { anhebung nach Betriebsentwicklung (subjektiv), ergebnisabhängige Sonderzahlungen, Betriebsrente, } \\
\text { innerhalb der KG ist der Lohn mehrheitlich gewinnbeteiligt, Erfolgbeteiligung am Ende des } \\
\text { Wirtschaftsjahres, Weihnachtsgratifikation }\end{array}$} \\
\hline
\end{tabular}

\section{Haben Sie bereits Erfahrungen mit leistungsorientierten Vergütungssystemen gesammelt?}

\begin{tabular}{|l|c|c|c|}
\hline & Gesamt & alte Bundesländer & neue Bundesländer \\
\hline Ja & $42,9 \%(\mathrm{n}=111)$ & $27,1 \%(\mathrm{n}=16)$ & $47,3 \%(\mathrm{n}=89)$ \\
\hline Nein & $57,1 \%(\mathrm{n}=148)$ & $72,9 \%(\mathrm{n}=43)$ & $52,7 \%(\mathrm{n}=95)$ \\
\hline
\end{tabular}

Wenn ja, dann weiter mit Frage 16

Wenn nein, dann weiter mit Frage 19 


\section{6. a) An welchen Kriterien machen Sie die leistungsorientierte Vergütung Ihrer} Mitarbeiter im Ackerbau fest? (Mehrfachantworten möglich) $n=62$

\begin{tabular}{|l|c|}
\hline Arbeitsleistung (z.B. Druschleistung, gepflügte Fläche/h) & $48,4 \%$ \\
\hline Sorgfältiger Umgang mit Maschinen & $35,5 \%$ \\
\hline Maschinenkosten & $22,6 \%$ \\
\hline Reparaturkosten & $29 \%$ \\
\hline Deckungsbeitrag des Betriebszweigs & $25,8 \%$ \\
\hline Hektarerträge & $21 \%$ \\
\hline Sparsamer Einsatz von Pflanzenschutzmitteln & $1,6 \%$ \\
\hline $\begin{array}{l}\text { Sonstige: Prämie für Silagequalität, Anerkennung der Vermehrungskartoffeln, sparsamer Einsatz von } \\
\text { Diesel, Reinertrag Acker, Qualifikation / Geschwindigkeit /persönliche Fähigkeiten, technische } \\
\text { Erfindungen, wenig Krankheitstage und Unfälle, Werkzeuggeld, agrotechnische Termine }\end{array}$
\end{tabular}

b) An welchen Kriterien machen Sie die leistungsorientierte Vergütung Ihrer Mitarbeiter in der Milchproduktion fest? (Mehrfachantworten möglich) n=67

\begin{tabular}{|l|c|}
\hline Milchleistung & $65,7 \%$ \\
\hline Milchqualität & $77,6 \%$ \\
\hline Reproduktionsrate & $20,9 \%$ \\
\hline Tierarztkosten & $14,9 \%$ \\
\hline Zwischenkalbezeit & $11,9 \%$ \\
\hline Zellzahlen & $47 \%$ \\
\hline Deckungsbeitrag des Betriebszweigs & $9 \%$ \\
\hline Grundfutterleistung & $1,5 \%$ \\
\hline Kälberverluste & $43,3 \%$ \\
\hline $\begin{array}{l}\text { Sonstige: Besamungsindex, Ordnung und Sauberkeit, Brunstbeobachtung, Gesamtergebnis Betrieb, } \\
\text { Höhe des monatlichen Milchgeldes, Qualität der Fütterung gemessen am Harnstoffwert, saubere } \\
\text { Siloanschnittflächen, Trächtigkeitsrate }\end{array}$ \\
\hline
\end{tabular}

c) An welchen Kriterien machen Sie die leistungsorientierte Vergütung Ihrer Mitarbeiter in der Schweineproduktion fest? (Mehrfachantworten möglich) $\mathbf{n}=\mathbf{2 4}$

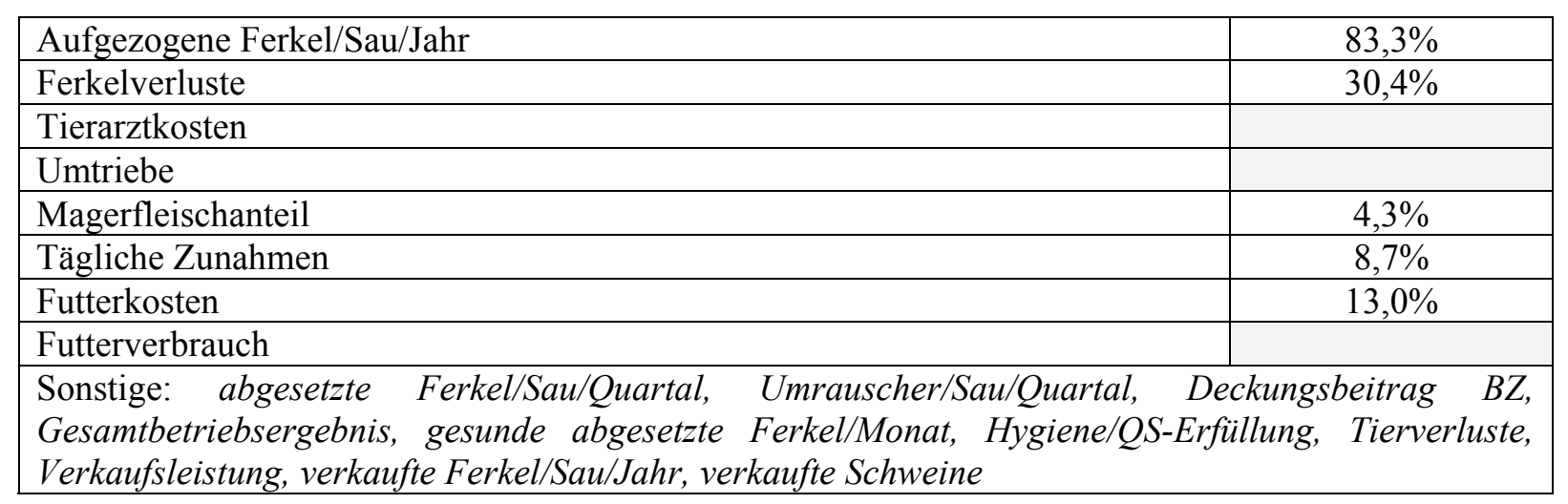


17. Wie hoch ist der Anteil der leistungsabhängigen Entlohnung am Gesamtlohn? $\mathrm{n}=72$

Mittelwert: $14,83 \% \quad$ Minimum: $1 \% \quad$ Maximum: $70 \%$

18. Wenn Sie an Ihre Erfahrungen mit leistungsorientierten Vergütungssystemen denken, inwieweit stimmen Sie den folgenden Aussagen zu? n=104

\begin{tabular}{|c|c|c|c|c|c|c|}
\hline & $\begin{array}{l}\text { Stimme } \\
\text { voll und } \\
\text { ganz zu }\end{array}$ & $\begin{array}{l}\text { Stimme } \\
\mathrm{zu}\end{array}$ & $\begin{array}{l}\text { Teils/ } \\
\text { teils }\end{array}$ & $\begin{array}{l}\text { Lehne } \\
\mathrm{ab}\end{array}$ & $\begin{array}{l}\text { Lehne } \\
\text { voll } \\
\text { und ganz } \\
\quad \mathrm{ab}\end{array}$ & Mittelwert \\
\hline $\begin{array}{l}\text { Leistungsorientierte } \\
\text { Entlohnungssysteme führen zu } \\
\text { einer besseren Motivation } \\
\text { meiner Mitarbeiter. }\end{array}$ & $16,3 \%$ & $53,8 \%$ & $23,1 \%$ & $2,9 \%$ & $1 \%$ & 0,84 \\
\hline $\begin{array}{l}\text { Das Vergütungssystem ist für } \\
\text { meine Mitarbeiter leicht } \\
\text { verständlich. }\end{array}$ & $14,4 \%$ & $59,6 \%$ & $18,3 \%$ & $3,8 \%$ & & 0,88 \\
\hline $\begin{array}{l}\text { Leistungsbeurteilungen } \\
\text { erläutere ich meinen } \\
\text { Mitarbeitern ausführlich. }\end{array}$ & $6,7 \%$ & $58,7 \%$ & $18,3 \%$ & $3,8 \%$ & & 0,78 \\
\hline $\begin{array}{l}\text { Durch das leistungsorientierte } \\
\text { Entlohnungssystem fühlen sich } \\
\text { meine Mitarbeiter zu sehr } \\
\text { kontrolliert. }\end{array}$ & $1,9 \%$ & $7,7 \%$ & $24,0 \%$ & $47,1 \%$ & $9,6 \%$ & $-0,61$ \\
\hline $\begin{array}{l}\text { Leistungsorientierte } \\
\text { Entlohnung führt zu Unmut im } \\
\text { Team. }\end{array}$ & $2,9 \%$ & $8,7 \%$ & $19,2 \%$ & $47,1 \%$ & $13,5 \%$ & $-0,65$ \\
\hline $\begin{array}{l}\text { Ich achte darauf, dass die } \\
\text { Einkommensunterschiede } \\
\text { zwischen meinen Mitarbeitern } \\
\text { nicht zu hoch werden. }\end{array}$ & $6,7 \%$ & $40,4 \%$ & $24,0 \%$ & $18,3 \%$ & $2,9 \%$ & 0,32 \\
\hline $\begin{array}{l}\text { Der Kontrollaufwand } \\
\text { rechtfertigt den Erfolg des } \\
\text { Entlohnungssystems nicht. }\end{array}$ & $2,9 \%$ & $13,5 \%$ & $23,1 \%$ & $40,4 \%$ & $12,5 \%$ & $-0,50$ \\
\hline $\begin{array}{l}\text { Leistungsbeurteilungen setzen } \\
\text { klare Maßstäbe voraus. }\end{array}$ & $24,0 \%$ & 65,4 & $3,8 \%$ & & & 1,00 \\
\hline $\begin{array}{l}\text { Die Höhe der } \\
\text { Leistungszulagen wird von den } \\
\text { Mitarbeitern als ungerecht } \\
\text { empfunden. }\end{array}$ & $1,0 \%$ & $4,8 \%$ & $30,8 \%$ & $42,3 \%$ & $8,7 \%$ & $-0,60$ \\
\hline \multicolumn{7}{|c|}{$\begin{array}{l}\text { Bis zu 100\% fehlende Prozente: Keine Angaben } \\
\text { Mittelwertberechnung: hier sind die Betriebsleiter herausgerechnet, die keine Angaben gemacht haben, } \\
\text { Skalierung von }+2 \text {,stimme voll und ganz zu“ bis }-2 \text { "lehne voll und ganz ab" }\end{array}$} \\
\hline
\end{tabular}


19. In der folgenden Frage geht es um Anreizsysteme allgemein. Inwieweit treffen die folgenden Aussagen auf Sie zu? $\mathbf{n}=\mathbf{2 6 0}$

\begin{tabular}{|c|c|c|c|c|c|c|}
\hline & $\begin{array}{l}\text { Stimme } \\
\text { voll und } \\
\text { ganz zu }\end{array}$ & $\begin{array}{c}\text { Stimme } \\
\mathrm{zu}\end{array}$ & $\begin{array}{l}\text { Teils/ } \\
\text { teils }\end{array}$ & $\begin{array}{l}\text { Lehne } \\
\mathrm{ab}\end{array}$ & $\begin{array}{l}\text { Lehne } \\
\text { voll } \\
\text { und ganz } \\
\text { ab }\end{array}$ & Mittelwert \\
\hline $\begin{array}{l}\text { Das Lohnniveau in meinem } \\
\text { Betrieb ist im Vergleich zu } \\
\text { Betrieben in meiner Umgebung } \\
\text { überdurchschnittlich. }\end{array}$ & $6,5 \%$ & $27,3 \%$ & $36,9 \%$ & $21,5 \%$ & $3,5 \%$ & 0,12 \\
\hline $\begin{array}{l}\text { Gute Leistung honorieren wir } \\
\text { immer finanziell. }\end{array}$ & $1,9 \%$ & $22,3 \%$ & $46,5 \%$ & $17,7 \%$ & $3,8 \%$ & 0,01 \\
\hline $\begin{array}{l}\text { Leistungslohnsysteme brauche ich } \\
\text { in meinem Betrieb nicht, da er } \\
\text { übersichtlich strukturiert ist. }\end{array}$ & $6,9 \%$ & $32,7 \%$ & $23,5 \%$ & $26,9 \%$ & $4,6 \%$ & 0,11 \\
\hline $\begin{array}{l}\text { In der Landwirtschaft verdient man } \\
\text { sich keine ,goldene Nase“. } \\
\text { Leistungszuschläge kommen } \\
\text { meinen Mitarbeiter gerade recht. }\end{array}$ & $3,1 \%$ & $28,1 \%$ & $26,2 \%$ & $25,0 \%$ & $2,7 \%$ & 0,05 \\
\hline $\begin{array}{l}\text { Die Leistung meiner Mitarbeiter } \\
\text { spiegelt sich schon in ihrer } \\
\text { Lohngruppe wider. }\end{array}$ & $6,9 \%$ & $45,8 \%$ & $24,6 \%$ & $14,2 \%$ & $1,9 \%$ & 0,44 \\
\hline $\begin{array}{l}\text { Lob und Tadel haben einen } \\
\text { größeren Motivationseffekt als das } \\
\text { Vergütungssystem. }\end{array}$ & $6,9 \%$ & $27,7 \%$ & $48,5 \%$ & $13,1 \%$ & & 0,30 \\
\hline $\begin{array}{l}\text { Meine Mitarbeiter können wählen, } \\
\text { ob sie bei guten Leistungen mehr } \\
\text { Geld oder mehr Freizeit haben } \\
\text { wollen. }\end{array}$ & $2,7 \%$ & $8,1 \%$ & $17,3 \%$ & $47,7 \%$ & $16,5 \%$ & $-0,73$ \\
\hline $\begin{array}{l}\text { Ich versuche meine Mitarbeiter } \\
\text { durch ein attraktives Arbeitsumfeld } \\
\text { zu motivieren. }\end{array}$ & $11,5 \%$ & $66,9 \%$ & $15,8 \%$ & $1,2 \%$ & & 0,93 \\
\hline $\begin{array}{l}\text { Die Arbeit mit modernsten } \\
\text { Maschinen motiviert meine } \\
\text { Mitarbeiter. }\end{array}$ & $18,5 \%$ & $60,8 \%$ & $13,8 \%$ & $3,5 \%$ & $0,4 \%$ & 0,96 \\
\hline
\end{tabular}


20. Mit welchen Instrumenten versuchen Sie neben dem Vergütungssystem Ihre Mitarbeiter zu motivieren? $\mathbf{n}=\mathbf{2 6 0}$

\begin{tabular}{|l|c|}
\hline Betriebsfeste & $71,2 \%$ \\
\hline Gratifikationen (Geschenke, Zuwendungen, etc.) & $52,9 \%$ \\
\hline Fort- und Weiterbildungsangebote & $52,1 \%$ \\
\hline Zusätzliche Sozialleistungen (z.B. Altersvorsorge) & $54,3 \%$ \\
\hline $\begin{array}{l}\text { Individuelle Arbeitszeitgestaltung (z.B. Arbeitszeitausgleich, Abbummeln von } \\
\text { Überstunden, Teilzeit) }\end{array}$ & $65,4 \%$ \\
\hline Moderne Maschinen & $71,2 \%$ \\
\hline Gutes Betriebsklima & $84,0 \%$ \\
\hline Sicherer Arbeitsplatz & $78,6 \%$ \\
\hline $\begin{array}{l}\text { Sonstiges: Deputate, Familienanschluss, Unterstützung im privaten Umfeld, Einbeziehung der } \\
\text { Familie, Mitbewirtschaftung mitarbeitereigener Flächen, Unterstützung mit Maschinen, Einbeziehung } \\
\text { beim Maschinenkauf/Investitionsentscheidungen, monatliches Gespräch über aktuelle Probleme und } \\
\text { Ziele im Betrieb, im Gespräch bleiben }\end{array}$ \\
\hline
\end{tabular}

\section{Wie ist die Arbeitszeit in Ihrem Betrieb geregelt? Bitte kreuzen Sie zutreffende} Antworten an. $\mathbf{n}=\mathbf{2 6 0}$

\begin{tabular}{|l|c|c|c|}
\hline & ja & nein & $\begin{array}{c}\text { Keine } \\
\text { Angabe }\end{array}$ \\
\hline In meinem Betrieb gibt es Teilzeitstellen. & $45,8 \%$ & $46,2 \%$ & $8,1 \%$ \\
\hline $\begin{array}{l}\text { Die Lage und Länge der Arbeitszeit meiner Mitarbeiter variieren } \\
\text { je nach betrieblichen Erfordernissen. }\end{array}$ & $95,8 \%$ & $1,5 \%$ & $2,7 \%$ \\
\hline $\begin{array}{l}\text { Lage und Länge der Arbeitszeit richten sich nach den } \\
\text { persönlichen Wünschen meiner Mitarbeiter. }\end{array}$ & $16,9 \%$ & $71,2 \%$ & $11,9 \%$ \\
\hline $\begin{array}{l}\text { Beginn und Ende der täglichen Arbeitszeit können meine } \\
\text { Mitarbeiter (in bestimmten Grenzen) selbst bestimmen. }\end{array}$ & $32,3 \%$ & $61,9 \%$ & $5,8 \%$ \\
\hline $\begin{array}{l}\text { Ich habe mit meinen Mitarbeitern vereinbart, dass ich sie bei } \\
\text { Bedarf kurzfristig anrufen kann. }\end{array}$ & $83,7 \%$ & $10,5 \%$ & $5,8 \%$ \\
\hline In meinem Betrieb werden Arbeitzeitkonten geführt. & $54,6 \%$ & $38,1 \%$ & $7,3 \%$ \\
\hline $\begin{array}{l}\text { Mehrarbeit, z.B. während der Ernte, können meine Mitarbeiter in } \\
\text { ruhigeren Zeiten abbummeln. }\end{array}$ & $81,2 \%$ & $13,8 \%$ & $5,0 \%$ \\
\hline
\end{tabular}


22. Die folgenden Statements beziehen sich auf ihre Arbeitszeitgestaltung. Bitte geben Sie Ihre Zustimmung bzw. Ablehnung an. n=141

Falls Sie keine flexiblen Arbeitszeiten haben, dann geht es mit Frage 23 weiter.

\begin{tabular}{|l|c|c|c|c|c|c|}
\hline & $\begin{array}{c}\text { Stimme } \\
\text { voll und } \\
\text { ganz zu }\end{array}$ & $\begin{array}{c}\text { Stimme } \\
\text { zu }\end{array}$ & $\begin{array}{c}\text { Teils/ } \\
\text { teils }\end{array}$ & $\begin{array}{c}\text { Lehne } \\
\text { ab }\end{array}$ & $\begin{array}{c}\text { Lehne } \\
\text { voll und } \\
\text { ganz ab }\end{array}$ & Mittelwert \\
\hline $\begin{array}{l}\text { Durch das realisierte } \\
\text { Arbeitszeitmodell spare ich } \\
\text { Überstundenzuschläge. }\end{array}$ & $22,1 \%$ & $44,3 \%$ & $12,1 \%$ & $12,9 \%$ & $2,1 \%$ & 0,76 \\
\hline $\begin{array}{l}\text { Meine Mitarbeiter sind mit unserem } \\
\text { Arbeitszeitmodell zufrieden. }\end{array}$ & $13,6 \%$ & $64,3 \%$ & $18,6 \%$ & $2,1 \%$ & & 0,91 \\
\hline $\begin{array}{l}\text { Dank der Flexibilisierung der } \\
\text { Arbeitszeit fällt es mir leichter, } \\
\text { neue Mitarbeiter zu finden. }\end{array}$ & $1,4 \%$ & $14,3 \%$ & $42,9 \%$ & $23,6 \%$ & $5,7 \%$ & $-0,20$ \\
\hline $\begin{array}{l}\text { Die Arbeitszeitflexibilisierung hat } \\
\text { zusätzliche Kosten (Verwaltung, } \\
\text { Zeiterfassung) verursacht. }\end{array}$ & $1,4 \%$ & $13,6 \%$ & $20,0 \%$ & $47,9 \%$ & $7,9 \%$ & $-0,52$ \\
\hline $\begin{array}{l}\text { Die Arbeitszeitflexibilisierung } \\
\text { erhöht die Motivation meiner } \\
\text { Mitarbeiter. }\end{array}$ & $8,6 \%$ & $39,3 \%$ & $34,3 \%$ & $11,4 \%$ & & 0,48 \\
\hline $\begin{array}{l}\text { Insgesamt sparen wir durch die } \\
\text { Arbeitszeitflexibilisierung Geld. }\end{array}$ & $16,4 \%$ & $46,4 \%$ & $22,1 \%$ & $6,4 \%$ & $0,7 \%$ & 0,78 \\
\hline $\begin{array}{l}\text { Seit der Arbeitszeitflexibilisierung } \\
\text { sind meine Mitarbeiter seltener } \\
\text { krank. }\end{array}$ & $2,1 \%$ & $17,9 \%$ & $35,7 \%$ & $25,0 \%$ & $5,0 \%$ & $-0,15$ \\
\hline $\begin{array}{l}\text { Bis zu 100\% fehlende Prozente: Keine Angaben } \\
\text { Mittelwertberechnung: hier sind die Betriebsleiter herausgerechnet, die keine Angaben } \\
\text { haben, Skalierung von + , stimme voll und ganz zu“ bis -2, lehne voll und ganz ab“ }\end{array}$ \\
\hline
\end{tabular}
23.
Aus
welchem
Bundesland
kommen
Sie?

\section{Welche Rechtsform hat Ihr Betrieb?}

\begin{tabular}{|l|c|c|}
\hline Rechtsform: & Nennungen: & Prozent: \\
\hline Einzelunternehmen & $\mathrm{n}=73$ & $28,1 \%$ \\
\hline GbR & $\mathrm{n}=58$ & $22,3 \%$ \\
\hline $\mathrm{GmbH}$ & $\mathrm{n}=45$ & $17,3 \%$ \\
\hline OHG & $\mathrm{n}=0$ & $0 \%$ \\
\hline $\mathrm{eG}$ & $\mathrm{n}=56$ & $21,5 \%$ \\
\hline KG & $\mathrm{n}=17$ & $6,5 \%$ \\
\hline Sonstige, und zwar...... & $\mathrm{n}=11$ & $4,2 \%$ \\
\hline
\end{tabular}




\section{Zu welcher Betriebsform gehört Ihr Betrieb?}

(Richtet sich danach, welcher Betriebszweig 2/3 des Gesamt-Standarddeckungsbeitrages erbringt)

\begin{tabular}{|l|c|c|}
\hline Ackerbau & $\mathrm{n}=79$ & $30,5 \%$ \\
\hline Futterbau (Milchvieh, Rinder......) & $\mathrm{n}=45$ & $17,4 \%$ \\
\hline Veredelung (Schwein, Geflügel.....) & $\mathrm{n}=20$ & $7,7 \%$ \\
\hline Verbund & $\mathrm{n}=64$ & $24,7 \%$ \\
\hline Sonstige & $\mathrm{n}=51$ & $19,7 \%$ \\
\hline
\end{tabular}

26. Welcher Wirtschaftsweise ist Ihr Betrieb zuzuordnen? $\mathbf{n = 2 5 9}$

88,0\% konventioneller Betrieb

11,6\% ökologischer Betrieb (Teilnahme am Kontrollverfahren nach der EG-ÖkoVerordnung)

27. Welche Position üben Sie im Betrieb aus? $\mathbf{n}=\mathbf{2 6 0}$

94,2\% Betriebsleiter/in, Geschäftsführer/in

$3,1 \% \quad$ Hofnachfolger/-in

0,4\% Leitung Milchproduktion

2,3\% Leitung Schweineproduktion

28. Haben Sie sich schon Gedanken zur Hofnachfolge gemacht? $\mathbf{n}=\mathbf{2 5 1}$

$29,6 \%$ Ja, die Hofnachfolge steht schon fest.

13,8\% Ja, aber noch kein Ergebnis erzielt.

0,8\% Der Betrieb läuft aus.

50,4\% Nein, die Frage steht noch nicht an.

$3,1 \% \quad$ Sonstige 
29. Welche berufliche Ausbildung haben Sie abgeschlossen (höchster Abschluss)? $\mathrm{n}=\mathbf{2 5 6}$

2,7\% Landwirtschaftliche Lehre

$3,5 \% \quad$ Staatlich geprüfter Wirtschafter

8,8\% Fachschule/ staatl. geprüfter Agrarbetriebswirt

$12,7 \%$ Meister

68,8\% Landwirtschaftliches Studium (FH / Uni)

$3,1 \% \quad$ Keine landwirtschaftliche Ausbildung

$0,4 \% \quad$ keine Angabe

30. Welchem Geburtsjahrgang gehören Sie an?

31. Seit wann bewirtschaften Sie den Betrieb?

32. Welches Geschlecht haben Sie?

$91,1 \% \mathrm{~m}$

$8,5 \% \quad$ w

\section{Herzlichen Dank für Ihre Unterstützung!}




\section{TEIL II}

\section{Ergebnisse der Mitarbeiterbefragung}




\section{Ausgewählte Ergebnisse der Mitarbeiterbefragung in Kürze:}

- 124 Arbeitnehmer aus landwirtschaftlichen Betrieben in Niedersachsen

- Gut ausgebildete Fachkräfte, vorwiegend im Ackerbau tätig

- 93,5 \% der befragten Mitarbeiter schätzen die Tätigkeit in der Landwirtschaft

Statistische Tests ergaben, dass den Mitarbeiter die Arbeit besonders dann Freude macht, wenn...

- die Betriebsleiter die Mitarbeiter in betriebliche Entscheidungen einbinden,

- die Arbeitsleistung vom Chef auch gewürdigt wird,

- die vom Chef gesetzten Ziele auch erreichbar sind.

Negativ auf die Motivation wirken:

- Die Wahrnehmung von ungerechter Bezahlung im Vergleich zu anderen Mitarbeitern im Betrieb und in vergleichbaren Betrieben.

> Das Ungerechtigkeitsempfinden kann gesenkt werden, indem deutlich gemacht wird, wie unterschiedliche Löhne zustande kommen. Hier besteht aus Sicht der Mitarbeiter noch Verbesserungsbedarf.

Die wichtigsten Anreizsysteme aus Perspektive der Mitarbeiter sind:

- Ein sicherer Arbeitsplatz, ein gutes Betriebsklima, die Lohngestaltung und -höhe, der Arbeitsinhalt, moderne Maschinen

- Für ein Drittel der Mitarbeiter sind immaterielle Anreize wichtiger als die Bezahlung.

- Aufgrund des niedrigen Lohnniveaus schätzen die Mitarbeiter aber finanzielle Anreize.

Erfahrungen der Mitarbeiter mit variablen Entlohnungssystemen:

- Die Mitarbeiter, die Erfahrung mit variablen Vergütungsformen gesammelt haben, sind überwiegend damit zufrieden.

- Eine Ergebnisdiskussion mit den Mitarbeitern machte deutlich, dass die Mehrzahl der von den Betriebsleitern verwendeten Leistungsindikatoren von den Mitarbeitern als zu subjektiv und störanfällig eingeschätzt wird.

Akzeptable Leistungsindikatoren aus Sicht der Mitarbeiter sind:

- Im Ackerbau: positive Entwicklung der Hektarerträge im Vergleich zum mehrjährigen Betriebsdurchschnitt

- In der Milchproduktion: positive Entwicklung der Milchleistung, Milchqualität

- In der Sauenhaltung: aufgezogene Ferkel pro Sau und Jahr 


\section{Häufigkeitsauszählungen und Mittelwerte des Mitarbeiterfragebogens}

1. Wie beurteilen Sie die folgenden Aussagen zu ihrer Tätigkeit? Bitte geben Sie den Grad Ihrer Zustimmung bzw. Ablehnung an!* n=119-121

\begin{tabular}{|c|c|c|c|c|c|c|}
\hline & $\begin{array}{l}\text { Stimme } \\
\text { voll und } \\
\text { ganz zu }\end{array}$ & $\begin{array}{l}\text { Stimme } \\
\mathrm{zu}\end{array}$ & $\begin{array}{l}\text { Teils/ } \\
\text { teils }\end{array}$ & $\begin{array}{l}\text { Lehne } \\
\mathrm{ab}\end{array}$ & $\begin{array}{l}\text { Lehne } \\
\text { voll } \\
\text { und ganz } \\
\text { ab }\end{array}$ & $\begin{array}{c}\text { Mittel- } \\
\text { wert }\end{array}$ \\
\hline $\begin{array}{l}\text { Mein Chef spricht regelmäßig } \\
\text { mit mir über die Ziele des } \\
\text { Unternehmens. }\end{array}$ & $16,3 \%$ & $40,7 \%$ & $37,4 \%$ & $4,9 \%$ & $0 \%$ & 0,76 \\
\hline $\begin{array}{l}\text { Ich kann die Ziele des } \\
\text { Unternehmens klar benennen. }\end{array}$ & $14,6 \%$ & $42,3 \%$ & $39,8 \%$ & $1,6 \%$ & $0 \%$ & 0,85 \\
\hline $\begin{array}{l}\text { Ich weiß, wie ich zur } \\
\text { Realisierung der } \\
\text { Unternehmensziele beitragen } \\
\text { kann. }\end{array}$ & $16,3 \%$ & $58,3 \%$ & $19,5 \%$ & $3,3 \%$ & $0 \%$ & 1,10 \\
\hline $\begin{array}{l}\text { Chef setzt Ziele so, dass ich } \\
\text { sie auch erreichen kann. }\end{array}$ & $17,9 \%$ & $49,6 \%$ & $23,6 \%$ & $4,9 \%$ & $0,8 \%$ & 1,08 \\
\hline $\begin{array}{l}\text { Ich habe die Hilfsmittel und } \\
\text { Technik, die ich benötige, um } \\
\text { gute Arbeit zu leisten. }\end{array}$ & $28,5 \%$ & $44,7 \%$ & $22,0 \%$ & $1,6 \%$ & $0,8 \%$ & 1,20 \\
\hline $\begin{array}{l}\text { Die Arbeit in der } \\
\text { Landwirtschaft macht mir } \\
\text { Spaß. }\end{array}$ & $71,5 \%$ & $22,0 \%$ & $4,9 \%$ & $0 \%$ & $0,8 \%$ & 1,71 \\
\hline $\begin{array}{l}\text { Mein Chef bindet mich bei } \\
\text { betrieblichen Entscheidungen } \\
\text { ein. }\end{array}$ & $13,8 \%$ & $27,6 \%$ & $46,3 \%$ & $7,3 \%$ & $4,1 \%$ & 0,47 \\
\hline $\begin{array}{l}\text { Mein Arbeitseinsatz wird von } \\
\text { meinem Chef auch } \\
\text { entsprechend gewürdigt. }\end{array}$ & $17,9 \%$ & $54,5 \%$ & $23,6 \%$ & $3,3 \%$ & $0 \%$ & 0,94 \\
\hline
\end{tabular}

2. Wie wird derzeit Ihr Gehalt/Lohn bemessen? Mehrfachantworten sind möglich, $n=123$

\begin{tabular}{|l|c|}
\hline Fester Stundenlohn/Gehalt & $83,7 \%$ \\
\hline Zuschläge für Überstunden & $57,7 \%$ \\
\hline Zuschläge für Sonn- und Feiertagsarbeit & $50,4 \%$ \\
\hline Zulagen für Arbeitserschwernis (z.B. Pflanzenschutzmaßnahmen) & $26,8 \%$ \\
\hline Ich erhalte Zulagen für bestimmte Leistungen. & $19,5 \%$ \\
\hline Erfolgsbeteiligungen & $14,6 \%$ \\
\hline $\begin{array}{l}\text { Sonstige: Belegschaftsaktien, Erbfolge, stundengenaue Abrechnung des Lohns, Weihnachtsgeld, } \\
\text { Überstunden abfeiern, Erholungsurlaub }\end{array}$ \\
\hline
\end{tabular}


3. Haben Sie bereits Erfahrungen mit leistungsorientierter Bezahlung gemacht? $\mathrm{n}=123$

\begin{tabular}{|l|c|}
\hline Ja & $33,3 \%$ \\
\hline Nein & $64,2 \%$ \\
\hline Keine Angabe & $2,4 \%$ \\
\hline
\end{tabular}

3a) Wenn ja, wie zufrieden waren Sie mit dieser Form der Bezahlung? $n=41$

$\begin{array}{ccccc}\text { sehr zufrieden } & \text { eher zufrieden } & \text { teils/teils } & \text { eher unzufrieden } & \text { sehr unzufrieden } \\ 39 \% & 31,7 \% & 26,8 \% & 2,4 \% & 0 \%\end{array}$

4. In der nächsten Frage geht es um Ihre Bezahlung. Bitte geben Sie wieder an inwieweit Sie den folgenden Aussagen zustimmen?* n=75-109

\begin{tabular}{|l|c|c|c|c|c|c|c|}
\hline & $\begin{array}{c}\text { Stimme } \\
\text { voll und } \\
\text { ganz zu }\end{array}$ & $\begin{array}{c}\text { Stimme } \\
\text { zu }\end{array}$ & $\begin{array}{c}\text { Teils/ } \\
\text { teils }\end{array}$ & $\begin{array}{c}\text { Lehne } \\
\text { ab }\end{array}$ & $\begin{array}{c}\text { Lehne voll } \\
\text { und ganz ab }\end{array}$ & $\begin{array}{c}\text { Keine } \\
\text { Aussage } \\
\text { möglich }\end{array}$ & $\begin{array}{c}\text { Mittel- } \\
\text { wert }\end{array}$ \\
\hline $\begin{array}{l}\text { Zulagen, die ich für } \\
\text { meine Leistung } \\
\text { erhalte, verbessern } \\
\text { meine Motivation. }\end{array}$ & $26,8 \%$ & $34,1 \%$ & $16,3 \%$ & $2,4 \%$ & $0 \%$ & $14,6 \%$ & 1,07 \\
\hline $\begin{array}{l}\text { Ich verstehe, wie } \\
\text { Löhne und Gehälter } \\
\text { in unserem Betrieb } \\
\text { festgelegt werden. }\end{array}$ & $15,4 \%$ & $37,4 \%$ & $26,8 \%$ & $5,7 \%$ & $1,6 \%$ & $9,8 \%$ & 0,68 \\
\hline $\begin{array}{l}\text { Mein Chef erläutert } \\
\text { uns ausführlich, wie } \\
\begin{array}{l}\text { Leistungszulagen } \\
\text { zustande kommen. }\end{array}\end{array}$ & $6,5 \%$ & $22,0 \%$ & $26,0 \%$ & $12,2 \%$ & $4,1 \%$ & $24,4 \%$ & 0,21 \\
\hline $\begin{array}{l}\text { Ich fühle ich mich } \\
\text { bei der Bezahlung } \\
\text { gegenüber meinen } \\
\text { Kollegen im Betrieb } \\
\text { ungerecht behandelt. }\end{array}$ & $0,8 \%$ & $8,1 \%$ & $19,5 \%$ & $16,3 \%$ & $16,3 \%$ & $30,1 \%$ & $-0,64$ \\
\hline $\begin{array}{l}\text { In unserem Betrieb } \\
\text { bekomme ich für } \\
\text { meine Tätigkeit } \\
\text { weniger Geld als in } \\
\text { vergleichbaren } \\
\text { Betrieben. }\end{array}$ & $4,1 \%$ & $8,1 \%$ & $17,9 \%$ & $13,0 \%$ & $20,3 \%$ & $32,5 \%$ & $-0,59$ \\
\hline $\begin{array}{l}\text { Lob und Tadel } \\
\text { motivieren mich } \\
\text { mehr als die } \\
\text { Bezahlung. }\end{array}$ & $12,2 \%$ & $21,1 \%$ & $37,4 \%$ & 13,0 & $4,9 \%$ & $5,7 \%$ & 0,26 \\
\hline $\begin{array}{l}\text { Auf Zuschläge und } \\
\text { Prämien bin ich } \\
\text { finanziell } \\
\text { angewiesen. }\end{array}$ & $2,4 \%$ & $15,4 \%$ & $33,3 \%$ & $15,4 \%$ & $11,4 \%$ & $17,1 \%$ & $-0,23$ \\
\hline Skalierung von +2 ,stimme voll und ganz zu“ bis -2, ,lehne voll und ganz ab & & & & & \\
\hline
\end{tabular}


5. Wie wichtig sind die nachfolgenden Dinge für Ihre Motivation? Bitte geben Sie eine Rangfolge von 1-10 an (1=wichtigstes Instrument, 10=unwichtigstes Instrument)

\begin{tabular}{|l|c|c|}
\hline Motivation durch: & $\mathbf{n}$ & Wichtigkeit*: \\
\hline Betriebsfeste & 105 & $4,1 \%$ \\
\hline Lohngestaltung (Höhe des Gehalts, Art der Bezahlung) & 106 & $29,3 \%$ \\
\hline Arbeitsinhalt & 105 & $24,4 \%$ \\
\hline Fort- und Weiterbildungsangebote & 106 & $12,2 \%$ \\
\hline Zusätzliche Sozialleistungen (z.B. Altersvorsorge) & 107 & $26,8 \%$ \\
\hline $\begin{array}{l}\text { Individuelle Arbeitszeitgestaltung (z.B. Arbeitszeitausgleich, } \\
\text { Abbummeln von Überstunden, Teilzeit) }\end{array}$ & 103 & $15,4 \%$ \\
\hline Moderne Maschinen & 105 & $19,5 \%$ \\
\hline Gutes Betriebsklima & 106 & $36,6 \%$ \\
\hline Sicherer Arbeitsplatz & 107 & $47,2 \%$ \\
\hline Sonstiges................. & \\
\hline *Gibt an, wie viel Prozent der Antwortenden das Anreizsystem als wichtigsten Motivator nennen \\
\hline
\end{tabular}

\section{Inwieweit treffen die folgenden Aussagen auf Sie persönlich zu?* $n=115-118$}

\begin{tabular}{|c|c|c|c|c|c|c|}
\hline & $\begin{array}{l}\text { Stimme voll } \\
\text { und ganz zu }\end{array}$ & $\begin{array}{l}\text { Stimme } \\
\mathrm{zu}\end{array}$ & $\begin{array}{l}\text { Teils/ } \\
\text { teils }\end{array}$ & $\begin{array}{l}\text { Lehne } \\
\mathrm{ab}\end{array}$ & $\begin{array}{l}\text { Lehne voll } \\
\text { und ganz ab }\end{array}$ & Mittelwert \\
\hline $\begin{array}{l}\text { Risiko einzugehen kann } \\
\text { Spaß machen. }\end{array}$ & $5,7 \%$ & $26,8 \%$ & $40,7 \%$ & $16,3 \%$ & $4,1 \%$ & 0,67 \\
\hline $\begin{array}{l}\text { Ich tue manchmal Dinge } \\
\text { aus Spaß, von denen ich } \\
\text { weiß, dass sie gefährlich } \\
\text { sind. }\end{array}$ & $4,1 \%$ & $17,9 \%$ & $23,6 \%$ & $34,1 \%$ & $14,6 \%$ & 0,14 \\
\hline $\begin{array}{l}\text { Ich gehe lieber auf } \\
\text { Nummer sicher, als } \\
\text { hinter etwas zu bereuen. }\end{array}$ & $22 \%$ & $41,5 \%$ & $28,5 \%$ & $4,1 \%$ & $0 \%$ & 1,18 \\
\hline $\begin{array}{l}\text { Ich versuche Risiken zu } \\
\text { vermeiden, auch wenn } \\
\text { ich dadurch Chancen } \\
\text { verpasse. }\end{array}$ & $13,0 \%$ & $28,5 \%$ & $42,3 \%$ & $9,8 \%$ & $0,8 \%$ & 0,94 \\
\hline $\begin{array}{l}\text { Ich mag es gerne, wenn } \\
\text { alles so bleibt wie es ist. }\end{array}$ & $8,1 \%$ & $11,4 \%$ & $43,1 \%$ & $22,8 \%$ & $8,1 \%$ & 0,42 \\
\hline
\end{tabular}


7. Aus welchem Bundesland kommen Sie?

8. Welches ist der wichtigste Betriebszweig in ihrem Betrieb?

\begin{tabular}{|l|c|}
\hline Ackerbau & $69,7 \%$ \\
\hline Futterbau (Milchvieh, Rinder......) & $0 \%$ \\
\hline Veredelung (Schwein, Geflügel......) & $0,8 \%$ \\
\hline Dauerkulturen (Weinbau, Obstbau, Spargel...) & $1,7 \%$ \\
\hline Verbund & $16,8 \%$ \\
\hline Sonstige & $10,9 \%$ \\
\hline
\end{tabular}

9. Wie groß ist der Betrieb auf dem Sie arbeiten? Mittelwerte

332,38 ha Ackerbau

42,40 ha Grünland

10. Wie viel Vieh wird auf dem Betrieb gehalten?

85,11 Milchkühe

775 Mastschweine (Plätze)

101,25 Sauen

Sonstiges

(Tierart eintragen)

11. Wie viele Familienangehörige und Fremdarbeitskräfte arbeiten auf Ihrem Betrieb? (Bitte die Anzahl an Personen angeben!)

\begin{tabular}{|l|c|c|c|c|c|}
\hline $\begin{array}{l}\text { Art der Arbeitskräfte } \\
\text { (AK) }\end{array}$ & Nennungen & Minimum & Maximum & Mittelwert & Median \\
\hline $\begin{array}{l}\text { Familienarbeitskräfte } \\
\text { (Anzahl Personen) }\end{array}$ & $\mathrm{n}=62$ & 1 & 18 & 2,18 & 1 \\
\hline $\begin{array}{l}\text { Fremdarbeitskräfte } \\
\text { (Anzahl Personen) }\end{array}$ & $\mathrm{n}=97$ & 1 & 1200 & 54,26 & 2 \\
\hline Davon Auszubildende & $\mathrm{n}=33$ & 1 & 77 & 14,76 & 1 \\
\hline
\end{tabular}


12. Welche Position üben Sie im Betrieb aus?

6,5\% Betriebsleiter/in, Geschäftsführer/in

81,3\% Mitarbeiter Ackerbau

3,3\% Mitarbeiter Milchproduktion

5,7\% Mitarbeiter Schweineproduktion

13. Welche berufliche Ausbildung haben Sie abgeschlossen (höchster Abschluss)? n=122

$33,6 \%$ Landwirtschaftliche Lehre

$13,1 \%$ Staatlich geprüfter Wirtschafter

11,5\% Fachschule/ staatl. geprüfter Agrarbetriebswirt

$13,1 \%$ Meister

$1,6 \%$ Landwirtschaftliches Studium (FH / Uni)

9,8\% Keine landwirtschaftliche Ausbildung

$17,1 \%$ Sonstige

14. Welchem Geburtsjahrgang gehören Sie an?

15. Seit wie vielen Jahren arbeiten Sie bei ihrem jetzigen Arbeitsgeber?

weniger als 2 Jahre: $\quad 3,3 \%$

2-5 Jahre: $\quad 12,2 \%$

6-10 Jahre: $\quad 13,8 \%$

mehr als 10 Jahre: $\quad 70,7 \%$

16. Welches Geschlecht haben Sie?

$100 \% \mathrm{~m}$

$0 \% \quad \mathrm{w}$

\section{Herzlichen Dank für Ihre Unterstützung!}




\section{LEBENSLAUF}

\section{PERSÖNLIChe DATEN}

Name: $\quad$ Zazie von Davier

Adresse: $\quad$ Gutshof 2

$\begin{array}{ll} & 39356 \text { Seggerde } \\ \text { e-mail: } & \text { zdavier@gwdg.de }\end{array}$

geboren am: $\quad$ 02. November 1974 in Berlin

\section{AUSBILDUNG}

$08 / 87-06 / 94$

Schiller-Gymnasium-Berlin, Abitur, Note 1,8

07/94 - 07/96

Ausbildung zur Landwirtin, Note der

Abschlussprüfung 1,3

$10 / 96-07 / 98$

Georg-August-Universität Göttingen

- Grundstudium der Agrarwissenschaften, Vordiplom, Note 1,7

09/98 - 04/99

Ecole Nationale Supérieure Agronomique de Rennes,

Frankreich

- Auslandssemester als Stipendiatin des ERASMUS-Programms

04/99 - 07/01 Georg-August-Universität Göttingen

- Hauptstudium der Agrarwissenschaften, Fachrichtung Agribusiness, Abschluss als Master of Science (entspricht dem Diplom-Agraringenieur), Note 1,5

- Masterarbeit bei Herrn Prof. Dr. M. Köhne, Thema: Englische Bewirtschaftungsmodelle und ihre Übertragbarkeit auf Deutschland, Note 1,7

Seit 01/04 Georg-August-Universität Göttingen

- Promotion im Arbeitsbereich angewandte landwirtschaftliche Betriebslehre, Thema: „Leistungsorientierte Entlohnung in der Landwirtschaft: eine empirische Analyse“"

- Leitung des Tutoriums zur Lehrveranstaltung Einführung in die landwirtschaftliche Marktlehre, 150 Teilnehmer im SS 2005 


\section{PrakTiKa Und Bisherige TÄTigkeiten}

$04 / 97$

Landberatung Gifhorn-Wolfsburg

- Einzelbetriebliche Beratung

(Betriebswirtschaftliche

Beratung, Rinderspezialberatung)

- Anfertigung einer vergleichenden Studie über die Rindfleischproduktion in Deutschland und Frankreich

08/99 - 10/99 Strachan Farms Ltd. England

$09 / 00-10 / 00$

- Erntehelferin, zeitweilige Vertretung des Betriebsleiters auf einem 800 ha Marktfrucht-/ Futterbaubetrieb mit 200 Milchkühen und 250 Fleischrindern

10/00 - 03/01 Georg-August-Universität Göttingen

- Leitung des Tutoriums zur Lehrveranstaltung Mikroökonomie, 35 Teilnehmer

08/01 - 10/01 University of Cambridge

- Freie wissenschaftliche Mitarbeiterin für ein Forschungsprojekt

11/01 -04/02 Betriebswirtschaftliches Büro Göttingen

- Durchführung von Betriebsanalysen und Betriebsvergleichen

- Investitionsplanungen und Kooperationsberatung

05/02 - 12/03 Bundesforschungsanstalt für Landwirtschaft

- Wissenschaftliche Mitarbeitern beim Aufbau des International Farm Comparison Network (IFCN) für den Produktbereich Rindfleisch

- Länderverantwortliche im Netzwerk für Frankreich, Australien, Namibia, Großbritannien und Österreich

\section{$\underline{\text { SPRACHEN }}$}

- Englisch (sehr gut)

- Französisch (fließend)

- Portugiesisch (Grundkenntnisse)

\section{EHRENÄMTER}

- Ehrenamtliche Übersetzerin (Englisch-Deutsch) für das Kinderhilfswerk Plan International Deutschland e.V. 